\title{
Physics, Astrophysics and Cosmology with Gravitational Waves
}

\author{
B.S. Sathyaprakash \\ School of Physics and Astronomy, Cardiff University, \\ Cardiff, U.K. \\ email: B.Sathyaprakash@astro.cf.ac.uk \\ Bernard F. Schutz \\ School of Physics and Astronomy, Cardiff University, \\ Cardiff, U.K. \\ and \\ Max Planck Institute for Gravitational Physics \\ (Albert Einstein Institute) \\ Potsdam-Golm, Germany \\ email: Bernard.Schutz@aei.mpg.de

\section{Living Reviews in Relativity} \\ ISSN 1433-8351 \\ Accepted on 29 January 2009 \\ Published on 4 March 2009
}

\begin{abstract}
Gravitational wave detectors are already operating at interesting sensitivity levels, and they have an upgrade path that should result in secure detections by 2014 . We review the physics of gravitational waves, how they interact with detectors (bars and interferometers), and how these detectors operate. We study the most likely sources of gravitational waves and review the data analysis methods that are used to extract their signals from detector noise. Then we consider the consequences of gravitational wave detections and observations for physics, astrophysics, and cosmology.
\end{abstract}

This review is licensed under a Creative Commons Attribution-Non-Commercial-NoDerivs 3.0 Germany License. http://creativecommons.org/licenses/by-nc-nd/3.0/de/ 


\section{Imprint / Terms of Use}

Living Reviews in Relativity is a peer reviewed open access journal published by the Max Planck Institute for Gravitational Physics, Am Mühlenberg 1, 14476 Potsdam, Germany. ISSN 1433-8351.

This review is licensed under a Creative Commons Attribution-Non-Commercial-NoDerivs 3.0

Germany License: http://creativecommons.org/licenses/by-nc-nd/3.0/de/

Because a Living Reviews article can evolve over time, we recommend to cite the article as follows:

B.S. Sathyaprakash and Bernard F. Schutz,

"Physics, Astrophysics and Cosmology with Gravitational Waves",

Living Rev. Relativity, 12, (2009), 2. [Online Article]: cited [<date $>$ ],

http://www.livingreviews.org/lrr-2009-2

The date given as $<$ date $>$ then uniquely identifies the version of the article you are referring to.

\section{Article Revisions}

Living Reviews supports two different ways to keep its articles up-to-date:

Fast-track revision A fast-track revision provides the author with the opportunity to add short notices of current research results, trends and developments, or important publications to the article. A fast-track revision is refereed by the responsible subject editor. If an article has undergone a fast-track revision, a summary of changes will be listed here.

Major update A major update will include substantial changes and additions and is subject to full external refereeing. It is published with a new publication number.

For detailed documentation of an article's evolution, please refer always to the history document of the article's online version at http://www.livingreviews.org/lrr-2009-2. 


\section{Contents}

1 A New Window onto the Universe $\quad 7$

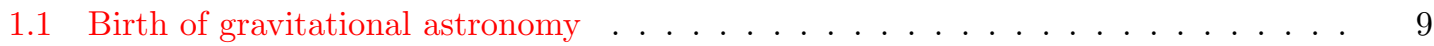

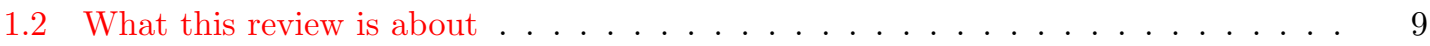

2 Gravitational Wave Observables $r$

2.1 Gravitational field vs gravitational waves . . . . . . . . . . . . . . . 11

2.2 Gravitational wave polarizations . . . . . . . . . . . . . . . . . . 12

2.3 Direction to a source . . . . . . . . . . . . . . . . . . . . . . . 12

2.4 Amplitude of gravitational waves - the quadrupole approximation . . . . . . . . 13

2.4.1 Wave amplitudes and polarization in TT-gauge . . . . . . . . . . . 13

2.4.2 Simple estimates ... . . . . . . . . . . . . . . . . . . . . . . . . . . . . . . . . 14

2.5 Frequency of gravitational waves . . . . . . . . . . . . . . . . . . . . . . . . . . . . . . . . . . . . .

2.6 Luminosity in gravitational waves . . . . . . . . . . . . . . . . . . . 16

3 Sources of Gravitational Waves $\quad 18$

3.1 Man-made sources . . . . . . . . . . . . . . . . . . . . . . 18

3.2 Gravitational wave bursts from gravitational collapse . . . . . . . . . . . . . . . 18



3.4 Radiation from a binary star system . . . . . . . . . . . . . . . . . . 21

3.4.1 Radiation from a binary system and its backreaction . . . . . . . . . . . 21

3.4.2 Chirping binaries as standard sirens . . . . . . . . . . . . . . . . . 22

3.4.3 Binary pulsar tests of gravitational radiation theory . . . . . . . . . . 23

3.4.4 White-dwarf binaries . . . . . . . . . . . . . . . . . . 23

3.4.5 Supermassive black hole binaries . . . . . . . . . . . . . . . . . . 24

3.4.6 Extreme and intermediate mass-ratio inspiral sources . . . . . . . . . . 24

3.5 Quasi-normal modes of a black hole . . . . . . . . . . . . . . . . . . . . . . . . . . . . . . . . . .



4 Gravitational Wave Detectors and Their Sensitivity 29

4.1 Principles of the operation of resonant mass detectors . . . . . . . . . . . . . . . . 29

4.2 Principles of the operation of beam detectors . . . . . . . . . . . . . . . . . 31

4.2.1 The response of a ground-based interferometer . . . . . . . . . . . . . . . 32

4.3 Practical issues of ground-based interferometers . . . . . . . . . . . . . . . 36

4.3.1 Interferometers around the globe . . . . . . . . . . . . . . . . . . . . . . . . . . .

4.3.2 Very-high-frequency detectors . . . . . . . . . . . . . . . . . 40

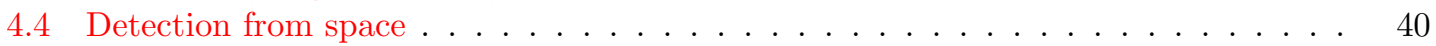

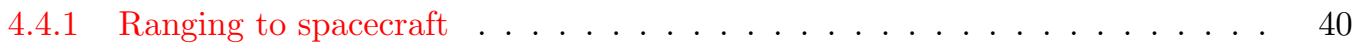

4.4 .2 Pulsar timing . . . . . . . . . . . . . . . . . . . . . . . . . . . . . .

4.4 .3 Space interferometry . . . . . . . . . . . . . . . . 41

4.5 Characterizing the sensitivity of a gravitational wave antenna . . . . . . . . . . . 42

4.5.1 Noise power spectral density in interferometers . . . . . . . . . . . . 43

4.5.2 Sensitivity of interferometers in units of energy flux . . . . . . . . . . . . 45

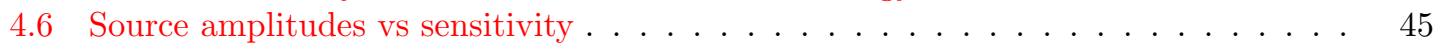

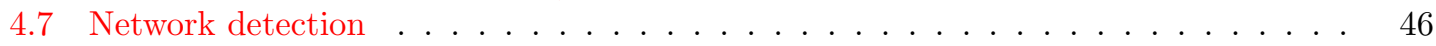

4.7.1 Coherent vs coincidence analysis . . . . . . . . . . . . . . . . 47

4.7 .2 Null stream veto . . . . . . . . . . . . . . . . . . . . . . . . . . . . . . . . 48

4.7.3 Detection of stochastic signals by cross-correlation . . . . . . . . . . . 48

4.8 False alarms, detection threshold and coincident observation . . . . . . . . . . . 49 
5 Data Analysis $\quad 51$

5.1 Matched filtering and optimal signal-to-noise ratio . . . . . . . . . . . . . . . 52

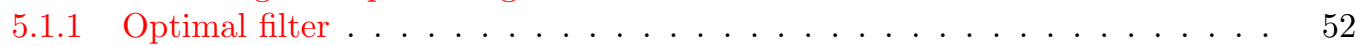

5.1 .2 Optimal signal-to-noise ratio . . . . . . . . . . . . . . . . . 53

5.1 .3 Practical applications of matched filtering . . . . . . . . . . . . . . . 54

5.2 Suboptimal filtering methods . . . . . . . . . . . . . . . . . . 57

5.3 Measurement of parameters and source reconstruction . . . . . . . . . . . . 58

5.3 .1 Ambiguity function . . . . . . . . . . . . . . . . 59

5.3 .2 Metric on the space of waveforms . . . . . . . . . . . . . . 60

5.3 .3 Covariance matrix .......................... 61

5.3 .4 Bayesian inference ........................... 64

6 Physics with Gravitational Waves $\quad 67$

6.1 Speed of gravitational waves . . . . . . . . . . . . . . . . . . . 67

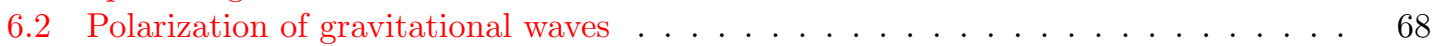

6.3 Gravitational radiation reaction . . . . . . . . . . . . . . . . . . . . . . . . . . . . . . . . . . . . .

6.4 Black hole spectroscopy . . . . . . . . . . . . . . . . . . . . . . 69

6.5 The two-body problem in general relativity . . . . . . . . . . . . . . . . . . . . . 72

6.5.1 Binaries as standard candles: distance estimation . . . . . . . . . . . . . 73

6.5.2 Numerical approaches to the two-body problem . . . . . . . . . . . . 73

6.5.3 Post-Newtonian approximation to the two-body problem . . . . . . . . 75

6.5.4 Measuring the parameters of an inspiraling binary . . . . . . . . . . . 80

6.5.5 Improvement from higher harmonics . . . . . . . . . . . . . . . . 83

6.6 Tests of general relativity . . . . . . . . . . . . . . . . . . . . . . . . . . . . . . . . . . . . . . 84

6.6.1 Testing the post-Newtonian approximation . . . . . . . . . . . . . 84

6.6 .2 Uniqueness of Kerr geometry . . . . . . . . . . . . . . . . . . . . . . 87

6.6.3 Quantum gravity ...................... 89

7 Astrophysics with Gravitational Waves $\quad 91$

7.1 Interacting compact binaries . . . . . . . . . . . . . . . . . . . . . . . 91

7.1.1 Resolving the mass-inclination degeneracy . . . . . . . . . . . . . . 92

7.2 Black hole astrophysics . . . . . . . . . . . . . . . . . . . . . 93

7.2.1 Gravitational waves from stellar-mass black holes . . . . . . . . . . . . . 93

7.2 .2 Stellar-mass black-hole binaries . . . . . . . . . . . . . . . . . . . 93

7.2 .3 Intermediate-mass black holes . . . . . . . . . . . . . . . . . . . . . . . 94



7.3 Neutron star astrophysics . . . . . . . . . . . . . . . . . . . 96

7.3.1 Gravitational collapse and the formation of neutron stars . . . . . . . . . 96

7.3 .2 Neutron-star-binary mergers . . . . . . . . . . . . . . . . . 96

7.3.3 Neutron-star normal mode oscillations . . . . . . . . . . . . . . . . . 97

7.3 .4 Stellar instabilities . . . . . . . . . . . . . . . . . . . . . . . . . . . . . . 97

7.3 .5 Low-mass X-ray binaries . . . . . . . . . . . . . . . . . . . . . . . . 98

7.3.6 Galactic population of neutron stars . . . . . . . . . . . . . . . . . . . 98

7.4 Multimessenger gravitational-wave astronomy . . . . . . . . . . . . . . . . . 99

8 Cosmology with Gravitational Wave Observations 103

8.1 Detecting a stochastic gravitational wave background . . . . . . . . . . . . . 103

8.1.1 Describing a random gravitational wave field . . . . . . . . . . . . . . . . 103

8.1 .2 Observations with gravitational wave detectors . . . . . . . . . . . . 104

8.1.3 Observations with pulsar timing ............... 105 
8.1.4 Observations using the cosmic microwave background . . . . . . . . . 106

8.2 Origin of a random background of gravitational waves . . . . . . . . . . . . . . 106

8.2.1 Gravitational waves from the Big Bang . . . . . . . . . . . . . . 106

8.2.2 Astrophysical sources of a stochastic background . . . . . . . . . . . . 108

8.3 Cosmography: gravitational wave measurements of cosmological parameters . . . . 108

9 Conclusions and Future Directions $r 10$

10 Acknowledgements $r$

$\begin{array}{ll}\text { References } & 113\end{array}$

\section{List of Tables}

1 Noise power spectral densities $S_{h}(f)$ of various interferometers in operation and under construction: GEO600, Initial LIGO (ILIGO), TAMA, VIRGO, Advanced LIGO (ALIGO), Einstein Telescope (ET) and LISA (instrumental noise only). For each detector the noise PSD is given in terms of a dimensionless frequency $x=f / f_{0}$ and rises steeply above a lower cutoff $f_{s} \ldots \ldots \ldots \ldots \ldots$

2 The value of the (squared) distance $d \ell^{2}=r^{2} / \rho^{2}$ for several values of $P$ and the corresponding smallest match that can be expected between templates and the signal at different values of the SNR. . . . . . . . . . . . . . . . . 



\section{A New Window onto the Universe}

The last six decades have witnessed a great revolution in astronomy, driven by improvements in observing capabilities across the electromagnetic spectrum: very large optical telescopes, radio antennas and arrays, a host of satellites to explore the infrared, X-ray, and gamma-ray parts of the spectrum, and the development of key new technologies (CCDs, adaptive optics). Each new window of observation has brought new surprises that have dramatically changed our understanding of the universe. These serendipitous discoveries have included:

- the relic cosmic microwave background radiation (Penzias and Wilson [287]), which has become our primary tool for exploring the Big Bang;

- the fact that quasi-stellar objects are at cosmological distances (Maarten Schmidt [323]), which has developed into the understanding that they are powered by supermassive black holes;

- pulsars (Hewish and Bell [189]), which opened up the study of neutron stars and illuminated one endpoint for stellar evolution;

- X-ray binary systems (Giacconi and collaborators [326]), which now enable us to make detailed studies of black holes and neutron stars;

- gamma-ray bursts coming from immense distances (Klebesadel et al. [216]), which are not fully explained even today;

- the fact that the expansion of the universe is accelerating (two teams [313, 288]), which has led to the hunt for the nature of dark energy.

None of these discoveries was anticipated by the observing team, and in many cases the instruments were built to observe completely different phenomena.

Within a few years another new window on the universe will open up, with the first direct detection of gravitational waves. There is keen interest in observing gravitational waves directly, in order to test Einstein's theory of general relativity and to observe some of the most exotic objects in nature, particularly black holes. But, in addition, the potential of gravitational wave observations to produce more surprises is very high.

The gravitational wave spectrum is completely distinct from, and complementary to, the electromagnetic spectrum. The primary emitters of electromagnetic radiation are charged elementary particles, mainly electrons; because of overall charge neutrality, electromagnetic radiation is typically emitted in small regions, with short wavelengths, and conveys direct information about the physical conditions of small portions of the astronomical sources. By contrast, gravitational waves are emitted by the cumulative mass and momentum of entire systems, so they have long wavelengths and convey direct information about large-scale regions. Electromagnetic waves couple strongly to charges and so are easy to detect but are also easily scattered or absorbed by material between us and the source; gravitational waves couple extremely weakly to matter, making them very hard to detect but also allowing them to travel to us substantially unaffected by intervening matter, even from the earliest moments of the Big Bang.

These contrasts, and the history of serendipitous discovery in astronomy, all suggest that electromagnetic observations may be poor predictors of the phenomena that gravitational wave detectors will eventually discover. Given that $96 \%$ of the mass-energy of the universe carries no charge, gravitational waves provide us with our first opportunity to observe directly a major part of the universe. It might turn out to be as complex and interesting as the charged minor component, the part that we call "normal" matter. 
Several long-baseline interferometric gravitational-wave detectors planned over a decade ago [Laser Interferometer Gravitational-Wave Observatory (LIGO) [18], GEO [244], VIRGO [109] and TAMA [363]] have begun initial operations [3, 245, 19] with unprecedented sensitivity levels and wide bandwidths at acoustic frequencies $(10 \mathrm{~Hz}-10 \mathrm{kHz})$ [197]. These large interferometers are superseding a world-wide network of narrow-band resonant bar antennas that operated for several decades at frequencies near $1 \mathrm{kHz}$. Before 2020 the space-based LISA [71] gravitational wave detector may begin observations in the low-frequency band from $0.1 \mathrm{mHz}$ to $0.1 \mathrm{~Hz}$. This suite of detectors can be expected to open up the gravitational wave window for astronomical exploration, and at the same time perform stringent tests of general relativity in its strong-field dynamic sector.

Gravitational wave antennas are essentially omni-directional, with linearly polarized quadrupolar antenna patterns that typically have a response better than $50 \%$ of its average over $75 \%$ of the sky [197]. Their nearly all-sky sensitivity is an important difference from pointed astronomical antennas and telescopes. Gravitational wave antennas operate as a network, with the aim of taking data continuously. Ground-based interferometers can at present (2008) survey a volume of order $10^{4} \mathrm{Mpc}^{3}$ for inspiraling compact star binaries - among the most promising sources for these detectors - and plan to enhance their range more than tenfold with two major upgrades (to enhanced and then advanced detectors) during the period 2009-2014. For the advanced detectors, there is great confidence that the resulting thousandfold volume increase will produce regular detections. It is this second phase of operation that will be more interesting from the astrophysical point of view, bringing us physical and astrophysical insights into populations of neutron star and black hole binaries, supernovae and formation of compact objects, populations of isolated compact objects in our galaxy, and potentially even completely unexpected systems. Following that, LISA's ability to survey the entire universe for black hole coalescences at milliHertz frequencies will extend gravitational wave astronomy into the cosmological arena.

However, the present initial phase of observation, or observations after the first enhancements, may very well produce the first detections. Potential sources include coalescences of binaries consisting of black holes at a distance of 100-200 Mpc and spinning neutron stars in our galaxy with ellipticities greater than about $10^{-6}$. Observations even at this initial level may, of course, also reveal new sources not observable in any other way. These initial detections, though not expected to be frequent, would be important from the fundamental physics point of view and could enable us to directly test general relativity in the strongly nonlinear regime.

Gravitational wave detectors register gravitational waves coherently by following the phase of the wave and not just measuring its intensity. Since the phase is determined by large-scale motions of matter inside the sources, much of the astrophysical information is extracted from the phase. This leads to different kinds of data analysis methods than one normally encounters in astronomy, based on matched filtering and searches over large parameter spaces of potential signals. This style of data analysis requires the input of pre-calculated template signals, which means that gravitational wave detection depends more strongly than most other branches of astronomy on theoretical input. The better the input, the greater the range of the detectors.

The fact that detectors are omni-directional and detect coherently the phase of the incoming wave makes them in many ways more like microphones for sound than like conventional telescopes. The analogy with sound can be helpful, since microphones can be used to monitor environments for disturbances in any location, and since when we listen to sounds our brains do a form of matched filtering to allow us to interpret the sounds we want to understand against a background of noise. In a very real sense, gravitational wave detectors will be listening to the sounds of a restless universe. The gravitational wave "window" will actually be a listening post, a monitor for the most dramatic events that occur in the universe.

Living Reviews in Relativity

http: //www . livingreviews . org/lrr-2009-2 


\subsection{Birth of gravitational astronomy}

Gravity is the dominant interaction in most astronomical systems. The big surprise of the last three decades of the 20th century was that relativistic gravitation is relevant in so many of these systems. Strong gravitational fields are Nature's most efficient converters of mass into energy. Examples where strong-field relativistic gravity is important include the following:

- neutron stars, the residue of supernova explosions, represent up to $0.1 \%$ (by number) of the entire stellar population of any galaxy;

- stellar-mass black holes power many binary X-ray sources and tend to concentrate near the centers of globular clusters;

- massive black holes in the range $10^{6}-10^{9} M_{\odot}$ seem almost ubiquitous in galaxies that have central bulges, and power active galaxies, quasars, and giant radio jets;

- and, of course, the Big Bang is the only naked singularity we expect to be able to see.

Most of these systems are either dynamical or were formed in catastrophic events; many are or were, therefore, strong sources of gravitational radiation. As the 21st century opens, we are on the threshold of using this radiation to gain a new perspective on the observable universe.

The theory of gravitational radiation already makes an important contribution to the understanding of a number of astronomical systems, such as neutron star binaries, cataclysmic variables, young neutron stars, low-mass X-ray binaries, and even the anisotropy of the microwave background radiation. As the understanding of relativistic phenomena improves, it can be expected that gravitational radiation will play a crucial role as a theoretical tool in modeling relativistic astrophysical systems.

\subsection{What this review is about}

The first three-quarters of the 20th century were required to place the mathematical theory of gravitational radiation on a sound footing. Many of the most fundamental constructs in general relativity, such as null infinity and the theory of conserved quantities, were developed at least in part to help solve the technical problems of gravitational radiation. We will not cover this history here, for which there are excellent reviews [259, 132]. There are still many open questions, since it is impossible to construct exact solutions for most interesting situations. For example, we still lack a full understanding of the two-body problem, and we will review the theoretical work on this problem below. But the fundamentals of the theory of gravitational radiation are no longer in doubt. Indeed, the observation of the orbital decay in the binary pulsar PSR B1913+16 [388] has lent irrefutable support to the correctness of the theoretical foundations aimed at computing gravitational wave emission, in particular to the energy and angular momentum carried away by the radiation.

It is, therefore, to be expected that the evolution of astrophysical systems under the influence of strong tidal gravitational fields will be associated with the emission of gravitational waves. Consequently, these systems are of interest both to a physicist, whose aim is to understand fundamental interactions in nature, their inter-relationships and theories describing them, and to an astrophysicist, who wants to dig deeper into the environs of dense or nonlinearly gravitating systems in solving the mysteries associated with relativistic phenomena listed in Sections 6, 7 and 8. Indeed, some of the gravitational wave antennas that are being built are capable of observing systems to cosmological distances, and even to the edge of the universe. The new window, therefore, is also of interest to cosmologists.

This is a living review of the prospects that lie ahead for gravitational antennas to test the predictions of general relativity as a fundamental theory, for using relativistic gravitation as a 
means to understand highly energetic sources, for interpreting gravitational waves to uncover the (electromagnetically) dark universe, and ultimately for employing networks of gravitational wave detectors to observe the first fraction of a second of the evolution of the universe.

We begin in Section 2 with a brief review of the physical nature of gravitational waves, giving a heuristic derivation of the formulas involved in the calculation of the gravitational wave observables such as the amplitude, frequency and luminosity of gravitational waves. This is followed in Section 3 by a discussion of the astronomical sources of gravitational waves, their expected event rates, amplitudes, waveforms and spectra. In Section 4 we then give a detailed description of the existing and upcoming gravitational wave antennas and their sensitivity. Included in Section 4 are bar and interferometric antennas covering both ground and space-based experiments. Section 4 also compares the sensitivity of the antennas with the strengths of astronomical sources and expected signal-to-noise ratios (SNRs). We then turn in Section 5 to data analysis, which is a central component of gravitational wave astronomy, focusing on those aspects of analysis that are crucial in gleaning physical, astrophysical and cosmological information from gravity wave observations.

Sections 7-9 treat in some detail how gravitational wave observations will aid in a better understanding of nonlinear gravity and test some of its fundamental predictions. In Section 6 we review the physics implications of gravitational wave observations, including new tests of general relativity that can be performed via gravitational wave observations, how these observations may help in formulating and gaining insight into the two-body problem in general relativity, and how gravitational wave observations may help to probe the structure of the universe and the nature of dark energy. In Section 7 we look at the astronomical information returned by gravitational wave observations, and how these observations will affect our understanding of black holes, neutron stars, supernovae, and other relativistic phenomena. Section 8 is devoted to the cosmological implications of gravitational wave observations, including placing constraints on inflation, early phase transitions associated with spontaneous symmetry breaking, and the large-scale structure of the universe.

This review is by no means exhaustive. We plan to expand it to include other key topics in gravitational wave astronomy with subsequent revisions.

Unless otherwise specified we shall use a system of units in which $c=G=1$, which means $1 M_{\odot} \simeq 5 \times 10^{-6} \mathrm{~s} \simeq 1.5 \mathrm{~km}, 1 \mathrm{Mpc} \simeq 10^{14} \mathrm{~s}$. We shall assume a universe with cold dark-matter density of $\Omega_{M}=0.3$, dark energy of $\Omega_{\Lambda}=0.7$, and a Hubble constant of $H_{0}=70 \mathrm{~km} \mathrm{~s}^{-1} \mathrm{Mpc}^{-1}$. 


\section{Gravitational Wave Observables}

To benefit from gravitational wave observations we must first understand what are the attributes of gravitational waves that we can observe. This section is devoted to a short discussion of the nature of gravitational radiation.

\subsection{Gravitational field vs gravitational waves}

Gravitational waves are propagating oscillations of the gravitational field, just as light and radio waves are propagating oscillations of the electromagnetic field. Whereas light and radio waves are emitted by accelerated electrically-charged particles, gravitational waves are emitted by accelerated masses. However, since there is only one sign of mass, gravitational waves never exist on their own: they are never more than a small part of the overall external gravitational field of the emitter. One may wonder, therefore, how it is possible to infer the presence of an astronomical body by the gravitational waves that it emits, when it is clearly not possible to sense its much larger stationary (essentially Newtonian) gravitational potential. There are, in fact, two reasons:

- In general relativity, the effects of both the stationary field and gravitational radiation are described by the tidal forces they produce on free test masses. In other words, single geodesics alone cannot detect gravity or gravitational radiation; we need at least a pair of geodesics. While the stationary tidal force due to the Newtonian potential $\phi$ of a self-gravitating source at a distance $r$ falls off as $\nabla \nabla \phi \sim r^{-3}$, the tidal force due to the gravitational wave amplitude $h$ that it emits at wavelength $\lambda$ decreases as $\nabla \nabla h \sim r^{-1} \lambda^{-2}$. Therefore, the stationary coulomb gravitational potential is the dominant tidal force close to the gravitating body (in the near zone, where $r \leq \lambda)$. However, in the far zone $(r \gg \lambda)$ the tidal effect of the waves is much stronger.

- The stationary part of the tidal field is a DC effect, and simply adds to the stationary tidal forces of all other objects in the universe. It is not possible to discriminate one source from another. Gravitational waves carry time-dependent tidal forces, and so they can be discriminated from the stationary field if one knows what kind of time dependence to look for. Interferometers are ideal detectors in this respect because they sense only changes in the position of an interference fringe, which makes them insensitive to the DC part of the tidal field.

Because gravitational waves couple so weakly to our detectors, those astronomical sources that we can detect must be extremely luminous in gravitational radiation. Even at the distance of the Virgo cluster of galaxies, a detectable source could be as luminous as the full Moon, if only for a millisecond! Indeed, while radio astronomers deal with flux levels of Jy, mJy and even $\mu \mathrm{Jy}$, in the case of gravitational wave sources we encounter fluxes that are typically $10^{20} \mathrm{Jy}$ or larger. Gravitational wave astronomy therefore is biased toward looking for highly energetic, even catastrophic, events.

Extracting useful physical, astrophysical and cosmological information from gravitational wave observations is made possible by measuring a number of gravitational wave attributes that are related to the properties of the source. In the rest of this section we discuss those attributes of gravitational radiation that can be measured via gravitational wave observations. In the process we will review the basic formulas used in computing the gravitational wave amplitude and luminosity of a source. These will then be used in Section 3 to make an order-of-magnitude estimate of the strength of astronomical sources of gravitational waves. 


\subsection{Gravitational wave polarizations}

Because of the equivalence principle, single isolated particles cannot be used to measure gravitational waves: they fall freely in any gravitational field and experience no effects from the passage of the wave. Instead, one must look for inhomogeneities in the gravitational field, which are the tidal forces carried by the waves, and which can be measured only by comparing the positions or interactions of two or more particles.

In general relativity, gravitational radiation is represented by a second rank, symmetric tracefree tensor. In a general coordinate system, and in an arbitrary gauge (coordinate choice), this tensor has ten independent components. However, as in the electromagnetic case, gravitational radiation has only two independent states of polarization in Einstein's theory: the plus polarization and the cross polarization (the names being derived from the shape of the equivalent force fields that they produce). In contrast to electromagnetic waves, the angle between the two polarization states is $\pi / 4$ rather than $\pi / 2$. This is illustrated in Figure 1 , where the response of a ring of free particles in the $(x, y)$ plane to plus-polarized and cross-polarized gravitational waves traveling in the $z$-direction is shown. The effect of the waves is to cause a tidal deformation of the circular ring into an elliptical ring with the same area. This tidal deformation caused by passing gravitational waves is the basic principle behind the construction of gravitational wave antennas.



Figure 1: In Einstein's theory, gravitational waves have two independent polarizations. The effect on proper separations of particles in a circular ring in the $(x, y)$-plane due to a plus-polarized wave traveling in the $z$-direction is shown in (a) and due to a cross-polarized wave is shown in (b). The ring continuously gets deformed into one of the ellipses and back during the first half of a gravitational wave period and gets deformed into the other ellipse and back during the next half.

The two independent polarizations of gravitational waves are denoted $h_{+}$and $h_{\times}$. These are the two primary time-dependent observables of a gravitational wave. The polarization of gravitational waves from a source, such as a binary system, depends on the orientation of the dynamics inside the source relative to the observer. Therefore, measuring the polarization provides information about, for example, the orientation of the binary system.

\subsection{Direction to a source}

Gravitational wave antennas are linearly-polarized quadrupolar detectors and do not have good directional sensitivity. As a result we cannot deduce the direction to a source using a single antenna. One normally needs simultaneous observation using three or more detectors so that the source can be triangulated in the sky by measuring the time differences in signal arrival times at various detectors in a network. Ground-based detectors have typical separation baselines of $L \sim 3 \times 10^{6} \mathrm{~m}$, so that at a wavelength of $\lambda=3 \times 10^{5} \mathrm{~m}=1 \mathrm{~ms}$ (a frequency of $1 \mathrm{kHz}$ ) the network has a resolution of $\delta \theta=\lambda / L=0.1 \mathrm{rad}$. If the amplitude SNR is high, then one can

Living Reviews in Relativity

http://www. livingreviews . org//rr-2009-2 
localize the source by a factor of $1 /$ SNR better than this.

For long-lived sources, however, a single antenna synthesizes many antennas by observing the source at different points along its orbit around the sun. The baseline for such observations is $2 \mathrm{AU}$, so that, for a source emitting radiation at $1 \mathrm{kHz}$, the resolution is as good as $\Delta \theta=10^{-6} \mathrm{rad}$, which is smaller than an arcsecond.

For space-based detectors orbiting the sun, like LISA, the baseline is again $2 \mathrm{AU}$, but the observing frequency is some five or six orders of magnitude lower, so the basic resolution is only of order 1 radian. However, as we shall see later, some of the sources that a space-based detector will observe have huge amplitude SNRs in the range of SNR $\sim 10^{3}-10^{4}$, which improves the resolution to arcminute accuracies in the best cases.

\subsection{Amplitude of gravitational waves - the quadrupole approximation}

The Einstein equations are too difficult to solve analytically in the generic case of a strongly gravitating source to compute the luminosity and amplitude of gravitational waves from an astronomical source. We will discuss numerical solutions later; the most powerful available analytic approach is called the post-Newtonian approximation scheme. This approximation is suited to gravitationallybound systems, which constitute the majority of expected sources. In this scheme [79, 169], solutions are expanded in the small parameter $(v / c)^{2}$, where $v$ is the typical dynamical speed inside the system. Because of the virial theorem, the dimensionless Newtonian gravitational potential $\phi / c^{2}$ is of the same order, so that the expansion scheme links orders in the expanded metric with those in the expanded source terms. The lowest-order post-Newtonian approximation for the emitted radiation is the quadrupole formula, and it depends only on the density $(\rho)$ and velocity fields of the Newtonian system. If we define the spatial tensor $Q_{j k}$, the second moment of the mass distribution, by the equation

$$
Q_{j k}=\int \rho x_{j} x_{k} \mathrm{~d}^{3} x,
$$

then the amplitude of the emitted gravitational wave is, at lowest order, the three-tensor

$$
h_{j k}=\frac{2}{r} \frac{d^{2} Q_{j k}}{d t^{2}} .
$$

This is to be interpreted as a linearized gravitational wave in the distant almost-flat geometry far from the source, in a coordinate system (gauge) called the Lorentz gauge.

\subsubsection{Wave amplitudes and polarization in TT-gauge}

A useful specialization of the Lorentz gauge is the TT-gauge, which is a comoving coordinate system: free particles remain at constant coordinate locations, even as their proper separations change. To get the TT-amplitude of a wave traveling outwards from its source, project the tensor in Equation (2) perpendicular to its direction of travel and remove the trace of the projected tensor. The result of doing this to a symmetric tensor is to produce, in the transverse plane, a two-dimensional matrix with only two independent elements:

$$
h_{a b}=\left(\begin{array}{cc}
h_{+} & h_{\times} \\
h_{\times} & -h_{+}
\end{array}\right) .
$$

This is the definition of the wave amplitudes $h_{+}$and $h_{\times}$that are illustrated in Figure 1 . These amplitudes are referred to as the coordinates chosen for that plane. If the coordinate unit basis vectors in this plane are $\hat{e}_{x}$ and $\hat{e}_{y}$, then we can define the basis tensors

$$
\begin{aligned}
& \mathbf{e}_{+}=\hat{e}_{x} \otimes \hat{e}_{x}-\hat{e}_{y} \otimes \hat{e}_{y}, \\
& \mathbf{e}_{\times}=\hat{e}_{x} \otimes \hat{e}_{y}+\hat{e}_{y} \otimes \hat{e}_{x} .
\end{aligned}
$$


In terms of these, the TT-gravitational wave tensor can be written as

$$
\mathbf{h}=h_{+} \mathbf{e}_{+}+h_{\times} \mathbf{e}_{\times}
$$

If the coordinates in the transverse plane are rotated by an angle $\psi$, then one obtains new amplitudes $h_{+}^{\prime}$ and $h_{\times}^{\prime}$ given by

$$
\begin{aligned}
& h_{+}^{\prime}=\cos 2 \psi h_{+}+\sin 2 \psi h_{\times}, \\
& h_{\times}^{\prime}=-\sin 2 \psi h_{+}+\cos 2 \psi h_{\times} .
\end{aligned}
$$

This shows the quadrupolar nature of the polarizations, and is consistent with our remark in association with Figure 1 that a rotation of $\pi / 4$ changes one polarization into the other.

It should be clear from the TT projection operation that the emitted radiation is not isotropic: it will be stronger in some directions than in others ${ }^{1}$. It should also be clear from this that spherically-symmetric motions do not emit any gravitational radiation: when the trace is removed, nothing remains.

\subsubsection{Simple estimates}

A typical component of $d^{2} Q_{j k} / d t^{2}$ will (from Equation (1)) have magnitude $\left(M v^{2}\right)_{\text {nonsph }}$, where $\left(M v^{2}\right)_{\text {nonsph }}$ is twice the nonspherical part of the kinetic energy inside the source. So a bound on any component of Equation (2) is

$$
h \lesssim \frac{2\left(M v^{2}\right)_{\text {nonsph }}}{r}
$$

It is interesting to observe that the ratio $\epsilon$ of the wave amplitude to the Newtonian potential $\phi_{\text {ext }}$ of its source at the observer's distance $r$ is simply bounded by

$$
h / \phi_{\text {ext }}<2 v_{\text {nonsph }}^{2}
$$

and this bound is attained if the entire mass of the source is involved in the nonspherical motions, so that $\left(M v^{2}\right)_{\text {nonsph }} \sim M v_{\text {nonsph }}^{2}$. By the virial theorem for self-gravitating bodies

$$
v_{\text {nonsph }}^{2} \leq \phi_{\text {int }}
$$

where $\phi_{\text {int }}$ is the maximum value of the Newtonian gravitational potential inside the system. This provides a convenient bound in practice [328]:

$$
h \lesssim 2 \phi_{\text {int }} \phi_{\text {ext }}
$$

The bound is attained if the system is highly nonspherical. An equal-mass star binary system is a good example of a system that attains this bound.

For a neutron star source, one has $\phi_{\text {int }} \sim 0.2$. If the star is in the Virgo cluster $(r \sim 18 \mathrm{Mpc})$ and has a mass of $1.4 M_{\odot}$, and if it is formed in a highly-nonspherical gravitational collapse, then the upper limit on the amplitude of the radiation from such an event is $1.5 \times 10^{-21}$. This is a simple way to get the number that has been the goal of detector development for decades, to make detectors that can observe waves at or below an amplitude of about $10^{-21}$.

\footnotetext{
${ }^{1}$ In the case of an inspiraling binary, the root mean square of the two polarization amplitudes in a direction orthogonal to the orbital plane will be a factor $2 \sqrt{2}$ larger than in the plane.
} 


\subsection{Frequency of gravitational waves}

The signals for which the best waveform predictions are available have well-defined frequencies. In some cases the frequency is dominated by an existing motion, such as the spin of a pulsar. But in most cases the frequency will be related to the natural frequency for a self-gravitating body, defined as

$$
\omega_{0}=\sqrt{\pi G \bar{\rho}}, \text { or } f_{0}=\omega_{0} / 2 \pi=\sqrt{G \bar{\rho} / 4 \pi},
$$

where $\bar{\rho}$ is the mean density of mass-energy in the source. This is of the same order as the binary orbital frequency and the fundamental pulsation frequency of the body. Even though this is a Newtonian formula, it provides a remarkably good order-of-magnitude prediction of natural frequencies, even for highly relativistic systems such as black holes.

The frequency of the emitted gravitational waves need not be the natural frequency, of course, even if the mechanism is an oscillation with that frequency. In many cases, such as binary systems, the radiation comes out at twice the oscillation frequency. But since, at this point, we are not trying to be more accurate than a few factors, we will ignore this distinction here. In later sections, with specific source models, we will get the factors right.

The mean density and hence the frequency are determined by the size $R$ and mass $M$ of the source, taking $\bar{\rho}=3 M / 4 \pi R^{3}$. For a neutron star of mass $1.4 M_{\odot}$ and radius $10 \mathrm{~km}$, the natural frequency is $f_{0}=1.9 \mathrm{kHz}$. For a black hole of mass $10 M_{\odot}$ and radius $2 M=30 \mathrm{~km}$, it is $f_{0}=1 \mathrm{kHz}$. And for a large black hole of mass $2.5 \times 10^{6} M_{\odot}$, such as the one at the center of our galaxy, this goes down in inverse proportion to the mass to $f_{0}=4 \mathrm{mHz}$. In general, the characteristic frequency of the radiation of a compact object of mass $M$ and radius $R$ is

$$
f_{0}=\frac{1}{4 \pi}\left(\frac{3 M}{R^{3}}\right)^{1 / 2} \simeq 1 \mathrm{kHz}\left(\frac{10 M_{\odot}}{M}\right) .
$$

Figure 2 shows the mass-radius diagram for likely sources of gravitational waves. Three lines of constant natural frequency are plotted: $f_{0}=10^{4} \mathrm{~Hz}, f_{0}=1 \mathrm{~Hz}$, and $f_{0}=10^{-4} \mathrm{~Hz}$. These are interesting frequencies from the point of view of observing techniques: gravitational waves between 1 and $10^{4} \mathrm{~Hz}$ are in principle accessible to ground-based detectors, while lower frequencies are observable only from space. Also shown is the line marking the black-hole boundary. This has the equation $R=2 M$. There are no objects below this line, because they would be smaller than the horizon size for their mass. This line cuts through the ground-based frequency band in such a way as to restrict ground-based instruments to looking at stellar-mass objects. No system with a mass above about $10^{4} M_{\odot}$ can produce quadrupole radiation in the ground-based frequency band.

A number of typical relativistic objects are placed in the diagram: a neutron star, a pair of neutron stars that spiral together as they orbit, some black holes. Two other interesting lines are drawn. The lower (dashed) line is the 1-year coalescence line, where the orbital shrinking timescale due to gravitational radiation backreaction (cf. Equation (28)) is less than one year. The upper (solid) line is the 1-year chirp line: if a binary lies below this line, then its orbit will shrink enough to make its orbital frequency increase by a measurable amount in one year. (In a one-year observation one can, in principle, measure changes in frequency of $1 \mathrm{yr}^{-1}$, or $3 \times 10^{-8} \mathrm{~Hz}$.)

It is clear from the Figure that any binary system that is observed from the ground will coalesce within an observing time of one year. Since pulsar binary statistics suggest that neutronstar-binary coalescences happen less often than once every $10^{5}$ years in our galaxy, ground-based detectors must be able to register these events in a volume of space containing at least $10^{6}$ galaxies in order to have a hope of seeing occasional coalescences. That corresponds to a volume of radius roughly $100 \mathrm{Mpc}$. For comparison, first-generation ground-based interferometric detectors have a reach of around $20 \mathrm{Mpc}$ for such binaries, while advanced interferometers should extend that to about $200 \mathrm{Mpc}$. 


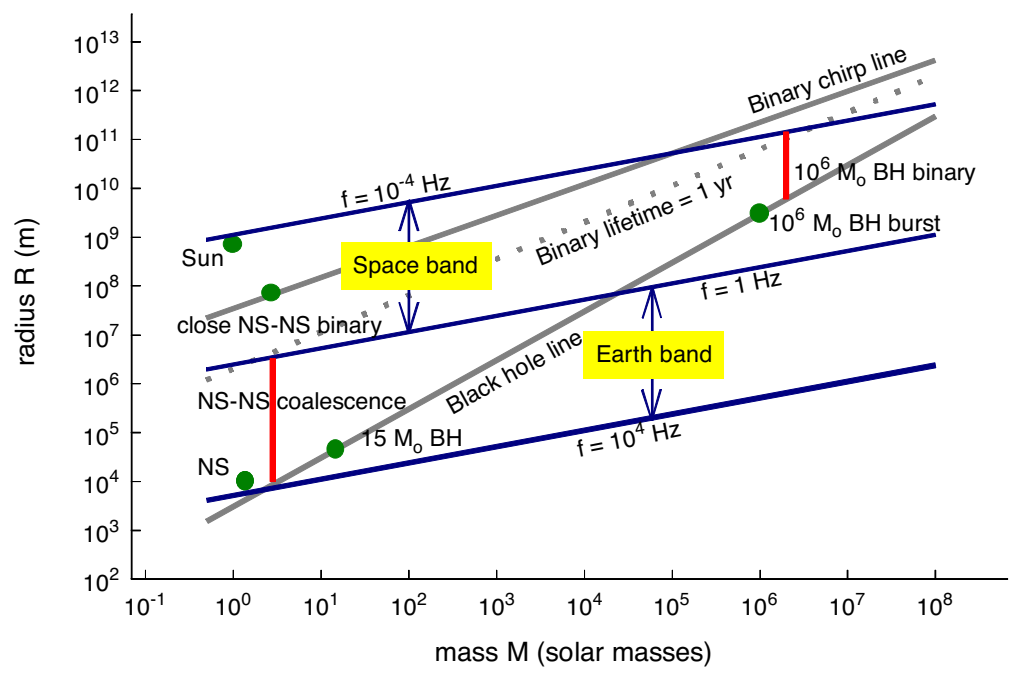

Figure 2: Mass-radius plot for gravitational wave sources. The horizontal axis is the total mass of a radiating system, and the vertical axis is its size. Typical values from various sources for ground-based and space-based detectors are shown. Lines give order-of-magnitude constraints and relations. Characteristic frequencies are estimated from $f \sim(G \rho / 4 \pi)^{1 / 2}$. The black-hole and binary lines are described in the text.

\subsection{Luminosity in gravitational waves}

The general formula for the local stress-energy of a gravitational wave field propagating through flat spacetime, using the TT-gauge, is given by the Isaacson expression [259, 332]

$$
T_{\alpha \beta}=\frac{1}{32 \pi}\left\langle h_{j k, \alpha}^{\mathrm{TT}} h_{, \beta}^{\mathrm{TT} j k}\right\rangle,
$$

where the angle brackets denote averages over regions of the size of a wavelength and times of the length of a period of the wave. The energy flux of a wave in the $x^{i}$ direction is the $T^{0 i}$ component.

The gravitational wave luminosity in the quadrupole approximation is obtained by integrating the energy flux from Equation (14) over a distant sphere. When one correctly takes into account the projection factors mentioned after Equation (2), one obtains [259]

$$
L_{g w}=\frac{1}{5}\left(\sum_{j, k} \dddot{Q}_{j k} \dddot{Q}_{j k}-\frac{1}{3} \dddot{Q}^{2}\right),
$$

where $Q$ is the trace of the matrix $Q_{j k}$. This equation can be used to estimate the backreaction effect on a system that emits gravitational radiation.

Notice that the expression for $L_{g w}$ is dimensionless when $c=G=1$. It can be converted to normal luminosity units by multiplying by the scale factor

$$
L_{0}=c^{5} / G=3.6 \times 10^{52} \mathrm{~W} .
$$

This is an enormous luminosity. By comparison, the luminosity of the sun is only $3.8 \times 10^{26} \mathrm{~W}$, and that of a typical galaxy would be $10^{37} \mathrm{~W}$. All the galaxies in the visible universe emit, in visible light, on the order of $10^{49} \mathrm{~W}$. We will see that gravitational wave systems always emit at a fraction of $L_{0}$, but that the gravitational wave luminosity can come close to $L_{0}$ and can greatly exceed typical electromagnetic luminosities. Close binary systems normally radiate much more 
energy in gravitational waves than in light. Black hole mergers can, during their peak few cycles, compete in luminosity with the steady luminosity of the entire universe!

Combining Equations (2) and (15) one can derive a simple expression for the apparent luminosity of radiation $\mathcal{F}$, at great distances from the source, in terms of the gravitational wave amplitude [332]:

$$
\mathcal{F} \sim \frac{|\dot{h}|^{2}}{16 \pi} .
$$

The above relation can be used to make an order-of-magnitude estimate of the gravitational wave amplitude from a knowledge of the rate at which energy is emitted by a source in the form of gravitational waves. If a source at a distance $r$ radiates away energy $E$ in a time $T$, predominantly at a frequency $f$, then writing $\dot{h}=2 \pi f h$ and noting that $\mathcal{F} \sim E /\left(4 \pi r^{2} T\right)$, the amplitude of gravitational waves is

$$
h \sim \frac{1}{\pi f r} \sqrt{\frac{E}{T}} .
$$

When the time development of a signal is known, one can filter the detector output through a copy of the expected signal (see Section 5 on matched filtering). This leads to an enhancement in the SNR, as compared to its narrow-band value, by roughly the square root of the number of cycles the signal spends in the detector band. It is useful, therefore, to define an effective amplitude of a signal, which is a better measure of its detectability than its raw amplitude:

$$
h_{\mathrm{eff}} \equiv \sqrt{n} h .
$$

Now, a signal lasting for a time $T$ around a frequency $f$ would produce $n \simeq f T$ cycles. Using this we can eliminate $T$ from Equation (18) and get the effective amplitude of the signal in terms of the energy, the emitted frequency and the distance to the source:

$$
h_{\mathrm{eff}} \sim \frac{1}{\pi r} \sqrt{\frac{E}{f}} .
$$

Notice that this depends on the energy only through the total fluence, or time-integrated flux $E / 4 \pi r^{2}$ of the wave. As in many other branches of astronomy, the detectability of a source is ultimately a function of its apparent luminosity and the observing time. However, one should not ignore the dependence on frequency in this formula. Two sources with the same fluence are not equally easy to detect if they are at different frequencies: higher frequency signals have smaller amplitudes. 


\section{Sources of Gravitational Waves}

\subsection{Man-made sources}

One source can unfortunately be ruled out as undetectable: man-made gravitational radiation. Imagine creating a wave generator with the following extreme properties. It consists of two masses of $10^{3} \mathrm{~kg}$ each (a small car) at opposite ends of a beam $10 \mathrm{~m}$ long. At its center the beam pivots about an axis. This centrifuge rotates 10 times per second. All the velocity is nonspherical, so $v_{\text {nonsph }}^{2}$ in Equation (9) is about $10^{5} \mathrm{~m}^{2} \mathrm{~s}^{-2}$. The frequency of the waves will actually be $20 \mathrm{~Hz}$, since the mass distribution of the system is periodic in time with a period of half the rotation period. The wavelength of the waves will, therefore, be $1.5 \times 10^{7} \mathrm{~m}$, similar to the diameter of the earth. In order to detect gravitational waves, not near-zone Newtonian gravity, the detector must be at least one wavelength from the source, say diametrically opposite the centrifuge on the Earth. Then the amplitude $h$ can be deduced from Equation (9): $h \sim 5 \times 10^{-43}$. This is far too small to contemplate detecting! The story changes, fortunately, when we consider astrophysical sources of gravitational waves, where nature arranges for masses that are $10^{27}$ times larger than our centrifuge to move at speeds close to the speed of light!

Until observations of gravitational waves are successfully made, one can only make intelligent guesses about most of the sources that will be seen. There are many that could be strong enough to be seen by the early detectors: star binaries, supernova explosions, neutron stars, the early universe. In this section, we make rough luminosity estimates using the quadrupole formula and other approximations, which are usually accurate to within factors of order two, and, very importantly, they show how key observables scale with the properties of the systems. Where appropriate we also make use of predictions from the much more accurate modelling that is available for some sources, such as binary systems and black hole mergers. The detectability depends, of course, not only on the intrinsic luminosity of the source, but on how far away it is. Often the biggest uncertainties in making predictions are the spatial density and event rate of any particular class of sources. This is not surprising, since our information at present comes from electromagnetic observations, and as our earlier remarks about the differences between the mechanisms of emission of gravitational and electromagnetic radiation make clear, electromagnetic observations may not strongly constrain the source population.

\subsection{Gravitational wave bursts from gravitational collapse}

Neutron stars and black holes are formed from the gravitational collapse of a highly evolved star or the core collapse of an accreting white dwarf. In either case, if the collapse is nonspherical, perhaps induced by strong rotation, then gravitational waves could carry away some of the binding energy and angular momentum depending on the geometry of the collapse. Collapse events are thought to produce supernovae of various types, and increasingly there is evidence that they also produce most of the observed gamma-ray bursts [191] in hypernovae and collapsars [397, 249]. Supernovae of Type II are believed to occur at a rate of between 0.1 and 0.01 per year in a milkyway equivalent galaxy (MWEG); thus, within the Virgo supercluster, we might expect an event rate of about 30 per year. Hypernova events are considerably rarer and might only contribute observable gravitational-wave events in current and near-future detectors if they involve so much rotation that strong non-axisymmetric instabilities are triggered.

Simulating gravitational collapse is a very active area of numerical astrophysics, and most simulations also predict the energy and spectral characteristics of the emitted gravitational waves [167]. However, it is still beyond the capabilities of computers to simulate a gravitational collapse event with all the physics that might be necessary to give reliable predictions: three-dimensional hydrodynamics, neutrino transport, realistic nuclear physics, magnetic fields, rotation. In fact, it is still by no means clear why Type II supernovae explode at all: simulations typically have great

Living Reviews in Relativity

http: //www. livingreviews.org/lrr-2009-2 
difficulty reversing the inflow and producing an explosion with the observed light-curves and energetics. It may be that the answer lies in some of the physics that has to be oversimplified in order to be used in current simulations, or in some neutrino physics that we do not yet know, or in some unexplored hydrodynamic mechanism [276]. In a typical supernova, simulations suggest that gravitational waves might extract between about $10^{-7}$ and $10^{-5}$ of the total available massenergy [264, 147, 148], and the waves could come off in a burst whose frequency might lie in the range of $\sim 200-1000 \mathrm{~Hz}$.

We can use Equation (18) to make a rough estimate of the amplitude, if the emitted energy and timescale are known. Using representative values for a supernova in our galaxy, lying at $10 \mathrm{kpc}$, emitting the energy equivalent of $10^{-7} M_{\odot}$ at a frequency of $1 \mathrm{kHz}$, and lasting for $1 \mathrm{~ms}$, the received amplitude would be

$$
h \sim 6 \times 10^{-21}\left(\frac{E}{10^{-7} M_{\odot}}\right)^{1 / 2}\left(\frac{1 \mathrm{~ms}}{T}\right)^{1 / 2}\left(\frac{1 \mathrm{kHz}}{f}\right)\left(\frac{10 \mathrm{kpc}}{r}\right) .
$$

The upper bound in Equation (11) would give the same amplitude for a source 60 times further away, which reflects the fact that simulations find it difficult to put significant energy into gravitational waves. This amplitude is large enough for current ground-based detectors to observe with a reasonably high confidence, but of course the event rate within $10 \mathrm{kpc}$ is expected to be far too small to make an early detection likely.

\subsection{Gravitational wave pulsars}

Some likely gravitational wave sources behave like the centrifuge example we used in the first paragraph of this section, only on a grander scale. Suppose a neutron star of radius $R$ and mass $M$ spins with a frequency $f$ and has an irregularity, a deformation of its otherwise axially symmetric shape. We idealize this as a "bump" of mass $m$ on its surface, although of course it will really be a distribution of mass leading to an asymmetrical quadrupole tensor. The moment of inertia of the bump will be $m R^{2}$, and it is conventional to parameterize the bump in terms of the fractional asymmetry it creates in the moment of inertia tensor itself. If we idealize the star as having uniform density, then the spherical moment of inertia is $2 M R^{2} / 5$, and so the bump has fractional asymmetry

$$
\epsilon=\frac{5}{2} \frac{m}{M}, \quad m=0.4 \epsilon M .
$$

The bump will emit gravitational radiation at frequency $2 f$ because the star spins about its net center of mass, so it effectively has mass excesses on both sides of the star. The nonspherical velocity will be just $v_{\text {nonsph }}=2 \pi R f$. The radiation amplitude will be, from Equation (9),

$$
h_{\text {bump }} \sim(4 / 5)(2 \pi R f)^{2} \epsilon M / r,
$$

and the luminosity, from Equation (15) (assuming that roughly four comparable components of $Q_{j k}$ contribute to the sum),

$$
L_{\text {bump }} \sim(16 / 125)(2 \pi f)^{6} \epsilon^{2} M^{2} R^{4} .
$$

The radiated energy would presumably come from the rotational energy of the star $M v^{2} / 5$. This would lead to a spindown of the star on a timescale

$$
t_{\text {spindown }} \sim \frac{1}{5} M v^{2} / L_{\text {bump }} \sim \frac{25}{32 \pi} \epsilon^{-2} f^{-1}\left(\frac{M}{R}\right)^{-1} v^{-3} .
$$

It is believed that neutron star crusts are not strong enough to support fractional asymmetries larger than about $\epsilon \sim 10^{-6}$ [370], and realistic asymmetries may be much smaller. 
From these considerations one can estimate the likelihood that the observed spindown timescales of pulsars are due to gravitational radiation. In most cases, it seems that gravitational wave losses could account for a substantial amount of the spindown: the required asymmetries are much smaller than $10^{-4}$, often smaller than $10^{-7}$. But an interesting exception is the Crab pulsar, PSR J0534+2200, whose young age and consequently short spindown time (measured to be $8.0 \times$ $10^{10} \mathrm{~s}$, about $2500 \mathrm{yr}$ ) would require an exceptionally large asymmetry. If we take the neutron star's radius to be $10 \mathrm{~km}$, so that $M / R \sim 0.21$ and the speed of any irregularity is $v / c \sim 6.2 \times 10^{-3}$, then Equation (24) would require an asymmetry of $\epsilon \sim 1.4 \times 10^{-3}$. Of course, we have made a lot of approximations to get here, only keeping our estimates of amplitudes and energies correct to within factors of two, but a more careful calculation reduces this only by a factor of two to $\epsilon \sim 7 \times 10^{-4}$ [12]. What makes this interesting is the fact that an asymmetry this large would produce radiation detectable by first-generation interferometers. Conversely, an upper limit from first-generation interferometers would provide direct observational limits on the asymmetry and on the fraction of energy lost by the Crab pulsar to gravitational waves.

From Equation (23) the Crab pulsar would, if its spindown is dominated by gravitational wave losses, produce an amplitude at the Earth of $h \sim 1.5 \times 10^{-24}$, if its distance is $2 \mathrm{kpc}$. Is this detectable when present instruments are only capable of seeing millisecond bursts of radiation at levels of $10^{-21}$ ? The answer is yes, if the observation time is long enough. Indeed, the latest LIGO observations have not detected any gravitational waves from the Crab pulsar, which has been used to set an upper limit on the asymmetry in its mass distribution [12]. The limit depends on the model assumed for the pulsar. If one assumes that gravitational waves are produced at exactly twice the pulsar spin frequency and uses the inferred values of the pulsar orientation and polarization angle, then for a canonical value of the moment-of-inertia $I=10^{38} \mathrm{~kg} \mathrm{~m}^{2}$, one gets an upper limit on the ellipticity of $\epsilon \leq 1.8 \times 10^{-4}$, assuming the pulsar is at $2 \mathrm{kpc}$. This is a factor of 4.2 below the spindown limit [12]. If, however, one assumes that gravitational waves are emitted at a frequency close, but not exactly equal, to twice the spin frequency and one uses a uniform prior for the orientation and polarization angle, then one gets $\epsilon \leq 9 \times 10^{-4}$, which is 0.8 of the limit derived from the spin-down rate.

Indeed, even signals weaker than the amplitude determined by the Crab spindown rate will be observable by present detectors, and these may be coming from a larger variety of neutron stars, in particular low-mass X-ray binary systems (LMXBs). The neutron stars in them are accreting mass and angular momentum, so they should be spinning up. Observations suggest that most neutron stars are spinning at speeds between about 300 and $600 \mathrm{~Hz}$, far below their maximum, which is greater than $1000 \mathrm{~Hz}$. The absence of faster stars suggests that something stops them from spinning up beyond this range. Bildsten suggested [77] that the limiting mechanism may be the re-radiation of the accreted angular momentum in gravitational waves, possibly due to a quadrupole moment created by asymmetrical heating induced by the accreted matter. Another possible mechanism [285] is that a "bump" of the kind we have treated is formed by accreting matter channeled onto the surface by the star's magnetic field. It is also possible that accretion drives an instability in the star that leads to steady emission [308, 270]. In either case, the stars could turn out to be long-lived sources of gravitational waves. This idea, which is a variant of one proposed long ago by Wagoner [383], is still speculative, but the numbers make a plausible case. We discuss it in more detail in Section 7.3.5.

Living Reviews in Relativity

http://www. livingreviews. org/lrr-2009-2 


\subsection{Radiation from a binary star system}

\subsubsection{Radiation from a binary system and its backreaction}

A binary star system can also be treated as a "centrifuge". Two stars of the same mass $m$ in a circular orbit of radius $R$ have all their mass in nonspherical motion, so that

$$
\left(M v^{2}\right)_{\text {nonsph }}=M(\Omega R)^{2}=\frac{M^{2}}{R},
$$

where $\Omega$ is the orbital angular velocity. The gravitational wave amplitude can then be written

$$
h_{\text {binary }} \sim 2 \frac{M}{r} \frac{M}{R} .
$$

Since the internal radius $R$ of the orbit is not an observable, it is sometimes convenient to replace $R$ by the orbital angular frequency $\Omega$ using the above orbit equation, giving

$$
h_{\text {binary }} \sim \frac{2}{r} M^{5 / 3} \Omega^{2 / 3} .
$$

The gravitational wave luminosity of such a system is, by a calculation analogous to that for bumps on neutron stars (assuming that four components of $Q_{i j}$ to be significant),

$$
L_{\text {binary }} \sim \frac{4}{5}\left(\frac{M}{R}\right)^{5}
$$

in units given by the fundamental luminosity $L_{0}$ in Equation (16). This shows that self-gravitating systems always emit at a fraction of $L_{0}$, since $M / R$ is always smaller than 1 , but it can approach $L_{0}$ for highly-relativistic systems where $M / R \sim 1$.

The radiation of energy by the orbital motion causes the orbit to shrink. The shrinking will make any observed gravitational waves increase in frequency with time. This is called a chirp. The timescale $^{2}$ for this in a binary system with equal masses is

$$
t_{\text {chirp }}=\frac{M v^{2}}{2} / L_{\text {binary }} \sim \frac{5 M}{8}\left(\frac{M}{R}\right)^{-4} .
$$

As the binary evolves, the frequency and amplitude of the wave grow and this drives the binary to evolve even more rapidly. The signal's frequency, however, will not increase indefinitely; the slow inspiral phase ends either when the stars begin to interact and merge or (if they are very compact) when the distance between the stars is roughly at the last stable orbit (LSO) $R=6 M$, which corresponds to a gravitational wave frequency of

$$
f_{\mathrm{LSO}} \sim 220\left(\frac{20 M_{\odot}}{M}\right) \mathrm{Hz},
$$

where we have normalized this to a binary with $M=20 M_{\odot}$. This is the last stable orbit (LSO) frequency.

A compact-object binary that coalesces after passing through the last stable orbit is a powerful source of gravitational waves, with a luminosity that approaches the limiting luminosity $L_{0}$. This is called a coalescing binary in gravitational wave searches. Since a typical search might last on the order of one year, a coalescing binary can be defined as a system that has a chirp time smaller

\footnotetext{
${ }^{2}$ In Sections 5.1 we will use parameters called chirp times, instead of the masses, to characterize a binary. The timescale defined here is closely related to the chirp times.
} 
than one year. In Figure 2 the coalescence line is shown as a straight line with slope $3 / 4$ (set $t_{\text {chirp }}$ to a constant in Equation (28)). Binary systems below this line have a chirp time smaller than one year. It is evident from the figure that all binary systems observable by ground-based detectors will coalesce in less than a year.

As mentioned for gravitational wave pulsars, the raw amplitude of the radiation from a longlived system is not by itself a good guide to its detectability, if the waveform can be predicted. Data analysis techniques like matched filtering are able to eliminate most of the detector noise and allow the recognition of weaker signals. The improvement in amplitude sensitivity is roughly proportional to the square root of the number of cycles of the waveform that one observes. For neutron stars that are observed from a frequency of $10 \mathrm{~Hz}$ until they coalesce, there could be on the order of $10^{4}$ cycles, meaning that the sensitivity of a second-generation interferometric detector would effectively be 100 times better than its broadband (prefiltering) sensitivity. Such detectors could see typical coalescences at $\sim 200 \mathrm{Mpc}$. The event rate for second-generation detectors is estimated at around 40 events per year, with rather large error bars [101, 211, 242].

\subsubsection{Chirping binaries as standard sirens}

When we consider real binaries we must do the calculation for systems that have unequal masses. Still assuming for the moment that the binary orbit is circular, the quadrupole amplitude turns out to be

$$
h_{\text {binary }} \sim \frac{1}{r} \mathcal{M}^{5 / 3} \Omega^{2 / 3}
$$

where we define the chirp mass $\mathcal{M}$ as

$$
\mathcal{M}=\mu^{3 / 5} M^{2 / 5}=\nu^{3 / 5} M, \quad \nu=\frac{\mu}{M},
$$

with $\mu$ the reduced mass, $M$ the total mass and $\nu$ the symmetric mass ratio. We have left out of Equation (30) a factor of order one that depends on the angle from which the binary system is viewed. The two polarization amplitudes can be found in Equation (132).

Remarkably, the other observable, namely the shrinking of the orbit as measured by the rate of change of the orbital frequency $P_{b}$ also depends on the masses just through $\mathcal{M}$ [290]:

$$
\dot{P}_{b}=-\frac{192 \pi}{5}\left(\frac{2 \pi \mathcal{M}}{P_{b}}\right)^{5 / 3}
$$

In this case, the chirp time is

$$
t_{\text {chirp }}=\frac{5 M}{96} \frac{1}{\nu}\left(\frac{M}{R}\right)^{-4} .
$$

This is just the equal-mass chirp time of Equation (28) scaled inversely with the symmetric mass ratio $\nu=m_{1} m_{2} / M^{2}$. From this equation it is clear that systems with large mass ratios between the components can spend a long time in highly relativistic orbits, whereas equal-mass binaries can be expected to merge after only a few orbits in the highly relativistic regime.

If one observes $P_{b}$ and $\dot{P}_{b}$, one can infer $\mathcal{M}$ from Equation (32). Then, from the observed amplitude in Equation (30), the only remaining unknown is the distance $r$ to the source. Gravitational wave observations of orbits that shrink because of gravitational energy losses can therefore directly determine the distance to the source [329]. By analogy with the "standard candles" of electromagnetic astronomy, these systems are now being called "standard sirens". Although our calculation here assumed an equal-mass circular system, the conclusion is robust: any binary, even with ellipticity and extreme mass ratio, encodes its distance in its gravitational wave signal.

This is another way in which gravitational wave observations are complementary to electromagnetic ones, providing information that is hard to obtain electromagnetically. One consequence is 
the possibility that observations of coalescing compact object binaries could allow one to measure the Hubble constant [329] or other cosmological parameters. This will be particularly interesting for the LISA project, whose observations of black hole binaries could contribute an independent measurement of the acceleration of the universe [195, 131, 48].

Because chirping systems are so interesting we have also drawn, in Figure 2, a line where the chirp time can be measured in one year. This means that the change in frequency due to the chirp must be larger than the frequency resolution $1 \mathrm{yr}^{-1}$. A little algebra shows that the condition for the chirp to be resolved in an observation time $T$ in a binary with period $P_{b}$ is

$$
P_{b} t_{\text {chirp }}=T^{2} .
$$

Since $P_{b} \propto R^{3 / 2} M^{-1 / 2}$, this condition leads to a line of slope $7 / 11$ in the logarithmic plot in Figure 2. The line drawn there corresponds to a resolution time $T$ of one year. All binaries below this line will chirp in a short enough time for their distances to be measured.

\subsubsection{Binary pulsar tests of gravitational radiation theory}

The most famous example of the effects of gravitational radiation on an orbiting system is the Hulse-Taylor Binary Pulsar, PSR B1913+16. In this system, two neutron stars orbit in a close eccentric orbit. The pulsar provides a regular clock that allows one to deduce, from post-Newtonian effects, all the relevant orbital parameters and the masses of the stars. The key to the importance of this binary system is that all of the important parameters of the system can be measured before one takes account of the orbital shrinking due to gravitational radiation reaction. This is because a number of post-Newtonian effects on the arrival time of pulses at the Earth, such as the precession of the position of the periastron and the time-dependent gravitational redshift of the pulsar period as it approaches and recedes from its companion, can be measured accurately, and they fully determine the masses, the semi-major axis and the eccentricity of their orbit [394, 344].

Equation (28) for the chirp time predicts that this system would change its orbital period $P_{b}=7.75 \mathrm{hrs}$ on the timescale (taking $M=1.4 M_{\odot}$ and $R=10^{6} \mathrm{~km}$ )

$$
t_{\text {chirp }}=P_{b} / \dot{P}_{b} \sim 1.9 \times 10^{18} \mathrm{~s} .
$$

From this one can infer that $\dot{P}_{b} \sim 1.5 \times 10^{-14}$. But this has to be corrected for our oversimplification of the orbit as circular: an eccentric orbit evolves much faster because, during the phase of closest approach, the velocities are much higher, and the emitted luminosity is a very strong function of the velocity. Using equations first computed by Peters and Mathews [290], for the actual eccentricity of 0.62 , one finds (see Equation (109) below) $\dot{P}_{\mathrm{T}}=-(2.40242 \pm 0.00002) \times 10^{-12}$. Observations [394, 388] currently give $\dot{P}_{\mathrm{O}}=-(2.4184 \pm 0.0009) \times 10^{-12}$. There is a significant discrepancy between these, but it can be removed by realizing that the binary system is accelerating toward the center of our galaxy, which produces a small period change. Taking this into account gives a corrected prediction of $-(2.4056 \pm 0.0051) \times 10^{-12}$, and this agrees with the observation within the uncertainties [394, 355]. This is the most sensitive test that we have of the correctness of Einstein's equations with respect to gravitational radiation, and it leaves little room for doubt in the validity of the quadrupole formula for other systems that may generate detectable radiation.

A number of other binary systems are now known in which such tests are possible [344]. The most important of the other systems is the "double pulsar" in which both neutron stars are seen as pulsars [246]. This system will soon overtake the Hulse-Taylor binary as the most accurate test of gravitational radiation.

\subsubsection{White-dwarf binaries}

Binary systems at lower frequencies are much more abundant than coalescing binaries, and they have much longer lifetimes. LISA will look for close white-dwarf binaries in our galaxy, and will 
probably see thousands of them. White dwarfs are not as compact as black holes or neutron stars. Although their masses can be similar to that of a neutron star their sizes are much larger, typically $3,000 \mathrm{~km}$ in radius. As a result, white-dwarf binaries never reach the last stable orbit, which would occur at roughly $1.5 \mathrm{kHz}$ for these masses. We will discuss the implications of multi-messenger astronomy for white-dwarf binaries in Section 7.4.

The maximum amplitude of the radiation from a white-dwarf binary will be several orders of magnitude smaller than that of a neutron star or black hole binary at the same distance but close to coalescence. However, a binary system with a short period is long lived, so the effective amplitude (after matched filtering) improves as the square root of the observing time. Besides that, these sources are nearer: there are many thousands of such systems in our galaxy radiating in the LISA frequency window above about $1 \mathrm{mHz}$, and LISA should be able to see all of them. Below $1 \mathrm{mHz}$ there are even more sources, so many that LISA will not resolve them individually, but will see them blended together in a stochastic background of radiation, as shown in Figure 5.

\subsubsection{Supermassive black hole binaries}

Observations indicate that the center of every galaxy probably hosts a black hole whose mass is in the range of $10^{6}-10^{9} M_{\odot}$ [305], with the black holes mass correlating well with the mass of the galactic bulge. A black hole whose mass is in the above range is called a supermassive black hole $(\mathrm{SMBH})$. There is now abundant observational evidence that galaxies often collide and merge, and there are good reasons to believe that when that happens, friction between the SMBHs and the stars and gas of the irregular merged galaxy will lead the SMBHs to spiral into a common nucleus and (on a timescale of some $10^{8} \mathrm{yr}$ ) even get close enough to be driven into complete orbital decay by gravitational radiation reaction. In many systems this should lead to a black hole merger within a Hubble time [221]. For a binary with two nonspinning $M=10^{6} M_{\odot}$ black holes, the frequency of emitted gravitational waves at the last stable orbit is, from Equation (29), $f_{\mathrm{LSO}}=4 \mathrm{mHz}$; during and after the merger the frequency rises from $4 \mathrm{mHz}$ to the quasi-normal-mode frequency of $24 \mathrm{mHz}$ (if the spin of the final black hole is negligible). (Naturally, all these frequencies simply scale inversely with the mass for other mass ranges.) This is in the frequency range of LISA, and observing these mergers is one of the central purposes of the mission.

SMBH mergers are so spectacularly strong that they will be visible in LISA's data stream even before applying any matched filter, although good models of the inspiral and particularly the merger radiation will be needed to extract source parameters. Because the masses of such black holes are so large, LISA can see essentially any merger that happens in its frequency band anywhere in the universe, even out to extremely high redshifts. It can thereby address astrophysical questions about the origin, growth and population of SMBHs. The recent discovery of an SMBH binary [221] and the association of X-shaped radio lobes with the merger of SMBH binaries [254] has further raised the optimism concerning SMBH merger rates, as has the suggestion that an SMBH has been observed to have been expelled from the center of its galaxy, an event that could only have happened as a result of a merger between two SMBHs [222]. The rate at which galaxies merge is about $1 \mathrm{yr}^{-1}$ out to a red-shift of $z=5$ [185], and LISA's detection rate for SMBH mergers might be roughly the same.

Modelling of the merger of two black holes requires numerical relativity, and the accuracy and reliability of numerical simulations is now becoming good enough that they will soon become an integral part of gravitational wave searches.

\subsubsection{Extreme and intermediate mass-ratio inspiral sources}

The SMBH environment of our own galaxy is known to contain a large number of compact objects and white dwarfs. Near the central SMBH there is a disproportionately large number of stellarmass black holes, which have sunk there through random gravitational encounters with the normal

Living Reviews in Relativity

http: //www. livingreviews.org/lrr-2009-2 
stellar population (dynamical friction). Three body interaction will occasionally drive one of these compact objects into a capture orbit of the central SMBH. The compact object will sometimes be captured $[305,338,337]$ into a highly eccentric trajectory $(e>0.99)$ with the periastron close to the last stable orbit of the SMBH. Since the mass of the captured object will be about $1-100 M_{\odot}$, while the SMBH will have a far greater mass, we essentially have a "test mass" falling in the geometry of a Kerr black hole. By Equation (33) we would expect that the small body would spend many orbits in the relativistic regime near the horizon of the large black hole: a $10 M_{\odot}$ black hole falling into a $10^{6} M_{\odot}$ black hole would require on the order of $10^{5}$ orbits. The emitted gravitational radiation $[317,179,178,67,171,57]$ would consist of a very long wave train that carries information about the nearly geodesic trajectory of the test body, thereby providing a very clean probe to survey the spacetime geometry of the central object (which could be a Kerr black hole or some other compact object) and test whether or not this geometry is as predicted by general relativity $[318,198,177,176,68]$.

This kind of event happens occasionally to every SMBH that lives in the center of a galaxy. Indeed, since the SNR from matched filtering builds up in proportion to the square root of the observation time $t_{\text {chirp }} \propto \nu^{-1}=(\mu / M)^{-1}$ [cf. Equation (33)] and the inherent amplitude of the radiation is linear in $\nu$ [cf. Equation (30)], the SNR varies with the symmetric mass ratio as $\sqrt{\nu}$ and typical SNR will be about ten to a thousand times smaller than an SMBH binary at the same distance. LISA will, therefore, be able to see such sources only to much smaller distances, say between 1 to 10 Gpc depending on the mass ratio. For events at such distances LISA's SNR after matched filtering could be in the range 10-100, but matched filtering will be very difficult because of the complexity of the orbit, especially of its evolution due to radiation effects. However, this volume of space contains a large number of galaxies, and the event rate is expected to be several tens to hundreds per year [67]. There will be a background from more distant sources that might in the end set the limit on how much sensitivity LISA has to these events.

Due to relativistic frame dragging, for each passage of the apastron the test body could execute several nearly circular orbits at its periastron. Therefore, long periods of low-frequency, small-amplitude radiation will be followed by several cycles of high-frequency, large-amplitude radiation $[317,179,178,67,171,57]$. The apastron slowly shrinks, while the periastron remains more or less at the same location, until the final plunge of the compact object before merger. Moreover, if the central black hole has a large spin then spin-orbit coupling leads to precession of the orbital plane thereby changing the polarization of the wave as seen by LISA.

Thus, there is a lot of structure in the waveforms owing to a number of different physical effects: contribution from higher-order multipoles due to an eccentric orbit, precession of the orbital plane, precession of the periastron, etc., and gravitational radiation backreaction plays a pivotal role in the dynamics of these systems. If one looks at the time-frequency map of such a signal one notices that the signal power is greatly smeared across the map [320], as compared to that of a sharp chirp from a nonspinning black-hole binary. For this reason, this spin modulated chirp is sometimes referred to as a smirch [322]. More commonly, such sources are called extreme mass ratio inspirals (EMRIs) and represent systems whose mass ratio is in the range of $\sim 10^{-3}-10^{-6}$. Inspirals of systems with their mass ratio in the range $\sim 10^{-2}-10^{-3}$ are termed intermediate mass ratio inspirals or IMRIs. These latter systems correspond to the inspiral of intermediate mass black holes of mass $\sim 10^{3}-10^{4} M_{\odot}$ and might constitute a prominent source in LISA provided the central SMBH grew in mass as a result of a number of mergers of small black holes [30, 31, 32].

While black hole perturbation theory with a careful treatment of radiation reaction is necessary for the description of EMRIs, IMRIs may be amenable to a description using a hybrid scheme of post-Newtonian approximations and perturbation theory. This is an area that requires more study. 


\subsection{Quasi-normal modes of a black hole}

In 1970, Vishveshwara [381] discussed a gedanken experiment, similar in philosophy to Rutherford's (real) experiment with the atom. In Vishveshwara's experiment, he scattered gravitational radiation off a black hole to explore its properties. With the aid of such a gedanken experiment, he demonstrated for the first time that gravitational waves scattered off a black hole will have a characteristic waveform, when the incident wave has frequencies beyond a certain value, depending on the size of the black hole. It was soon realized that perturbed black holes have quasi-normal modes (QNMs) of vibration and in the process emit gravitational radiation, whose amplitude, frequency and damping time are characteristic of its mass and angular momentum [296, 220]. We will discuss in Section 6.4 how observations of QNMs could be used in testing strong field predictions of general relativity.

We can easily estimate the amplitude of gravitational waves emitted when a black hole forms at a distance $r$ from Earth as a result of the coalescence of compact objects in a binary. The effective amplitude is given by Equation (20), which involves the energy $E$ put into gravitational waves and the frequency $f$ at which the waves come off. By dimensional arguments $E$ is proportional to the total mass $M$ of the resulting black hole. The efficiency at which the energy is converted into radiation depends on the symmetric mass ratio $\nu$ of the merging objects. One does not know the fraction of the total mass emitted nor the exact dependence on $\nu$. Flanagan and Hughes [164] argue that $E \sim 0.03(4 \nu)^{2} M$. The frequency $f$ is inversely proportional to $M$; indeed, for Schwarzschild black holes $f=(2 \pi M)^{-1}$. Thus, the formula for the effective amplitude takes the form

$$
h_{\mathrm{eff}} \sim \frac{4 \alpha \nu M}{\pi r}
$$

where $\alpha$ is a number that depends on the (dimensionless) angular momentum $a$ of the black hole and has a value between 0.7 (for $a=0$, Schwarzschild black hole) and 0.4 (for $a=1$, maximally spinning Kerr black hole). For stellar mass black holes at a distance of $200 \mathrm{Mpc}$ the amplitude is:

$$
h_{\mathrm{eff}} \simeq 10^{-21}\left(\frac{\nu}{0.25}\right)\left(\frac{M}{20 M_{\odot}}\right)\left(\frac{r}{200 \mathrm{Mpc}}\right)^{-1} .
$$

For SMBHs, even at cosmological distances, the amplitude of quasinormal mode signals is pretty large:

$$
h_{\mathrm{eff}} \simeq 3 \times 10^{-17}\left(\frac{\nu}{0.25}\right)\left(\frac{M}{2 \times 10^{6} M_{\odot}}\right)\left(\frac{r}{6.5 \mathrm{Gpc}}\right)^{-1} .
$$

In the first case we have a pair of $10 M_{\odot}$ black holes inspiraling and merging to form a single black hole. In this case the waves come off at a frequency of around $500 \mathrm{~Hz}$ [cf. Equation (13)]. The initial ground-based network of detectors might be able to pick these waves up by matched filtering, especially when an inspiral event precedes the ringdown signal. A $100 M_{\odot}$ black hole plunging into a $10^{6} M_{\odot}$ black hole at a distance of $6.5 \mathrm{Gpc}(z \simeq 1)$ gives out radiation at a frequency of about $15 \mathrm{mHz}$. Note that in the latter case the radiation is redshifted from $30 \mathrm{mHz}$ to $15 \mathrm{mHz}$. Such an event produces an amplitude just large enough to be detected by LISA. At the same distance, a pair of $10^{6} M_{\odot}$ SMBHs spiral in and merge to produce a fantastic amplitude of $3 \times 10^{-17}$, way above the LISA background noise. In this case, the signals would be given off at about $7.5 \mathrm{mHz}$ and will be loud and clear to LISA. It will not only be possible to detect these events, but also to accurately measure the masses and spins of the objects before and after merger and thereby test the black hole no-hair theorem and confirm whether the result of the merger is indeed a black hole or some other exotic object (e.g., a boson star or a naked singularity).

Living Reviews in Relativity

http://www. livingreviews.org//rr-2009-2 


\subsection{Stochastic background}

In addition to radiation from discrete sources, the universe should have a random gravitational wave field that results from a superposition of countless discrete systems and also from fundamental processes, such as the Big Bang. Observing any of these backgrounds would bring useful information, but the ultimate goal of detector development is the observation of the background radiation from the Big Bang. It is expected to be very weak, but it will come to us unhindered from as early as $10^{-30} \mathrm{~s}$, and it could illuminate the nature of the laws of physics at energies far higher than we can hope to reach in the laboratory.

It is usual to characterize the intensity of a random field of gravitational waves by its energy density as a function of frequency. Since the energy density of a plane wave is the same as its flux (when $c=1$ ), we have from Equation (17)

$$
\rho_{\mathrm{gw}}=\frac{\pi}{4} f^{2} h^{2} .
$$

But the wave field in this case is a random variable, so we must replace $h^{2}$ by a statistical mean square amplitude per unit frequency (Fourier transform power per unit frequency) called $S_{\mathrm{gw}}(f)$, so that the energy density per unit frequency is proportional to $f^{2} S_{\mathrm{gw}}(f)$. It is then conventional to talk about the energy density per unit logarithm of the frequency, which means multiplying by $f$. The result, after being careful about averaging over all directions of the waves and all independent polarization components, is [27, 359]

$$
\frac{d \rho_{\mathrm{gw}}}{d \ln f}=4 \pi^{2} f^{3} S_{\mathrm{gw}}(f) .
$$

Finally, what is of most interest is the energy density as a fraction of the closure or critical cosmological density, given by the Hubble constant $H_{0}$ as $\rho_{c}=3 H_{0}^{2} / 8 \pi$. The resulting ratio is called $\Omega_{\mathrm{gw}}(f)$ :

$$
\Omega_{\mathrm{gw}}(f)=\frac{10 \pi^{2}}{3 H_{0}^{2}} f^{3} S_{\mathrm{gw}}(f) .
$$

The only tight constraint on $\Omega_{\mathrm{gw}}$ from non-gravitational-wave astronomy is that it must be smaller than $10^{-5}$, in order not to disturb the agreement between the standard Big Bang model of nucleosynthesis (of helium and other light elements) and observation. If the universe contains this much gravitational radiation today, then at the time of nucleosynthesis the (blue-shifted) energy density of this radiation would have been comparable to that of the photons and the three neutrino species. Although the radiation would not have participated in the nuclear reactions, its extra energy density would have required that the expansion rate of the universe at that time be significantly faster, in order to evolve into the universe we see today. In turn, this faster expansion would have provided less time for the nuclear reactions to "freeze out", altering the abundances from the values that are observed today [281, 346]. First-generation interferometers should be able to set direct limits on the cosmological background at around this level. Radiation in the lowerfrequency LISA band, from galactic and extra-galactic binaries, is expected to be much smaller than this bound.

Random radiation is indistinguishable from instrumental noise in a single detector, at least for short observing times. If the random field is produced by an anisotropically-distributed set of astrophysical sources (the binaries in our galaxy, for example) then over a year, as the detector changes its orientation, the noise from this background should rise and fall in a systematic way, allowing it to be identified. But this is a rather crude way of detecting the radiation, and a better way is to perform a cross-correlation between two detectors, if available.

In cross-correlation, which amounts to multiplying the outputs and integrating, the random signal in one detector essentially acts as a template for the signal in the other detector. If they 
match, then there will be a stronger-than-expected correlation. Notice that they can only match well if the wavelength of the gravitational waves is longer than the separation between the detectors: otherwise time delays for waves reaching one detector before the other degrade the match. The outcome is not like standard matched filtering, however, since the "filter" of the first detector has as much noise superimposed on its template as the other detector. As a result, the amplitude SNR of the correlated field grows only with observing time $T$ as $T^{1 / 4}$, rather than the square root growth that characterizes matched filtering [359]. 


\section{Gravitational Wave Detectors and Their Sensitivity}

Detectors of gravitational waves generally divide into two classes: beam detectors and resonant mass detectors. In beam detectors, gravitational waves interact with a beam of electromagnetic radiation, which is monitored in some way to register the passage of the wave. In resonant mass detectors, the gravitational wave transfers energy to a massive body, from which the resultant oscillations are observed.

Both classes include a variety of systems. The principal beam detectors are the large groundbased laser interferometers currently operating in several locations around the globe, such as the LIGO system in the USA. The ESA-NASA LISA mission aims to put a laser interferometer into space to detect milliHertz gravitational waves. But beam detectors do not need to involve interferometry: the radio beams transponded to interplanetary spacecraft can carry the signature of a passing gravitational wave, and this method has been used to search for low-frequency gravitational waves. And radio astronomers have for many years monitored the radio beams of distant pulsars for evidence of gravitational waves; new radio instrumentation is turning this into a powerful and promising method of looking for stochastic backgrounds and individual sources. And at ultra-low frequencies, gravitational waves in the early universe may have left their imprint on the polarization of the cosmic microwave background.

Resonant mass detectors were the first kind of detector built in the laboratory to detect gravitational waves: Joseph Weber [387] built two cylindrical aluminum bar detectors and attempted to find correlated disturbances that might have been caused by a passing impulsive gravitational wave. His claimed detections led to the construction of many other bar detectors of comparable or better sensitivity, which never verified his claims. Some of those detectors were not developed further, but others had their sensitivities improved by making them cryogenic, and today there are two ultra-cryogenic detectors in operation (see Section 4.1).

In the following, we will examine the principal detection methods that hold promise today and in the near future.

\subsection{Principles of the operation of resonant mass detectors}

A typical "bar" detector consists of a cylinder of aluminum with a length $\ell \sim 3 \mathrm{~m}$, a very narrow resonant frequency between $f \sim 500 \mathrm{~Hz}$ and $1.5 \mathrm{kHz}$, and a mass $M \sim 1000 \mathrm{~kg}$. A short gravitational wave burst with $h \sim 10^{-21}$ will make the bar vibrate with an amplitude

$$
\delta \ell_{g w} \sim h \ell \sim 10^{-21} \mathrm{~m}
$$

To measure this, one must fight against three main sources of noise.

1. Thermal noise. The original Weber bar operated at room temperature, but the most advanced detectors today, Nautilus [51] and Auriga [227], are ultra-cryogenic, operating at $T=100 \mathrm{mK}$. At this temperature the root mean square ( $\mathrm{rms}$ ) amplitude of vibration is

$$
\left\langle\delta \ell^{2}\right\rangle_{t h}^{1 / 2}=\left(\frac{k T}{4 \pi^{2} M f^{2}}\right)^{1 / 2} \sim 6 \times 10^{-18} \mathrm{~m} .
$$

This is far larger than the gravitational wave amplitude expected from astrophysical sources. But if the material has a high $Q$ (say, $10^{6}$ ) in its fundamental mode, then that changes its thermal amplitude of vibration in a random walk with very small steps, taking a time $Q / f \sim 1000$ s to change by the full amount. However, a gravitational wave burst will cause a change in $1 \mathrm{~ms}$. In $1 \mathrm{~ms}$, thermal noise will have random-walked to an expected amplitude 
change $(1000 \mathrm{~s} / 1 \mathrm{~ms})^{1 / 2}=Q^{1 / 2}$ times smaller, or (for these numbers)

$$
\left\langle\delta \ell^{2}\right\rangle_{t h: 1 \mathrm{~ms}}^{1 / 2}=\left(\frac{k T}{4 \pi^{2} M f^{2} Q}\right)^{1 / 2} \sim 6 \times 10^{-21} \mathrm{~m} .
$$

So ultra-cryogenic bars can approach the goal of detection near $h=10^{-20}$ despite thermal noise.

2. Sensor noise. A transducer converts the bar's mechanical energy into electrical energy, and an amplifier increases the electrical signal to record it. If sensing of the vibration could be done perfectly, then the detector would be broadband: both thermal impulses and gravitational wave forces are mechanical forces, and the ratio of their induced vibrations would be the same at all frequencies for a given applied impulsive force.

But sensing is not perfect: amplifiers introduce noise, and this makes small amplitudes harder to measure. The amplitudes of vibration are largest in the resonance band near $f$, so amplifier noise limits the detector sensitivity to gravitational wave frequencies near $f$. But if the noise is small, then the measurement bandwidth about $f$ can be much larger than the resonant bandwidth $f / Q$. Typical measurement bandwidths are $10 \mathrm{~Hz}$, about $10^{4}$ times larger than the resonant bandwidths, and $100 \mathrm{~Hz}$ is not out of the question [59].

3. Quantum noise. The zero-point vibrations of a bar with a frequency of $1 \mathrm{kHz}$ are

$$
\left\langle\delta \ell^{2}\right\rangle_{\text {quant }}^{1 / 2}=\left(\frac{\hbar}{2 \pi M f}\right)^{1 / 2} \sim 4 \times 10^{-21} \mathrm{~m} .
$$

This is comparable to the thermal limit over $1 \mathrm{~ms}$. So, as detectors improve their thermal limits, they run into the quantum limit, which must be breached before a signal at $10^{-21}$ can be seen with such a detector.

It is not impossible to do better than the quantum limit. The uncertainty principle only sets the limit above if a measurement tries to determine the excitation energy of the bar, or equivalently the phonon number. But one is not interested in the phonon number, except in so far as it allows one to determine the original gravitational wave amplitude. It is possible to define other observables that also respond to the gravitational wave and can be measured more accurately by squeezing their uncertainty at the expense of greater errors in their conjugate observable [110]. It is not yet clear whether squeezing will be viable for bar detectors, although squeezing is now an established technique in quantum optics and will soon be implemented in interferometric detectors (see below).

Reliable gravitational wave detection, whether with bars or with other detectors, requires coincidence observations, in which two or more detectors confirm each other's findings. The principal bar detector projects around the world formed the International Gravitational Event Collaboration (IGEC) [202] to arrange for long-duration coordinated observations and joint data analysis. A report in 2003 of an analysis of a long period of coincident observing over three years found no evidence of significant events [50]. The ALLEGRO bar [243] at Louisiana State University made joint data-taking runs with the nearby LIGO interferometer, setting an upper limit on the stochastic gravitational-wave background at around $900 \mathrm{~Hz}$ of $h_{100}^{2} \Omega_{g w}(f) \leq 0.53$ [17]. More recently, because funding for many of the bar detector projects has become more restricted, only two groups continue to operate bars at present (end of 2008): the Rome [367] and Auriga [227] groups. The latest observational results from IGEC may be found in [54].

It is clear from the above discussion that bars have great difficulty achieving the sensitivity goal of $10^{-21}$. This limitation was apparent even in the $1970 \mathrm{~s}$, and that motivated a number

Living Reviews in Relativity

http://www.livingreviews.org/lrr-2009-2 
of groups to explore the intrinsically wide-band technique of laser interferometry, leading to the projects described in Section 4.3.1 below. However, the excellent sensitivity of resonant detectors within their narrow bandwidths makes them suitable for specialized, high-frequency searches, including cross-correlation searches for stochastic backgrounds [119]. Therefore, novel and imaginative designs for resonant-mass detectors continue to be proposed. For example, it is possible to construct large spheres of a similar size ( 1 to $3 \mathrm{~m}$ diameter) to existing cylinders. This increases the mass of the detector and also improves its direction-sensing. One can in principle push to below $10^{-21}$ with spheres [117]. A spherical prototype called MiniGRAIL[234] has been operated in the Netherlands[181]. A similar prototype called the Schenberg detector[203] is being built in Brazil [21]. Nested cylinders or spheres, or masses designed to sense multiple modes of vibration may also provide a clever way to improve on bar sensitivities [86].

While these ideas have interesting potential, funding for them is at present (2008) very restricted, and the two remaining bar detectors are likely to be shut down in the near future, when the interferometers begin operating at sensitivities clearly better than $10^{-21}$.

\subsection{Principles of the operation of beam detectors}

Interferometers use laser light to measure changes in the difference between the lengths of two perpendicular (or nearly perpendicular) arms. Typically, the arm lengths respond differently to a given gravitational wave, so an interferometer is a natural instrument to measure gravitational waves. But other detectors also use electromagnetic radiation, for example, ranging to spacecraft in the solar system and even pulsar timing.

The basic equation we need is for the effect of a plane linear gravitational wave on a beam of light. Suppose the angle between the direction of the beam and the direction of the plane wave is $\theta$. We imagine a very simple experiment in which the light beam originates at a clock, whose proper time is called $t$, and is received by a clock, whose proper time is $t_{f}$. The beam and gravitational-wave travel directions determine a plane, and we denote the polarization component of the gravitational wave that acts in this plane by $h_{+}(t)$, as measured at the location of the originating clock. The proper distance between the clocks, in the absence of the wave, is $L$. If the originating clock puts timestamps onto the light beam, then the receiving clock can measure the rate of arrival of the timestamps. If there is no gravitational wave, and if the clocks are ideal, then the rate will be constant, which can be normalized to unity. The effect of the gravitational wave is to change the arrival rate as a function of the emission rate by

$$
\frac{d t_{f}}{d t}=1+\frac{1}{2}(1+\cos \theta)\left\{h_{+}[t+(1-\cos \theta) L]-h_{+}(t)\right\} .
$$

This is very simple: the beam of light leaves the emitter at the time when the gravitational wave of phase $t$ passes the emitter, and it reaches the receiver at the time when the gravitational wave of phase $t+(1-\cos \theta) L$ is passing the receiver. So in the plane wave case, only the amplitudes of the wave at the emitting and receiving events affect the time delay.

In order to use such an arrangement to detect gravitational waves, one needs two very stable clocks. The best clocks today are stable to a few parts in $10^{16}$ [40], which implies that the minimum amplitude of gravitational waves that could be detected by such a two-clock experiment is $h \sim 10^{-15}$. However, this equation is also fundamental to the detection of gravitational waves by pulsar timing, in which the originating 'clock' is a pulsar. By correlating many pulsar signals, one can beat down the single-pulsar noise. This is described below in Section 4.4.2.

An arrangement that uses only one clock is one that sends a beam out to a receiver, which then reflects or retransmits (transponds) the beam back to the sender. The sender has the clock, which measures variations in the round-trip time. This method has been used with interplanetary spacecraft, which has the advantage that the only clock is on the ground, which can be made more 
stable than one carried in a spacecraft (see Section 4.4.1). For the same arrangement as above, the return time $t_{\text {return }}$ varies at the rate

$$
\begin{aligned}
\frac{d t_{\text {return }}}{d t} & =1+\frac{1}{2}\left\{(1-\cos \theta) h_{+}(t+2 L)-(1+\cos \theta) h_{+}(t)\right. \\
& \left.+2 \cos \theta h_{+}[t+L(1-\cos \theta)]\right\} .
\end{aligned}
$$

This is known as the three-term relation, the third term being the wave strength at the time the beam returns back to the sender.

But the sensitivity of such a one-path system as a gravitational wave detector is still limited by the stability of the clock. For that reason, interferometers have become the most sensitive beam detectors: effectively one arm of the interferometer becomes the 'clock', or at least the time standard, that variations in the other arm are compared to. Of course, if both arms are affected by a gravitational wave in the same way, then the interferometer will not see the wave. But this happens only in very special geometries. For most wave arrival directions and polarizations, the arms are affected differently, and a simple interferometer measures the difference between the round-trip travel time variations in the two arms. For the triangular space array LISA, the measured signal is somewhat more complex (see Section 4.4 .3 below), but it still preserves the principle that the time reference for one arm is a combination of the others.

\subsubsection{The response of a ground-based interferometer}

Ground-based interferometers are the most sensitive detectors operating today, and are likely to make the first direct detections [197]. The largest detectors operating today are the LIGO detectors [302], two of which have arm lengths of $4 \mathrm{~km}$. This is much smaller than the wavelength of the gravitational wave, so the interaction of one arm with a gravitational wave can be well approximated by the small- $L$ approximation to Equation (43), namely

$$
\frac{d t_{\text {return }}}{d t}=1+\sin ^{2} \theta L \dot{h}_{+}(t) .
$$

(See [69] for first corrections to the short-arm approximation.) To analyze the full detector, where the second arm will normally point out of the plane we have been working in up till now, it is helpful to go over to a tensorial expression, independent of special coordinate orientations. The gravitational wave will act in the plane transverse to the propagation direction; let us call that direction $\hat{N}$ and let us set up radiation basis vectors $\hat{e}_{x}^{R}$ and $\hat{e}_{y}^{R}$ in the transverse plane, such that $\hat{e}_{x}^{R}$ lies in the plane formed by the wave propagation direction and the arm of our gravitational wave sensor, which lies along the $x$-axis of the detector plane, whose unit vector is $\hat{e}_{x}$. (For a picture of this geometry, see the left-hand panel of Figure 3, where for the moment we are ignoring the $y$-arm of the detector shown there.)

With these definitions, the wave amplitude $h_{+}$is the one that has $\hat{e}_{x}^{R}$ and $\hat{e}_{y}^{R}$ as the axes of its ellipse. The full wave amplitude is described, as in Equation (6), by the wave tensor

$$
\mathbf{h}(t)=h_{+}(t) \mathbf{e}_{+}+h_{\times}(t) \mathbf{e}_{\times},
$$

where $\mathbf{e}_{+}$and $\mathbf{e}_{\times}$are the polarization tensors associated with these basis vectors (compare Equation (4)):

$$
\mathbf{e}_{+}=\left(\hat{e}_{x}^{R} \otimes \hat{e}_{x}^{R}-\hat{e}_{y}^{R} \otimes \hat{e}_{y}^{R}\right), \quad \mathbf{e}_{\times}=\left(\hat{e}_{x}^{R} \otimes \hat{e}_{y}^{R}+\hat{e}_{y}^{R} \otimes \hat{e}_{x}^{R}\right) .
$$

The unique way of expressing Equation (44) in terms of $\mathbf{h}$ is

$$
\left(\frac{d t_{\text {return }}}{d t}\right)_{\mathrm{x}-\mathrm{arm}}=1+L \hat{e}_{x} \cdot \dot{\mathbf{h}} \cdot \hat{e}_{x} .
$$

Living Reviews in Relativity

http: //www . livingreviews . org/lrr-2009-2 
This does not depend on any special orientation of the arm relative to the wave direction, and does not depend on the basis we chose in the transverse plane, so we can use it as well for the second arm of the interferometer, no matter what its orientation. Let us assume it lies along the unit vector by $\hat{e}_{y}$. (We do not, in fact, have to assume that the two arms are perpendicular to each other, but it simplifies the diagram a little.) The return-time derivative along the second arm is then given by

$$
\left(\frac{d t_{\text {return }}}{d t}\right)_{\mathrm{x}-\mathrm{arm}}=1+L \hat{e}_{y} \cdot \dot{\mathbf{h}} \cdot \hat{e}_{y}
$$

. The interferometer responds to the difference between these times,

$$
\left(\frac{d \delta t_{\text {return }}}{d t}\right)=\left(\frac{d t_{\text {return }}}{d t}\right)_{\mathrm{x}-\mathrm{arm}}-\left(\frac{d t_{\mathrm{return}}}{d t}\right)_{\mathrm{y}-\mathrm{arm}}=L \hat{e}_{x} \cdot \dot{\mathbf{h}} \cdot \hat{e}_{x}-L \hat{e}_{y} \cdot \dot{\mathbf{h}} \cdot \hat{e}_{y}
$$

. By analogy with the wave tensor, we define the detector tensor $\mathbf{d}$ by [146]

$$
\mathbf{d}=L\left(\hat{e}_{x} \otimes \hat{e}_{x}-\hat{e}_{y} \otimes \hat{e}_{y}\right) .
$$

(If the arms are not perpendicular this expression would still give the correct tensor if the unit vectors lie along the actual arm directions.) Then we can express the differential return time rate in the simple invariant form

$$
\left(\frac{d \delta t_{\text {return }}}{d t}\right)=\mathbf{d}: \dot{\mathbf{h}}
$$

where the notation $\mathbf{d}: \mathbf{h} \equiv d_{l m} h^{l m}$ denotes the Euclidean scalar product of the tensors $\mathbf{d}$ and $\mathbf{h}$. Equation (49) can be integrated over time to give the instantaneous path-length (or time-delay, or phase) difference between the arms, as measured by the central observer's proper time clock:

$$
\delta t_{\text {return }}(t)=\mathbf{d}: \mathbf{h} .
$$

This is a robust and compact expression for the response of any interferometer to any wave in the long-wavelength (short-arm) limit. Its dependence on the wave direction is called its antenna pattern.

It is conventional to re-express this measurable in terms of the stretching of the arms of the interferometer. Within our approximation that the arms are shorter than a wavelength, this makes sense: it is possible to define a local inertial coordinate system that covers the entire interferometer, and within this coordinate patch (where light travels at speed 1) time differences measure proper length differences. The differential return time is twice the differential length change of the arms:

$$
\delta L(t)=\frac{1}{2} \mathbf{d}: \mathbf{h} .
$$

For a bar detector of length $L$ lying along the director $\hat{a}$, the detector tensor is

$$
\mathbf{d}=L \hat{a} \otimes \hat{a},
$$

although one must be careful that the change in proper length of a bar is not simply given by Equation (51), because of the restoring forces in the bar.

When dealing with observations by more than one detector, it is not convenient to tie the alignment of the basis vectors in the sky plane with those in the detector frame, as we have done in the left-hand panel of Figure 3, since the detectors will have different orientations. Instead it will usually be more convenient to choose polarization tensors in the sky plane according to some universal reference, e.g., using a convenient astronomical reference frame. The right-hand panel of 
Figure 3 shows the general situation, where the basis vectors $\hat{\alpha}$ and $\hat{\beta}$ are rotated by an angle $\psi$ from the basis used in the left-hand panel. The polarization tensors on this new basis,

$$
\epsilon_{+}=(\hat{\alpha} \otimes \hat{\alpha}-\hat{\beta} \otimes \hat{\beta}), \quad \epsilon_{\times}=(\hat{\alpha} \otimes \hat{\beta}+\hat{\beta} \otimes \hat{\alpha}),
$$

are found by the following transformation from the previous ones:

$$
\begin{aligned}
& \epsilon_{+}=\mathbf{e}_{+} \cos 2 \psi+\mathbf{e}_{\times} \sin 2 \psi, \\
& \epsilon_{\times}=-\mathbf{e}_{+} \sin 2 \psi+\mathbf{e}_{\times} \cos 2 \psi .
\end{aligned}
$$

Then one can write Equation (51) as

$$
\frac{\delta L(t)}{L}=F_{+}(\theta, \phi, \psi) h_{+}(t)+F_{\times}(\theta, \phi, \psi) h_{\times}(t),
$$

where $F_{+}$and $F_{\times}$are the antenna pattern functions for the two polarizations defined on the sky-plane basis by

$$
F_{+} \equiv \mathbf{d}: \mathbf{e}_{+}, \quad F_{\times} \equiv \mathbf{d}: \mathbf{e}_{\times} .
$$

Using the geometry in the right-hand panel of Figure 3, one can show that

$$
\begin{aligned}
& F_{+}=\frac{1}{2}\left(1+\cos ^{2} \theta\right) \cos 2 \phi \cos 2 \psi-\cos \theta \sin 2 \phi \sin 2 \psi, \\
& F_{\times}=\frac{1}{2}\left(1+\cos ^{2} \theta\right) \cos 2 \phi \sin 2 \psi+\cos \theta \sin 2 \phi \cos 2 \psi
\end{aligned}
$$
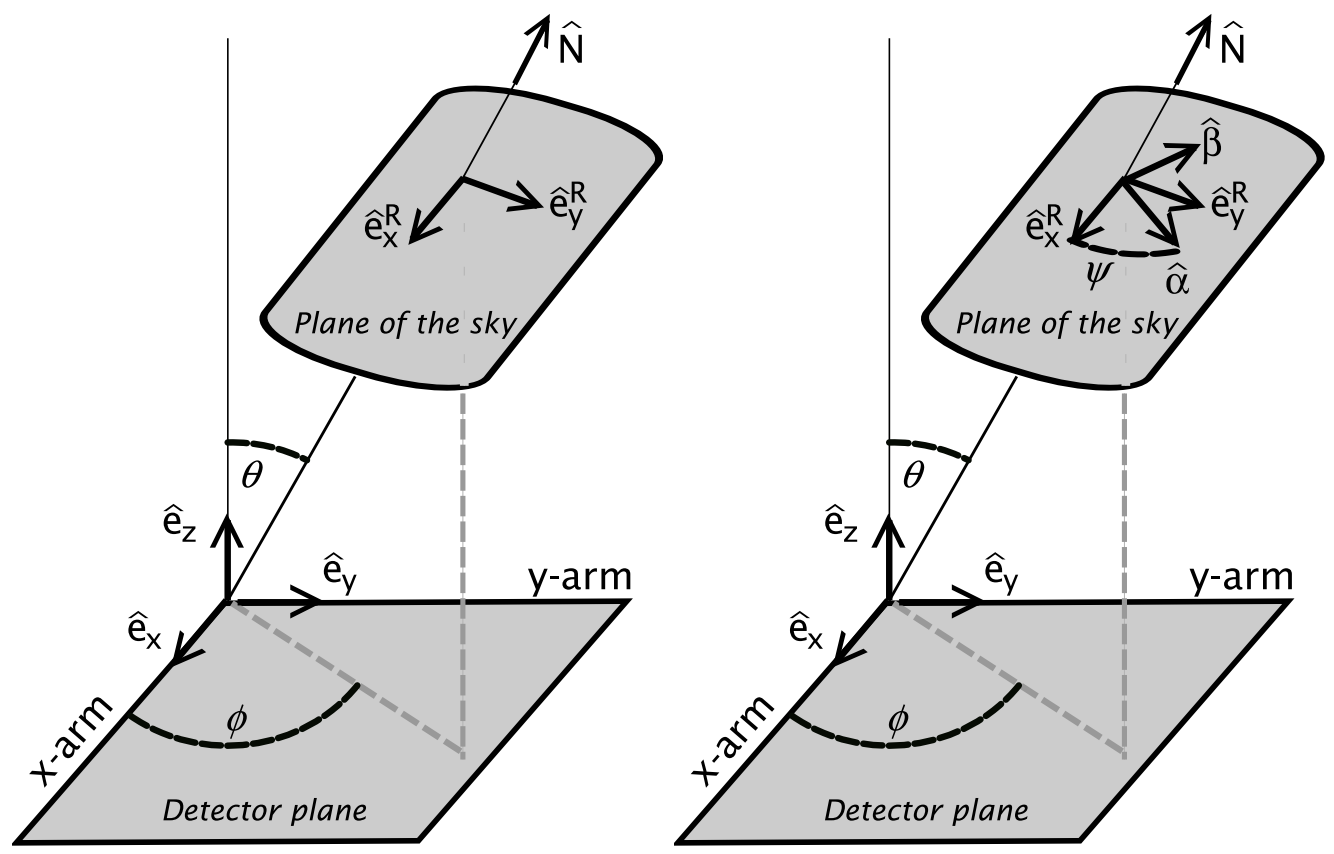

Figure 3: The relative orientation of the sky and detector frames (left panel) and the effect of a rotation by the angle $\psi$ in the sky frame (left panel).

These are the antenna-pattern response functions of the interferometer to the two polarizations of the wave as defined in the sky plane [359]. If one wants the antenna pattern referred to the 
detector's own axes, then one just sets $\psi=0$. If the arms of the interferometer are not perpendicular to each other, then one defines the detector-plane coordinates $x$ and $y$ in such a way that the bisector of the angle between the arms lies along the bisector of the angle between the coordinate axes [334]. Note that the maximum value of either $F_{+}$or $F_{\times}$is 1 .

The corresponding antenna-pattern functions of a bar detector whose longitudinal axis is aligned along the $z$ direction, are

$$
F_{+}=\sin ^{2} \theta \cos 2 \psi, \quad F_{\times}=\sin ^{2} \theta \sin 2 \psi .
$$

Any one detector cannot directly measure both independent polarizations of a gravitational wave at the same time, but responds rather to a linear combination of the two that depends on the geometry of the detector and source direction. If the wave lasts only a short time, then the responses of three widely-separated detectors, together with two independent differences in arrival times among them, are, in principle, sufficient to fully reconstruct the source location and gravitational wave polarization. A long-lived wave will change its location in the antenna pattern as the detector moves, and it will also be frequency modulated by the motion of the detector; these effects are in principle sufficient to determine the location of the source and the polarization of the wave.
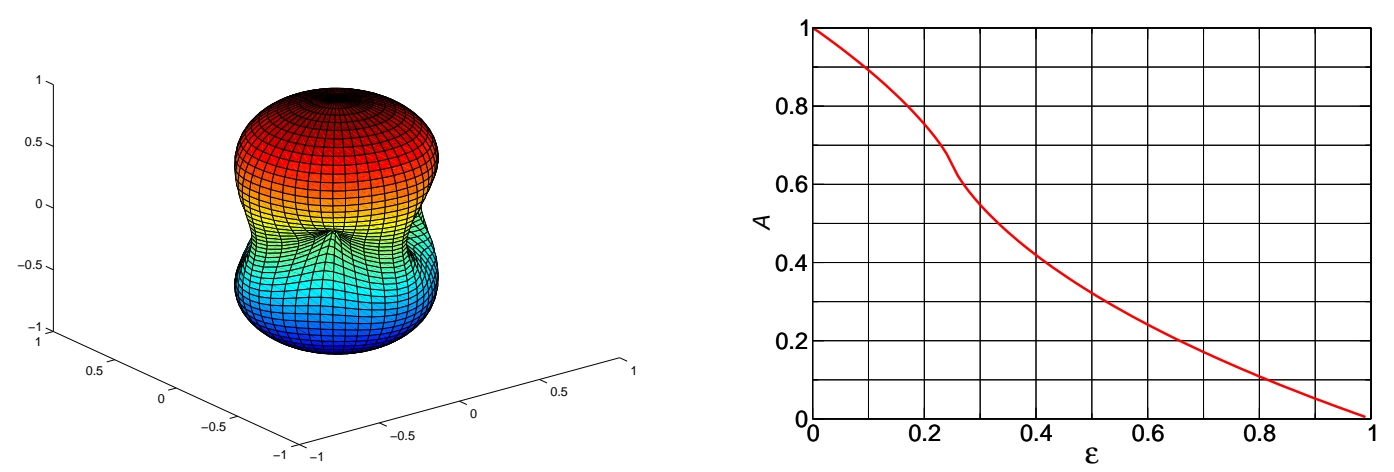

Figure 4: The antenna pattern of an interferometric detector (left panel) with the arms in the $x$ - $y$ plane and oriented along the two axes. The response $F$ for waves coming from a certain direction is proportional to the distance to the point on the antenna pattern in that direction. Also shown is the fractional area in the sky over which the response exceeds a fraction $\epsilon$ of the maximum (right panel).

Since the polarization angle of an incoming gravitational wave would generally be expected to be unrelated to its direction of arrival, depending rather on the internal orientations in the source, it is useful to characterize the directional sensitivity of a detector by averaging over the polarization angle $\psi$. If the wave has a given amplitude $h$ and is linearly polarized, then, if we are interested in a single detector's response, it is always possible to align the polarization angle $\psi$ in the sky plane with that of the wave, so that the wave has pure +-polarization. Then the rms response function of the detector is

$$
\bar{F}=\left(\int F_{+}^{2} \mathrm{~d} \psi\right)^{1 / 2} .
$$

The function $\bar{F}$ is often simply called the antenna pattern. For a resonant bar, the antenna pattern is

$$
\bar{F}=\sin ^{2} \theta,
$$

and for an interferometer, it is given by

$$
\bar{F}^{2}=\frac{1}{4}\left(1+\cos ^{2} \theta\right)^{2} \cos ^{2} 2 \phi+\cos ^{2} \theta \sin ^{2} 2 \phi .
$$


The antenna pattern of an interferometric detector is plotted in the left panel of Figure 4, which clearly shows the quadrupolar nature of the detector. Note that the response of an interferometer is the best for waves coming from a direction orthogonal to the plane containing the detector, and it is zero for waves in the plane of an interferometer's arms (i.e., $\theta=\pi / 2$ ) that arrive from a direction bisecting the two arms (i.e., $\phi=\pi / 4$ ) or from directions differing from this by a multiple of $\pi / 2$. What is the response of an antenna to a linearly-polarized source at a random location in the sky? This is given by the rms value of $\bar{F}$ over the sky,

$$
\left[\frac{1}{4 \pi} \int \bar{F}^{2} \sin \theta \mathrm{d} \theta \mathrm{d} \phi\right]^{1 / 2}
$$

which is smaller than the maximum response by a factor of $2 / \sqrt{15}(52 \%)$ for a bar detector and by $\sqrt{2 / 5}(63 \%)$ for an interferometer.

The polarization amplitudes of the radiation from an inspiraling binary, a rotating neutron star, or a ringing black hole, take a simple form as follows:

$$
h_{+}=\frac{h_{0}}{2}\left(1+\cos ^{2} \iota\right) \cos \Phi(t), \quad h_{\times}=h_{0} \cos \iota \sin \Phi(t),
$$

where $h_{0}$ is an overall (possibly time-dependent) amplitude, $\Phi(t)$ is the signal's phase and $\iota$ is the angle made by the characteristic direction in the source (e.g., the orbital or the spin angular momentum) with the line of sight. In this case, the response takes a particularly simple form:

$$
h(t)=F_{+} h_{+}+F_{\times} h_{\times}=A h_{0} \cos \left(\Phi(t)-\Phi_{0}\right),
$$

where

$$
A=\left(A_{+}^{2}+A_{\times}^{2}\right)^{1 / 2}, \quad \tan \Phi_{0}=\frac{A_{\times}}{A_{+}}, \quad A_{+}=\frac{1}{2} F_{+}\left(1+\cos \iota^{2}\right), \quad A_{\times}=F_{\times} \cos \iota .
$$

Note that $A$, just as $F$, takes values in the range $[0,1]$. In this case the average response has to be worked out by considering all possible sky locations, polarizations (which drops out of the calculation) and source orientations. More precisely, the rms response is

$$
\bar{A}=\frac{1}{8 \pi^{2}} \int_{0}^{\pi} \sin \iota \mathrm{d} \iota \int_{0}^{\pi} \sin \theta \mathrm{d} \theta \int_{0}^{2 \pi} \mathrm{d} \varphi\left(A_{+}^{2}+A_{\times}^{2}\right) .
$$

For an interferometer the above integral gives $2 / 5$. Thus, the rms response is still $40 \%$ of the peak response.

The right-hand panel of Figure 4 shows the percentage area of the sky over which the antenna pattern of an interferometric detector is larger than a certain fraction $\epsilon$ of the peak value. The response is better than the rms value over $40 \%$ of the sky, implying that gravitational wave detectors are fairly omni-directional. In comparison, the sky coverage of most conventional telescopes (radio, infrared, optical, etc.) is a tiny fraction of the area of the sky.

\subsection{Practical issues of ground-based interferometers}

A detector with an arm length of $4 \mathrm{~km}$ responds to a gravitational wave with an amplitude of $10^{-21}$ with

$$
\delta l_{g w} \sim h l \sim 4 \times 10^{-18} \mathrm{~m}
$$

Light takes only about $10^{-5} \mathrm{~s}$ to go up and down one arm, much less than the typical period of gravitational waves of interest. Therefore, it is beneficial to arrange for the light to remain in an 
arm longer than this, say for 100 round trips. This increases its effective path length by 100 and hence the shift in the position of a given phase of the light beam will be of order $10^{-16} \mathrm{~m}$. Most interferometers keep the light in the arms for this length of time by setting up optical cavities in the arms with low-transmissivity mirrors; these are called Fabry-Pérot cavities.

The main sources of noise against which a measurement must compete are:

1. Ground vibration. External mechanical vibrations must be screened out. These are a problem for bar detectors, too, but are more serious for interferometers, not least because interferometers bounce light back and forth between the mirrors, and so each reflection introduces further vibrational noise. Suspension/isolation systems are based on pendulums. A pendulum is a good mechanical filter for frequencies above its natural frequency. By hanging the mirrors on pendulums of perhaps $0.5 \mathrm{~m}$ length, one achieves filtering above a few Hertz. Since the spectrum of ground noise falls at higher frequencies, this provides suitable isolation. But these systems can be very sophisticated; the GEO600 [143] detector has a three-stage pendulum and other vibration isolation components [291]. The most ambitious multi-stage isolation system has been developed for the Virgo detector [175].

2. Thermal noise. Vibrations of the mirrors and of the suspending pendulum can mask gravitational waves. As with vibrational noise, this is increased by the bouncing of the light between the mirrors. Opposite to bars, interferometers perform measurements at frequencies far from the resonant frequency, where the amplitude of thermal vibration is largest. Thus, the pendulum suspensions have thermal noise at a few Hertz, so measurements will be made above $40 \mathrm{~Hz}$ in the first detectors. Internal vibrations of the mirrors have natural frequencies of several $\mathrm{kHz}$, which sets an effective upper limit to the observing band. By ensuring that both kinds of oscillations have very high $Q$, one can confine most of the vibration energy to a small bandwidth around the resonant frequency, so that at the measurement frequencies the vibration amplitudes are extremely small. This allows interferometers to operate at room temperature. But mechanical $Q \mathrm{~s}$ of $10^{7}$ or higher are required, and this is technically demanding.

Thermal effects produce other disturbances besides vibration. Some of the mirrors in interferometers are partly transmissive, as is the beam splitter. A small amount of light power is absorbed during transmission, which raises the temperature of the mirror and changes its index of refraction. The resulting "thermal lensing" can ruin the optical properties of the system, and random fluctuations in lensing caused by time-dependent thermal fluctuations (thermo-refractive noise) can appear at measurement frequencies. These effects can limit the amount of laser power that can be used in the detector. Other problems can arise from heating effects in the multiple-layer coatings that are applied to the reflective surfaces of mirrors.

3. Shot noise. The photons that are used to do interferometry are quantized, and so they arrive at random and make random fluctuations in the light intensity that can look like a gravitational wave signal. The more photons one uses, the smoother the interference signal will be. As a random process, the error improves with the square root of the number $N$ of photons. Using infrared light with a wavelength $\lambda \sim 1 \mu \mathrm{m}$, one can expect to measure to an accuracy of

$$
\delta l_{\text {shot }} \sim \lambda /(2 \pi \sqrt{N}) .
$$

To measure at a frequency $f$, one has to make at least $2 f$ measurements per second, so one can accumulate photons for a time $1 / 2 f$. With light power $P$, one gets $N=P /(h c / \lambda) /(2 f)$ photons. In order that $\delta l_{\text {shot }}$ should be below $10^{-16} \mathrm{~m}$, one needs high light power, far beyond the output of any continuous laser. 
Light-recycling techniques overcome this problem, by using light efficiently. An interferometer actually has two places where light leaves. One is where the interference is measured, the difference port. The other is the sum of the two return beams on the beam splitter, which goes back towards the input laser. Normally one makes sure that no light hits the interference sensor, so that only when a gravitational wave passes does a signal register there. This means that all the light normally returns toward the laser input, apart from small losses at the mirrors. Since mirrors are of good quality, only one part in $10^{3}$ or less of the light is lost during a $1 \mathrm{~ms}$ storage time. By placing a power-recycling mirror in front of the laser, one can reflect this wasted light back in, allowing power to build up in the arms until the laser merely resupplies the mirror losses [149]. This can dramatically reduce the power requirement for the laser. The first interferometers work with laser powers of 5-10 W. Upgrades will use ten or more times this input power.

4. Quantum effects. Shot noise is a quantum noise, and like all quantum noises there is a corresponding conjugate noise. As laser power is increased to reduce shot noise, the position sensing accuracy improves, and one eventually comes up against the Heisenberg uncertainty principle: the momentum transferred to the mirror by the measurement leads to a disturbance that can mask a gravitational wave. To reduce this backaction pressure fluctuation, scientists are experimenting with a variety of interferometer configurations that modify the quantum state of the light, by "squeezing" the Heisenberg uncertainty ellipse, in order to reduce the effect of this uncertainty on the variable being measured, at the expense of its (unmeasured) conjugate. The key point here is that we are using a quantum field (light) to measure an effectively classical quantity (gravitational wave amplitude), so we do not need to know everything about our quantum system: we just need to reduce the uncertainty in that part of the quantum field that responds to the gravitational wave at the readout of our interferometer. The best results on squeezing so far [371] have been obtained during preparations for the GEO-HF upgrade of the GEO600 detector [395]. These techniques may be needed for the second-generation advanced detectors and will certainly be needed for advances beyond that.

5. Gravity gradient noise. One noise that cannot be screened out is that due to changes in the local Newtonian gravitational field on the timescale of the measurements. A gravitational wave detector will respond to tidal forces from local sources just as well as to gravitational waves. Environmental noise comes not only from man-made sources, but even more importantly from natural ones: seismic waves are accompanied by changes in the gravitational field, and changes in air pressure are accompanied by changes in air density. The spectrum falls steeply with increasing frequency, so for first-generation interferometers this will not be a problem, but it may limit the performance of more advanced detectors. And it is the primary reason that detecting gravitational waves in the low-frequency band below $1 \mathrm{~Hz}$ must be done in space.

\subsubsection{Interferometers around the globe}

The two largest interferometer projects are LIGO [302] and VIRGO [175]. LIGO has built three detectors at two sites. At Hanford, Washington, there is a $4 \mathrm{~km}$ and a $2 \mathrm{~km}$ detector in the same vacuum system. At Livingston, Louisiana, there is a single $4 \mathrm{~km}$ detector, oriented to be as nearly parallel to the Hanford detector as possible. After a series of "engineering" runs, which helped to debug the instruments, interspersed with several "science runs", which helped to test and debug the data acquisition system and various analysis pipelines, LIGO reached its design sensitivity goal in the final months of 2005. In November 2005, LIGO began a two-year data-taking run, called S5, which acquired a year's worth of triple coincidence data among the three LIGO detectors. S5 ended on 30 September 2007. Although interferometers are pretty stable detectors, environmental

Living Reviews in Relativity

http://www.livingreviews.org/lrr-2009-2 
disturbances and instrumental malfunctions can cause them to lose lock during which the data quality will be either poor or ill defined. The typical duty cycle at one of the LIGO sites is about $80 \%$, and hence about two years of operation was required to accumulate a year's worth of triple coincident data. Up to date information on LIGO can be found on the project's website [103]. A recent review of LIGO's status is [303].

VIRGO finished commissioning its single 3-km detector at Cascina, near Pisa, in early 2007 and began taking data in coincidence with LIGO in May 2007, thus joining for the last part of S5. VIRGO is a collaboration among research laboratories in Italy and France, and its umbrella organization EGO looks after the operation of the site and planning for the future. There are websites for both VIRGO [380] and EGO [152]. A recent review of VIRGO's status is [20].

A smaller 600-m detector, GEO600, has been operational near Hanover, Germany, since 2001 [143]. It is a collaboration among research groups principally in Germany and Britain. Although smaller, GEO600 has developed and installed second-generation technology (primarily in its suspensions, mirror materials and interferometer configuration) that help it achieve a higher sensitivity. GEO600 technology is being transferred to LIGO and VIRGO as part of their planned upgrades, described below. Full information about GEO can be found on its website [261]. A recent review of GEO600's status is [396].

LIGO and GEO have worked together under the umbrella of the LIGO Scientific Collaboration (LSC) since the beginning of science data runs in 2001. The LSC contains dozens of groups from universities around the world, which contribute to data analysis and technology development. The two detector groups pool their data and analyze it jointly. The LSC has a website containing detailed information, and providing access to the publications and open-source software of the collaboration [236].

VIRGO has signed an agreement with the LSC to pool data and analyze it jointly, thereby creating a single worldwide network of long-baseline gravitational wave detectors. VIRGO is not, however, a member of the LSC.

The LSC has already published many papers on the analysis of data acquired during its science runs, and many more can be expected. The results from these science runs, which will be discussed later, are already becoming astrophysically interesting. The LSC maintains a public repository of its papers and contributions to conference proceedings [237].

For instance, although the search for continuous waves from known pulsars has not found any definitive candidates, it has been possible to set stringent upper limits $\epsilon \leq$ few $\times 10^{-6}$ on the magnitude of the ellipticity of some of these systems [10]. In particular, in the case of the Crab pulsar, gravitational wave observations have begun to improve [12] the upper limit on the strength of radiation obtained by radio observations of the spin-down rate.

A yet smaller detector in Japan, TAMA300 [362], with $300 \mathrm{~m}$ arms, was the first large-scale interferometer to achieve continuous operation, at a sensitivity of about $10^{-19}-10^{-20}$. TAMA is seen as a development prototype, and its sensitivity will be confined to higher frequencies (above $\sim 500 \mathrm{~Hz}$ ). An ambitious follow-on detector called the Large-scale Cryogenic Gravitational-Wave Telescope (LCGT) is being planned in Japan, and, as its name suggests, it will be the first to use cooled mirrors to reduce the effects of thermal noise. TAMA [269] and the LCGT [268] have websites where one can get more information. A recent review of TAMA's status is [130].

There are plans for a detector in Australia, and a small interferometer is operating in Western Australia [252]. The Australian Interferometric Gravitational Observatory (AIGO) [368] is a proposal of the Australian Consortium for Interferometric Gravitational Astronomy (ACIGA) [56]. The ACIGA collaboration is a member of the LSC and assists in mirror and interferometry development, but it is not yet clear whether and when a larger detector might be funded. From the point of view of extracting information from observations, it is very desirable to have large-scale detectors in Japan and Australia, because of their very long baselines to the USA and Europe. But the future funding of both LCGT and AIGO is not secure as of this writing (2008). 
The initial sensitivity levels achieved by LIGO, VIRGO, and GEO are just the starting point. Detailed plans exist for upgrades for all three projects. In October 2007, both LIGO and VIRGO began upgrading to enhanced detectors, which should improve on LIGO's S5 sensitivity by a factor of roughly two. These should come online in 2009. After a further observing run, called S6, the detectors will again shut down for a much more ambitious upgrade to advanced detectors, to operate around 2014. This will provide a further factor of five in sensitivity, and hence in range. Altogether the two upgrades will extend the volume of space that can be surveyed for gravitational waves by a factor of 1000, and this will make regular detections a virtual certainty. Advanced LIGO has a website giving the plans for the upgrade in the context of development from the initial sensitivity [235].

GEO600 will remain in science mode during the upgrade to enhanced detectors, just in case a nearby supernova or equally spectacular event should occur when the larger detectors are down. But, when the enhanced detectors begin operating, GEO will upgrade to GEO-HF [395], a modification designed to improve its sensitivity in the high-frequency region above $1 \mathrm{kHz}$, where its short arm length does not prevent it being competitive with the larger instruments. GEO is also a partner in the Advanced LIGO project, contributing high-power lasers and high-Q suspensions for controlling thermal noise.

Beyond that, scientists are now studying the technologies that may be needed for a further large step in sensitivity to third-generation detectors. This may involve cooling mirrors, using ultra-massive substrates of special materials, using purely nontransmissive optics, and even circumventing the quantum limit in interferometers, as has been studied for bars. The goal of thirdgeneration detectors would be to be limited just by gravity-gradient noise and quantum effects. A design study for a concept called the "Einstein Telescope" started in Europe in 2008.

\subsubsection{Very-high-frequency detectors}

The gravitational wave spectrum above the detection band of conventional interferometers, say above $10 \mathrm{kHz}$, may not be empty, and stochastic gravitational waves from the Big Bang may be present up to megaHertz frequencies and beyond. It is exceedingly difficult to build sensitive detectors at these high frequencies, but two projects have made prototypes: a microwave-based detector that senses the change in polarization as the electromagnetic waves follow a waveguide circuit as a gravitational wave passes by [126], and a more conventional light-based interferometer [23].

\subsection{Detection from space}

Space offers two important ingredients for beam detectors: long arms and a free vacuum. In this section, we describe the three ways that space has been and will be used for gravitational wave detection: ranging to spacecraft (Section 4.4.1), pulsar timing (Section 4.4.2), and direct detection using space-based interferometers (Section 4.4.3).

\subsubsection{Ranging to spacecraft}

Both NASA and ESA perform experiments in which they monitor the return time of communication signals with interplanetary spacecraft for the characteristic effect of gravitational waves. For missions to Jupiter and Saturn, for example, the return times are of order $2-4 \times 10^{3} \mathrm{~s}$. Any gravitational wave event shorter than this will, by Equation (43), appear three times in the time delay: once when the wave passes the Earth-based transmitter, once when it passes the spacecraft, and once when it passes the Earth-based receiver. Searches use a form of data analysis using pattern matching. Using two transmission frequencies and very stable atomic clocks, it is possible to achieve sensitivities for $h$ of order $10^{-13}$, and even $10^{-15}$ may soon be reached [40].

Living Reviews in Relativity

http: //www. livingreviews.org/lrr-2009-2 


\subsubsection{Pulsar timing}

Many pulsars, particularly the old millisecond pulsars, are extraordinarily regular clocks when averaged over timescales of a few years, with random timing irregularities too small for the best atomic clocks to measure. If one assumes that they emit pulses perfectly regularly, then one can use observations of timing irregularities of single pulsars to set upper limits on the background gravitational-wave field. Here, the one-way formula Equation (42) is appropriate. The transit time of a signal to the Earth from the pulsar may be thousands of years, so we cannot look for correlations between the two terms in a given signal. Instead, the delay is a combination of the effects of waves at the pulsar when the signal was emitted and waves at the Earth when it is received. If one observes a single pulsar, then because not enough is known about the intrinsic irregularity in pulse emission, the most one can do is to set upper limits on a background of gravitational radiation at very low frequencies [242, 344].

If one simultaneously observes two or more pulsars, then the Earth-based part of the delay is correlated, and this offers, in addition, a means of actually detecting strong gravitational waves with periods of several years that pass the Earth (in order to achieve the long-period stability of pulse arrival times). Observations are currently underway at a number of observatories. The most stringent limits to date are from the Parkes Pulsar Timing Array [208], which sets an upper limit on a stochastic background of $\Omega_{\mathrm{gw}} \leq 2 \times 10^{-8}$. Two further collaborations for timing have been formed: the European Pulsar Timing Array (EPTA) [345] and NanoGrav [39]. Astrophysical backgrounds in this frequency band are likely (see Section 8.2.2), so these arrays have a good chance of making early detections. Future timing experiments will be even more powerful, using new phased arrays of radio telescopes that can observe many pulsars simultaneously, such as the Low Frequency Array (LOFAR) [156] and the Square Kilometer Array [107].

Pulsar timing can also be used to search for individual events, not just a stochastic signal. The first example of an upper limit from such a search was the exclusion of a black-hole-binary model for 3C66B [209].

\subsubsection{Space interferometry}

Gravity-gradient noise on the Earth is much larger than the amplitude of any expected waves from astronomical sources at frequencies below about $1 \mathrm{~Hz}$, but this noise falls off rapidly as one moves away from the Earth. A detector in space would not notice the Earth's noisy environment. The Laser Interferometer Space Antenna (LISA) project, currently being developed in collaboration by ESA and NASA with a view toward launching in 2018, would open up the frequency window between $0.1 \mathrm{mHz}$ and $0.1 \mathrm{~Hz}$ for the first time [196, 144]. There are several websites that provide full information about this project [24, 153, 266].

We will see below that there are many exciting sources expected in this wave band, for example the coalescences of giant black holes in the centers of galaxies. LISA would see such events with extraordinary sensitivity, recording typical SNRs of 1000 or more for events at redshift one.

A space-based interferometer can have arm lengths much greater than a wavelength. LISA, for example, would have arms $5 \times 10^{6} \mathrm{~km}$ long, and that would be longer than half a wavelength for any gravitational waves above $30 \mathrm{mHz}$. In this regime, the response of each arm will follow the three-term formula we encountered earlier. The short-arm approximation we used for ground-based interferometers works for LISA only at the lowest frequencies in its observing band.

LISA will consist of three free-flying spacecraft, arranged in an array that orbits the sun at $1 \mathrm{AU}$, about 20 degrees behind the Earth in its orbit. They form an approximately equilateral triangle in a plane tilted at $60^{\circ}$ to the ecliptic, and their simple Newtonian elliptical orbits around the sun preserve this arrangement, with the array rotating backwards once per year as the spacecraft orbit the sun. By passing light along each of the arms, one can construct three different Michelsontype interferometers, one for each vertex. With this array one can measure the polarization of 
a gravitational wave directly. The spacecraft are too far apart to use simple mirrors to reflect light back along an arm: the reflected light would be too weak. Instead, LISA will have optical transponders: light from one spacecraft's on-board laser will be received at another, which will then send back light from its own laser locked exactly to the phase of the incoming signal.

The main environmental disturbances to LISA are forces from the sun: solar radiation pressure and pressure from the solar wind. To minimize these, LISA incorporates drag-free technology. Interferometry is referenced to an internal proof mass that falls freely, unattached to the spacecraft. The job of the spacecraft is to shield this mass from external disturbances. It does this by sensing the position of the mass and firing its own jets to keep itself (the spacecraft) stationary relative to the proof mass. To do this, it needs thrusters of very small thrust that have accurate control. The key technologies that have enabled the LISA mission are the availability of such thrusters, accelerometers needed to sense disturbances to the spacecraft, and lasers capable of continuously emitting $1 \mathrm{~W}$ of infrared light for many years. ESA is planning to launch a satellite called LISA Pathfinder to test all of these technologies in 2010 [230].

LISA is not the only proposal for an interferometer in space for gravitational wave detection. The DECIGO proposal is a more ambitious design, positioned at a higher frequency to fill the gap between LISA and ground-based detectors [213]. Even more ambitious, in the same frequency band, is the Big Bang Observer, a NASA concept study to examine what technology would be needed to reach the ultimate sensitivity of detecting a gravitational wave background from inflation at these frequencies [267].

\subsection{Characterizing the sensitivity of a gravitational wave antenna}

The performance of a gravitational wave detector is characterized by the power spectral density (henceforth denoted PSD) of its noise background. One can construct the noise PSD as follows; a gravitational wave detector outputs a dimensionless data train, say $x(t)$, which in the case of an interferometer is the relative strain in the two arms, scaled to represent the value of $h$ that would produce that strain if the wave is optimally oriented with respect to the detector. In the absence of any gravitational wave signal, the detector output is just an instance of noise $n(t)$, that is, $x(t)=n(t)$. The noise auto-correlation function $\kappa$ is defined as

$$
\kappa \equiv \overline{n\left(t_{1}\right) n\left(t_{2}\right)}
$$

where an overline indicates the average over an ensemble of noise realizations. In general, $\kappa$ depends both on $t_{1}$ and $t_{2}$. However, if the detector output is a stationary noise process, i.e., its performance is, statistically speaking, independent of time, then $\kappa$ depends only on $\tau \equiv\left|t_{1}-t_{2}\right|$.

The assumption of stationarity is not strictly valid in the case of real gravitational-wave detectors; however, if their performance doesn't vary greatly over time scales much larger than typical observation time scales, stationarity could be used as a working rule. While this may be good enough in the case of binary inspiral and coalescence searches, it is a matter of concern for the observation of continuous and stochastic gravitational waves. In this review, for simplicity, we shall assume that the detector noise is stationary. In this case the one-sided noise PSD, defined only at positive frequencies, is the Fourier transform of the noise auto-correlation function:

$$
\begin{aligned}
S_{h}(f) & \equiv \frac{1}{2} \int_{-\infty}^{\infty} \kappa(\tau) e^{2 \pi i f \tau} \mathrm{d} \tau, \quad f \geq 0 \\
& \equiv 0, \quad f<0
\end{aligned}
$$

where a factor of $1 / 2$ is included by convention. By using the Fourier transform of $n(t)$, that is $\tilde{n}(f) \equiv \int_{-\infty}^{\infty} n(t) e^{2 \pi i f t} \mathrm{~d} t$, in Equation (65) and substituting the resulting expression in Equa- 
tion (66), it is easy to shown that for a stationary noise process background

$$
\overline{\tilde{n}(f) \tilde{n}^{*}\left(f^{\prime}\right)}=\frac{1}{2} S_{h}(f) \delta\left(f-f^{\prime}\right),
$$

where $\tilde{n}^{*}(f)$ denotes the complex conjugate of $\tilde{n}(f)$. The above equation justifies the name PSD given to $S_{h}(f)$.

It is obvious that $S_{h}(f)$ has dimensions of time but it is conventional to use the dimensions of $\mathrm{Hz}^{-1}$, since it is a quantity defined in the frequency domain. The square root of $S_{h}(f)$ is the noise amplitude, $\sqrt{S_{h}(f)}$, and has dimensions of $\mathrm{Hz}^{-1 / 2}$. Both noise PSD and noise amplitude measure the noise in a linear frequency bin. It is often useful to define the power per logarithmic bin $h_{n}^{2}(f) \equiv f S_{h}(f)$, where $h_{n}(f)$ is called the effective gravitational-wave noise, and it is a dimensionless quantity. In gravitational-wave-interferometer literature one also comes across gravitational-wave displacement noise or gravitational-wave strain noise defined as $h_{\ell}(f) \equiv \ell h_{n}(f)$, and the corresponding noise spectrum $S_{\ell}(f) \equiv \ell^{2} S_{h}(f)$, where $\ell$ is the arm length of the interferometer. The displacement noise gives the smallest strain $\delta \ell / \ell$ in the arms of an interferometer that can be measured at a given frequency.
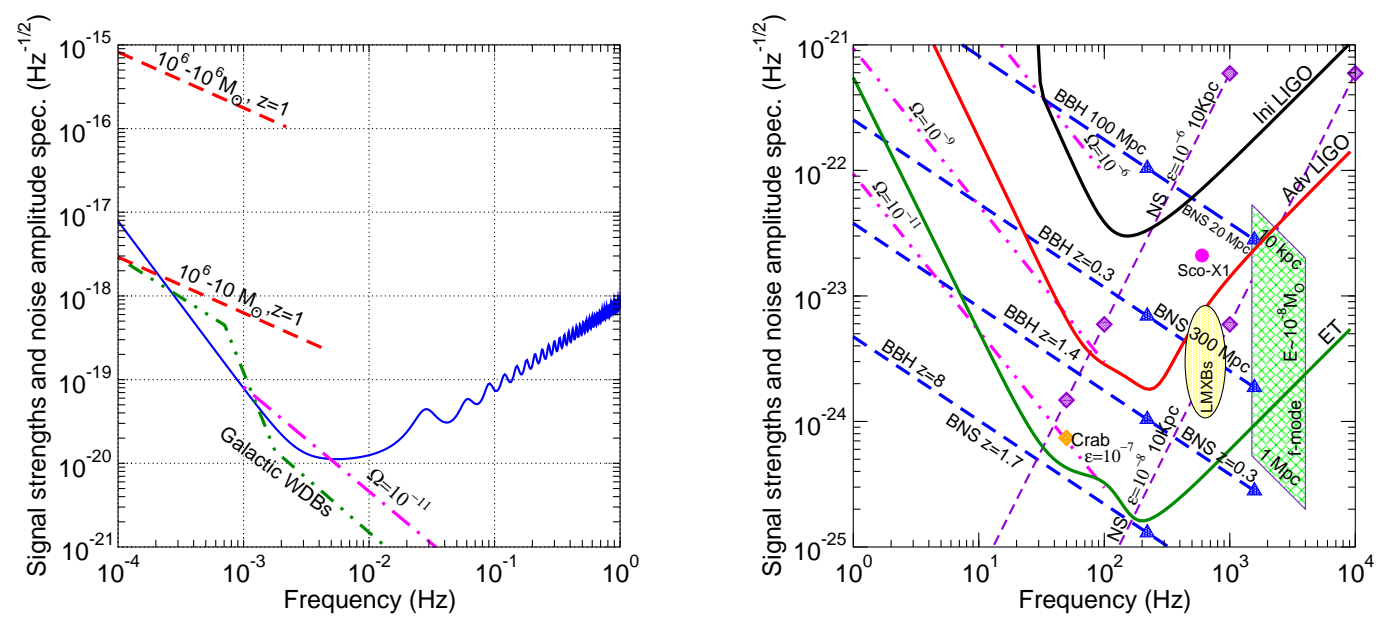

Figure 5: The right panel plots the noise amplitude spectrum, $\sqrt{f S_{h}(f)}$, in three generations of groundbased interferometers. For the sake of clarity, we have only plotted initial and advanced LIGO and a possible third generation detector sensitivities. VIRGO has similar sensitivity to LIGO at the initial and advanced stages, and may surpass it at lower frequencies. Also shown are the expected amplitude spectrum of various narrow and broad-band astrophysical sources. The left panel is the same as the right except for the LISA detector. The SMBH sources are assumed to lie at a redshift of $z=1$, but LISA can detect these sources with a good SNR practically anywhere in the universe. The curve labelled "Galactic WDBs" is the confusion background from the unresolvable Galactic population of white dwarf binaries.

\subsubsection{Noise power spectral density in interferometers}

As mentioned earlier, the performance of a gravitational wave detector is characterized by the one-sided noise PSD. The noise PSD plays an important role in signal analysis. In this review we will only discuss the PSDs of interferometric gravitational-wave detectors.

The sensitivity of ground based detectors is limited at frequencies less than a Hertz by the timevarying local gravitational field caused by a variety of different noise sources, e.g., low frequency seismic vibrations, density variation in the atmosphere due to winds, etc. Thus, for data analysis purposes, the noise PSD is assumed to be essentially infinite below a certain lower cutoff $f_{s}$. Above 
Table 1: Noise power spectral densities $S_{h}(f)$ of various interferometers in operation and under construction: GEO600, Initial LIGO (ILIGO), TAMA, VIRGO, Advanced LIGO (ALIGO), Einstein Telescope (ET) and LISA (instrumental noise only). For each detector the noise PSD is given in terms of a dimensionless frequency $x=f / f_{0}$ and rises steeply above a lower cutoff $f_{s}$. The parameters in the ET design sensitivity curve are $\alpha=-4.1, \beta=-0.69, a_{0}=186, b_{0}=233, b_{1}=31, b_{2}=-65, b_{3}=52, b_{4}=-42$, $b_{5}=10, b_{6}=12, c_{1}=14, c_{2}=-37, c_{3}=19, c_{4}=27$. (See also Figure 5.)

\begin{tabular}{ccccc}
\hline Detector & $f_{s} / \mathrm{Hz}$ & $f_{0} / \mathrm{Hz}$ & $S_{0} / \mathrm{Hz}^{-1}$ & $S_{h}(x) / S_{0}$ \\
& & & & \\
GEO & 40 & 150 & $1.0 \times 10^{-46}$ & $(3.4 x)^{-30}+34 x^{-1}+\frac{20\left(1-x^{2}+0.5 x^{4}\right)}{\left(1+0.5 x^{2}\right)}$ \\
ILIGO & 40 & 150 & $9.0 \times 10^{-46}$ & $(4.49 x)^{-56}+0.16 x^{-4.52}+0.52+0.32 x^{2}$ \\
TAMA & 75 & 400 & $7.5 \times 10^{-46}$ & $x^{-5}+13 x^{-1}+9\left(1+x^{2}\right)$ \\
VIRGO & 20 & 500 & $3.2 \times 10^{-46}$ & $(7.8 x)^{-5}+2 x^{-1}+0.63+x^{2}$ \\
ALIGO & 20 & 215 & $1.0 \times 10^{-49}$ & $x^{-4.14}-5 x^{-2}+\frac{111\left(1-x^{2}+0.5 x^{4}\right)}{1+0.5 x^{2}}$ \\
ET & 10 & 200 & $1.5 \times 10^{-52}$ & $x^{\alpha}+a_{0} x^{\beta}+\frac{b_{0}\left(1+b_{1} x+b_{2} x^{2}+b_{3} x^{3}+b_{4} x^{4}+b_{5} x^{5}+b_{6} x^{6}\right)}{1+c_{1} x+c_{2} x^{2}+c_{3} x^{3}+c_{4} x^{4}}$ \\
LISA & $10^{-5}$ & $10^{-3}$ & $9.2 \times 10^{-44}$ & $(x / 10)^{-4}+173+x^{2}$ \\
\hline
\end{tabular}

this cutoff, i.e., for $f \geq f_{s}$, Table 1 lists the noise PSD $S_{h}(f)$ for various interferometric detectors and some of these are plotted in Figure 5.

For LISA, Table 1 gives the internal instrumental noise only, taken from [162]. It is based on the noise budget obtained in the LISA Pre-Phase A Study [70]. However, in the frequency range $10^{-4}-10^{-2} \mathrm{~Hz}$, LISA will be affected by source confusion from astrophysical backgrounds produced by several populations of galactic binary systems, such as closed white-dwarf binaries, binaries consisting of Cataclysmic Variables, etc. At frequencies below about $1 \mathrm{mHz}$, there are too many binaries for LISA to resolve in, say, a 10-year mission, so that they form a Gaussian noise. Above this frequency range, there will still be many resolvable binaries which can, in principle, be removed from the data.

Nelemans et al. [272] estimate that the effective noise power contributed by binaries in the galaxy is

$$
S_{h}^{\text {gal }}=2.1 \times 10^{-38}\left(\frac{f}{f_{s}}\right)^{7 / 3} \quad \mathrm{~Hz}^{-1}, \quad f_{s}=10^{-3} \mathrm{~Hz}
$$

normalized to the same $f_{s}$ as we use for LISA in Table 1. This power is a mean frequency average based on projections of the population LISA will find, but, of course, above about $1 \mathrm{mHz}$, LISA will resolve many binaries and identify most of the members of this population. Barack and Cutler [67] have provided a prescription for including this effect when adding in the confusion noise. They make the conservative assumption that individual binaries contaminate the instrumental noise $S_{h}^{\text {instr }}$ (see Table 1) in such a way that, effectively, one or a few frequency resolution bins need to be cut out and ignored when detecting other signals, including, of course, other binary signals. This would have approximately the same effect as if the overall instrumental noise at that frequency were raised by an amount obtained simply by dividing the noise by the fraction $\eta$ of bins free of contamination. Of course, when this fraction reaches zero (below $1 \mathrm{mHz}$ ), this approximation is not valid, and instead one should just add the full binary confusion noise in Equation (68) to the 
instrumental noise. A smooth way of merging these two regimes is to set

$$
S_{h}^{\text {full }}=\min \left(\frac{1}{\eta} S_{h}^{\text {instr }}, \quad S_{h}^{\text {instr }}+S_{h}^{\text {gal }}\right),
$$

where $S_{h}^{\text {instr }}$ is from Table 1 and $S_{h}^{\text {gal }}$ is from Equation (68). This prescription uses the contaminated instrumental noise, when it is below the total noise power from the binaries, but then uses the total binary confusion power when the prescription for allowing for contamination breaks down.

The fraction $\eta$ of uncontaminated frequency bins as a function of frequency remains to be specified. Let $d N / d f$ be the number of binaries in the galaxy per unit frequency. Since the size of the frequency bin for an observation that lasts a time $T_{\text {obs }}$ is $1 / T_{\text {obs }}$, the expected number of binaries per frequency bin is

$$
\Delta N(f)=\frac{1}{T_{\text {obs }}} \frac{d N(f)}{d f} .
$$

Barack and Cutler multiply this by a "fudge factor" $\kappa>1$ to allow for the fact that any binary may contaminate several bins, so that $\kappa \Delta N(f)$ is the expected number of contaminated bins per binary. If this is small, then it will equal the fractional contamination at frequency $f$. In that case, the fraction of uncontaminated bins is just $1-\kappa \Delta N(f)$. However, if the expected contamination per bin approaches or exceeds one, then we have to allow for the fact that the binaries are really randomly distributed in frequency, so that the expected fraction not contaminated comes from the Poisson distribution,

$$
\eta=\exp (-\kappa \Delta N)
$$

Inserting this into Equation (69) gives a reasonable approximation to the effective instrumental noise if binaries cannot be removed in a clean way from the data stream when looking for other signals.

Because LISA will observe binaries for several years, the accuracy with which it will know the frequency, say, of a binary, will be much better than the frequency resolution of LISA during the observation of a transient source, such as many of the IMBH events considered by Barack and Cutler. Therefore, there is a good chance that, in the global LISA data analysis, the effective noise can be reduced below the one-year noise levels that are normally used in projecting the sensitivity of LISA and the science it can do.

\subsubsection{Sensitivity of interferometers in units of energy flux}

In radio astronomy one talks about the sensitivity of a telescope in terms of the limiting detectable energy flux from an astronomical source. We can do the same here too. Given the gravitational wave amplitude $h$ we can use Equation (17) to compute the flux of gravitational waves. One can translate the noise power spectrum $S_{h}(f)$, given in units of $\mathrm{Hz}^{-1}$ at frequency $f$, to Jy (Jansky), with the conversion factor $4 c^{3} f^{2} /(\pi G)$. In Figure 6 , the left panel shows the noise power spectrum in astronomical units of Jy and the right panel depicts the noise spectrum in units of $\mathrm{Hz}^{-1}$ together with lines of constant flux.

What is striking in Figure 6 is the magnitude of flux. While modern radio interferometers are sensitive to flux levels of milli and micro-Jy the gravitational wave interferometers need their sources to be $24-27$ orders of magnitude brighter. Turning this argument around, the gravitational wave sources we expect to observe are not really weak, but rather extremely bright sources. The difficulty in detecting them is due to the fact that gravitation is the weakest of all known interactions.

\subsection{Source amplitudes vs sensitivity}

How does one compare the gravitational wave amplitude of astronomical sources with the instrumental sensitivity and assess what sort of sources will be observable against noise? Comparisons 



Figure 6: The sensitivity of interferometers in terms of the limiting energy flux they can detect, Jy/Hz, (left panel) and in terms of the gravitational wave amplitude with lines of constant flux levels (right panel).

are almost always made in the frequency domain, since stationary noise is most conveniently characterized by its PSD.

The simplest signal to characterize is a long-lasting periodic signal with a given fixed frequency $f_{0}$. In an observation time T, all the signal power $\left|\tilde{h}\left(f_{0}\right)\right|^{2}$ is concentrated in a single frequency bin of width $1 / T$. The noise against which it competes is just the noise power in the same bin, $S_{h}\left(f_{0}\right) / T$. The power SNR is then $T\left|\tilde{h}\left(f_{0}\right)\right|^{2} / S_{h}\left(f_{0}\right)$, and the amplitude SNR is $\sqrt{T}\left|\tilde{h}\left(f_{0}\right)\right| /\left|S_{h}\left(f_{0}\right)\right|^{1 / 2}$. This improves with observation time as the square root of the time. The reason for this is that the noise is stationary, but longer and longer observation times permit the signal to compete only with noise in smaller and smaller frequency windows.

Of course, no expected gravitational-wave signal would have a single fixed frequency in the detector frame, because the detector is attached to the Earth, whose motion produces frequency modulations. But the principle of this SNR increase with time can still be maintained if one has a signal model that allows one to exclude more and more noise from competing with the signal over longer and longer periods of time. This happens with matched filtering, which we return to in Section 5.

Short-lived signals have wider bandwidths, and long observation times are not relevant. To characterize their SNR, it is useful to define the dimensionless noise power per logarithmic bandwidth, $f S_{h}(f)$, which we earlier called $h_{n}^{2}(f)$. The signal Fourier amplitude $\tilde{h}(f) \equiv \int_{-\infty}^{\infty} \mathrm{d} t h(t) e^{2 \pi i f t}$ has dimensions of $\mathrm{Hz}^{-1}$ and so the Fourier amplitude per logarithmic frequency, which is called the characteristic signal amplitude $h_{c}=f|\tilde{h}(f)|$, is dimensionless. This quantity should be compared with $h_{n}(f)$ to obtain a rough estimate of the SNR of the signal: SNR $\sim h_{c} / h_{n}$.

\subsection{Network detection}

Gravitational wave detectors are almost omni-directional. As discussed in Section 4.2.1, both interferometers and bars have good sensitivity over a large area of the sky. In this regard, gravitational wave antennas are unlike conventional astronomical telescopes, e.g., optical, radio, or infrared bands, which observe only a very small fraction of the sky at any given time. The good news is that gravitational wave interferometers will have good sky coverage and therefore only a small number (around six) are enough to survey the sky. The bad news, however, is that gravitational wave observations will not automatically provide the location of the source in the sky. It will either

Living Reviews in Relativity

http://www. livingreviews . org/Irr-2009-2 
be necessary to observe the same source in several non-co-located detectors and triangulate the position of the source using the information from the delay in the arrival times of the signals to different detectors, or observe for a long time and use the location-dependent Doppler modulation caused by the motion of the detector relative to the source to infer the source's position in the sky. The latter is a well-known technique in radio astronomy of synthesizing a long-baseline observation to gain resolution, and only possible for sources, such as rotating neutron stars or stochastic backgrounds, that last for a long enough duration.

A network of detectors is, therefore, essential for source reconstruction. Network observation is not only powerful in identifying a source in the sky, but independent observation of the same source in several detectors adds to the detection confidence, especially since the noise background in the first generation of interferometers is not well understood and is plagued by nonstationarity and non-Gaussianity.

\subsubsection{Coherent vs coincidence analysis}

The availability of a network of detectors offers two different methods by which the data can be combined. One can either first bring the data sets together, combine them in a certain way, and then apply the appropriate filter to the network data and integrate the signal coherently, coherent detection $[282,87,160,41]$, or first analyze the data from each detector separately by applying the relevant filters and then look for coincidences in the multi-dimensional space of intrinsic (masses of the component stars, their spins, ...) and extrinsic (arrival times, a constant phase, source location, ...) parameters, coincidence detection [205, 207, 160, 42, 353, 2, 6, 7, 8].

A recent comparison of coherent analysis vis-a-vis coincidence analysis under the assumption that the background noise is Gaussian and stationary has concluded that coherent analysis, as one might expect, is far better than coincidence analysis [263]. These authors also explore, to a limited extent, the effect of nonstationary noise and reach essentially the same conclusion.

At the outset, coherent analysis sounds like a good idea, since in a network of $N_{D}$ similar detectors the visibility of a signal improves by a factor of $\sqrt{N_{D}}$ over that of a single detector. One can take advantage of this enhancement in SNR to either lower the false alarm rate by increasing the detection threshold, while maintaining the same detection efficiency, or improve detection efficiency at a given false alarm rate.

However, there are two reasons that current data-analysis pipelines prefer coincidence analysis over coherent analysis. Firstly, since the detector noise is neither Gaussian nor stationary, coincidence analysis can potentially reduce the background rate far greater than one might think otherwise. Secondly, coherent analysis is computationally far more expensive than coincidence analysis and it is presently not practicable to employ coherent analysis.

Coincidence analysis is indeed a very powerful method to veto out spurious events. One can associate with each event in a given detector an ellipsoid, whose location and orientation depends on where in the parameter space and when the event was found, and the SNR can be used to fix the size of the ellipsoid [314]. One is associating with each event a 'sphere' of influence in the multi-dimensional space of masses, spins, arrival times, etc., and there is a stringent demand that the spheres associated with events from different detectors should overlap each other in order to claim a detection. Since random triggers from a network of detectors are less likely to be consistent with one another, this method serves as a very powerful veto.

It is probably not possible to infer beforehand which method might be more effective in detecting a source, as this might depend on the nature of the detector noise, on how the detection statistic is constructed, etc. An optimal approach might be a suitable combination of both of these methods. For instance, a coherent follow-up of a coincidence analysis (as is currently done by searches for compact binaries within the LSC) or to use coincidence criteria on candidate events from a coherent search. 
Coherent addition of data improves the visibility of the signal, but 'coherent subtraction' of the data in a detector network should lead to data products that are devoid of gravitational wave signals. This leads us naturally to the introduction of the null stream veto.

\subsubsection{Null stream veto}

Data from a network of detectors, when suitably shifted in time and combined linearly with coefficients that depend on the source location, will yield a time series that, in the ideal case, will be entirely be devoid of the gravitational signal. Such a combination is called a null stream. For instance, for a set of three misaligned detectors, each measuring a data stream $x_{k}(t), k=1,2,3$, the combination $x(t)=A_{23}(\theta, \varphi) h_{1}\left(t+\tau_{1}\right)+A_{31}(\theta, \varphi) h_{2}\left(t+\tau_{2}\right)+A_{12}(\theta, \varphi) h_{3}\left(t+\tau_{3}\right)$, where $A_{i j}$ are functions of the responses of the antennas $i$ and $j$, and $\tau_{k}$ 's, $k=1,2,3$, are time delays that depend on the source location and the location of the antenna, is a null stream. If $x_{k}(t), k=1,2,3$, contain a gravitational wave signal from an astronomical source, then $x(t)$ will not contain the signature of this source. In contrast, if $x(t)$ and $x_{k}(t)$ both contain the signature of a gravitational wave event, then that is an indication that one of the detectors has a glitch.

The existence and usefulness of a null stream was first pointed out by Gürsel and Tinto [184]. Wen and Schutz [390] proposed implementing it in LSC data analysis as a veto, and this has been taken up now by several search groups.

\subsubsection{Detection of stochastic signals by cross-correlation}

Stochastic background sources and their detection is discussed in more detail in Section 8. Here we will briefly mention the problem in the context of detector networks. As mentioned in Section 3.6, the universe might be filled with stochastic gravitational waves that were either generated in the primeval universe or by a population of background sources. For point sources, although each source in a population might not be individually detectable, they could collectively produce a confusion background via a random superposition of the waves from that population. Since the waves are random in nature, it is not possible to use the techniques described in Sections 4.7.1, 4.7.2 and 5.1 to detect a stochastic background. However, we might use the noisy stochastic signal in one of the detectors as a "matched-filter" for the data in another detector [359, 163, 27, 91]. In other words, it should be possible to detect a stochastic background by cross-correlating the data from a pair of detectors; the common gravitational-wave background will grow in time more rapidly than the random backgrounds in the two instruments, thereby facilitating the detection of the background.

If two instruments with identical spectral noise density $S_{h}$ are cross-correlated over a bandwidth $\Delta f$ for a total time $T$, the spectral noise density of the output is reduced by a factor of $(T \Delta f)^{1 / 2}$. Since the noise amplitude is proportional to the square root of $S_{h}$, the amplitude of a signal that can be detected by cross-correlation improves only with the fourth root of the observing time. This should be compared with the square root improvement that matched filtering gives.

The cross-correlation technique works well when the two detectors are situated close to one another. When separated, only those waves whose wavelength is larger than or comparable to the distance between the two detectors, or which arrive from a direction perpendicular to the separation between the detectors, can contribute coherently to the cross-correlation statistic. Since the instrumental noise builds up rapidly at lower frequencies, detectors that are farther apart are less useful in cross-correlation. However, very near-by detectors (as in the case of two LIGO detectors within the same vacuum tube in Hanford) will suffer from common background noise from the control system and the environment, making it rather difficult to ascertain if any excess noise is due to a stochastic background of gravitational waves.

Living Reviews in Relativity

http: //www . livingreviews .org//rr-2009-2 


\subsection{False alarms, detection threshold and coincident observation}

Gravitational-wave event rates in initial interferometers is expected to be rather low: about a few per year. Therefore, one has to set a high threshold, so that the noise-generated false alarms mimicking an event are negligible.

For a detector output sampled at $1 \mathrm{kHz}$ and processed through a large number of filters, say $10^{3}$, one has $\sim 3 \times 10^{13}$ instances of noise in a year. If the filtered noise is Gaussian, then the probability $P(x)$ of observing an amplitude in the range of $x$ to $x+d x$ is

$$
P(x) d x=\frac{1}{\sqrt{2 \pi} \sigma} \exp \left(\frac{-x^{2}}{2 \sigma^{2}}\right) d x,
$$

where $\sigma$ is the standard deviation. The above probability-distribution function implies that the probability that the noise amplitude is greater than a given threshold $\eta$ is

$$
P(x \mid x \geq \eta)=\int_{\eta}^{\infty} P(x) \mathrm{d} x=\frac{1}{\sqrt{2 \pi} \sigma} \int_{\eta}^{\infty} \exp \left(\frac{-x^{2}}{2 \sigma^{2}}\right) \mathrm{d} x .
$$

Demanding that no more than one noise-generated false alarm occur in a year's observation means that $P(x \mid x \geq \eta)=1 /\left(3 \times 10^{13}\right)$. Solving this equation for $\eta$, one finds that $\eta \simeq 7.5 \sigma$ in order that false alarms are negligible in a year's observation. Therefore, a source is detectable only if its amplitude is significantly larger than the effective noise amplitude, i.e., $f \tilde{h}(f) \gg h_{n}(f)$.

The reason for accepting only such high-sigma events is that the event rate of a transient source, i.e., a source lasting for a few seconds to minutes, such as a binary inspiral, could be as low as a few per year, and the noise generated false alarms, at low SNRs $\sim 3-4$, over a period of a year, tend to be quite large. Setting higher thresholds for detection helps in removing spurious, noise generated events. However, signal enhancement techniques (cf. Section 5) make it possible to detect a signal of relatively low amplitude, provided there are a large number of wave cycles and the shape of the wave is known accurately.

Real detector noise is neither Gaussian nor stationary and therefore the filtered noise cannot be expected to obey these properties either. One of the most challenging problems is how to remove or veto the false alarm generated by a non-Gaussian and/or nonstationary background. There has been some effort to address the issue of non-Gaussianity [124] and nonstationarity [260]; more work is needed in this direction. However, it is expected that the availability of a network of gravitational wave detectors alleviates the problem to some extent. This is because a high amplitude gravitational wave event will be coincidentally observed in several detectors, although not necessarily with the same SNR, while false alarms are, in general, not coincident, as they are normally produced by independent sources located close to the detectors.

We have seen that coincident observations help to reduce the false alarm rate significantly. The rate can be further reduced, and possibly even nullified, by subjecting coincident events to further consistency checks in a detector network consisting of four or more detectors. As discussed in Section 2, each gravitational wave event is characterized by five kinematic (or extrinsic) observables: location of the source with respect to the detector $(D, \theta, \varphi)$ and the two polarizations $\left(h_{+}, h_{\times}\right)$. Each detector in a network measures a single number, say the amplitude of the wave. In addition, in a network of $N$ detectors, there are $N-1$ independent time delays in the arrival times of the wave at various detector locations, giving a total of $2 N-1$ observables. Thus, the minimum number of detectors needed to reconstruct the wave and its source is $N=3$. More than three detectors in a network will have redundant information that will be consistent with the quantities inferred from any three detectors, provided the event is a true coincident event and not a chance coincidence, and most likely a true gravitational wave event. In a detector network consisting of $N(\geq 4)$ detectors, one can perform $2 N-6$ consistency checks. Such consistency checks further reduce the number of false alarms. 
When the shape of a signal is known, matched filtering is the optimal strategy to pull out a signal buried in Gaussian, stationary noise (see Section 5.1). The presence of high-amplitude transients in the data can render the background nonstationary and non-Gaussian, therefore matched filtering is not necessarily an optimal strategy. However, the knowledge of a signal's shape, especially when it has a broad bandwidth, can be used beyond matched filtering to construct a $\chi^{2}$ veto [28] to distinguish between triggers caused by a true signal from those caused by highamplitude transients or other artifacts. One specific implementation of the $\chi^{2}$ veto compares the expected signal spectrum with the real spectrum to quantify the confidence with which a trigger can be accepted to be caused by a true gravitational wave signal and has been the most powerful method for greatly reducing the false alarm rate. We shall discuss the $\chi^{2}$ veto in more detail in Section 5.1. 


\section{Data Analysis}

Observing gravitational waves requires a data analysis strategy, which is in many ways different from conventional astronomical data analysis. There are several reasons why this is so:

- Gravitational wave antennas are essentially omni-directional, with their response better than $50 \%$ of the root mean square over $75 \%$ of the sky (see Figure 4, right panel, recalling that the rms response is $2 / 5$ of the peak). Hence, data analysis systems will have to carry out all-sky searches for sources.

- Interferometers are typically broadband covering three to four orders of magnitude in frequency. While this is obviously to our advantage, as it helps to track sources whose frequency may change rapidly, it calls for searches to be carried out over a wide range of frequencies.

- In Einstein's theory, gravitational radiation has two independent states of polarization. Measuring polarization is of fundamental importance (as there are other theories of gravity in which the number of polarization states is more than two and in some theories even dipolar and scalar waves exist [392]) and has astrophysical implications too (for example, gravitational-wave-polarization measurement is one way to resolve the mass-inclination degeneracy of binary systems observed electromagnetically, as discussed in Section 7.1.1). Polarization measurements would be possible with a network of detectors, which means analysis algorithms that work with data from multiple antennas will have to be developed. This should also benefit coincidence analysis and the efficiency of event recognition.

- Unlike typical detection techniques for electromagnetic radiation from astronomical sources, most astrophysical gravitational waves are detected coherently, by following the phase of the radiation, rather than just the energy. That is, the SNR is built up by coherent superposition of many wave cycles emitted by a source. The phase evolution contains more information than the amplitude does and the signal structure is a rich source of the underlying physics. Nevertheless, tracking a signal's phase means searches will have to be made not only for specific sources but over a huge region of the parameter space for each source, placing severe demands both on the theoretical understanding of the emitted waveforms as well as on the data analysis hardware.

- Finally, gravitational wave detection is computationally intensive. Gravitational wave antennas acquire data continuously for many years at the rate of several megabytes per second. About $1 \%$ of this data is signal data; the rest is housekeeping data that monitors the operation of the detectors. The large parameter space mentioned above requires that the signal data be filtered many times for different searches, and this puts big demands on computing hardware and algorithms.

Data analysis for broadband detectors has been strongly developed since the mid 1980s [359, 331, 330]. The field has a regular series of annual Gravitational Wave Data Analysis Workshops; the published proceedings are a good place to find current thinking and challenges. Early coincidence experiments with interferometers [273] and bars [29] provided the first opportunities to apply these techniques. Although the theory is now fairly well understood [206], strategies for implementing data analysis depend on available computer resources, data volumes, astrophysical knowledge, and source modeling, and so are under constant revision.

We will begin with a discussion of the matched filtering algorithm and next use it to estimate the SNRs for binary coalescences in various detectors. After that, we will develop the theory of matched filtering further to work out the computational costs to carry out online searches, that is to search at the same rate as the data is acquired. In the final section, we will use the formalism 
developed in earlier sections to discuss parameter estimation. The foundations of signal analysis lie in the statistics of making "best estimates" of whether a signal is present in noisy data or not. See the Living Review by Jaranowski and Królak [206] for a discussion of this in the gravitational wave context.

\subsection{Matched filtering and optimal signal-to-noise ratio}

Matched filtering is a data analysis technique that efficiently searches for a signal of known shape buried in noisy data [186]. The technique consists in correlating the output of a detector with a waveform, variously known as a template or a filter. Given a signal $h(t)$ buried in noise $n(t)$, the task is to find an 'optimal' template $q(t)$ that would produce, on the average, the best possible SNR. In this review, we shall treat the problem of matched filtering as an operational exercise. However, this intuitive picture has a solid basis in the theory of hypothesis testing. The interested reader may consult any standard text book on signal analysis, for example Helstrom [186], for details.

Let us first fix our notation. We shall use $x(t)$ to denote the detector output, which is assumed to consist of a background noise $n(t)$ and a useful gravitational wave signal $h(t)$. The Fourier transform of a quantity $x(t)$ will be denoted $\tilde{x}(f)$ and is defined as

$$
\tilde{x}(f)=\int_{-\infty}^{\infty} x(t) e^{2 \pi i f t} \mathrm{~d} t
$$

\subsubsection{Optimal filter}

The detector output $x(t)$ is just a realization of noise $n(t)$, i.e., $x(t)=n(t)$, when no signal is present. In the presence of a signal $h(t)$ with an arrival time $t_{a}, x(t)$ takes the form,

$$
x(t)=h\left(t-t_{a}\right)+n(t) .
$$

The correlation $c$ of a template $q(t)$ with the detector output is defined as

$$
c(\tau) \equiv \int_{-\infty}^{\infty} x(t) q(t+\tau) \mathrm{d} t .
$$

In the above equation, $\tau$ is called the lag; it denotes the duration by which the filter function lags behind the detector output. The purpose of the above correlation integral is to concentrate all the signal energy at one place. The following analysis reveals how this is achieved; we shall work out the optimal filter $q(t)$ that maximizes the correlation $c(\tau)$ when a signal $h(t)$ is present in the detector output. To do this let us first write the correlation integral in the Fourier domain by substituting for $x(t)$ and $q(t)$, in the above integral, their Fourier transforms $\tilde{x}(f)$ and $\tilde{q}(f)$, i.e., $x(t) \equiv \int_{-\infty}^{\infty} \tilde{x}(f) \exp (-2 \pi i f t) \mathrm{d} f$ and $q(t) \equiv \int_{-\infty}^{\infty} \tilde{q}(t) \exp (-2 \pi i f t) \mathrm{d} f$, respectively. After some straightforward algebra, one obtains

$$
c(\tau)=\int_{-\infty}^{\infty} \tilde{x}(f) \tilde{q}^{*}(f) e^{-2 \pi i f \tau} \mathrm{d} f,
$$

where $\tilde{q}^{*}(f)$ denotes the complex conjugate of $\tilde{q}(f)$.

Since $n$ is a random process, $c$ is also a random process. Moreover, correlation is a linear operation and hence $c$ obeys the same probability distribution function as $n$. In particular, if $n$ is described by a Gaussian random process with zero mean, then $c$ is also described by a Gaussian distribution function, although its mean and variance will, in general, differ from those of $n$. The 
mean value of $c$, denoted by $S \equiv \bar{c}$, is, clearly, the correlation of the template $q$ with the signal $h$, since the mean value of $n$ is zero:

$$
S \equiv \bar{c}(\tau)=\int_{-\infty}^{\infty} \tilde{h}(f) \tilde{q}^{*}(f) e^{-2 \pi i f\left(\tau-t_{a}\right)} \mathrm{d} f .
$$

The variance of $c$, denoted $N^{2} \equiv \overline{(c-\bar{c})^{2}}$, turns out to be,

$$
N^{2}=\overline{(c-\bar{c})^{2}}=\int_{-\infty}^{\infty} S_{h}(f)|\tilde{q}(f)|^{2} \mathrm{~d} f .
$$

Now the SNR $\rho$ is defined by $\rho^{2} \equiv S^{2} / N^{2}$.

The form of integrals in Equations (77) and (78) leads naturally to the definition of the scalar product of waveforms. Given two functions, $a(t)$ and $b(t)$, we define their scalar product $\langle a, b\rangle$ to be $[159,161,114,128]$

$$
\langle a, b\rangle \equiv 2 \int_{0}^{\infty} \frac{\mathrm{d} f}{S_{h}(f)}\left[\tilde{a}(f) \tilde{b}^{*}(f)+\tilde{a}^{*}(f) \tilde{b}(f)\right] .
$$

Note that $S_{h}(f) \geq 0$ [cf. Equation (67)], consequently, the scalar product is real and positive definite.

Noting that the Fourier transform of a real function $h(t)$ obeys $\tilde{h}(-f)=\tilde{h}^{*}(f)$, we can write down the SNR in terms of the above scalar product:

$$
\rho^{2}=\frac{\left\langle h e^{2 \pi i f\left(\tau-t_{a}\right)}, S_{h} q\right\rangle}{\sqrt{\left\langle S_{h} q, S_{h} q\right\rangle}} .
$$

From this it is clear that the template $q$ that obtains the maximum value of $\rho$ is simply

$$
\tilde{q}(f)=\gamma \frac{\tilde{h}(f) e^{i 2 \pi f\left(\tau-t_{a}\right)}}{S_{h}(f)},
$$

where $\gamma$ is an arbitrary constant. From the above expression for an optimal filter we note two important things. First, the SNR is maximized when the lag parameter $\tau$ is equal to the time of arrival of the signal $t_{a}$. Second, the optimal filter is not just a copy of the signal, but rather it is weighted down by the noise PSD. We will see below why this should be so.

\subsubsection{Optimal signal-to-noise ratio}

We can now work out the optimal SNR by substituting Equation (81) for the optimal template in Equation (80),

$$
\rho_{\text {opt }}=\langle h, h\rangle^{1 / 2}=2\left[\int_{0}^{\infty} \mathrm{d} f \frac{|\tilde{h}(f)|^{2}}{S_{h}(f)}\right]^{1 / 2} .
$$

We note that the optimal SNR is not just the total energy of the signal (which would be $2 \int_{0}^{\infty} \mathrm{d} f|\tilde{h}(f)|^{2}$ ), but rather the integrated signal power weighted down by the noise PSD. This is in accordance with what we would guess intuitively: the contribution to the SNR from a frequency bin where the noise PSD is high is smaller than from a bin where the noise PSD is low. Thus, an optimal filter automatically takes into account the nature of the noise PSD.

The expression for the optimal SNR Equation (82) suggests how one may compare signal strengths with the noise performance of a detector. Note that one cannot directly compare $\widetilde{h}(f)$ 
with $S_{h}(f)$, as they have different physical dimensions. In gravitational wave literature one writes the optimal SNR in one of the following equivalent ways

$$
\rho_{\text {opt }}=2\left[\int_{0}^{\infty} \frac{\mathrm{d} f}{f} \frac{|\sqrt{f} \tilde{h}(f)|^{2}}{S_{h}(f)}\right]^{1 / 2}=2\left[\int_{0}^{\infty} \frac{\mathrm{d} f}{f} \frac{|f \tilde{h}(f)|^{2}}{f S_{h}(f)}\right]^{1 / 2}
$$

which facilitates the comparison of signal strengths with noise performance. One can compare the dimensionless quantities, $f|\tilde{h}(f)|$ and $\sqrt{f S_{h}(f)}$, or dimensionful quantities, $\sqrt{f}|\tilde{h}(f)|$ and $\sqrt{S_{h}(f)}$.

Signals of interest to us are characterized by several (a priori unknown) parameters, such as the masses of component stars in a binary, their intrinsic spins, etc., and an optimal filter must agree with both the signal shape and its parameters. A filter whose parameters are slightly mismatched with that of a signal can greatly degrade the SNR. For example, even a mismatch of one cycle in $10^{4}$ cycles can degrade the SNR by a factor two.

When the parameters of a filter and its shape are precisely matched with that of a signal, what is the improvement brought about, as opposed to the case when no knowledge of the signal is available? Matched filtering helps in enhancing the SNR in proportion to the square root of the number of signal cycles in the detector band, as opposed to the case in which the signal shape is unknown and all that can be done is to Fourier transform the detector output and compare the signal energy in a frequency bin to noise energy in that bin. We shall see below that, in initial interferometers, matched filtering leads to an enhancement of order 30-100 for compact binary inspiral signals.

\subsubsection{Practical applications of matched filtering}

Matched filtering is currently being applied to mainly two sources: detection of (1) chirping signals from compact binaries consisting of black holes and/or neutron stars and (2) continuous waves from rapidly-spinning neutron stars.

5.1.3.1 Coalescing binaries. In the case of chirping binaries, post-Newtonian theory (a perturbative approximation to Einstein's equations in which the relevant quantities are expanded as a power-series in $1 / c$, where $c$ is the speed of light) has been used to model the dynamics of these systems to a very high order in $v / c$, where $v$ is the relative speeds of the objects in the binary (see also Section 6.5, in which binaries are discussed in more detail). This is an approximation that can be effectively used to match filter the signal from binaries whose component bodies are of equal, or nearly equal, masses and the system is still "far" from coalescence. In reality, one takes the waveform to be valid until the last stable circular orbit (LSCO). In the case of binaries consisting of two neutron stars, or a neutron star and a black hole, tidal effects might affect the evolution significantly before reaching the LSCO. However, this is likely to occur at frequencies well-above the sensitivity band of the current ground-based detectors, so that for all practical purposes post-Newtonian waveforms are a good approximation to low-mass $\left(M<10 M_{\odot}\right)$ binaries.

As elucidated in Section 6.5.2, progress in analytical and numerical relativity has made it possible to have a set of waveforms for the merger phase of compact binaries too. The computational cost in matched filtering the merger phase, however, will not be high, as there will only be on the order of a few 100 cycles in this phase. But it is important to have the correct waveforms to enhance signal visibility and, more importantly, to enable strong-field tests of general relativity.

In the general case of black-hole-binary inspiral the search space is characterized by 17 different parameters. These are the two masses of the bodies, their spins, eccentricity of the orbit, its orientation at some fiducial time, the position of the binary in the sky and its distance from the Earth, the epoch of coalescence and phase of the signal at that epoch, and the polarization angle.

Living Reviews in Relativity

http: //www. livingreviews.org/lrr-2009-2 
However, not all these parameters are important in a matched filter search. Only those parameters that change the shape of the signal, such as the masses, orbital eccentricity and spins, or cause a modulation in the signal due to the motion of the detector relative to the source, such as the direction to the source, are to be searched for and others, such as the epoch of coalescence and the phase at that epoch, are simply reference points in the signal that can be determined without any significant burden on computational power.

For binaries consisting of nonspinning objects that are either observed for a short enough period that the detector motion can be neglected, or last for only a short time in the sensitive part of a detector's sensitivity band, there are essentially two search parameters - the component masses of the binary. It turns out that the signal manifold in this case is nearly flat, but the masses are curvilinear coordinates and are not good parameters for choosing templates. Chirp times, which are nonlinear functions of the masses, are very close to being Cartesian coordinates and template spacing is more or less uniform in terms of these parameters. Chirp times are post-Newtonian contributions at different orders to the duration of a signal starting from a time when the instantaneous gravitational-wave frequency has a fiducial value $f_{\mathrm{L}}$ to a time when the gravitational wave frequency formally diverges and system coalesces. For instance, the chirp times $\tau_{0}$ and $\tau_{3}$ at Newtonian and 1.5 PN orders, respectively, are

$$
\tau_{0}=\frac{5}{256 \pi \nu f_{\mathrm{L}}}\left(\pi M f_{\mathrm{L}}\right)^{-5 / 3}, \quad \tau_{3}=\frac{1}{8 \nu f_{\mathrm{L}}}\left(\pi M f_{\mathrm{L}}\right)^{-2 / 3},
$$

where $M$ is the total mass and $\nu=m_{1} m_{2} / M^{2}$ is the symmetric mass ratio. The above relations can be inverted to obtain $M$ and $\nu$ in terms of the chirp times:

$$
M=\frac{5}{32 \pi^{2} f_{\mathrm{L}}} \frac{\tau_{3}}{\tau_{0}}, \quad \nu=\frac{1}{8 \pi f_{\mathrm{L}} \tau_{3}}\left(\frac{32 \pi \tau_{0}}{5 \tau_{3}}\right)^{2 / 3} .
$$

There is a significant amount of literature on the computational requirements to search for compact binaries [321, 145, 277, 279]. The estimates for initial detectors are not alarming and it is possible to search for these systems online. Searches for these systems by the LSC (see, for example, [8]) employs a hexagonal lattice of templates [118] in the two-dimensional space of chirp times. For the best LIGO detectors we need several thousand templates to search for component masses in the range $\left[m_{\text {low }}, m_{\text {high }}\right]=[1,100] M_{\odot}[279]$. Decreasing the lower-end of the mass range leads to an increase in the number of templates that goes roughly as $m_{\text {low }}^{-8 / 3}$ and most current searches $[2,6]$ only begin at $m_{\text {low }}=1 M_{\odot}$, with the exception of one that looked for black hole binaries of primordial origin [7], in which the lower end of the search was $0.2 M_{\odot}$.

Inclusion of spins is only important when one or both of the components is rapidly spinning $[38,95]$. Spins effects are unimportant for neutron star binaries, for which the dimensionless spin parameter $q$, that is the ratio of its spin magnitude to the square of its mass, is tiny: $q=J_{\mathrm{NS}} / M_{\mathrm{NS}}^{2} \ll 1$. For ground-based detectors, even after including spins, the computational costs, while high, are not formidable and it should be possible to carry out the search on large computational clusters in real time [95]. Recently, the LSC has successfully carried out such a search [15].

5.1.3.2 Searching for Continuous Wave Signals. In the case of continuous waves $(\mathrm{CWs})$, the signal shape is pretty trivial: a sinusoidal oscillation with small corrections to take account of the slow spin-down of the neutron star/pulsar to account for the loss of angular momentum to gravitational waves and other radiation/particles. However, what leads to an enormous computational cost here is the Doppler modulation of the signal caused by Earth's rotation, the motion of the Earth around the solar system barycenter and the moon. The number of independent patches that we have to observe so as not to lose appreciable amounts of SNR can be worked out in the 
following manner. The baseline of a gravitational wave detector for CW sources is essentially $L=2 \times 1 A U \simeq 3 \times 10^{11} \mathrm{~m}$. For a source that emits gravitational waves at $100 \mathrm{~Hz}$, the wavelength of the radiation is $\lambda=3 \times 10^{6} \mathrm{~m}$, and the angular resolution $\Delta \theta$ of the antenna at an SNR of 1 is $\Delta \theta \simeq \lambda / L=10^{-5}$, or a solid angle of $\Delta \Omega \simeq(\Delta \theta)^{2}=10^{-10}$. In other words, the number of patches one should search for is $N_{\text {patches }} \sim 4 \pi / \Delta \Omega \simeq 10^{11}$. Moreover, for an observation that lasts for about a year $\left(T \simeq 3 \times 10^{7} \mathrm{~s}\right)$ the frequency resolution is $\Delta f=1 / T \simeq 3 \times 10^{-8}$. Searching over a frequency band of $300 \mathrm{~Hz}$, around the best sensitivity of the detector, gives the number of frequency bins to be about $10^{10}$. Thus, it is necessary to search over roughly $10^{11}$ patches in the sky for each of the $10^{10}$ frequency bins. This is a formidable task and one can only perform a matched filter search over a short period (days/weeks) of the data or over a restricted region in the sky, or just perform targeted searches for known objects such as pulsars, the galactic center, etc. [90].

The severe computational burden faced in the case of CW searches has led to the development of specialized searches that look for signals from known pulsars [5, 10, 12] using an efficient search algorithm that makes use of the known parameters $[115,150]$ and hierarchical algorithms that add power incoherently with the minimum possible loss in signal visibility [225, 4, 339, 11]. The most ambitious project in this regard is the Einstein@Home project [369]. The goal here is to carry out coherent searches for CW signals using wasted CPUs on idle computers at homes, offices and university departments around the world. The project has been successful in attracting a large number of subscriptions and provides the largest computational infrastructure to the LSC for the specific search of CW signals and the first scientific results from such are now being published by the LSC [13].

5.1.3.3 $\chi^{2}$ veto. Towards the end of Section 4.8 we discuss a powerful way of rejecting triggers, whose root cause is not gravitational wave signals but false alarms due to instrumental and environmental artifacts. In this section we will further quantify the $\chi^{2}$ veto [28] by using the scalar product introduced in the context of matched filtering.

The main problem with real data is that it can be glitchy in the form of high amplitude transients that might look like damped sinusoids. An inspiral signal and a template employed to detect it are both broadband signals. Therefore, the matched-filter SNR for such signals has contributions from a wide range of frequencies. However, the statistic of matched filtering, namely the SNR, is an integral over frequency and it is not sensitive to contributions from different frequency regions. Imagine dividing the frequency range of integration into a finite number of bins $f_{k} \leq f<f_{k+1}$, $k=1, \ldots, p$, such that their union spans the entire frequency band, $f_{1}=0$ and $f_{p+1}=\infty$, and further that the contribution to the SNR from each frequency bin is the same, namely,

$$
4 \int_{f_{k}}^{f_{k+1}} \frac{|\tilde{h}(f)|^{2}}{S_{h}(f)} \mathrm{d} f=\frac{4}{p} \int_{0}^{\infty} \frac{|\tilde{h}(f)|^{2}}{S_{h}(f)} \mathrm{d} f .
$$

Now, define the contribution to the matched filtering statistic coming from the $k$-th bin by [28]

$$
z_{k} \equiv\langle q, x\rangle_{k} \equiv 2 \int_{f_{k}}^{f_{k+1}}\left[\tilde{q}^{*}(f) \tilde{x}(f)+\tilde{q}(f) \tilde{x}^{*}(f)\right] \frac{\mathrm{d} f}{S_{h}(f)},
$$

where, as before, $\tilde{x}(f)$ and $\tilde{q}(f)$ are the Fourier transforms of the detector output and the template, respectively. Note that the sum $z=\sum_{k} z_{k}$ gives the full matched filtering statistic [28]:

$$
z=\langle q, x\rangle \equiv 2 \int_{0}^{\infty}\left[\tilde{q}^{*}(f) \tilde{x}(f)+\tilde{q}(f) \tilde{x}^{*}(f)\right] \frac{\mathrm{d} f}{S_{h}(f)} .
$$

Having chosen the bins and quantities $z_{k}$ as above, one can construct a statistic based on the 
measured SNR in each bin as compared to the expected value, namely

$$
\chi^{2}=p \sum_{k=1}^{p}\left(z_{k}-\frac{z}{p}\right)^{2} .
$$

When the background noise is stationary and Gaussian, the quantity $\chi^{2}$ obeys the well-known chi-square distribution with $p-1$ degrees of freedom. Therefore, the statistical properties of the $\chi^{2}$ statistic are known. Imagine two triggers with identical SNRs, but one caused by a true signal and the other caused by a glitch that has power only in a small frequency range. It is easy to see that the two triggers will have very different $\chi^{2}$ values; in the first case the statistic will be far smaller than in the second case. This statistic has served as a very powerful veto in the search for signals from coalescing compact binaries and it has been instrumental in cleaning up the data (see, e.g., $[2,6])$.

\subsection{Suboptimal filtering methods}

It is not always possible to compute the shape of the signal from a source. For instance, there is no computation, numerical or analytical, that reliably gives us the highly relativistic and nonlinear dynamics of gravitational collapse, the supernova that follows it and the emitted gravitational signal. The biggest problem here is the unknown physical state of the pre-supernova star and the complex physics that is involved in the collapse and explosion. Thus, matched filtering cannot be used to detect signals from supernovae.

Even when the waveform is known, the great variety in the shape of the emitted signals might render matched filtering ineffective. In binaries, in which one of the component masses is much smaller than the other, the smaller body will evolve on a highly precessing and in some cases eccentric orbit, due to strong spin-orbit coupling. Moreover, the radiation backreaction effects, which in the case of equal mass binaries are computed in an approximate way by averaging over an orbital time scale, should be computed much more accurately. The resulting motion of the small body in the Kerr spacetime of the larger body is extremely complicated, leading to a waveform that is rather complex and matched filtering would not be a practical approach.

Suboptimal methods can be used in such cases and they have a twofold advantage: they are less sensitive to the shape of the signal and are computationally significantly cheaper than matched filtering. Of course, the price is a loss in the SNR. The best suboptimal methods are sensitive to signal amplitudes a factor of two to three larger than that required by matched filtering and a factor of 10 to 30 in volume.

Most suboptimal techniques are one form of time-frequency transform or the other. They determine the presence or absence of a signal by comparing the power over a small volume in the time-frequency plane in a given segment of data to the average power in the same volume over a large segment of data. The time-frequency transform $q(\tau, f)$ of data $x(t)$ using a window $w(t)$ is defined as

$$
q(\tau, f)=\int_{-\infty}^{\infty} w(t-\tau) x(t) e^{2 \pi i f t} \mathrm{~d} t .
$$

The window function $w(t-\tau)$ is centered at $t=\tau$, and one obtains a time-frequency map by moving the window from one end of a data segment to the other. The window is not unique and the effectiveness of a window depends on the signal one is looking for. Once the time-frequency map is constructed, one can look for excess power (compared to average) in different regions [33], or look for certain patterns.

The method followed depends on the signal one is looking for. For instance, when looking for unknown signals, all that can be done is to look for a departure from averaged behavior in different regions of the map [33]. However, when some knowledge of the spectral and temporal content of 
the signal is known, it is possible to tune the algorithm to improve efficiency. The wavelet-based waveburst algorithm is one such example [217] that has been applied to search for unstructured bursts in LIGO data [9].

One can employ strategies that improve detection efficiency over a simple search for excess power. For example, chirping signals will leave a characteristic track in the time-frequency plane, with increasing frequency and power as a function of time. Time frequency map of a chirp signal buried in noisy data is shown in Figure 7. An algorithm that optimizes the search for specific shapes in the time-frequency plane is discussed in [187]. These and other methods have been applied to understand how to analyze LISA data [172, 389].
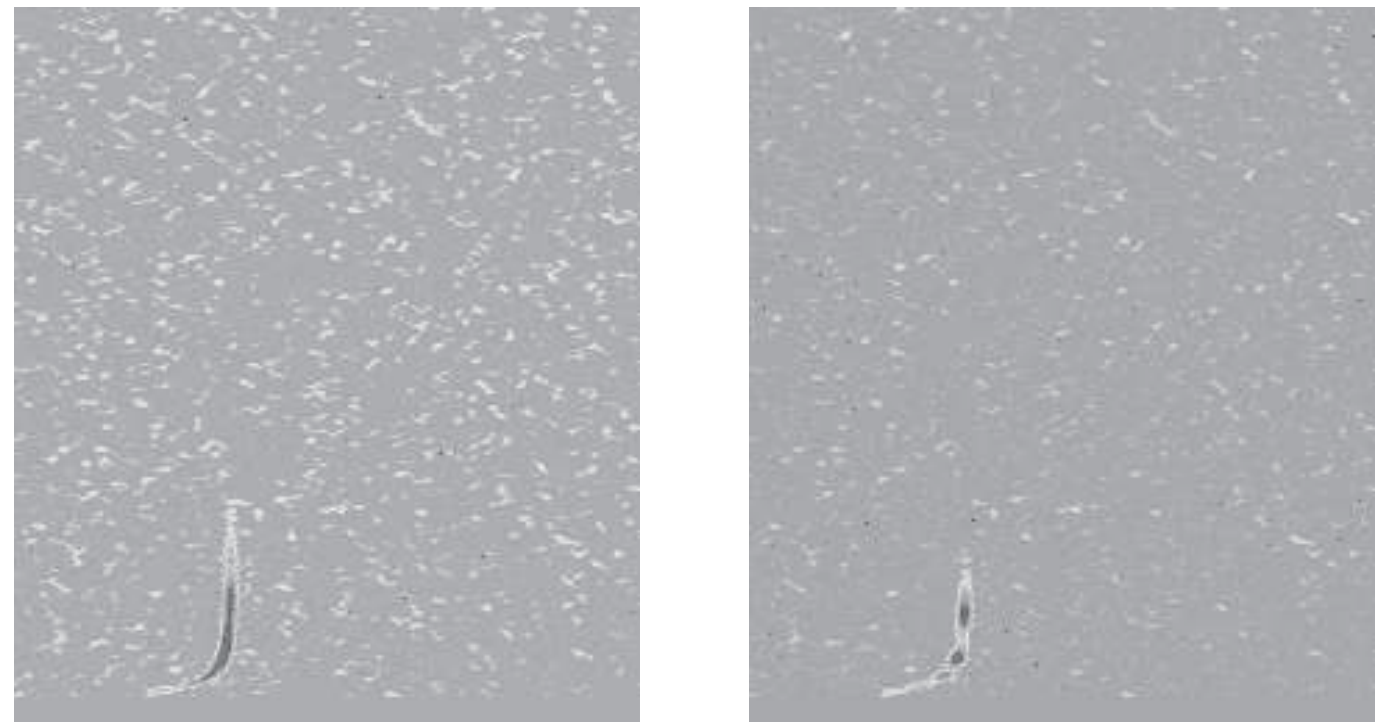

Figure 7: Time-frequency maps showing the track left by the inspiral of a small black hole falling into an SMBH as expected in LISA data. The left panel is for a central black hole without spin and the right panel is for a central black hole whose dimensionless spin parameter is $q=0.9$.

More recently, there has been a lot of progress in extending burst search algorithms for a network of detectors [113, 218], as well as exploring new Bayesian-based methods to search for unknown transients [335].

\subsection{Measurement of parameters and source reconstruction}

We have so far focused on the problem of detection and have not discussed parameter estimation in any concrete way. In principle, parameter estimation should not be considered to be distinct from detection. From a practical point of view, however, certain methods might be computationally efficient in detecting a signal but not necessarily the best for parameter estimation, while the best parameter estimation methods might not be computationally efficient. Thus, the problem of parameter estimation is often treated separately from detection.

The first thing to realize is that we can never be absolutely certain that a signal is present in a data train [159, 161]; we can only give confidence levels about its presence, which could be close to $100 \%$ for high values of the SNR. The next thing to realize is that, whatever the SNR may be, we cannot be absolutely certain about the true parameters of the signal: at best we can make an estimate and these estimates are given in a certain range. The width of the range depends on the confidence level required, being larger for higher confidence levels [159].

Living Reviews in Relativity

http: //www. livingreviews.org/lrr-2009-2 
Maximum likelihood estimates have long been used to measure the parameters of a known signal buried in noisy data. The method consists in maximizing the likelihood ratio - the ratio of the probability that a given signal is present in the data to the probability that the signal is absent [186, 159]. Maximum likelihood estimates are not always minimum uncertainty estimates, as has been particularly demonstrated in the case of binary inspiral signals by Balasubramanian, et al. [64, 65]. However, until recently, this is the method that has been very widely followed in the gravitational wave literature. But what is important to note is that maximum likelihood estimates are unbiased when the SNR is large ${ }^{3}$, and the mean of the distribution of measured values of the parameters will be centered around the true parameter values. This is an important quality that will be useful in our discussion below.

Bayesian estimates, which take into account any prior knowledge that may be available about the distribution of the source parameters, often give much better estimates and do not rely on the availability of an ensemble of detector outputs [340, 274]. However, they are computationally a lot more expensive than maximum likelihood estimates.

In any one measurement, any estimated parameters, however efficient, robust and accurate, are unlikely to be the actual parameters of the signal, since, at any finite SNR, noise alters the input signal. In the geometric language, the signal vector is being altered by the noise vector and our matched filtering aims at computing the projection of this altered vector onto the signal space. The true parameters are expected to lie within an ellipsoid of $p$ dimensions at a certain confidence level - the volume of the ellipsoid increasing with the confidence level at a given SNR but decreasing with the SNR at a given confidence level.

\subsubsection{Ambiguity function}

The ambiguity function, well known in the statistical theory of signal detection [186], is a very powerful tool in signal analysis: it helps one to assess the number of templates required to span the parameter space of the signal [321], to make estimates of variances and covariances involved in the measurement of various parameters, to compute biases introduced in using a family of templates whose shape is not the same as that of a family of signals intended to be detected, etc. We will see below how the ambiguity function can be used to compute the required number of templates. Towards the end of this section we will use the ambiguity function for the estimation of parameters.

The ambiguity function is defined (see Equation (91) below) as the scalar product of two normalized waveforms maximized over the initial phase of the waveform, in other words, the absolute value of the scalar product ${ }^{4}$. A waveform $e$ is said to be normalized if $\langle e, e\rangle^{1 / 2}=1$, where the inner product is inversely weighted by the PSD, as in Equation (79). Among other things, normalized waveforms help in defining signal strengths: a signal is said to be of strength $h_{0}$ if $h=h_{0} e$. Note that the optimal SNR for such a signal of strength $h_{0}$ is, $\langle h, h\rangle^{1 / 2}=h_{0}$.

Let $e(t ; \alpha)$, where $\alpha=\left\{\alpha^{i} \mid i=0, \ldots, p\right\}$ is the parameter vector comprised of $p+1$ parameters, denote a normalized waveform. It is conventional to choose the parameter $\alpha^{0}$ to be the lag $\tau$, which simply corresponds to a coordinate time when an event occurs and is therefore called an extrinsic parameter, while the rest of the $p$ parameters are called the intrinsic parameters and characterize the gravitational wave source.

Given two normalized waveforms $e(t ; \alpha)$ and $e(t ; \beta)$, whose parameter vectors are not necessarily

\footnotetext{
${ }^{3}$ How large the SNR should be to presume that there is no bias in the estimation of parameters depends on the number of parameter-space dimensions and strictly speaking the statement is true only in the limit as SNR $\rightarrow \infty$.

${ }^{4}$ Working with analytic signals $h(t)=a(t) e^{\phi(t)+i \phi_{0}}$, where $a(t)$ and $\phi(t)$ are the time-varying amplitude and phase of the signal, respectively, we see that the initial phase $\phi_{0}$ of the signal simply factors out as a constant phase in the Fourier domain and we can maximize over this initial phase by simply taking the absolute value of the scalar product of a template with a signal.
} 
the same, the ambiguity $\mathcal{A}$ is defined as

$$
\mathcal{A}(\alpha, \beta) \equiv|\langle e(\alpha), e(\beta)\rangle| \text {. }
$$

Since the waveforms are normalized, $\mathcal{A}(\alpha, \alpha)=1$ and $\mathcal{A}(\alpha, \beta)<1$, if $\alpha \neq \beta$. Here, $\alpha$ can be thought of as the parameters of a (normalized) template while $\beta$ those of a signal. With the template parameters $\alpha$ fixed, the ambiguity function is a function of $p$ signal parameters $\beta^{i}$, giving the SNR obtained by the template for different signals. The region in the signal parameter space for which a template obtains SNRs larger than a chosen value (called the minimal match [277]) is the span of that template. Template families should be chosen so that altogether they span the entire signal parameter space of interest with the least overlap of one other's spans. One can equally well interpret the ambiguity function as the SNR obtained for a given signal by filters of different parameter values.

It is clear that the ambiguity function is a local maximum at the "correct" set of parameters, $\beta=\alpha$. Search methods that vary $\beta$ to find the best fit to the parameter values make use of this property in one way or another. But the ambiguity function will usually have secondary maxima as a function of $\beta$ with fixed $\alpha$. If these secondaries are only slightly smaller than the primary maximum, then noise can lead to confusion: it can, at random, sometimes elevate a secondary and suppress a primary. These can lead to false measurements of the parameters. Search methods need to be designed carefully to avoid this as much as possible. One way would be to fit the known properties of the ambiguity function to an ensemble of maxima. This would effectively average over the noise on individual peaks and point more reliably to the correct one.

It is important to note that in the definition of the ambiguity function there is no need for the functional forms of the template and signal to be the same; the definition holds true for any signaltemplate pair of waveforms. Moreover, the number of template parameters need not be identical (and usually aren't) to the number of parameters characterizing the signal. For instance, a binary can be characterized by a large number of parameters, such as the masses, spins, eccentricity of the orbit, etc., while we may take as a model waveform the one involving only the masses. In the context of inspiral waves, $e(t ; \beta)$ is the exact general relativistic waveform emitted by a binary, whose form we do not know, while the template family is a post-Newtonian, or some other, approximation to it, that will be used to detect the true waveform. Another example would be signals emitted by spinning neutron stars, isolated or in binaries, whose time evolution is unknown, either because we cannot anticipate all the physical effects that affect their spin, or because the parameter space is so large that we cannot possibly take into account all of them in a realistic search.

Of course, in such cases we cannot compute the ambiguity function, since one of the arguments to the ambiguity function is unknown. These are, indeed, issues where substantial work is called for. What are all the physical effects to be considered so as not to miss out a waveform from our search? How to make a choice of templates when the functional form of templates is different from those of signals? For this review it suffices to assume that the signal and template waveforms are of identical shape and the number of parameters in the two cases is the same.

\subsubsection{Metric on the space of waveforms}

The computational cost of a search and the estimation of parameters of a signal afford a lucid geometrical picture developed by Balasubramanian et al. [65] and Owen [277]. Much of the discussion below is borrowed from their work.

Let $x_{k}, k=1,2, \ldots, N$, denote the discretely sampled output of a detector. The set of all possible detector outputs satisfy the usual axioms of a vector space. Therefore, $x_{k}$ can be thought of as an $N$-dimensional vector. It is more convenient to work in the continuum limit, in which case we have infinite dimensional vectors and the corresponding vector space. However, all the results

Living Reviews in Relativity

http: //www. livingreviews.org/lrr-2009-2 
are applicable to the realistic case in which detector outputs are treated as finite dimensional vectors.

Amongst all vectors, of particular interest are those corresponding to gravitational waves from a given astronomical source. While every signal can be thought of as a vector in the infinitedimensional vector space of the detector outputs, the set of all such signal vectors do not, by themselves, form a vector space. However, the set of all normed signal vectors (i.e., signal vectors of unit norm) form a manifold, the parameters of the signal serving as a coordinate system [64, 65, $277,279]$. Thus, each class of an astronomical source forms an $n$-dimensional manifold $\mathcal{S}_{n}$, where $n$ is the number of independent parameters characterizing the source. For instance, the set of all signals from a binary on a quasi-circular orbit inclined to the line of sight at an angle $\iota$, consisting of nonspinning black holes of masses $m_{1}$, and $m_{2}$, located a distance $D$ from the $\operatorname{Earth}^{5}$ initially in the direction $(\theta, \varphi)$ and expected to merge at a time $t_{C}$ with the phase of the signal at merger $\varphi_{C}$, forms a nine-dimensional manifold with coordinates $\left\{D, \theta, \varphi, m_{1}, m_{2}, t_{C}, \varphi_{C}, \iota, \psi\right\}$, where $\psi$ is the polarization angle of the signal. In the general case of a signal characterized by $n$ parameters we shall denote the parameters by $p^{\alpha}$, where $\alpha=1, \ldots, n$.

The manifold $\mathcal{S}_{n}$ can be endowed with a metric $g_{\alpha \beta}$ that is induced by the scalar product defined in Equation (79). The components of the metric in a coordinate system $p^{\alpha}$ are defined by ${ }^{6}$

$$
g_{\alpha \beta} \equiv\left\langle\partial_{\alpha} \hat{h}, \partial_{\beta} \hat{h}\right\rangle, \quad \partial_{\alpha} \hat{h} \equiv \frac{\partial \hat{h}}{\partial p^{\alpha}} .
$$

The metric can then be used on the signal manifold as a measure of the proper distance $\mathrm{d} \ell$ between nearby signals with coordinates $p^{\alpha}$ and $p^{\alpha}+\mathrm{d} p^{\alpha}$, that is signals $\hat{h}\left(p^{\alpha}\right)$ and $\hat{h}\left(p^{\alpha}+\mathrm{d} p^{\alpha}\right)$,

$$
\mathrm{d} \ell^{2}=g_{\alpha \beta} \mathrm{d} p^{\alpha} \mathrm{d} p^{\beta} .
$$

Now, by Taylor expanding $\hat{h}\left(p^{\alpha}+\mathrm{d} p^{\alpha}\right)$ around $p^{\alpha}$, and keeping only terms to second order in $\mathrm{d} p^{\alpha}$, it is straightforward to see that the overlap $\mathcal{O}$ of two infinitesimally close signals can be computed using the metric:

$$
\begin{aligned}
\mathcal{O}\left(\mathrm{d} p^{\alpha} ; p^{\alpha}\right) & \equiv\left\langle\hat{h}\left(p^{\alpha}\right), \hat{h}\left(p^{\alpha}+\mathrm{d} p^{\alpha}\right)\right\rangle \\
& =1-\frac{1}{2} g_{\alpha \beta} \mathrm{d} p^{\alpha} \mathrm{d} p^{\beta} .
\end{aligned}
$$

The metric on the signal manifold is nothing but the well-known Fisher information matrix usually denoted $\Gamma_{\alpha \beta}$, (see, e.g., $[186,283]$ ) but scaled down by the square of the SNR, i.e., $g_{\alpha \beta}=$ $\rho^{-2} \Gamma_{\alpha \beta}$. The information matrix is itself the inverse of the covariance matrix $C_{\alpha \beta}$ and is a very useful quantity in signal analysis.

\subsubsection{Covariance matrix}

Having defined the metric, we next consider the application of the geometric formalism in the estimation of statistical errors involved in the measurement of the parameters. We closely follow the notation of Finn and Chernoff [159, 161, 114].

Let us suppose a signal of known shape with parameters $p^{\alpha}$ is buried in background noise that is Gaussian and stationary. Since the signal shape is known, one can use matched filtering to dig the signal out of the noise. The measured parameters $\bar{p}^{\alpha}$ will, in general, differ from the true

\footnotetext{
${ }^{5}$ Even though we deal with normed signals (which amounts to fixing $D$ ), astrophysical gravitational wave signals are characterized by this additional parameter.

${ }^{6}$ We have followed the definition of the metric as is conventional in parameter estimation theory (see, e.g., [159, 161, 114, 65]), which differs from that used in template placement algorithms (see, e.g., [277]) by a factor of two. This difference will impact the relationship between the metric and the match, as will be apparent in what follows.
} 
parameters of the signal ${ }^{7}$. Geometrically speaking, the noise vector displaces the signal vector and the process of matched filtering projects the (noise + signal) vector back on to the signal manifold. Thus, any nonzero noise will make it impossible to measure the true parameters of the signal. The best one can hope for is a proper statistical estimation of the influence of noise.

The posterior probability density function $\mathcal{P}$ of the parameters $\bar{p}^{\alpha}$ is given by a multivariate Gaussian distribution ${ }^{8}$ :

$$
\mathcal{P}\left(\Delta p^{\alpha}\right) \mathrm{d}^{n} \Delta p=\frac{\mathrm{d}^{n} \Delta p}{(2 \pi)^{n / 2} \sqrt{C}} \exp \left[-\frac{1}{2} C_{\alpha \beta}^{-1} \Delta p^{\alpha} \Delta p^{\beta}\right],
$$

where $n$ is the number of parameters, $\Delta p^{\alpha}=p^{\alpha}-\bar{p}^{\alpha}$, and $C_{\alpha \beta}$ is the covariance matrix, $C$ being its determinant. Noting that $C_{\alpha \beta}^{-1}=\rho^{2} g_{\alpha \beta}$, we can rewrite the above distribution as

$$
\mathcal{P}\left(\Delta p^{\alpha}\right) \mathrm{d}^{n} \Delta p=\frac{\rho^{n} \sqrt{g} \mathrm{~d}^{n} \Delta p}{(2 \pi)^{n / 2}} \exp \left[-\frac{\rho^{2}}{2} g_{\alpha \beta} \Delta p^{\alpha} \Delta p^{\beta}\right],
$$

where we have used the fact that $C=1 /\left(\rho^{2 n} g\right), g$ being the determinant of the metric $g_{\alpha \beta}$. Note that if we define new parameters $p^{\alpha}=\rho p^{\alpha}$, then we have exactly the same distribution function for all SNRs, except that the deviations $\Delta p^{\alpha}$ are scaled by $\rho$.

Let us first specialize to one dimension to illustrate the region of the parameter space with which one should associate an event at a given confidence level. In one dimension the distribution of the deviation from the mean of the measured value of the parameter $p$ is given by

$$
\mathcal{P}(\Delta p) \mathrm{d} \Delta p=\frac{\mathrm{d} \Delta p}{\sqrt{2 \pi} \sigma} \exp \left(-\frac{\Delta p^{2}}{2 \sigma^{2}}\right)=\frac{\rho \sqrt{g_{p p}} \mathrm{~d} \Delta p}{\sqrt{2 \pi}} \exp \left(-\frac{\rho^{2}}{2} g_{p p} \Delta p^{2}\right),
$$

where, analogous to the $n$-dimensional case, we have used $\sigma^{2}=1 /\left(\rho^{2} g_{p p}\right)$. Now, at a given SNR, what is the volume $V_{P}$ in the parameter space, such that the probability of finding the measured parameters $\bar{p}$ inside this volume is $P$ ? This volume is defined by

$$
P=\int_{\Delta p \in V_{P}} \mathcal{P}(\Delta p) \mathrm{d} \Delta p
$$

Although $V_{P}$ is not unique, it is customary to choose it to be centered around $\Delta p=0$ :

$$
P=\int_{(\Delta p / \sigma)^{2} \leq r^{2}(P)} \frac{\mathrm{d} \Delta p}{\sqrt{2 \pi} \sigma} \exp \left(-\frac{\Delta p^{2}}{2 \sigma^{2}}\right)=\int_{\rho^{2} g_{p p} \Delta p^{2} \leq r^{2}(P)} \frac{\rho \sqrt{g_{p p}} \mathrm{~d} \Delta p}{\sqrt{2 \pi}} \exp \left(-\frac{\rho^{2} g_{p p} \Delta p^{2}}{2}\right),
$$

where, given $P$, the above equation can be used to solve for $r(P)$ and it determines the range of integration: $-r \sigma \leq \Delta p \leq r \sigma$. For instance, the volumes $V_{P}$ corresponding to $P \simeq 0.683,0.954,0.997, \ldots$, are the familiar intervals $[-\sigma, \sigma],[-2 \sigma, 2 \sigma],[-3 \sigma, 3 \sigma], \ldots$, and the corresponding values of $r$ are 1,2 , 3. Since $\sigma=1 / \sqrt{\rho^{2} g_{p p}}$, we see that in terms of $g_{p p}$ the above intervals translate to

$$
\frac{1}{\rho}\left[-\frac{1}{\sqrt{g_{p p}}}, \frac{1}{\sqrt{g_{p p}}}\right], \frac{1}{\rho}\left[-\frac{2}{\sqrt{g_{p p}}}, \frac{2}{\sqrt{g_{p p}}}\right], \frac{1}{\rho}\left[-\frac{3}{\sqrt{g_{p p}}}, \frac{3}{\sqrt{g_{p p}}}\right], \ldots
$$

Thus, for a given probability $P$, the volume $V_{P}$ shrinks as $1 / \rho$. The maximum distance $d_{\max }$ within which we can expect to find "triggers" at a given $P$ depends inversely on the SNR $\rho$ : $d \ell=\sqrt{g_{p p} \Delta p^{2}}=r / \rho$. Therefore, for $P \simeq 0.954, r=2$ and at an SNR of 5 the maximum distance

\footnotetext{
${ }^{7}$ In what follows we shall use an over-line to distinguish the measured parameters from the true parameters $p^{\alpha}$.

${ }^{8} \mathrm{~A}$ Bayesian interpretation of $\mathcal{P}\left(\Delta p^{\alpha}\right)$ is the probability of having the true signal parameters lie somewhere inside the ellipsoidal volume centered at the Maximum Likelihood point $\bar{p}^{\alpha}$.
} 
is 0.4 , which corresponds to a match of $\epsilon=1-\frac{1}{2} d \ell^{2}=0.92$. In other words, in one dimension $95 \%$ of the time we expect our triggers to come from templates that have an overlap greater than or equal to 0.92 with the buried signal when the SNR is five. This interpretation in terms of the match is a good approximation as long as $d \ell \ll 1$, which will be true for large SNR events. However, for weaker signals and/or greater values of $P$ we can't interpret the results in terms of the match, although Equation (98) can be used to determine $r(P)$. As an example, at $P \simeq 0.997, r=3$ and at an SNR of $\rho=4$, the maximum distance is $d \ell=0.75$ and the match is $\epsilon=23 / 32 \simeq 0.72$, which is significantly smaller than one and the quadratic approximation is not good enough to compute the match.

These results generalize to $n$ dimensions. In $n$-dimensions the volume $V_{P}$ is defined by

$$
P=\int_{\Delta p^{\alpha} \in V_{P}} \mathcal{P}\left(\Delta p^{\alpha}\right) \mathrm{d}^{n} \Delta p .
$$

Again, $V_{P}$ is not unique but it is customary to center the volume around the point $\Delta p^{\alpha}=0$ :

$$
P=\int_{\rho^{2} g_{\alpha \beta} \Delta p^{\alpha} \Delta p^{\beta} \leq r^{2}(P, n)} \frac{\rho^{n} \sqrt{g} \mathrm{~d}^{n} \Delta p}{(2 \pi)^{n / 2}} \exp \left[-\frac{\rho^{2}}{2} g_{\alpha \beta} \Delta p^{\alpha} \Delta p^{\beta}\right] .
$$

Given $P$ and the parameter space dimension $n$, one can iteratively solve the above equation for $r(P, n)$. The volume $V_{P}$ is the surface defined by the equation

$$
g_{\alpha \beta} \Delta p^{\alpha} \Delta p^{\beta}=\left(\frac{r}{\rho}\right)^{2} .
$$

This is the equation of an $n$-dimensional ellipsoid whose size is defined by $r / \rho$. For a given $r$ (which determines the confidence level), the size of the ellipsoid is inversely proportional to the SNR, the volume decreasing as $\rho^{n}$. However, the size is not small enough for all combinations of $P$ and $\rho$ to interpret the distance from the center of the ellipsoid to its surface, in terms of the overlap or match of the signals at the two locations, except when the distance is close to zero. This is because the expression for the match in terms of the metric is based on the quadratic approximation, which breaks down when the matches are small. However, the region defined by Equation (103) always corresponds to the probability $P$ and there is no approximation here (except that the detector noise is Gaussian).

When the SNR $\rho$ is large and $1-P$ is not close to zero, the triggers are found from the signal with matches greater than or equal to $1-\frac{r^{2}(P, n)}{2 \rho^{2}}$. Table 2 lists the value of $r$ for several values of $P$ in one, two and three-dimensions and the minimum match $\epsilon_{\mathrm{MM}}$ for SNRs 5, 10 and 20.

Table 2 should be interpreted in light of the fact that triggers come from an analysis pipeline in which the templates are laid out with a certain minimal match and one cannot, therefore, expect the triggers from different detectors to be matched better than the minimal match.

From Table 2, we see that, when the SNR is large (say greater than about 10), the dependence of the match $\epsilon_{\mathrm{MM}}$ on $n$ is very weak; in other words, irrespective of the number of dimensions, we expect the match between the trigger and the true signal (and for our purposes the match between triggers from different instruments) to be pretty close to 1 , and mostly larger than a minimal match of about 0.95 that is typically used in a search. Even when the SNR is in the region of 5, for low $P$ again there is a weak dependence of $\epsilon_{\mathrm{MM}}$ on the number of parameters. For large $P$ and low SNR, however, the dependence of $\epsilon_{\mathrm{MM}}$ on the number of dimensions becomes important. At an SNR of 5 and $P \simeq 0.997, \epsilon_{\mathrm{MM}}=0.91,0.87,0.85$ for $n=1,2,3$ dimensions, respectively.

Bounds on the estimation computed using the covariance matrix are called Cramér-Rao bounds. Cramér-Rao bounds are based on local analysis and do not take into consideration the effect of distant points in the parameter space on the errors computed at a given point, such as the 
Table 2: The value of the (squared) distance $d \ell^{2}=r^{2} / \rho^{2}$ for several values of $P$ and the corresponding smallest match that can be expected between templates and the signal at different values of the SNR.

\begin{tabular}{c|cc|cc|cc}
\hline & $P=0.683$ & \multicolumn{2}{c}{$P=0.954$} & \multicolumn{2}{c}{$P=0.997$} \\
\hline$\rho$ & $d \ell^{2}$ & $\epsilon_{\mathrm{MM}}$ & $d \ell^{2}$ & $\epsilon_{\mathrm{MM}}$ & $d \ell^{2}$ & $\epsilon_{\mathrm{MM}}$ \\
\hline \multicolumn{7}{c}{$n=1$} \\
\hline 5 & 0.04 & 0.9899 & 0.16 & 0.9592 & 0.36 & 0.9055 \\
10 & 0.01 & 0.9975 & 0.04 & 0.9899 & 0.09 & 0.9772 \\
20 & 0.0025 & 0.9994 & 0.01 & 0.9975 & 0.0225 & 0.9944 \\
\hline \multicolumn{7}{c}{$n=2$} \\
\hline 5 & 0.092 & 0.9767 & 0.2470 & 0.9362 & 0.4800 & 0.8718 \\
10 & 0.023 & 0.9942 & 0.0618 & 0.9844 & 0.1200 & 0.9695 \\
20 & 0.00575 & 0.9986 & 0.0154 & 0.9961 & 0.0300 & 0.9925 \\
\hline \multicolumn{7}{c}{$n=3$} \\
\hline 5 & 0.1412 & 0.9641 & 0.32 & 0.9165 & 0.568 & 0.8462 \\
10 & 0.0353 & 0.9911 & 0.08 & 0.9798 & 0.142 & 0.9638 \\
20 & 0.00883 & 0.9978 & 0.02 & 0.9950 & 0.0355 & 0.9911 \\
\hline
\end{tabular}

secondary maxima in the likelihood. Though the Cramér-Rao bounds are in disagreement with maximum likelihood estimates, global analysis, taking the effect of distant points on the estimation of parameters, does indeed give results in agreement with maximum likelihood estimation as shown by Balasubramanian and Dhurandhar [63].

\subsubsection{Bayesian inference}

A good example of an efficient detection algorithm that is not a reliable estimator is the timefrequency transform of a chirp. For signals that are loud enough, a time-frequency transform of the data would be a very effective way of detecting the signal, but the transform contains hardly any information about the masses, spins and other information about the source. This is because the time-frequency transform of a chirp is a mapping from the multi-dimensional (17 in the most general case) space of chirps to just the two-dimensional space of time and frequency. Even matched filtering, which would use templates that are defined on the full parameter space of the signal, would not give the parameters at the expected accuracy. This is because the templates are defined only at a certain minimal match and might not resolve the signal well enough, especially for signals that have a high SNR.

In recent times Bayesian inference techniques have been applied with success in many areas in astronomy and cosmology. These techniques are probably the most sensible way of estimating the parameters, and the associated errors, but cannot be used to efficiently search for signals. Bayesian inference is among the simplest of statistical measures to state, but is not easy to compute and is often subject to controversies. Here we shall only discuss the basic tenets of the method and refer the reader for details to an excellent treatise on the subject (see, e.g., Sivia [340]).

To understand the chief ideas behind Bayesian inference, let us begin with some basic concepts in probability theory. Given two hypothesis or statements $A$ and $B$ about an observation, let $P(A, B)$ denote the joint probability of $A$ and $B$ being true. For the sake of clarity, let $A$ denote a statement about the universe and $B$ some observation that has been made. Now, the joint probability can be expressed in terms of the individual probability densities $P(A)$ and $P(B)$ and

Living Reviews in Relativity

http://www. livingreviews.org//rr-2009-2 
conditional probability densities $P(A \mid B)$ and $P(B \mid A)$ as follows:

$$
P(A, B)=P(A) P(B \mid A) \quad \text { or } \quad P(A, B)=P(B) P(A \mid B) .
$$

The first of these equations says the joint probability of $A$ and $B$ both being true is the probability that $A$ is true times the probability that $B$ is true given that $A$ is true and similarly the second. We can use the above equations to arrive at Bayes theorem:

$$
P(B) P(A \mid B)=P(A) P(B \mid A) \quad \text { or } \quad P(A \mid B)=\frac{P(A) P(B \mid A)}{P(B)} .
$$

The left-hand side of the above equation can be interpreted as a statement about $A$ (the universe) given $B$ (the data). This is the posterior probability density. The right-hand side contains $P(B \mid A)$, which is the probability that $B$ is obtained given that $A$ is true and is called the likelihood, $P(A)$, which is the probability of $A$, called the prior probability of $A$, and $P(B)$ (the prior of $B$ ), which is simply a normalizing constant often ignored in Bayesian analysis.

For instance, if $A$ denotes the statement it is going to rain and $B$ the amount of humidity in the air then the above equation gives us the posterior probability that it rains when the air contains a certain amount of humidity. Clearly, the posterior depends on what is the likelihood of the air having a certain humidity when it rains and the prior probability of rain on a given day. If the prior is very small (as it would be in a desert, for example) then you would need a rather large likelihood for the posterior to be large. Even when the prior is not so small, say a $50 \%$ chance of rain on any given day (as it would be if you are in Wales), the likelihood has to be large for posterior probability to say something about the relationship between the level of humidity and the chance of rain.

As another example, and more relevant to the subject of this review, let $s$ be the statement the data contains a chirp (signal), $n$ the statement the data contains an instrumental transient, (noise), and let $t$ be a test that is performed to infer which of the two statements above are true. Let us suppose $t$ is a very good test, in that it discriminates between $s$ and $n$ very well, and say the detection probability is as high as $P(t \mid s)=0.95$ with a low false alarm rate $P(t \mid n)=0.05$ (note that $P(t \mid s)$ and $P(t \mid n)$ need not necessarily add up to 1 ). Also, the expected event rate of a chirp during our observation is low, $P(s)=10^{-5}$, but the chance of an instrumental transient is relatively large, $P(n)=0.01$. We are interested in knowing what the posterior probability of the data containing a chirp is, given that the test has been passed. By Bayes theorem this is

$$
P(s \mid t)=\frac{P(t \mid s) P(s)}{P(t)}=\frac{P(t \mid s) P(s)}{P(t \mid s) P(s)+P(t \mid n) P(n)},
$$

where $P(t)$ (the probability of the test being positive) is taken to result from either the chirp or the instrumental transient. Substituting for various quantities in the above equation we find

$$
P(s \mid t)=\frac{0.95 \times 10^{-5}}{0.95 \times 10^{-5}+0.05 \times 0.01} \simeq 0.02 .
$$

There is only a $2 \%$ chance that the data really contains a chirp when the test was taken. On the contrary, for the same data we find that the chance of an instrumental transient for a positive test result is $P(n \mid t) \sim 98 \%$. Thus, though there is a high (low) probability for the test to be positive in the presence of a signal (noise) when the test is indeed positive, we cannot necessarily conclude that a signal is present. This is not surprising since the prior probability of the signal being present is very low. The situation can be remedied by designing a test that gives a far lower probability for the test to give a positive result in the case of an instrumental transient (i.e., a very low false alarm rate). 
Thus, Bayesian inference neatly folds the prior knowledge about sources in the estimation process. One might worry that the outcome of a measurement process would be seriously biased by our preconception of the prior. To understand this better, let us rewrite Equation (106) as follows:

$$
P(s \mid t)=\frac{1}{1+P(t \mid n) P(n) / P(t \mid s) P(s)}=\frac{1}{1+L_{\mathrm{NS}} p_{\mathrm{SN}}},
$$

where $L_{\mathrm{NS}}=P(t \mid n) / P(t \mid s)$ is the ratio of the two likelihoods and $p_{\mathrm{SN}}=P(s) / P(n)$ is the ratio of the priors. The latter is not in the hands of a data analyst; it is determined by the nature of the source being searched for and the property of the instrument. The only way an analyst can make the posterior probability large is by choosing a test that gives a small value for the ratio of the two likelihoods. When $L_{\mathrm{NS}} \ll p_{\mathrm{SN}}$ (i.e., the likelihood of the test being positive when the signal is present is far larger, depending on the priors, than when the transient is present) the posterior will be close to unity.

The above example tells us why we have to work with unusually-small false-alarm probability in the case of gravitational wave searches. For instance, to search for binary coalescences in groundbased detectors we use a (power) SNR threshold of about 30 to 50. This is because the expected event rate is about 0.04 per year.

Computing the posterior involves multi-dimensional integrals and these are highly expensive computationally, when the number of parameters involved is large. This is why it is often not possible to apply Bayesian techniques to continuously streaming data; it would be sensible to reserve the application of Bayesian inference only for candidate events that are selected from inexpensive analysis techniques. Thus, although Bayesian analysis is not used in current detection pipelines, there has been a lot of effort in evaluating its ability to search for $[115,348,122,120]$ and measure the parameters of [116, 121, 377] a signal and in follow-up analysis [378]. 


\section{Physics with Gravitational Waves}

Classical general relativity has passed all possible experimental and observational tests so far. The theory is elegant, self-consistent and mathematically complete (i.e., its equations are, in principle, solvable). However, theorists are uncomfortable with general relativity because it has so far eluded all efforts of quantization, making it a unique modern theory, whose quantum mechanical analogue is unknown. Although general relativity arises as a by-product in certain string theories, the physical relevance of such theories is unclear. Therefore, it has been proposed that general relativity is a low-energy limit of a more general theory, which in itself is amenable to both quantization and unification. There are also other theoretical motivations to look for modifications of general relativity or new theories of gravity. While there are some alternative candidates (including the Brans-Dicke theory), none has predictions that contradict general relativistic predictions in linear and mildly nonlinear gravitational fields. More precisely, the extra parameters of these other theories of gravity are constrained by the present experimental and astronomical observations, however, they are expected to significantly deviate from general relativistic predictions under conditions of strong gravitational fields.

Gravitational wave observations provide a unique opportunity to test strongly nonlinear and highly relativistic gravity and hence provide an unprecedented testbed for confronting different theories of gravity. Every nonlinear gravitational effect in general relativity will have a counterpart in alternative theories and therefore a measurement of such an effect would provide an opportunity to compare the performance of general relativity with its competitors. Indeed, a single measurement of the full polarization of an incident gravitational wave can potentially rule out general relativity. This is a field that would benefit from an in-depth study. What we are lacking is a systematic study of higher-order post-Newtonian effects in alternative theories of gravity. For instance, we do not know how tails of gravitational waves or tails of tails would appear in any theory other than general relativity.

In what follows we present strong field tests of general relativity afforded by future gravitational wave observations. We will begin with observations of single black holes followed by black hole binaries (more generally, coalescing binaries of compact objects).

\subsection{Speed of gravitational waves}

Association of a gravitational wave event with an electromagnetic event, such as the observation of a gamma or X-ray burst coincidentally with a gravitational wave event, would help to deduce the speed of gravitational waves to a phenomenal accuracy. The best candidate sources for the simultaneous observations of both are the well-known extra-galactic gamma-ray bursts (GRBs). Depending on the model that produces the GRB, the delay between the emission of a GRB and gravitational waves might be either a fraction of a second (as in GRBs generated by internal shocks in a fireball [306]) or 100's of seconds (as in GRBs generated when the fireball is incident on an external medium [256]). It is unlikely that high-redshift gamma-ray observations will be visible in the gravitational wave band, since the amplitude of gravitational waves might be rather low. However, advanced detectors might see occasional low-redshift events, especially if the GRB is caused by black-hole-neutron-star mergers. Third generation detectors would be sensitive to such events up to $z=2$. A single unambiguous association can verify the speed of gravitational waves relative to light to a fantastic precision.

For instance, even a day's delay in the arrival times of gravitational and electromagnetic radiation from a source at a distance of one giga light year (distance to a low-redshift GRB detectable by advanced detectors) would determine the relative speeds to better than one part in $10^{11}$ ( 1 day $/ 10^{9}$ yr $\sim 3 \times 10^{-12}$ ). Coincident detection of GRBs and gravitational waves would require good timing accuracy to determine the direction of the source so that astronomical observations 
of associated gamma rays (and afterglows in other spectral bands) can be made. Consequently, gravitational wave antennas around the globe will have to make a coincident detection of the event.

If the speed of gravitational waves is less than that of light, then this could indicate that the graviton has an effective nonzero mass.

This would have other observable effects, in particular dispersion; different frequencies should move at different speeds. Will [393] pointed out that LISA's observations of coalescences of SMBHs at high redshifts will place extremely tight constraints on dispersion, and may, therefore, indirectly set the best available limits on the speed of gravitational waves. This and other bounds on the graviton mass are discussed in Section 6.6.1.

\subsection{Polarization of gravitational waves}

As noted in Section 2, in Einstein's theory gravitational waves have two independent polarizations, usually denoted $h_{+}$and $h_{\times}$[259]. A general wave will be a linear combination of both. Rotating sources typically emit both polarizations with a phase delay between them, leading to elliptical polarization patterns. Depending on the nature of the source such polarizations can be detected either with a single detector (in the case of continuous wave sources) or with a network of detectors (in the case of burst sources).

While Einstein's general relativity predicts only two independent polarizations, there are other theories of gravitation in which there are additional states of polarization. For instance, in FierzeJordan-Brans-Dicke theory [394] there are four polarization degrees of freedom more than in Einstein's theory. Therefore, an unambiguous determination of the polarization of the waves will be of fundamental importance.

In the case of a burst source, to determine two polarization states, source direction and amplitude requires three detectors, observing other polarizations would require the use of more than three detectors (see, for example, Will [394]). The scalar polarization mode of Brans-Dicke, for example, expands a transverse ring of test particles without changing its shape. This is the breathing mode, or monopole polarization. If such a wave is incident from above on an interferometer, it will not register at all. But if it comes in along one of the arms, then, since it acts transversely, it will affect only the other arm and leave a signal. If the wave is seen with enough detectors, then it is possible to determine that it has scalar polarization. Note that a measurement such as this can make a qualitative change in physics: a single measurement could put general relativity in jeopardy.

Polarization measurements have an important application in astronomy. The polarization of the waves contains orientation information. For example, a binary system emits purely circular polarization along the angular momentum axis, but purely linear polarization in its equatorial plane. By measuring the polarization of waves from a binary (or from a spinning neutron star) one can determine the orientation and inclination of its spin axis. This is a piece of information that is usually very hard to extract from optical observations. We will return to this discussion in Section 7.1.1.

\subsection{Gravitational radiation reaction}

In 1974, Hulse and Taylor discovered the first double neutron star binary PSR B1913+16, a system in which the emission of gravitational radiation has an observable effect [200,356]. General relativity predicts that the loss of energy and angular momentum due to the emission of gravitational waves should cause the period of the system to decrease and, by carefully monitoring the orbital period of the binary, that it would be possible to measure the rate at which the period changes. The rate at which the period decays can be computed using the quadrupole formula for

Living Reviews in Relativity

http: //www . livingreviews . org/lrr-2009-2 
the luminosity of the emitted radiation combined with the energy-balance equation; namely that the energy carried away by the waves comes at the expense of the binding energy of the system.

For a binary consisting of stars of masses $m_{1}$ and $m_{2}$, in an orbit of eccentricity $e$ and period $P_{b}$, the period decay is given by the generalization of Equation (32) [290]:

$$
\dot{P}_{b}=-\frac{192 \pi}{5}\left(\frac{2 \pi \mathcal{M}}{P_{b}}\right)^{5 / 3}\left(1+\frac{73}{24} e^{2}+\frac{37}{96} e^{4}\right)\left(1-e^{2}\right)^{-7 / 2},
$$

where we recall that

$$
\mathcal{M}=\left(m_{1} m_{2}\right)^{3 / 5}\left(m_{1}+m_{2}\right)^{-1 / 5}=\mu^{3 / 5} M^{2 / 5}
$$

is the chirpmass of the binary that we defined in Equation (31). (In the third expression here, $\mu$ is the reduced mass of the binary and $M$ its total mass.) Since the masses of the binary and the eccentricity of the orbit can be measured by other means, one can use these parameters in the above equation to infer the rate at which the period is predicted to decrease according to general relativity. For the Hulse-Taylor binary the relevant values are: $m_{1}=1.4414 M_{\odot}, m_{2}=1.3867 M_{\odot}$, $e=0.6171338, P_{b}=2.790698 \times 10^{4} \mathrm{~s}$. The predicted value $\dot{P}_{b}{ }^{\mathrm{GR}}=-(2.40242 \pm 0.00002) \times 10^{-12}$, while the observed period decay (after subtracting the apparent decay due to the acceleration of the pulsar in the gravitational field of our galaxy, as described in Section 3.4.3) is $\dot{P}_{b}^{\text {Obs }}=$ $-(2.4056 \pm 0.0051) \times 10^{-12}$ and the two are in agreement to better than a tenth of a percent [394].

Observation of the decay of the orbital period in PSR B1913+16 is an unambiguous direct observation of the effect of gravitational radiation backreaction on the dynamics of the system. PSR B1913+16 was the first system in which the effect of gravitational radiation reaction force was measured. In 2004, a new binary pulsar PSR J0737-3039 was discovered [101, 247]. J0737 is in a tighter orbit than PSR B1913+16; with an orbital period of only $2.4 \mathrm{hrs}$, the orbit is shrinking by about $7 \mathrm{~mm}$ each day in good agreement with the general relativistic prediction. Several other systems are also known [242]. In Sections 6.5, 6.5.2 and 6.5.3 we will discuss in some detail the dynamics of relativistic binaries and the radiation reaction as predicted by post-Newtonian theory and numerical relativity simulations.

\subsection{Black hole spectroscopy}

An important question relating to the structure of a black hole is its stability. Studies that began in the 1970s [307, 381, 382, 398, 296, 357, 358, 297] showed that a black hole is stable under external perturbation. A formalism was developed to study how a black hole responds to generic external perturbations, which has come to be known as black hole perturbation theory [112]. What we now know is that a distorted Kerr black hole relaxes to its axisymmetric state by partially emitting the energy in the distortion as gravitational radiation. The radiation consists of a superposition of QNMs, whose frequency and damping time depend uniquely on the mass $M$ and spin angular momentum $J$ of the parent black hole and not on the nature of the external perturbation. The amplitudes and damping times of different modes, however, are determined by the details of the perturbation and are not easy to calculate, except in some simple cases.

The uniqueness of the QNMs is related to the "no-hair" theorem of general relativity according to which a black hole is completely specified by its mass and $\operatorname{spin}^{9}$. Thus, observing QNMs would not only confirm the source to be a black hole, but would be an unambiguous proof of the uniqueness theorem of general relativity.

The end state of a black hole binary will lead to the formation of a single black hole, which is initially highly distorted. Therefore, one can expect coalescing black holes to end their lives

\footnotetext{
${ }^{9} \mathrm{~A}$ black hole can, in principle, carry an electric charge in addition to mass and spin angular momentum. However, astrophysical black holes are believed to be electrically neutral
} 
with the emission of QNM radiation, often called ringdown radiation. It was realized quite early on [164] that the energy emitted during the ringdown phase of a black-hole-binary coalescence could be pretty large. Although, the initial quantitative estimates [164] have proven to be rather high, the qualitative nature of the prediction has proven to be correct. Indeed, numerical relativity simulations show that about 1-2\% of a binary's total mass would be emitted in QNMs [298]. The effective one-body (EOB) model $[96,97]$, the only analytical treatment of the merger dynamics, gives the energy in the ringdown radiation to be about $0.7 \%$ of the total mass, consistent with numerical results. Thus, it is safe to expect that the ringdown will be as luminous an event as the inspiral and the merger phases. The fact that QNMs can be used to test the no-hair theorem puts a great emphasis on understanding their properties, especially the frequencies, damping times and relative amplitudes of the different modes that will be excited during the merger of a black hole binary and how accurately they can be measured.
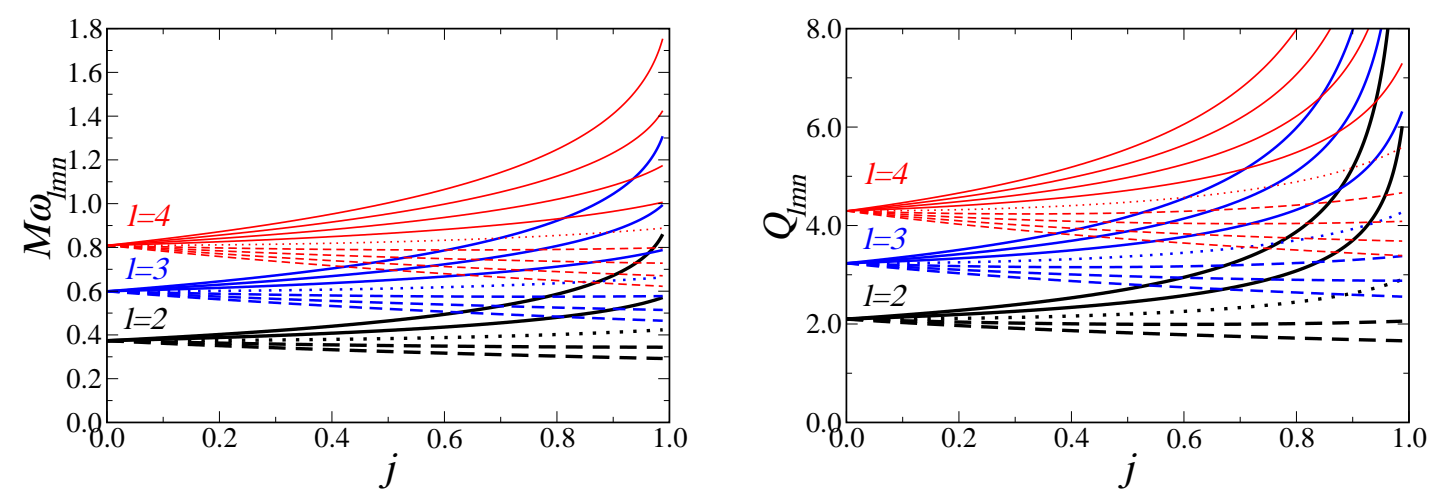

Figure 8: Normal mode frequencies (left) and corresponding quality factors (right) of fundamental modes with $l=2,3,4$, as a function of the dimensionless black hole spin $j$, for different values of $m=l, \ldots, 0, \ldots-l$ (for each $l$, different line styles from top to bottom correspond to decreasing values of $m$ ). Figure reprinted with permission from [76]. (c) The American Physical Society.

QNMs are characterized by a complex frequency $\omega$ that is determined by three "quantum" numbers, $(l, m, n)$ (see, e.g., [76]). Here $(l, m)$ are indices that are similar to those for standard spherical harmonics. For each pair of $(l, m)$ there are an infinitely large number of resonant modes characterized by another integer $n$. The time dependence of the oscillations is given by $\exp (i \omega t)$, where $\omega$ is a complex frequency, its real part determining the mode frequency and the imaginary part (which is always positive) giving the damping time: $\omega=\omega_{l m n}+i / \tau_{l m n}$, $\omega_{l m n}=2 \pi f_{l m n}$ defining the angular frequency and $\tau_{l m n}$ the damping time. The ringdown wave will appear in a detector as the linear combination $h(t)$ of the two polarizations $h_{+}$and $h_{\times}$, that is $h(t)=F_{+} h_{+}+F_{\times} h_{\times}, F_{+}$and $F_{\times}$being the antenna pattern functions as defined in Equation (57). The polarization amplitudes for a given mode are given by

$$
\begin{aligned}
& h_{+}=\frac{A\left(f_{l m n}, Q_{l m n}, \epsilon_{\mathrm{rd}}\right)}{r}\left(1+\cos ^{2} \iota\right) \exp \left(\frac{-\pi f_{l m n} t}{Q_{l m n}}\right) \cos \left(2 \pi f_{l m n} t+\varphi_{l m n}\right), \\
& h_{\times}=\frac{A\left(f_{l m n}, Q_{l m n}, \epsilon_{\mathrm{rd}}\right)}{r} 2 \cos \iota \exp \left(\frac{-\pi f_{l m n} t}{Q_{l m n}}\right) \sin \left(2 \pi f_{l m n} t+\varphi_{l m n}\right),
\end{aligned}
$$

where $\iota$ is the angle between the black hole's spin axis and the observer's line of sight and $\varphi_{\operatorname{lmn}}$ is an unknown constant phase. The quality factor $Q_{l m n}$ of a mode is defined as $Q_{l m n}=\omega_{l m n} \tau_{l m n} / 2$ and gives roughly the number of oscillations that are observable before the mode dies out. Figure 8 [76] 
plots frequencies and quality factors for the first few QNMs as a function of the dimensionless spin parameter $j=J / M^{2}$. The mode of a Schwarzschild black hole corresponding to $l=2, m=n=0$, is given by

$$
f_{200}= \pm 1.207 \times 10^{3} \frac{10 M_{\odot}}{M} \mathrm{~Hz}, \quad \tau_{200}=5.537 \times 10^{-4} \frac{M}{10 M_{\odot}} \mathrm{s} .
$$

For stellar-mass-black-hole coalescences expected to be observed in ground-based detectors the ringdown signal is a transient that lasts for a very short time. However, for space-based LISA the signal would last several minutes for a black hole of $M=10^{7} M_{\odot}$. In the latter case, the ringdown waves could carry the energy equivalent of $10^{5} M_{\odot}$ converted to gravitational waves a phenomenal amount of energy compared even to the brightest quasars and gamma ray bursts. Thus, LISA should be able to see QNMs from black hole coalescences anywhere in the universe, provided the final (redshifted) mass of the black hole is larger than about $10^{6} M_{\odot}$, as otherwise the signal lasts for far too short a time for the detector to accumulate the SNR.

Berti et al. [76] have carried out an exhaustive study, in which they find that the LISA observations of SMBH binary mergers could be an excellent testbed for the no-hair theorem. Figure 9 (left panel) plots the fractional energy $\epsilon_{\text {rd }}$ that must be deposited in the ringdown mode so that the event is observable at a distance of $3 \mathrm{Gpc}$. Black holes at $3 \mathrm{Gpc}$ with mass $M$ in the range of $10^{6}-10^{8} M_{\odot}$ would be observable (i.e., will have an SNR of 10 or more) even if a fraction $\epsilon_{\mathrm{rd}} \simeq 10^{-7} M$ of energy is in the ringdown phase. Numerical relativity predicts that as much as $1 \%$ of the energy could be emitted as QNMs, when two black holes merge, implying that the ringdown phase could be observed with an SNR of 100 or greater all the way up to $z \sim 10$, provided their mass lies in the appropriate range ${ }^{10}$. Furthermore, they find that at this redshift it should be possible to resolve the fundamental $l=2, m=2$ mode. Since black holes forming from primordial gas clouds at $z=10-15$ could well be the seeds of galaxy formation and large-scale structure, LISA could indeed witness their formation through out the cosmic history of the universe.
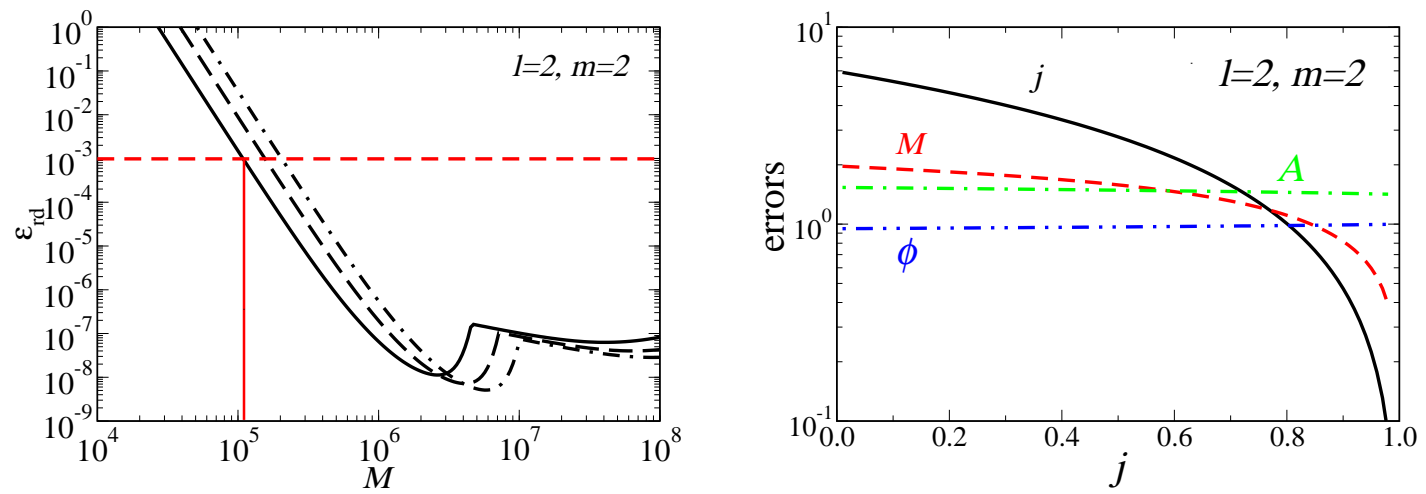

Figure 9: The smallest fraction of black hole mass in ringdown waveforms that is needed to observe the fundamental mode at a distance of $3 \mathrm{Gpc}$ (left) for three values of the black hole spin, $j=0$ (solid line) $j=0.80$ (dashed line) and $j=0.98$ (dot-dashed line) and the error in the measurement of the various parameters as a function of the black hole spin for the same mode (right). Figure reprinted with permission from [76]. (c) The American Physical Society.

Figure 9 (right panel) shows SNR-normalized errors (i.e., one-sigma deviations multiplied by

\footnotetext{
${ }^{10}$ Note that a black hole of physical mass $M$ at a redshift of $z$ will appear as a black hole of mass $M_{z}=(1+z) M$. This shifts the frequency of the QNM to the lower end of the spectrum. Assuming a frequency cutoff of $10^{-4} \mathrm{~Hz}$ for LISA, this means that only black holes of intrinsic mass $M<1.2 \times 10^{8} M_{\odot} /(1+z)$ can be observed at a redshift $z$.
} 
the SNR) in the measurement of the various QNM parameters (the mass of the hole $M$, its spin $j$, the QNM amplitude $A$ and phase $\varphi$ ) for the fundamental $l=m=2$ mode. We see that, for expected ringdown efficiencies of $\epsilon_{\mathrm{rd}} \simeq 10^{-2} M$ into the fundamental mode of an a-million-solarmass black hole with spin $j=0.8$ at $3 \mathrm{Gpc}(\rho \sim 2000)$, the mass and spin of the black hole can measured to an accuracy of a tenth of a percent.

By observing a mode's frequency and damping time, one can deduce the (redshifted) mass and spin of the black hole. However, this is not enough to test the no-hair theorem. It would be necessary, although by no means sufficient, to observe at least one other mode (whose damping time and frequency can again be used to find the black hole's mass and spin) to see if the two are consistent with each other. Berti et al [76] find that such a measurement should be possible if the event occurs within a redshift of $z \sim 0.5$.

\subsection{The two-body problem in general relativity}

The largest effort in gravitational radiation theory in recent years has been to study the two-body problem using various approximations. The reason is that gravitationally bound binary systems are likely to be important gravitational wave sources, and until the evolution of such a system is thoroughly understood, it will not be possible to extract the maximum possible information from the observations.

From Figure 2, we see that ground-based detectors will be sensitive to compact binaries with mass in the range of $\left[1,10^{4}\right] M_{\odot}$ while LISA will be sensitive to the mass range $\left[10^{4}, 10^{8}\right] M_{\odot}$. As we have seen in Section 3, most classes of binary sources will follow orbits that evolve strongly due to gravitational radiation reaction. In the case of ground-based detectors, they will all merge within a year of entering the observation band. In the case of LISA, we might observe sources (both stellar mass binaries as well as SMBH binaries), whose frequency hardly changes.

In contrast to Newtonian gravity, modeling a bound binary in general relativity is complicated by the existence of gravitational radiation and the nonlinearity of Einstein's equations. It must therefore be done approximately. The three most important approximation methods for solving gravitational wave problems are:

- The post-Newtonian scheme. This is a combination of a low-velocity expansion $(v / c$ small) and a weak-field expansion $(M / R$ small), in which the two small parameters are linked because a gravitationally-bound binary satisfies the virial relation $v^{2} \sim M / R$, even in relativity. The zero-order solution is the Newtonian binary system. The post-Newtonian $(\mathrm{PN})$ approximation has now been developed to a very high order in $v / c$ because the velocities in late-stage binaries, just before coalescence, are very high.

- Perturbation theory. This is an expansion in which the small parameter is the massratio of the binary components. The zero-order solution is the field of the more massive component, and linear field corrections due to the second component determine the binary's orbital motion and the emitted radiation. This approximation is fully relativistic at all orders. It is being used to study the signals emitted by compact stars and stellar-mass black holes as they fall into SMBHs, an important source for LISA.

- Numerical approaches. With numerical relativity one can in principle simulate any desired relativistic system, no matter how strong the fields or high the velocities. It is being used to study the final stage of the evolution of binaries, including their coalescence, after the PN approximation breaks down. Although it deals with fully relativistic and nonlinear general relativity, the method needs to be regarded as an approximate one, since spacetime is not resolved to infinite precision. The accuracy of a numerical simulation is normally judged by performing convergence tests, that is by doing the simulation at a variety of resolutions and showing that there are no unexpected differences between them.

Living Reviews in Relativity

http://www.livingreviews.org/lrr-2009-2 
We will review the physics that can be learned from models using each of these approximation schemes. But first we treat a subject that is common to all binaries that evolve due to radiation reaction, which is that one can estimate their distance from a gravitational wave observation.

\subsubsection{Binaries as standard candles: distance estimation}

Astronomers refer to systems as standard candles if their intrinsic luminosity is known, so that when the apparent luminosity of a particular system is measured, then its distance can be deduced. As mentioned in Section 3.4.2, radiating binaries have this property, if one can measure the effects of radiation reaction on their orbits [329]. Because of the one-dimensional nature of gravitational wave data, some scientists have begun calling these standard sirens [195]. Over cosmological distances, the distance measured from the observation is the luminosity distance. We discuss in Section 8 below how this can be used to determine the Hubble constant and even the acceleration of the universe in methods independent of any cosmic distance ladder.

\subsubsection{Numerical approaches to the two-body problem}

From the point of view of relativity, the simplest two-body problem is that of two black holes. There are no matter fields and no point particles, just pure gravity. Therefore, the physics is entirely governed by Einstein's equations, which are highly nonlinear and rather difficult to solve. A number of teams have worked for over three decades towards developing accurate numerical solutions for the coalescence of two black holes, using fully three-dimensional numerical simulations.

A breakthrough came in early 2005 with Pretorius [298] announcing the results from the first stable simulation ever, followed by further breakthroughs by two other groups [104, 61] with successful simulations. The main results from numerical simulations of nonspinning black holes are rather simple. Indeed, just as the EOB had predicted, and probably contrary to what many people had expected, the final merger is just a continuation of the adiabatic inspiral, leading on smoothly to merger and ringdown. In Figure 10 we show the results from one of the numerical simulations (right panel) and that of the EOB (left panel), both for the same initial conditions. There is also good agreement in the prediction of the total energy emitted by the system, being $5.0 \%( \pm 0.4 \%)$ (for a review see [299]) and 3.1\% [97], by numerical simulations and EOB, respectively, as well as the spin of the final black hole (respectively, 0.69 and 0.8 ) that results from the merger.

The total energy emitted and the spin angular momentum of the black hole both depend on the spin angular momenta of the parent black holes and how they are aligned with respect to the orbital angular momentum. In the test-mass limit, it is well known that the last stable orbit of a test particle in prograde orbit will be closer to, and that of a retrograde orbit will be farther from, the black hole as compared to the Schwarzschild case. Thus, prograde orbits last longer and radiate more compared to retrograde orbits. The same is true even in the case of spinning black holes of comparable masses; the emitted energy will be greater when the spins are aligned with the orbital angular momentum and least when they are anti-aligned. For instance, for two equal mass black holes, each with its spin angular momentum equal to 0.76 , the total energy radiated in the aligned (anti-aligned) case is $6.7 \%(2.2 \%)$ and the spin of the final black hole is $0.89(0.44)$ [105, 294]. Heuristically, in the aligned case the black holes experience a repulsive force, deferring the merger of the two bodies to a much later time than in the anti-aligned case, where they experience an attractive force, accelerating the merger.

Detailed comparisons $[137,284,88]$ show that we should be able to deploy the analytical templates from EOB $[100,135,138,139]$ (and other approximants [22]) that better fit the numerical data in our searches. With the availability of merger waveforms from numerical simulations and analytical templates, it will now be possible to search for compact binary coalescences with a greater sensitivity. The visibility of the signal improves significantly for binaries with their component masses in the range $[10,100] M_{\odot}$. Currently, an effort is underway to evaluate how to make use of 
numerical relativity simulations in gravitational wave searches [352], which should help to increase the distance reach of interferometric detectors by a factor of two and correspondingly nearly an order-of-magnitude increase in event rate.
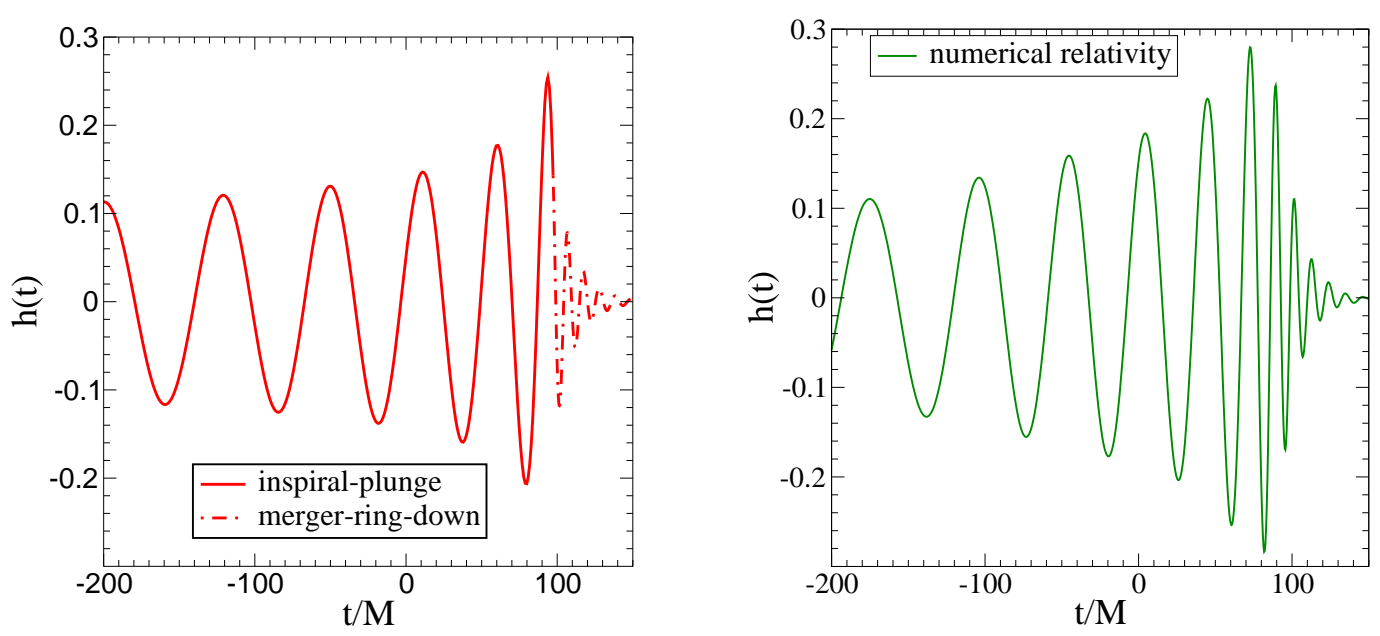

Figure 10: Comparison of waveforms from the analytical EOB approach (left) and numerical relativity simulations (right) for the same initial conditions. The two approaches predict very similar values for the total energy emitted in gravitational waves and the final spin of the black hole. Figure from [94].

Numerical relativity simulations have now greatly matured, allowing a variety of different studies. Some are studying the effect of the spin orientations of the component black holes on the linear momentum carried away by the final black hole, fancifully called kicks [188, 92, 62, 105, 180, 294]; some have focused on the dependence of the emitted waveform phase and energy on the mass ratio; and yet others have strived to evolve the system with high accuracy and for a greater number of cycles so as to push the techniques of numerical relativity to the limit $[89,88]$.

Of particular interest are the numerical values of black hole kicks that have been obtained for certain special configurations of the component spins. Velocities as large as $4000 \mathrm{~km} \mathrm{~s}^{-1}$ have been reported by several groups, but such velocities are only achieved when both black holes have large ${ }^{11}$ spins. Such velocities are in excess of escape velocities typical of normal galaxies and are, therefore, of great astronomical significance. These high velocities, however, are not seen for generic geometries of the initial spin orientations; therefore, their astronomical significance is not yet clear.

What is the physics behind kicks? Beamed emission of radiation from a binary could result in imparting a net linear momentum to the final black hole. The radiation could be beamed either because the masses of the two black holes are not the same (resulting in asymmetric emission in the orbital plane) or because of the precession of the orbital plane arising from spin-orbit and spin-spin interactions, or both. In the case of black holes with unequal masses, the largest kick one can get is around $170 \mathrm{~km} \mathrm{~s}^{-1}$, corresponding to a mass ratio of about $3: 1$. It was really with the advent of numerical simulations that superkicks begin to be realized, but only when black holes had large spins. The spin-orbit configurations that produce large kicks are rather unusual and at first sight unexpected. When the component black holes are both of the same mass and have equal but opposite spin angular momenta that lie in the orbital plane, frame dragging can lead to tilting and oscillation of the orbital plane, which, in the final phases of the evolution, could result in a rather large kick [299]. SMBHs are suspected to have large spins and, therefore, the effect of spin

\footnotetext{
${ }^{11} \mathrm{By}$ large spins we mean values that are close to the maximum value allowed by general relativity. If $\mathbf{J}$ is the magnitude of the spin angular momentum then general relativity requires that $|\mathbf{J}| \leq M^{2}$
} 
on the evolution of a binary and the final spin and kick velocity could be of astrophysical interest too.

Curiously, a recent optical observation of a distant quasar, SDSS J0927 12.65+294344.0, could well be the first identification of a superkick, causing the SMBH to escape from the parent galaxy [222]. From a fundamental physics point of view, kicks offer a new way of testing frame dragging in the vicinity of black holes, but much work is needed in this direction.

More recently, there has been an effort to understand and predict [98, 309, 136] the spin of the final black hole, which should help in further exploring interesting regions of the spin parameter space. In the relatively simple case of two black holes with equal and aligned spins of magnitude $a$, but unequal masses, with the symmetric mass ratio being $\nu=m_{1} m_{2} /\left(m_{1}+m_{2}\right)^{2}$, Rezzolla et al. [309] have obtained an excellent fit for the final spin $a_{\text {fin }}$ of the black hole by enforcing basic constraints from the test-mass limit:

$$
a_{\text {fin }}=a+\left(2 \sqrt{3}+t_{0} a+s_{4} a^{2}\right) \nu+\left(s_{5} a+t_{2}\right) \nu^{2}+t_{3} \nu^{3},
$$

where $t_{0}=-2.686 \pm 0.065, t_{2}=-3.454 \pm 0.132, t_{3}=2.353 \pm 0.548, s_{4}=-0.129 \pm 0.012$, and $s_{5}=-0.384 \pm 0.261$. The top and middle panels of Figure 11 compare as functions of black hole spin and the symmetric mass ratio the goodness of their fit (blue short-dashed line, top panels) with the predictions of numerical simulations (circles and stars) from different groups (AEI [311], FAU-Jena [251], Jena [75] and Goddard [100]). Their residuals (red dotted lines, bottom panels) are less than a percent over the entire parameter space observed. These figures also show the fits obtained for the equal-mass but variable-spin case (green long-dashed line, left panel) [98] and for the nonspinning but unequal-mass case (green long-dashed line, middle panel) [136].

For the simple case of two equal mass black holes with aligned spins, the above analytical formula predicts that minimal and maximal final spin values of $a_{\text {fin }}=0.35 \pm 0.03$ and $a_{\text {fin }}=$ $0.96 \pm 0.03$, respectively [309]. More interestingly, one can now ask what initial configurations of the mass ratios and spins would lead to the formation of a Schwarzschild black hole (i.e., $\left.a_{\text {fin }}(a, \nu)=0\right)$ [199], which defines the boundary of the region on one side of which lie systems for which the spin of the final black hole flips relative to the initial total angular momentum (bottom panel in Figure 11).

Finally, the evolution of binaries composed of nonspinning bodies is characterized by a single parameter, namely the ratio of the masses of the two black holes. The study of systems with different mass ratios has allowed relativists to fit numerical waveforms with phenomenological waveforms [22]. The advantage of the latter waveforms is that one is able to more readily carry out data analysis in any part of the parameter space without needing the numerical data over the entire signal manifold.

Numerical relativity is still in its infancy and the parameter space is quite large. In the coming years more accurate simulations should become available, allowing the computation of waveforms with more cycles and less systematic errors. However, the challenge remains to systematically explore the effect of different spin orientations, mass ratios and eccentricity. One area that has not been explored using perturbative methods or post-Newtonian theory is that of intermediatemass-ratio inspirals. These are systems with moderate mass ratios of order 100:1, where neither black-hole perturbation theory nor post-Newtonian approximation might be adequate. Yet, the prospect for detecting such systems in ground and space-based detectors is rather high. Numerical relativity simulations might be the only way to set up effectual search templates for such systems.

\subsubsection{Post-Newtonian approximation to the two-body problem}

For the interpretation of observations of neutron-star-binary coalescences, which might be detected within five years by upgraded detectors that are now taking data, it is necessary to understand their orbital evolution to a high order in the PN expansion. The first effects of radiation reaction 



Figure 11: The final spin of a black hole that results from the merger of two equal mass black holes of aligned spins (top panel) and nonspinning unequal mass black holes (middle panel). The bottom panel shows the region in the parameter space that results in an overall flip in the spin-orbit orientation of the system. Figure reprinted with permission from [310]. See text for details. (c) The American Astronomical Society. 
are seen at $2.5 \mathrm{PN}$ order (i.e., at order $(v / c)^{5}$ beyond Newtonian gravity), but we probably have to have control in the equations of motion over the expansion at least to $3.5 \mathrm{PN}$ order beyond the first radiation reaction (i.e., to order $(v / c)^{12}$ beyond Newtonian dynamics). There are many approaches to this, and we can not do justice here to the enormous effort that has gone into this field in recent years and refer the reader to the Living Reviews by Blanchet [79] and by Futamase \& Itoh [169].

Most work on this problem so far has treated a binary system as if it were composed of two point masses. This is, strictly speaking, inconsistent in general relativity, since the masses should form black holes of finite size. Blanchet, Damour, Iyer, and collaborators [78] have avoided this problem by a method that involves generalized functions. They first expand in the nonlinearity parameter, and, when they have reached sufficiently high order, they obtain the velocity expansion of each order. By ordering terms in the post-Newtonian manner they have developed step-by-step the approximations up to $3.5 \mathrm{PN}$ order.

A different team, led by Will, works with a different method of regularizing the point-particle singularity and compares its results with those of Blanchet et al. at each order [82]. There is no guarantee that either method can be continued successfully to any particular order, but so far they have worked well and are in agreement. Their results form the basis of the templates that are being designed to search for binary coalescences.

An interesting way of extending the validity of the expansion that is known to any order is to use Padé approximants [133, 134] (rational polynomials) of the fundamental quantities in the theory, namely the orbital energy and the gravitational wave luminosity. This has worked rather well in improving the convergence of PN theory. Buonanno and Damour $[96,97]$ have proposed an EOB approach to two-body dynamics, which makes it possible to compute the orbit of the binary and hence the phasing of the gravitational waves emitted beyond the last stable orbit into the merger and ringdown phases in the evolution of the black hole binary. This analytical approach has been remarkably successful and gained a lot of ground after the recent success in numerical relativity (see Section 6.5.2).

Other methods have been applied to this problem. Futamase [168] introduced a limit that combines the nonlinearity and velocity expansions in different ways in different regions of space, so that the orbiting bodies themselves have a regular (finite relativistic self-gravity) limit, while their orbital motion is treated in a Newtonian limit. This should not fail at any order [169], and has demonstrated its robustness by arriving at the same results as the other approaches, at least through $3 \mathrm{PN}$ order. But it has a degree of arbitrariness in choosing initial data (see [327]) that could cause problems for gravitational wave search templates that integrate orbits for a long period of time.

Linear calculations of point particles around black holes are of interest in themselves and also for checking results of the full two-body calculations. These are well-developed for certain situations, e.g., [354, 258]. But the general equation of motion for such a body, taking into account all nongeodesic effects, has not yet been cast into a form suitable for practical calculations [106, 301]. This field is reviewed by two separate Living Reviews [292, 319].

Matched filtering, discussed in Section 5.1, is a plausible method of testing the validity of different approaches to computing the inspiral and merger waveforms from binary systems. Though a single observation is not likely to settle the question as to which methods are correct, a catalogue of events will help to evaluate the accuracy of different approaches by studying the statistics of the SNRs they measure.

6.5.3.1 Post-Newtonian expansions of energy and luminosity. Post-Newtonian calculations yield the expansion of the gravitational binding energy $E$ and the gravitational wave lu- 
minosity $\mathcal{F}$ as a function of the post-Newtonian expansion parameter ${ }^{12} v$. This is related to the frequency $f_{\mathrm{gw}}$ of the dominant component of gravitational waves emitted by the binary system by

$$
v^{3}=\pi M f_{\mathrm{gw}}
$$

where $M$ is the total mass of the system. The expansions for a circular binary are $[80,81,79]$

$$
\begin{aligned}
E & =-\frac{\nu M v^{2}}{2}\left\{1+\left(-\frac{9+\nu}{12}\right) v^{2}+\left(\frac{-81+57 \nu-\nu^{2}}{24}\right) v^{4}\right. \\
& \left.+\left(-\frac{675}{64}+\left[\frac{34445}{576}-\frac{205 \pi^{2}}{96}\right] \nu-\frac{155}{96} \nu^{2}-\frac{35}{5184} \nu^{3}\right) v^{6}+\mathcal{O}\left(v^{8}\right)\right\},
\end{aligned}
$$

and

$$
\begin{aligned}
\mathcal{F} & =\frac{32 \nu^{2} v^{10}}{5}\left\{1-\left(\frac{1247}{336}+\frac{35}{12} \nu\right) v^{2}+4 \pi v^{3}+\left(-\frac{44711}{9072}+\frac{9271}{504} \nu+\frac{65}{18} \nu^{2}\right) v^{5}\right. \\
& -\left(\frac{8191}{672}+\frac{583}{24}\right) \pi v^{5}+\left[\frac{6643739519}{69854400}+\frac{16}{3} \pi^{2}-\frac{1712}{105}(\gamma+\ln (4 v))\right. \\
& \left.+\left(-\frac{4709005}{272160}+\frac{41}{48} \pi^{2}\right) \nu-\frac{94403}{3024} \nu^{2}-\frac{775}{324} \nu^{3}\right] v^{6} \\
& \left.+\left(-\frac{16285}{504}+\frac{214745}{1728} \nu+\frac{193385}{3024} \nu^{2}\right) \pi v^{7}+\mathcal{O}\left(v^{8}\right)\right\}
\end{aligned}
$$

where $\gamma=0.577 \ldots$ is Euler's constant.

6.5.3.2 Evolution equation for the orbital phase. Starting from these expressions, one requires that gravitational radiation comes at the expense of the binding energy of the system (see, e.g., [134]):

$$
\mathcal{F}=-\frac{d E}{d t}
$$

the energy balance equation. This can then be used to compute the (adiabatic) evolution of the various quantities as a function of time. For instance, the rate of change of the orbital velocity $\omega(t)=v^{3} / M$ ( $M$ being the total mass) can be computed using:

$$
\frac{d \omega(t)}{d t}=\frac{d \omega}{d v} \frac{d v}{d E} \frac{d E}{d t}=\frac{3 v^{2}}{M} \frac{\mathcal{F}(v)}{E^{\prime}(v)}, \quad \frac{d v}{d t}=\frac{d v}{d E} \frac{d E}{d t}=\frac{-\mathcal{F}(v)}{E^{\prime}(v)},
$$

where $E^{\prime}(v)=d E / d v$. Supplemented with a differential equation for $t$,

$$
d t=\frac{d t}{d E} \frac{d E}{d v}=-\frac{E^{\prime}(v)}{\mathcal{F}},
$$

one can solve for the evolution of the system's orbital velocity. Similarly, the evolution of the orbital phase $\varphi(t)$ can be computed using

$$
\frac{d \varphi(t)}{d t}=\frac{v^{3}}{M}, \quad \frac{d v}{d t}=\frac{-\mathcal{F}(v)}{E^{\prime}(v)} .
$$

\footnotetext{
${ }^{12}$ In Newton's theory a two-body problem can be reduced to a one-body problem, in which a body of reduced mass $\mu$ moves in an effective potential. The parameter $v$ is the velocity of the reduced mass, if the orbit is circular. In the extreme mass ratio limit $\nu \rightarrow 0, v$ is the velocity of the smaller mass.
} 
6.5.3.3 Phasing formulas. The foregoing evolution equations for the orbital phase can be solved in several equivalent ways [134], each correct to the required post-Newtonian order, but numerically different from one another. For instance, one can retain the rational polynomial $\mathcal{F}(v) / E(v)$ in Equation (118) and solve the two differential equations numerically, thereby obtaining the time evolution of $\varphi(t)$. Alternatively, one might re-expand the rational function $\mathcal{F}(v) / E(v)$ as a polynomial in $v$, truncate it to order $v^{n}$ (where $n$ is the order to which the luminosity is given), thereby obtaining a parametric representation of the phasing formula in terms of polynomial expressions in $v$ :

$$
\varphi(v)=\varphi_{\mathrm{ref}}+\sum_{k=0}^{n} \varphi_{k} v^{k}, \quad t(v)=t_{\mathrm{ref}}+\sum_{k=0}^{n} t_{k} v^{k},
$$

where $\varphi_{\text {ref }}$ and $t_{\text {ref }}$ are a reference phase and time, respectively. The standard post-Newtonian phasing formula goes one step further and inverts the second of the relations above to express $v$ as a polynomial in $t$ (again truncated to appropriate order), which is then substituted in the first of the expressions above to obtain a phasing formula as an explicit function of time:

$$
\begin{aligned}
\varphi(t)= & \frac{-1}{\nu \tau^{5}}\left\{1+\left(\frac{3715}{8064}+\frac{55}{96} \nu\right) \tau^{2}-\frac{3 \pi}{4} \tau^{3}+\left(\frac{9275495}{14450688}+\frac{284875}{258048} \nu+\frac{1855}{2048} \nu^{2}\right) \tau^{4}\right. \\
+ & \left(-\frac{38645}{172032}+\frac{65}{2048} \nu\right) \pi \tau^{5} \ln \tau+\left[\frac{831032450749357}{57682522275840}-\frac{53}{40} \pi^{2}-\frac{107}{56}(\gamma+\ln (2 \tau))\right. \\
+ & \left.\left(-\frac{126510089885}{4161798144}+\frac{2255}{2048} \pi^{2}\right) \nu+\frac{154565}{1835008} \nu^{2}-\frac{1179625}{1769472} \nu^{3}\right] \tau^{6} \\
+ & \left.\left(\frac{188516689}{173408256}+\frac{488825}{516096} \nu-\frac{141769}{516096} \nu^{2}\right) \pi \tau^{7}\right\} \\
v^{2} & =\frac{\tau^{2}}{4}\left\{1+\left(\frac{743}{4032}+\frac{11}{48} \nu\right) \tau^{2}-\frac{\pi}{5} \tau^{3}+\left(\frac{19583}{254016}+\frac{24401}{193536} \nu+\frac{31}{288} \nu^{2}\right) \tau^{4}\right. \\
& +\left(-\frac{11891}{53760}+\frac{109}{1920} \nu\right) \pi \tau^{5}+\left[-\frac{10052469856691}{6008596070400}+\frac{\pi^{2}}{6}+\frac{107}{420}(\gamma+\ln 2 \tau)\right. \\
& \left.+\left(\frac{3147553127}{780337152}-\frac{451}{3072} \pi^{2}\right) \nu-\frac{15211}{442368} \nu^{2}+\frac{25565}{331776} \nu^{3}\right] \tau^{6} \\
& \left.+\left(-\frac{113868647}{433520640}-\frac{31821}{143360} \nu+\frac{294941}{3870720} \nu^{2}\right) \pi \tau^{7}\right\}
\end{aligned}
$$

In the above formulas $v=\pi M f_{\mathrm{gw}}$ and $\tau=\left[\nu\left(t_{C}-t\right) /(5 M)\right]^{-1 / 8}, t_{C}$ being the time at which the two stars merge together and the gravitational wave frequency $f_{\mathrm{gw}}$ formally diverges.

6.5.3.4 Waveform polarizations. The post-Newtonian formalism also gives the two polarizations $h_{+}$and $h_{\times}$as multipole expansions in powers of the parameter $v$. To lowest order, the two polarizations of the radiation from a binary with a circular orbit, located at a distance $D$, with total mass $M$ and symmetric mass ratio $\nu=m_{1} m_{2} / M^{2}$, are given by

$$
h_{+}=\frac{2 \nu M}{D} v^{2}\left(1+\cos ^{2} \iota\right) \cos [2 \varphi(t)], \quad h_{\times}=\frac{4 \nu M}{D} v^{2} \cos \iota \sin [2 \varphi(t)],
$$

where $\iota$ is the inclination of the orbital plane with the line of sight and $v$ is the velocity parameter introduced earlier.

An interferometer will record a certain combination of the two polarizations given by

$$
h(t)=F_{+} h_{+}+F_{\times} h_{\times},
$$


where the beam pattern functions $F_{+}$and $F_{\times}$are those discussed in Section 4.2.1. In the case of ground-based instruments, the signal duration is pretty small, at most 15 min for neutron star binaries and smaller for heavier systems. Consequently, one can assume the source direction to be unchanging during the course of observation and the above combination produces essentially the same functional form of the waveforms as in Equation (122). Indeed, it is quite straightforward to show that

$$
h(t)=4 \nu M \frac{\mathcal{C}}{D} v^{2} \cos \left[2 \varphi(t)+2 \varphi_{0}\right]
$$

where

$$
\mathcal{C}=\sqrt{A^{2}+B^{2}}, \quad A=\frac{1}{2}\left(1+\cos ^{2} \iota\right) F_{+}, \quad B=\cos \iota F_{\times}, \quad \tan 2 \varphi_{0}=\frac{B}{A} .
$$

The factor $\mathcal{C}$ is a function of the various angles and lies in the range $[0,1]$ with an RMS value of $2 / 5$ (see Section 4.2.1, especially the discussion following Equation (62)).

These waveforms form the basis for evaluating the science that can be extracted from future observations of neutron star and black hole binaries. We will discuss the astrophysical and cosmological measurements that are made possible with such high precision waveforms in several sections that follow (6.5.5 and 8.3). It is clear from the expressions for the waveform polarizations that, at the lowest order, the radiation from a binary is predominantly emitted at twice the orbital frequency. However, even in the case of quasi-circular orbits the waves come off at other harmonics of the orbital frequency. As we shall see below, these harmonics are very important for estimating the parameters of a binary, although they do not seem to contribute much to the SNR of the system.

\subsubsection{Measuring the parameters of an inspiraling binary}

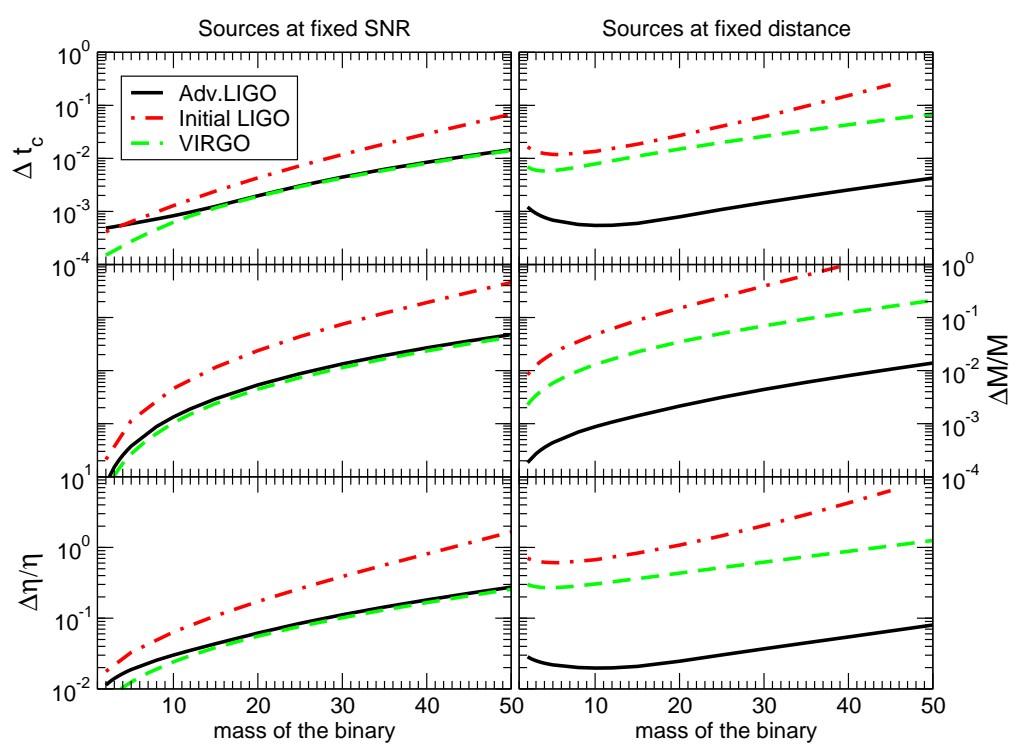

Figure 12: One-sigma errors in the time of coalescence, chirpmass and symmetric mass ratio for sources with a fixed SNR (left panels) and at a fixed distance (right panels). The errors in the time of coalescence are given in ms, while in the case of chirpmass and symmetric mass ratio they are fractional errors. These plots are for nonspinning black hole binaries; the errors reduce greatly when dynamical evolution of spins are included in the computation of the covariance matrix. Slightly modified figure from [49]. 
The issue of parameter estimation in the context of black hole binaries has received a lot of attention [114, 128, 165, 64, 293, 65, 44]. Most authors have used the covariance matrix for this purpose, although Markov Chain Monte Carlo (MCMC) techniques have also been used occasionally $[116,316,315,120]$, especially in the context of LISA [364, 121, 122, 123, 125]. Covariance matrix is often the preferred method, as one can explore a large parameter space without having to do expensive Monte Carlo simulations. However, when the parameter space is large, covariance matrix is not a reliable method for estimating parameter accuracies, especially at low SNRs [63, 64, 372]; but at high SNRs, as in the case of SMBH binaries in LISA, the problem might be that our waveforms are not accurate enough to facilitate a reliable extraction of the source parameters [129]. Although MCMC methods can give more reliable estimates, they suffer from being computationally extremely expensive. However, they are important in ascertaining the validity of results based on the covariance matrix, at least in a small subset of the parameter space, and should probably be employed in assessing parameter accuracies of candidate gravitational wave events.

In what follows we shall summarize the most recent work on parameter estimation in ground and space-based detectors for binaries with and without spin and the improvements brought about by including higher harmonics.

6.5.4.1 Ground-based detectors - nonspinning components. In Figure 12 we have plotted the one-sigma uncertainty in the measurement of the time of coalescence, chirpmass and symmetric mass ratio for initial and advanced LIGO and VIRGO [44]. The plots show errors for sources all producing a fixed SNR of 10 (left panels) or all at a fixed distance of $300 \mathrm{Mpc}$ (right panels). The fractional error in chirpmass, even at a modest SNR of 10, can be as low as a few parts in ten thousand for stellar mass binaries, but the error stays around $1 \%$, even for heavier systems that have only a few cycles in a detector's sensitivity band. Error in the mass ratio is not as small, increasing to $100 \%$ at the higher end of the mass range explored. Thus, although the chirpmass can be measured to a good accuracy, poor estimation of the mass ratio means that the individual masses of the binary cannot be measured very well. Note also that the time of coalescence of the signal is determined pretty well, which means that we would be able to measure the location of the system in the sky quite well.

At a given SNR the accuracy is better in the case of low-mass binaries, since they spend a longer duration and a greater number of cycles in the detector band and the chirpmass can be determined better than the mass ratio, since to first order the frequency evolution of a binary is determined only by the chirpmass.

6.5.4.2 Measuring the parameters of supermassive black hole binaries in LISA. In the case of LISA, the merger of SMBHs produces events with extremely large SNRs, even at a redshift of $z=1$ (100s to several thousands depending on the chirpmass of the source). Therefore, one expects to measure the parameters of a merger event in LISA to a phenomenal accuracy. Figure 13 depicts the distribution of the errors for a binary consisting of two SMBHs of masses $\left(10^{6}, 3 \times 10^{5}\right) M_{\odot}$ at a redshift of $z=1$ [232]. The distribution was obtained for ten thousand samples of the system corresponding to random orientations of the binary at random sky locations with the starting frequency greater than $3 \times 10^{-5} \mathrm{~Hz}$ and the ending frequency corresponding to the last stable orbit.

Each plot in Figure 13 shows the results of computations for binaries consisting of black holes with and without spins. Even in the absence of spin-induced modulations in the waveform, the parameter accuracies are pretty good. Note that spin-induced modulations in the waveform enable a far better estimation of parameters, chirpmass accuracy improving by more than an order of magnitude and reduced mass accuracy by two orders of magnitude. It is because of such accurate 

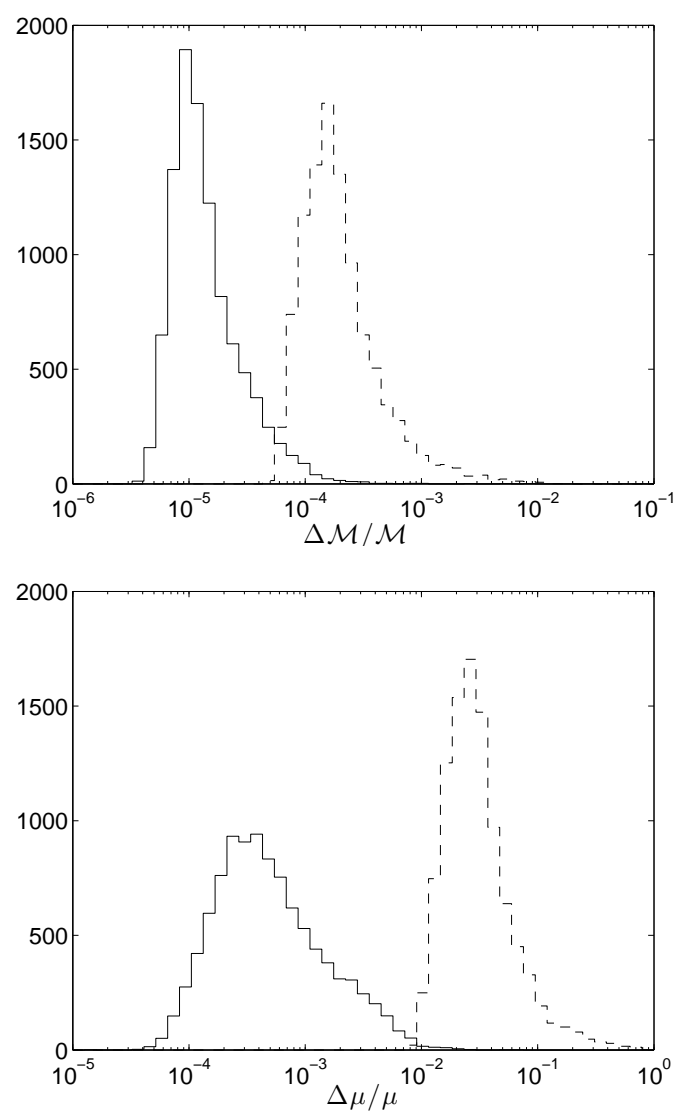

Figure 13: Distribution of measurement accuracy for a binary merger consisting of two black holes of masses $m_{1}=10^{6} M_{\odot}$ and $m_{2}=3 \times 10^{5} M_{\odot}$, based on 10,000 samples of the system in which the sky location and orientation of the binary are chosen randomly. Dashed lines are for nonspinning systems and solid lines are for systems with spin. Figure reprinted with permission from [231]. (C) The American Physical Society. 
measurements that it will be possible to use SMBH mergers to test general relativity in the strong field regime of the theory (see below).

Although Figure 13 corresponds to a binary with specific masses, the trends shown are found to be true more generically for other systems too, the actual parameter accuracies and improvements due to spin both depending on the specific system studied.

\subsubsection{Improvement from higher harmonics}
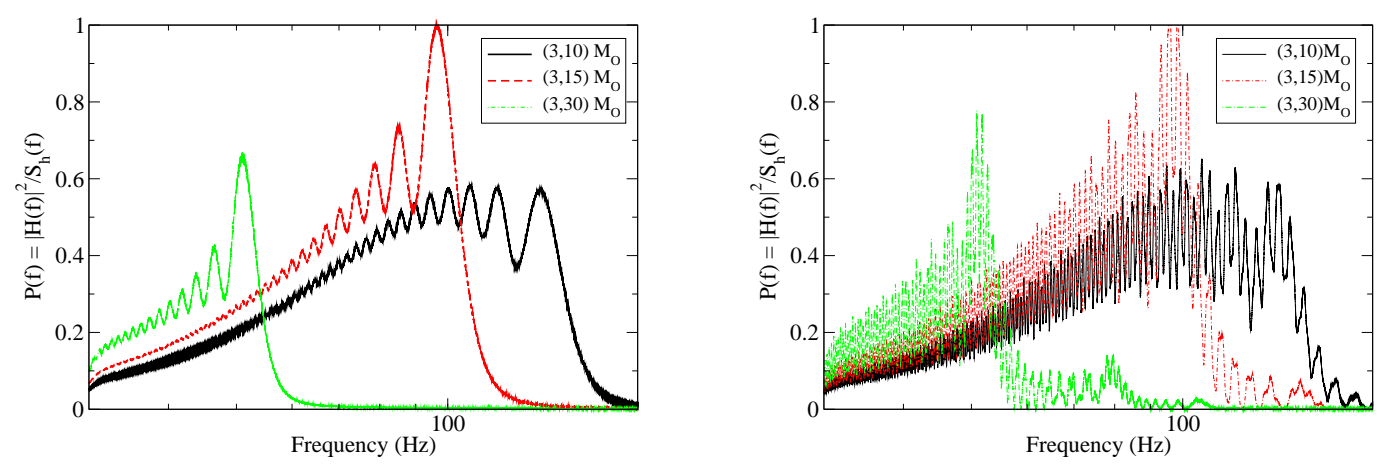

Figure 14: The SNR integrand of a restricted (left panel) and full waveform (right panel) as seen in initial LIGO. We have shown three systems, in which the smaller body's mass is the same, to illustrate the effect of the mass ratio. In all cases the system is at $100 \mathrm{Mpc}$ and the binary's orbit is oriented at $45^{\circ}$ with respect to the line of sight.

The results discussed so far use the restricted post-Newtonian approximation in which the waveform polarizations contain only twice the orbital frequency, neglecting all higher-order corrections (including those to the second harmonic). The full waveform is a post-Newtonian expansion of the two polarizations as a power-series in $v / c$ and consists of terms that have not only the dominant harmonic at twice the orbital frequency, but also other harmonics of the waveform. Schematically, the full waveform can be written as [79, 374]

$$
h(t)=\frac{4 M \eta}{D_{L}} \sum_{k=1}^{7} \sum_{n=0}^{5} A_{(k, n / 2)} v^{n+2}(t) \cos \left[k \varphi(t)+\varphi_{(k, n / 2)}\right],
$$

where $\nu=m_{1} m_{2} / M^{2}$ is the symmetric mass ratio, the first sum (index $k$ ) is over the different harmonics of the waveform and the second sum (index $n$ ) is over the different post-Newtonian orders. Note that post-Newtonian order weighs down the importance of higher-order amplitude corrections by an appropriate factor of the small parameter $v$. In the restricted post-Newtonian approximation one keeps only the lowest-order term. Since $A_{1,0}$ happens to be zero, the dominant term corresponds to $k=2$ and $n=0$, containing twice the orbital frequency.

The various signal harmonics, and the associated additional structure in the waveform, can potentially enhance our ability to measure the parameters of a binary to a greater accuracy. The reason we can expect to do so can be seen by looking at the spectra of gravitational waves with and without these harmonics. For a binary that is oriented face on with respect to a detector only the second harmonic is seen, while for any other orientation the radiation is emitted at all other harmonics, the influence of the harmonics becoming more pronounced as the inclination angle changes from 0 to $\pi / 2$. Figure 14 compares, in the frequency band of ground-based detectors, the spectrum of a source using the restricted post-Newtonian approximation (left panel) to the full waveform. In both cases the source is inclined to the line of sight at 45 degrees. 
Following is a list of improvements brought about by higher harmonics. In the case of groundbased detectors Van Den Broeck and Sengupta [374, 375] found that, when harmonics are included, the SNR hardly changes, but is always smaller, relative to a restricted waveform. However, the presence of frequencies higher than twice the orbital frequency means that it will be possible to observe heavier systems, increasing the mass reach of ground-based detectors by a factor of 2 to 3 in advanced LIGO and third generation detectors [374, 375]. The same effect was found in the case of LISA too, allowing LISA to observe SMBH masses up to a few $\times 10^{8} M_{\odot}$ [47]. More than the increased mass reach, the harmonics reduce the error in the estimation of the chirpmass, symmetric mass ratio and the time of arrival by more than an order of magnitude for stellar-mass black hole binaries. The same is true to a greater extent in the case of SMBH binaries, allowing as well a far greater accuracy in the measurement of the luminosity distance and sky resolution in LISA's observation of these sources [48, 361]. For instance, Figure 15 [361] shows the gain in LISA's angular resolution for two massive black-hole-binary mergers as a consequence of using higher harmonics for a specific orientation of the binary. Improvements of order 10 to 100 can be seen over large regions of the sky. This improved performance of LISA makes it a good probe of dark energy [48] (see Section 8.3).

A word of caution is in order with regard to the improvements brought about by higher harmonics. If the sensitivity of a detector has an abrupt lower frequency cutoff, or falls off rapidly below a certain frequency, then the harmonics bring about a more dramatic improvement than when the sensitivity falls off gently. Higher harmonics, nevertheless, always help in reducing the random errors associated with the measurement of parameters of a coalescing black-hole binary.

\subsection{Tests of general relativity}

Gravitational wave measurements of black holes automatically test general relativity in its strongfield regime. Observations of the mergers of comparable-mass black holes will be rich in details of their strong-field interactions. If measurements can determine the masses and spins of the initial black holes, as well as the eccentricity and orientation of their inspiral orbit, then one would hope to compare the actual observed waveform with the output of a numerical simulation of the same system. If measurements can also determine the final mass and spin (say from the ringdown radiation) then one can test the Hawking area theorem (the final area must exceed the sum of the areas of the initial holes) and the Penrose cosmic censorship conjecture (the final black hole should have $\left.J / M^{2}<1\right)$.

Observations of stellar mass black holes inspiraling into SMBHs, the extreme mass ratio inspirals (EMRIs), have an even greater potential for testing general relativity. The stellar mass black hole spends thousands of precessing (both of periastron and the orbital plane) orbits along highlyeccentric trajectories and slowly inspirals into the larger black hole. The emitted gravitational radiation literally carries the signature of the spacetime geometry around the central object. So fitting the orbit to theoretical templates could reveal small deviations of this geometry from that of Kerr. For example, if we know (from fitting the waveform) the mass and spin of the central black hole, then all its higher multipole moments are determined. If we can measure some of these and they deviate from Kerr, then that would indicate that either the central object is not a black hole or that general relativity needs to be corrected [177, 68].

\subsubsection{Testing the post-Newtonian approximation}

Current tests of general relativity rely on experiments in the solar system (using the sun's gravitational field) and observations of binary pulsars. In dimensionless units, the gravitational potential on the surface of the sun is about one part in a million and even in a binary pulsar the potential that each neutron star experiences due to its companion is no more than one part in ten thou-

Living Reviews in Relativity

http: //www. livingreviews.org/lrr-2009-2 

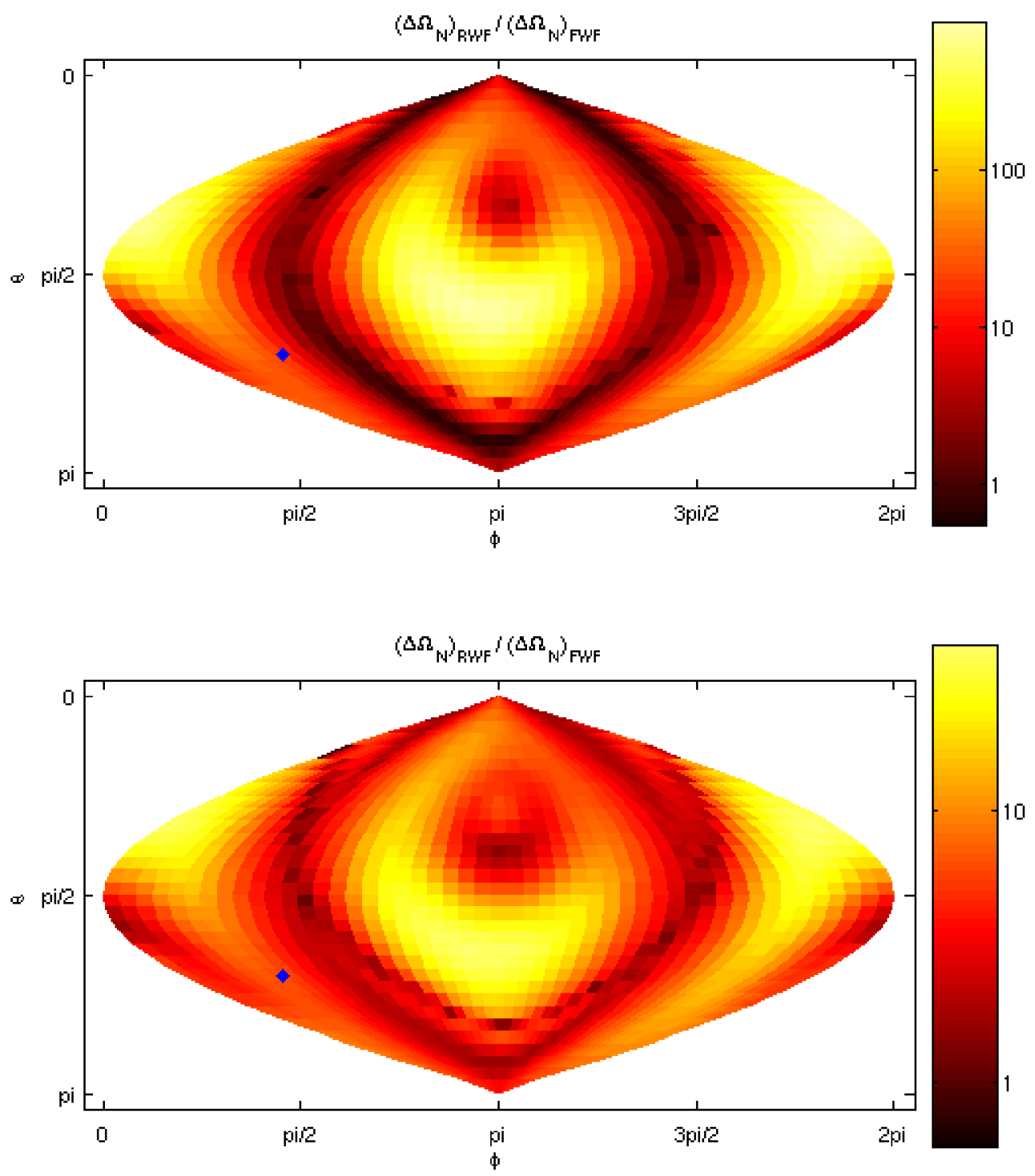

Figure 15: Sky map of the gain in angular resolution for LISA observations of the final year of inspirals using full waveforms with harmonics versus restricted post-Newtonian waveforms with only the dominant harmonic, corresponding to the equal mass case $\left(m_{1}=m_{2}=10^{7} M_{\odot}\right.$, top) and a system with mass ratio of $10\left(m_{1}=10^{7} M_{\odot}, m_{2}=10^{6} M_{\odot}\right.$, bottom). The sources are all at $z=1$, have the same orientation $\left(\cos \theta_{L}=0.2, \phi_{L}=3\right)$ and zero spins $\beta=\sigma=0$. Figure reprinted with permission from [361]. (C) The American Physical Society. 
sand. These are mildly relativistic fields, with the corresponding escape velocity being as large as a thousandth and a hundredth that of light, respectively.

Thus, gravitational fields in the solar system or in a binary pulsar are still weak by comparison to the largest possible values. Indeed, close to the event horizon of a black hole, gravitational fields can get as strong as they can ever get, with the dimensionless potential being of order unity and the escape velocity equal to that of the speed of light. Although general relativity has been found to be consistent with experiments in the solar system and observations of binary pulsars, phenomena close to the event horizons of black holes would be a great challenge to the theory. It would be very exciting to test Einstein's gravity under such circumstances.

The large SNR that is expected from SMBH binaries makes it possible to test Einstein's theory under extreme conditions of gravity [45, 46]. To see how one might test the post-Newtonian structure of Einstein's theory, let us consider the waveform from a binary in the frequency domain. Since an inspiral wave's frequency changes rather slowly (adiabatic evolution) it is possible to apply a stationary phase approximation to compute the Fourier transform $H(f)$ of the waveform given in Equation (124):

$$
H(f)=\mathcal{A} f^{-7 / 6} \exp \left[i \Psi(f)+i \frac{\pi}{4}\right]
$$

with the Fourier amplitude $\mathcal{A}$ and phase $\Psi(f)$ given by

$$
\mathcal{A}=\frac{\mathcal{C}}{D \pi^{2 / 3}} \sqrt{\frac{5 \nu}{24}} M^{5 / 6}, \quad \Psi(f)=2 \pi f t_{C}+\Phi_{C}+\frac{3}{128 \nu} \sum_{k} \alpha_{k}(\pi M f)^{(k-5) / 3} .
$$

Here $\nu$ is the symmetric mass ratio defined before (see Equation 31), $\mathcal{C}$ is a function of the various angles, as in Equation (124), and $t_{C}$ and $\Phi_{C}$ are the fiducial epoch of merger and the phase of the signal at that epoch, respectively. The coefficients in the PN expansion of the Fourier phase are given by

$$
\begin{aligned}
\alpha_{0} & =1, \quad \alpha_{1}=0, \quad \alpha_{2}=\frac{3715}{756}+\frac{55}{9} \nu, \quad \alpha_{3}=-16 \pi \\
\alpha_{4} & =\frac{15293365}{508032}+\frac{27145}{504} \nu+\frac{3085}{72} \nu^{2}, \quad \alpha_{5}=\pi\left(\frac{38645}{756}-\frac{65}{9} \nu\right)\left[1+\ln \left(6^{3 / 2} \pi M f\right)\right], \\
\alpha_{6} & =\frac{11583231236531}{4694215680}-\frac{640}{3} \pi^{2}-\frac{6848}{21} \gamma+\left(-\frac{15737765635}{3048192}+\frac{2255}{12} \pi^{2}\right) \nu \\
& +\frac{76055}{1728} \nu^{2}-\frac{127825}{1296} \nu^{3}-\frac{6848}{63} \ln (64 \pi M f), \\
\alpha_{7} & =\pi\left(\frac{77096675}{254016}+\frac{378515}{1512} \nu-\frac{74045}{756} \nu^{2}\right) .
\end{aligned}
$$

These are the PN coefficients in Einstein's theory; in an alternative theory of gravity they will be different. In Einstein's theory the coefficients depend only on the two mass parameters, the total mass $M$ and symmetric mass ratio $\nu$. One of the tests we will discuss below concerns the consistency of the various coefficients. Note, in particular, that in Einstein's gravity the 0.5 PN term is absent, i.e., the coefficient of the term $v$ is zero. Even with the very first observations of inspiral events, it should be possible to test if this is really so.

Figure 16 shows one such test that is possible with SMBH binaries [45, 46]. The observation of these systems in LISA makes it possible to measure the parameters associated with different physical effects. For example, the rate at which a signal chirps (i.e., the rate at which its frequency changes) depends on the binary's chirpmass. Given the chirpmass, the length of the signal depends on the system's symmetric mass ratio (the ratio of reduced mass to total mass). Another example would be the scattering of gravitational waves off the curved spacetime geometry of the binary,

Living Reviews in Relativity

http://www. livingreviews.org/lrr-2009-2 
producing the tail effect in the emitted signal, which is determined principally by the system's total mass [85, 84]. Similarly, spin-orbit interaction, spin-spin coupling, etc. depend on other combinations of the masses.

The binary will be seen with a high SNR, which means that we can measure the mass parameters associated with many of these physical effects. If each parameter is known precisely, we can draw a curve corresponding to it in the space of masses. However, our observations are inevitably subject to statistical (and possibly systematic) errors. Therefore, each parameter corresponds to a region in the parameter space (shown in Figure 16 for the statistical errors only). If Einstein's theory of gravitation is correct, the regions corresponding to the different parameters must all have at least one common region, a region that contains the true parameters of the binary. This is because Einstein's theory, or an alternative, has to be used to project the observed data onto the space of masses. If the region corresponding to one or more of these parameters does not overlap with the common region of the rest of the parameters, then Einstein's theory, or its alternative, is in trouble.

In Brans-Dicke theory the system is expected to emit dipole radiation and the PN series would begin an order $v^{-2}$ earlier than in Einstein's theory. In the notation introduced above we would have an $\alpha_{-2}$ term, which has the form $[73,74]$

$$
\alpha_{-2}=-\frac{5 \mathcal{S}^{2}}{84 \omega_{\mathrm{BD}}} .
$$

Here $\mathcal{S}$ is the the difference in the scalar charges of the two bodies and $\omega_{\mathrm{BD}}$ is the Brans-Dicke parameter. Although this term is formally two orders lower than the lowest-order quadrupole term of Einstein's gravity (i.e., it is $\mathcal{O}\left(v^{-2}\right)$ order smaller), numerically its effect will be far smaller than the quadrupole term because of the rather large bound on $\omega_{\mathrm{BD}} \gg 1$. Nevertheless, its importance lies in the fact that there is now a new parameter on which the phase depends. Berti, Buonanno and Will conclude that LISA observations of massive black-hole binaries will enable scientists to set limits on $\omega_{\mathrm{BD}} \sim 10^{4}-10^{5}$.

A massive graviton theory would also alter the phase. The dominant effect is at $1 \mathrm{PN}$ order and would change the coefficient $\alpha_{2}$ to

$$
\alpha_{2} \rightarrow \alpha_{2}-\frac{128 \nu}{3} \frac{\pi^{2} D M}{\lambda_{g}^{2}(1+z)},
$$

where $\nu$ is the symmetric mass ratio. This term alters the time of arrival of waves of different frequencies, causing a dispersion, and a corresponding modulation, in the wave's phase, depending on the Compton wavelength $\lambda_{g}$ and the distance $D$ to the binary. Hence, by tracking the phase of the inspiral waves, one can bound the graviton's mass. Will [393] finds that one can bound the mass to $1.7 \times 10^{13} \mathrm{~km}$ using ground-based detectors and $1.7 \times 10^{17} \mathrm{~km}$ using space-based detectors, as also confirmed by more recent and exhaustive calculations [73]. These limits might improve if one takes into account the modulation of the waveform due to spin-orbit and spin-spin coupling, but the latter authors [73] looked at spinning, but nonprecessing, systems only.

\subsubsection{Uniqueness of Kerr geometry}

In Section 3 we pointed out that LISA should be able to see many hundreds of signals emitted by compact objects - black holes, neutron stars, even white dwarfs - orbiting around and being captured by massive black holes in the centers of galaxies. But for LISA to reach its full potential, we must model the orbits and their emitted radiation accurately. Since the wave trains may be many hundreds or thousands of cycles long in the LISA band, the challenge of constructing template waveforms that remain accurate to within about one radian over the whole evolution is significant. 



Figure 16: By fitting the Fourier transform of an observed signal to a post-Newtonian expansion, one can measure the various post-Newtonian coefficients $\psi_{k}\left(m_{1}, m_{2}\right), k=0,2,3,4,6,7$ and coefficients of log-terms $\psi_{5 l}\left(m_{1}, m_{2}\right)$ and $\psi_{6 l}\left(m_{1}, m_{2}\right)$. In Einstein's theory, all the coefficients depend only on the two masses of the component black holes. By treating them as independent parameters one affords a test of the post-Newtonian theory. Given a measured value of a coefficient, one can draw a curve in the $m_{1}-$ $m_{2}$ plane. If Einstein's theory is correct, then the different curves must all intersect at one point within the allowed errors. These plots show what might be possible with LISA's observation of the merger of a binary consisting of a pair of $10^{6} M_{\odot}$ black holes. In the right-hand plot all known post-Newtonian parameters are treated as independent, while in the left-hand plot only three parameters $\psi_{0}, \psi_{2}$ and one of the remainingpost-Newtonian parameter are treated as independent and the procedure is repeated for each of the remaining parameters. The large SNR in LISA for SMBH binaries makes it possible to test various post-Newtonian effects, such as the tails of gravitational waves, tails of tails, the presence of log-terms, etc., associated with these parameters. Left-hand figure adapted from [46], right-hand figure reprinted with permission from [45]. (C) The American Physical Society. 
The range of mass ratios is also wide. LISA's central black holes might have masses between $10^{3}$ and $10^{7} M_{\odot}$. Inspiraling neutron stars and white dwarfs might have masses between 0.5 and $2 M_{\odot}$. Inspiraling stellar-population black holes might be in the range of $7-50 M_{\odot}$, while intermediatemass black holes formed by the first generation of stars (Population III stars) might have masses around $300 M_{\odot}$ or even $1000 M_{\odot}$. So the mass ratios might be anything in the range $10^{-7}$ to 1 .

The techniques that must be used to compute these signals depend on the mass ratio. Ratios near one are treated by post-Newtonian methods until the objects are so close that only numerical relativity can follow their subsequent evolution. For ratios below $10^{-4}$ (a dividing line that is rather very uncertain and that depends on the bandwidth being used to observe the system, i.e., on how long the approximation must be valid for), systems are treated by fully-relativistic perturbation theory, expanding in the mass ratio. Intermediate mass ratios have not been studied in much detail yet; they can probably be treated by post-Newtonian methods up to a certain point, but it is not yet clear whether their final stages can be computed accurately by either numerical relativity or perturbation theory.

Post-Newtonian methods have been extensively discussed above. The basics of perturbation theory underlying this problem are treated in two Living Reviews [292, 319]. Once one has sufficiently good waveform templates, there remain the challenge of finding weak signals in LISA's noise. This depends on a number of factors, including the rates of sources. A recent study by a number of specialists [170] has concluded that the event rate is high enough and the detection methods robust enough for us to be very optimistic that LISA will detect hundreds of these sources. In fact, the opposite problem might materialize: LISA might find it has a confusion problem for the detection of these sources, as for the galactic binaries. Recent estimates of the magnitude of the problem [66] suggest that LISA's noise may at worst be raised effectively by a factor of two, but in return one gets a large number of sources of this kind.

\subsubsection{Quantum gravity}

It seems inevitable that general relativity's description of nature will one day yield to a quantumbased description, involving uncertainties in geometry and probabilities in the outcome of gravitational observations. This is one of the most active areas of research in fundamental physics today, and there are many speculations about how quantum effects might show up in gravitational wave observations.

The simplest idea might be to try to find evidence for "gravitons" directly in gravitational waves, by analogy with the way that astronomers count individual photons from astronomical sources. But this seems doomed to failure. The waves that we can observe have very low frequency, so the energy of each graviton is extremely small. And the total energy flux of the waves is, as we have seen, enormous. So the number of gravitons in a detectable gravitational wave is far more than the number of photons in the light from a distant quasar.

Quantum gravity might involve new gravity-like fields, whose effects might be seen indirectly in the inspiral signals of black holes or neutron stars, as we have noted above. String theory might lead to the production of cosmic strings, which might be observed through their gravitational wave emission [142]. If our universe is just a 4-dimensional subspace of a large-scale 10 or 11-dimensional space, then dynamics in the larger space might produce gravitational effects in our space, and in particular gravitational waves [304].

It might be possible to observe the quantum indeterminacy of geometry directly using gravitational wave detectors, if Hogan's principle of holographic indeterminacy is valid [193]. Hogan speculates that quantum geometry might be manifested by an uncertainty in the position of a beam splitter, and that this could be the explanation for an unexpectedly large amount of noise at low frequencies in the GEO600 detector. In this connection it is interesting to construct, from fundamental constants alone, a quantity with the dimensions of amplitude spectral noise density 
$\left(S_{h}\right)^{1 / 2}$. This has units of $\mathrm{s}^{1 / 2}$, so one can define the "Planck noise power" $S_{\mathrm{Pl}}=t_{\mathrm{Pl}}=\left(G \hbar / c^{5}\right)^{1 / 2}$. Then the amplitude noise is $S_{\mathrm{Pl}}^{1 / 2}=\left(G \hbar / c^{5}\right)^{1 / 4}=2.3 \times 10^{-22} \mathrm{~Hz}^{-1 / 2}$. This is comparable to or larger than the instrumental noise in current interferometric gravitational wave detectors, as shown in Figure 5. This in itself does not mean that Planckian noise will show up in gravitational wave detectors, but Hogan argues that the particular design of GEO600 might indeed make it subject to this noise more strongly than other large interferometers. 


\section{Astrophysics with Gravitational Waves}

Gravitational radiation plays an observable role in the dynamics of many known astronomical systems. In some, such as cataclysmic variables [157] and neutron-star-binary systems [355], the role of gravitational radiation has been understood for years. In others, such as young neutron stars [34] and low-mass X-ray binaries [77], the potential importance of gravitational radiation has been understood only recently. As further observations, particularly at X-ray wavelengths, become available, the usefulness of gravitational radiation as a tool for modelling astronomical systems should increase [385].

At this point in the progress of gravitational wave detection, the greatest emphasis in calculations of sources is on prediction: trying to anticipate what might be seen. Not only is this important in motivating the construction of detectors, but it also guides details of their design and, very importantly, the design of data analysis methods. Historically, many predictions of emission strengths and the capability of detectors to extract information from signals have relied on estimates using the quadrupole formula. This was justifiable because, given the uncertainties in our astrophysical understanding of potential sources, more accurate calculations would be unjustified in most cases.

But these rough estimates are now being replaced by more and more detailed source models where possible. This applies particularly in two cases. One is binary orbits, where the pointmass approximation is good over a large range of observable frequencies, so that fully relativistic calculations (using the post-Newtonian methods described above) are not only possible, but are necessary for the construction of sensitive search templates in the data analysis. The second exception is the numerical simulations of the merger of black holes and neutron stars, where the dynamics is so complex that none of our analytic approximations offers us reliable guidance. In fact, these two methods are currently being joined to produce uniform models of signal evolution over as long an observation time as the signal allows. From these models we not only improve detection algorithms, but we also learn much more about the kinds of information that detections will extract from the signals.

Once gravitational waves have been observed, there will of course be a welcome shift of emphasis to include interpretation. The emphasis will be on extracting observable parameters (waveforms, polarizations, source location, etc.) from noisy data or data where (in the case of LISA) there is source confusion. These issues need considerably more attention than they have so far received.

\subsection{Interacting compact binaries}

The first example of the use of gravitational radiation in modelling an observed astronomical system was the explanation by Faulkner [157] of how the activity of cataclysmic binary systems is regulated. Such systems, which include many novae, involve accretion by a white dwarf from a companion star. Unlike accretion onto neutron stars, where the accreted hydrogen is normally processed quickly into heavier elements, on a white dwarf the unprocessed material can build up until there is a nuclear chain reaction, which results in an outburst of visible radiation from the system.

Now, in a circular binary system that conserves total mass and angular momentum, a transfer of mass from a more massive to a less massive star will make the orbit shrink, while a transfer in the opposite direction makes the orbit grow. If accretion onto a white dwarf begins with the dwarf as the less massive star, then the stars will draw together, and the accretion will get stronger. This runaway process stops when the stars are of equal mass, and then accretion begins to drive them apart again. Astronomers observed that in this phase accretion in certain very close binaries continued at a more or less steady rate, instead of shutting off as the stars separated more and more. Faulkner pointed out that gravitational radiation from the orbital motion would carry 
away angular momentum and drive the stars together. The two effects together result in steady accretion at a rate that can be predicted from the quadrupole formula and simple Newtonian orbital dynamics, and which is in good accord with observations in a number of systems.

Binaries consisting of two white dwarfs in very tight orbits will be direct LISA sources: we won't have to infer their radiation indirectly, but will actually be able to detect it. Some of them will also be close enough to tidally interact with one another, leading in some cases to mass transfer. Others will be relatively clean systems in which the dominant effect will be gravitational radiation reaction.

Observations during the last decade have identified a number of such systems with enough confidence to predict that LISA should see their gravitational waves. These are called verification binaries: if LISA does not see them then either the instrument is not working properly or general relativity is wrong! For a review of verification binaries, see [349].

\subsubsection{Resolving the mass-inclination degeneracy}

Gravitational-wave-polarization measurements can be very helpful in resolving the degeneracy that occurs in the measurement of the mass and inclination of a binary system. As is well known, astronomical observations of binaries cannot yield the total mass but only the combination $m \sin \iota$, where $\iota$ is the inclination of the binary's orbit to the line of sight. However, measurement of polarization can determine the angle $\iota$ since the polarization state depends on the binary's inclination with the line of sight.

For instance, consider a circular binary system with total mass $M$ at a distance $D$. Suppose its orbital angular momentum vector makes an angle $\iota$ with the line of sight (the standard definition of the inclination of a binary orbit). The two observed polarizations are given in the quadrupole approximation by Equation (122). We can eliminate the distance $R$ between the stars that is implicit in the velocity $v=R \omega$ (where $\omega$ is the instantaneous angular velocity of the orbit, the derivative of the orbital phase function $\varphi(t)$ ) by using the Newtonian orbital dynamics equation $\omega^{2}=M / R^{3}$. Then we find

$$
h_{+}=\frac{2 \nu M}{D}[\pi M f(t)]^{2 / 3}\left(1+\cos ^{2} \iota\right) \cos [2 \varphi(t)], \quad h_{\times}=\frac{4 \nu M}{D}[\pi M f(t)]^{2 / 3} \cos \iota \sin [2 \varphi(t)],
$$

where $M$ is the total mass of the binary and, as before, $\nu$ is the symmetric mass ratio $m_{1} m_{2} / M^{2}$. The frequency $f=\omega / \pi$ is the gravitational wave frequency, twice the orbital frequency. Notice that, consistent with Equation (30), the masses of the stars enter these equations only in the combination $\mathcal{M}=\nu^{3 / 5} M$.

It is clear that the ratio of the two polarization amplitudes determines the angle $\iota$. In this connection it is interesting to relate the polarization to the orientation. When the binary is viewed from a point in its orbital plane, so that $\iota=\pi / 2$, then $h_{\times}=0$; the radiation has pure + polarization. From the observer's point of view, the motion of the binary stars projected onto the sky is purely linear; the two stars simply go back and forth across the line of sight. This linear projected motion results in linearly polarized waves. At the other extreme, consider viewing the system down its orbital rotation axis, where $\iota=0$. The stars execute a circular motion in the sky, and the polarization components $h_{+}$and $h_{\times}$have equal amplitude and are out of phase by $\pi / 2$. This is circularly polarized gravitational radiation. So, when the radiation is produced in the quadrupole approximation, the polarization has a very direct relationship to the motions of the masses when projected on the observer's sky plane. If the radiation is produced by higher multipoles it becomes more complex to make these relations, but it can be done. For example, see [333] for the case of current quadrupole radiation, which is emitted by the r-mode instability discussed in Section 7.3.4.2 below.

While a single detector is linearly polarized, it can still measure the two polarizations if the signal has a long enough duration for the detector to turn (due to the motion of the Earth) and

Living Reviews in Relativity

http://www. livingreviews.org/lrr-2009-2 
change the polarization component it measures. Alternatively, a network of three detectors can determine the polarization and location of the source even over short observation times.

Such a measurement would lead to a potentially very interesting interplay between gravitational and electromagnetic observations, with applications in the observations of isolated neutrons stars, binary systems, etc. And would lead to synergies, for example, between the LISA and Gaia [289] missions.

\subsection{Black hole astrophysics}

Black holes are the most relativistic systems possible. Observing gravitational waves from them, individually or in binaries, helps to test some of the predictions of general relativity in the strongly nonlinear regime, such as the tails of gravitational waves, spin-orbit coupling induced precession, nonlinear amplitude terms, hereditary effects, etc [360, 84, 83, 322]. They are also good test beds to constrain other theories of gravity. Gravitational waves - emitted either during the inspiral and merger of rotating SMBHs or when a stellar-mass compact object falls into a SMBH - can be used to map the structure of spacetime and test uniqueness theorems on rotating black holes [360]. LISA will be able to see the formation of massive black holes at cosmological distances by detecting the waves emitted in the process [360]. We give below a brief discussion of the physics that will follow from the observation of gravitational waves from black holes.

\subsubsection{Gravitational waves from stellar-mass black holes}

Astronomers now recognize that there is an abundance of black holes in the universe. Observations across the electromagnetic spectrum have located black holes in X-ray binary systems in our galaxy in the centers of star clusters, and in the centers of galaxies.

These three classes of black holes have very different masses. Stellar black holes typically have masses of around $10 M_{\odot}$, and are thought to have been formed by the gravitational collapse of the center of a large, evolved red giant star, perhaps in a supernova explosion. Black holes in clusters have been found in the range of $10^{4} M_{\odot}$, and are called intermediate-mass black holes. Black holes in galactic centers have masses between $10^{6}$ and $10^{10} M_{\odot}$, and are called SMBHs. The higher masses are found in the centers of active galaxies and quasars. The history and method of formation of intermediate-mass and supermassive black holes are not yet well understood.

All three kinds of black hole can radiate gravitational waves. According to Figure 2, stellar black-hole radiation will be in the ground-based frequency range, while galactic holes are detectable only from space. Intermediate-mass black holes may lie at the upper end of the LISA band or between LISA and ground-based detectors. The radiation from an excited black hole itself is strongly damped, lasting only a few cycles at its natural frequency [see Equation (12) with $R=2 M]$ :

$$
f_{B H} \sim 1000\left(\frac{M}{10 M_{\odot}}\right)^{-1} \mathrm{~Hz}
$$

\subsubsection{Stellar-mass black-hole binaries}

Radiation from stellar-mass black holes is expected mainly from coalescing binary systems, when one or both of the components is a black hole. Although black holes are formed more rarely than neutron stars, the spatial abundance of binary systems consisting of neutron stars with black holes, or of two black holes, is amplified relative to neutron-star binaries because binary systems are much more easily broken up when a neutron star forms than when a black hole forms. When a neutron star forms, most of the progenitor star's mass $\left(6 M_{\odot}\right.$ or more $)$ must be expelled from the system rapidly. This typically unbinds the binary: the companion star has the same speed as before but 
is held to the neutron star by only a fraction of the original gravitational attraction. Observed neutron-star binaries are thought to have survived because the neutron star was coincidentally given a kick against its orbital velocity when it formed. When a black hole forms, most of the original mass may simply go down into the hole, and the binary will have a higher survival probability. However, this argument may not lead to observable black hole binaries; there is a possibility that systems that would form black holes close enough to coalesce in a Hubble time do not become binaries, but rather the two progenitor stars are so close that they merge before forming black holes.

On the other hand, double black-hole binaries may in fact be formed abundantly by capture processes in globular clusters, which appear to be efficient factories for black-hole binaries [295]. Being more massive than the average star in a globular cluster, black holes sink towards the center, where three-body interactions can lead to the formation of binaries. The key point is that these binaries are not strongly bound to the cluster, so they can easily be expelled by later encounters. From that point on they evolve in isolation, and typically have a lifetime shorter than $10^{10} \mathrm{yrs}$.

The larger mass of stellar black-hole systems makes them visible from a greater distance than neutron-star binaries. If the abundance of binaries with black holes is comparable to that of neutron-star binaries, black hole events will be detected much more frequently than those involving neutron stars. They may even be seen by first-generation detectors in the S5 science run of the LSC (see Section 4.3.1), although that is still not very probable, even with optimistic estimates of the black-hole binary population. It seems very possible, however, that the first observations of binaries by interferometers will eventually be of black holes.

More speculatively, black hole binaries may even be part of the dark matter of the universe. Observations of Massive Compact Halo Objects (MACHOs) - microlensing of distant stars by compact objects in the halo of our galaxy - have indicated that up to half of the galactic halo could be made up of dark compact objects of $0.5 M_{\odot}$ [25, 351]. This is difficult to understand in terms of stellar evolution, as we understand it today: neutron stars and black holes should be more massive than this, and white dwarfs of this mass should be bright enough to have been identified as the lensing objects. One speculative possibility is that the objects were formed primordially, when conditions may have allowed black holes of this mass to form. If so, there should also be a population of binaries among them, and occasional coalescences should, therefore, be expected. In fact, the abundance would be so high that the coalescence rate might be as large as one every 20 years in each galaxy, which is higher than the supernova rate. Since binaries are maximally non-axisymmetric, these systems could be easily detected by first-generation interferometers out to the distance of the Virgo Cluster [265].

The estimates used here of detectability of black hole systems depend mainly on the radiation emitted as the orbit decays, during which the point-particle post-Newtonian approximation should be adequate. But the inspiral phase will, of course, be followed by a burst of gravitational radiation from the merger of the black holes that will depend in detail on the masses and spins of the objects. Numerical simulations of such events will be used to interpret this signal and to provide templates for the detection of black holes too massive for their inspiral signals to be seen. There is an abundance of information in these signals: population studies of the masses and spins of black holes, studies of typical kick velocities for realistic mergers, tests of general relativity.

\subsubsection{Intermediate-mass black holes}

Intermediate-mass black holes, with masses between $100 M_{\odot}$ and $10^{4} M_{\odot}$, are expected on general evolutionary grounds, but have proved hard to identify because of their weaker effect on surrounding stellar motions. Very recently [275] strong evidence has been found for such a black hole in the star cluster Omega Centauri. If such black holes are reasonably abundant, then they may be LISA sources when they capture a stellar-mass black hole or a neutron star from the surrounding cluster.

Living Reviews in Relativity

http: //www . livingreviews .org//rr-2009-2 
For these merger events the mass ratio is not as extreme as for EMRIs, and so these are accordingly called IMRIs: Intermediate Mass-Ratio Inspirals.

The problem of modelling the signals from these systems has not yet been fully studied. If these signals can be detected, they will tell us how important black holes were in the early stellar population, and whether these black holes have anything to do with the central black holes in the same galaxies.

\subsubsection{Supermassive black holes}

Gravitational radiation is expected from SMBHs in two ways. In one scenario, two massive black holes spiral together in a much more powerful version of the coalescence we have just discussed. The frequency is much lower, in inverse proportion to their masses, and the amplitude is higher. Equation (128) implies that the effective signal amplitude (which is what appears in the expression for the SNR) is almost linear in the masses of the black holes, so that a signal from two $10^{6} M_{\odot}$ black holes will have an amplitude $10^{5}$ times bigger than the signal from two $10 M_{\odot}$ holes at the same distance. Even allowing for differences in technology, this indicates why space-based detectors will be able to study such events with a very high SNR, no matter where in the universe they occur. Observations of coalescing massive black-hole binaries will therefore provide unique insight into the behavior of strong gravitational fields in general relativity.

The event rate for such coalescences is not easy to predict, but is likely to be large. It seems that the central core of most galaxies may contain a black hole of at least $10^{6} M_{\odot}$. This is known to be true for our galaxy [151] and for a very large proportion of other galaxies that are near enough to be studied in sufficient detail [312]. SMBHs (up to a few times $10^{9} M_{\odot}$ ) are believed to power quasars and active galaxies, and there is a good correlation between the mass of the central black hole and the velocity dispersion of stars in the core of the host galaxy [174].

If black holes are formed with their galaxies, in a single spherical gravitational collapse event, and if nothing happens to them after that, then coalescences will never be seen. But this is unlikely for two reasons. First, it is believed that galaxies may have formed through the merger of smaller units, sub-galaxies of masses upwards of $10^{6} M_{\odot}$. If these units had their own black holes, then the mergers would have resulted in the coalescences of many of the black holes on a timescale shorter than the present age of the universe. This would give an event rate of several mergers per year in the universe, most of which would be observable by LISA, if the more massive black hole is not larger than about $10^{7} M_{\odot}$. If the $10^{6} M_{\odot}$ black holes were formed from smaller black holes in a hierarchical merger scenario, then the event rate could be hundreds or thousands per year. The second reason is that we see large galaxies merging frequently. Interacting galaxies are common, and if galaxies come together in such a way that their central black holes both remain in the central core, then dynamical friction with other stars will bring them close enough together to allow gravitational radiation to bring about a merger on a timescale of less than $10^{10} \mathrm{yrs}$. There is considerable evidence for black hole binaries in a number of external galaxies [255]. There is even a recent report of an SMBH having been ejected from a galaxy, possibly by the kick following a merger [222] and of an SMBH binary that will coalesce in about 10,000 yrs [373]!

Besides mergers of holes with comparable masses, the capture of a small compact object by a massive black hole can also result in observable radiation. The tidal disruption of main-sequence or giant stars that stray too close to the black hole is thought to provide the gas that powers the quasar phenomenon. These disruptions are not expected to produce observable radiation. But the clusters will also contain a good number of neutron stars and stellar-mass black holes. They are too compact to be disrupted by the black hole, even if they fall directly into it.

Such captures, therefore, emit a gravitational wave signal that will be well approximated as that from a point mass near the black hole. This will again be a chirp of radiation, but in this case the orbit may be highly eccentric. The details of the waveform encode information about the 
geometry of spacetime near the black hole. In particular, it may be possible to measure the mass and spin of the black hole and thereby to test the uniqueness theorem for black holes. The event rate is not very dependent on the details of galaxy formation, and is probably high enough for many detections per year from a space-based detector [32], provided that theoretical calculations give data analysts accurate predictions of the motion of these point particles over many hundreds of thousands of orbits. These Extreme Mass-Ratio Inspiral sources (EMRIs) are a primary goal of the LISA detector. By observing them, LISA will provide information about the stellar population near central black holes. When combined with modelling and spectroscopic observations, this will facilitate a deep view of the centers of galaxies and their evolution.

\subsection{Neutron star astrophysics}

\subsubsection{Gravitational collapse and the formation of neutron stars}

The event that forms most neutron stars is the gravitational collapse that results in a supernova. It is difficult to predict the waveform or amplitude expected from this event. Although detecting this radiation has been a goal of detector development for decades, little more is known about what to expect than 30 years ago. The burst might be at any frequency between $100 \mathrm{~Hz}$ and $1 \mathrm{kHz}$, and it might be a regular chirp (from a rotating deformed core) or a more chaotic signal (from convective motions in the core). Considerable energy is released by a collapse, and on simple energetic grounds this source could produce strong radiation: if the emitted energy is more than about $0.01 M_{\odot}$, then second-generation detectors would have no trouble seeing events that occur in the Virgo Cluster. This energetic consideration drove the early development of bar detectors.

But numerical simulations tell a different story, and it seems very likely that radiation amplitudes will be much smaller, as described in Section 3. Such signals might be detectable by second-generation detectors from a supernova in our galaxy, but not from much greater distances. When they are finally detected, the gravitational waves will be extremely interesting, providing our only information about the dynamics inside the collapse, and helping to determine the equation of state of hot nuclear matter.

If gravitational collapse forms a neutron star spinning very rapidly, then it may be followed by a relatively long period (perhaps a year) of emission of nearly monochromatic gravitational radiation, as the r-mode instability (Section 7.3.4) forces the star to spin down to speeds of about $100-200 \mathrm{~Hz}$ [278]. If as few as $10 \%$ of all the neutron stars formed since star formation began (at a redshift of perhaps four) went through such a spindown, then they may have produced a detectable random background of gravitational radiation at frequencies down to $20 \mathrm{~Hz}$ [324].

\subsubsection{Neutron-star-binary mergers}

When two neutron stars merge, they will almost certainly have too much mass to remain as a star, and will eventually collapse to a black hole, unless they can somehow expel a significant amount of mass. The collision heats up the nuclear matter to a point where, at least initially, thermal pressure becomes significant. Numerical simulations can use theoretical equations of state (such as that of Lattimer and Swesty [233]) to predict the merger radiation, and observations will then test the nuclear physics assumptions that go into the equation of state. Simulations show that the choice of equation of state makes a big difference to the emitted waveform, as do the masses of the stars: there is no mass scaling as there is for black holes [60].

When a neutron star encounters a black hole in a stellar compact binary merger, the star may not be heated very much by the tidal forces, and the dynamics may be governed by the cold nuclearmatter equation of state, about which there is great uncertainty. Again, comparing observed with predicted waveforms may provide some insight into this equation of state. Simulations suggest that these systems may give rise to many of the observed short, hard gamma-ray bursts [155, 336].

Living Reviews in Relativity

http://www.livingreviews.org/lrr-2009-2 
Simultaneous gravitational wave and gamma ray detections would settle the issue and open the way to more detailed modeling of these systems.

\subsubsection{Neutron-star normal mode oscillations}

Gravitational wave observations at high frequencies of neutron-star vibrations may also constrain the cold-matter equation of state. In Figure 2 there is a dot for the typical neutron star. The corresponding frequency is the fundamental vibrational frequency of such an object. In fact, neutron stars have a rich spectrum of nonradial normal modes, which fall into several families: f, g, p, w, and r-modes have all been studied. These have been reviewed by Andersson and Comer [35]. If their gravitational wave emissions can be detected, then the details of their spectra would be a sensitive probe of their structure and of the equation of state of neutron stars, in much the same way that helioseismology probes the interior of the sun. Even knowing accurately the frequency and decay time of just the fundamental $\ell=2 \mathrm{f}$-mode would be enough to eliminate most current equations of state [36].

This is a challenge to ground-based interferometers, which have so far focussed their efforts on frequencies below $1 \mathrm{kHz}$. But Advanced LIGO and the upgraded GEO-HF detector (Section 4.3.1) may have the capability to perform narrow-banding and enhance their sensitivity considerably at frequencies up to perhaps $2 \mathrm{kHz}$, which could put the f-modes of neutron stars into range.

The f-modes of neutron stars, which could be excited by glitches or by the nuclear explosions on accreting neutron stars that are thought to produce X-ray flares and soft gamma-ray repeater events. The rise-time of X-ray emission can be as short as a few milliseconds [173], which might be impulsive enough to excite acoustic vibrations. If the rise time of the explosion matches the period of the mode well enough, then a substantial fraction of the energy released could go into mechanical vibration, and almost all of this fraction would be carried away by gravitational waves, since other mode-damping mechanisms inside neutron stars are much less efficient.

Radio-pulsar glitches seem to release energies of order $10^{35} \mathrm{~J}$, and X-ray and gamma ray events can be much more energetic. Using Equation (20), we can estimate that the release of that much energy into gravitational waves at $2 \mathrm{kHz}$ at a distance of $1 \mathrm{kpc}$ would create a wave of effective amplitude around $3 \times 10^{-22}$. (The effective amplitude assumes we can do matched filtering, which in this case is not very difficult.) This kind of amplitude should be within the reach of Advanced LIGO (Figure 5) and perhaps GEO-HF, provided they implement narrowbanding. This will not be easy, either scientifically or operationally, but the payoff in terms of our understanding of neutron star physics could be very substantial.

Observations of these modes would immediately constrain the cold-matter nuclear equation of state in significant ways [36, 35].

In fact, modes of neutron stars may have already been observed in X-rays [386]. But these are likely to be crustal modes, whose restoring force is the shear strength of the crust. While the physics of the crust is interesting in itself, such observations provide only weak constraints on the interior physics of the neutron star.

\subsubsection{Stellar instabilities}

7.3.4.1 The CFS instability. In 1971 Chandrasekhar [111] applied the quadrupole formula to calculate the corrections to the eignefrequencies of the normal mode vibrations of rotating stars, and he found to his surprise that some modes were made unstable, i.e., that coupling to gravitational radiation could destabilize a rotating star. Subsequent work by Friedman and Schutz [166] showed that there was a key signature for the mode of a Newtonian star that would be unstable in general relativity. This was the pattern speed of the mode, i.e., the angular velocity at which the crests of the pattern rotated about the rotation axis of the star. If this speed was in the same sense as the rotation of the star, but slower than the star, then the mode would be unstable in a perfect-fluid 
star. This instability has come to be known as the CFS instability, after the three authors who explained it.

The basic theory was developed for perfect-fluid stars. However, Lindblom and Detweiler [238] showed that the effect of viscosity ran counter to that of radiation reaction, so that the instability was strongest in modes with the longest wavelengths, i.e., in the quadrupolar modes. Full numerical calculations on Newtonian stellar models with realistic viscosity models showed [239] that the standard fundamental and acoustic modes of rotating neutron stars were not vulnerable to this instability. Subsequent work on fully relativistic models [347] has hinted that the instability may be stronger than the Newtonian models indicate, but it is still at the margins of astrophysical interest.

7.3.4.2 The r-mode instability. The situation changed in 1997 when Andersson [34] pointed out that there is another class of modes of Newtonian stars that should be unstable in the same way, but which had not been studied in this context before, the Rossby or r-modes. These are momentum-dominated modes, where the gravitational radiation comes from the currentquadrupole terms, rather than from the mass quadrupole. Investigations by a number of authors $[241,37,278]$ have shown that this instability could be very strong in hot, rapidly-rotating stars. This is particularly relevant to young neutron stars, which may well be formed with rapid spin and which will certainly be hot. For their first year, stars spinning faster than about $100 \mathrm{~Hz}$ could spin down to about $100 \mathrm{~Hz}$ by losing angular momentum to gravitational radiation. The instability might also operate in old accreting neutron stars, such as those in LMXB X-ray binaries (see the next section).

However, the instability is, like other CFS instabilities, sensitive to viscosity, and there is great uncertainty about the amount of viscosity inside neutron stars [240, 228, 35].

\subsubsection{Low-mass X-ray binaries}

Observations by the Rossi satellite (RXTE) have given evidence that the class of X-ray sources called Low-Mass X-ray Binaries (LMXB's) contains neutron stars with a remarkably narrow range of spins, between perhaps $250 \mathrm{~Hz}$ and $320 \mathrm{~Hz}$ [376]. These are systems in which it is believed that neutron stars are spun up from the low angular velocities they have after their lifetime as normal pulsars to the high spins that millisecond pulsars have. One would expect, therefore, that the spins of neutron stars in such systems would be spread over a wide range. The fact that they are not requires an explanation.

The most viable explanation offered so far is the suggestion of Bildsten [77] that gravitational radiation limits the rotation rate. The proposed mechanism is that anisotropic accretion onto the star creates a temperature gradient in the crust of the neutron star, which in turn creates a gradient in the mass of the nucleus that is in local equilibrium, and this in turn creates a density gradient that leads, via the rotation of the star, to the emission of gravitational radiation. This radiation carries away angular momentum, balancing that which is accreted, so that the star remains at an approximately constant speed.

According to the model, the gravitational wave luminosity of the star is proportional to the measured flux of X-rays, since the X-ray flux is itself proportional to the accreted angular momentum that has to be carried away by the gravitational waves. If this model is correct, then the X-ray source Sco X-1 might be marginally detectable by advanced interferometers, and other similar systems could also be candidates [385].

\subsubsection{Galactic population of neutron stars}

Neutron stars are known to astronomy through the pulsar phenomenon. As radio surveys improve, the number of known pulsars is pushing up toward 2000. There is a public catalogue on the

Living Reviews in Relativity

http://www. livingreviews.org/lrr-2009-2 
web [55]. But the galactic population of neutron stars is orders of magnitude larger, perhaps as many as $10^{8}$. Most are much older than typical pulsars, which seem to stop emitting after a few million years. X-ray surveys reveal a number of unidentified point sources, which might be hot neutron stars, but older neutron stars are probably not even hot enough to show up in such surveys.

Gravitational wave observations have the potential to discover more neutron stars, but in the foreseeable future the numbers will not be large. Spinning neutron stars can be found in searches for continuous-wave signals, but there is no a priori reason to expect significant deformations that would lead to large gravitational wave amplitudes. One mechanism, proposed by Cutler [127], is that a large buried toroidal magnetic field could, by pulling in the waist of a spinning star, turn it into a prolate spheroid. This is classically unstable and would tip over and spin about a short axis, emitting gravitational waves. Millisecond pulsars could, in principle, be spinning down through the emission of gravitational waves in this way. Only deep observations by Advanced LIGO could begin to probe this possibility.

In fact, strong emission of gravitational waves is in some sense counterproductive, since it causes a neutron star to spin down and move out of the observing band quickly. This places important limits on the likely distribution of observable continuous-wave amplitudes from neutron stars [219]. This is important input into the blind searches for such signals being conducted by the LSC.

Radio observations of pulsars have, of course, revealed a fascinating population of binary systems containing neutron stars, including the original Hulse-Taylor pulsar [201] and the double pulsar PSR J0737-3039 [246]. But radio surveys only cover a small fraction of our galaxy, so there may be many more interesting and exotic systems waiting to be discovered, including neutron stars orbiting black holes. In fact, not all neutron stars are pulsars, so there are likely to be nearby binary systems containing neutron stars that are not known as pulsars at all.

LISA has enough sensitivity to detect all such binaries in the galaxy whose gravitational wave emission is above $1 \mathrm{mHz}$, i.e., with orbital periods shorter than half an hour. Below that frequency, systems may just blend into the confusion noise of the white-dwarf background, unless they are particularly close. The Hulse-Taylor system is a bit below the LISA band, and even its higher harmonics are likely to be masked by the dense confusion noise of white-dwarf galaxies at low frequencies. Double pulsars should be detectable by LISA with low SNR (around five in five years) above the confusion background at a frequency of $0.2 \mathrm{mHz}$ [210]. In all, LISA might detect several tens or even hundreds of double neutron-star systems, and potentially even a handful of double black hole binaries.

Neutron stars are the fossils of massive stars, and so a population census of binaries can help normalize our galaxy's star-formation rate in the past. The mass distribution of such systems will also be of interest: do all neutron-star binaries have stars whose masses are near $1.4 M_{\odot}$, or is this only true of systems that become pulsars? LISA observations are likely to illuminate many puzzles of stellar evolution.

Finally, it is possible to search for gravitational waves from individual spinning neutron stars in binary systems. Although more rare than isolated neutron stars, these systems might have a different history and a different distribution of amplitudes. Searches are planned by the LSC, but they are difficult to do, since the parameter space is even larger than for isolated pulsars.

\subsection{Multimessenger gravitational-wave astronomy}

Multimessenger gravitational-wave astronomy refers to coordinated observations using different kinds of radiation and information carriers: electromagnetic, neutrino, cosmic ray, and gravitational wave. Joint coordinated observing has much to offer gravitational wave detection, by allowing it to target known interesting sources or locations, thereby reducing the parameter space that must be searched and improving the confidence of a detection. Even more importantly, the information obtained from gravitational wave observations is typically complementary to that which one can get 
from electromagnetic astronomy, and so there are big science gains to be realized from coordinated observations.

One can distinguish three broad classes of coordinated observations: triggered gravitationalwave searches, follow-up electromagnetic observations, and parameter refinement.

- Triggered searches use transient electromagnetic events, such as gamma-ray bursts, to narrow down the window of time for a search in the gravitational-wave data stream, and possibly also to restrict the ranges of various parameters. Since gravitational-wave-signal data is recorded, it is no problem to go back to data at the time of the triggering event and search it. This helps to lower the detection threshold, since gravitational wave events need, in this case, to be significant over a time scale of a few minutes rather than, say, an entire year.

- Follow-up searches use a, possibly tentative, gravitational-wave detection to mark an area in the sky and a timeframe for an electromagnetic search. A very interesting example of this will occur with LISA, which will be able to predict the location and time of the coalescence of two SMBHs with reasonable accuracy at least a week in advance. This will allow sufficient time for telescopes in all the electromagnetic wavelengths to prepare to observe the event.

- Parameter refinement refers to the use of electromagnetic obeservations of potential gravitational wave sources to improve the values of the parameters that must be used in the gravitational wave search. This has already been used in LSC searches, for example in trying to detect radiation from known radio pulsars: radio observations during the gravitationalwave observation period were used to track the changing frequency of the pulsar [11].

Finding electromagnetic counterparts to gravitational wave observations is important, of course, for learning about the nature of the events. But it has a more subtle benefit: it generally improves significantly the accuracy with which parameters can be estimated from the gravitational wave observation. The reason is that one of the biggest sources of parameter uncertainty is the sky location of a gravitational wave source. Interferometers have broad antenna patterns, which is helpful in that they can monitor essentially the entire sky continuously, but which means that directional information for transient events can come only from time delay information among different detectors. The simple Rayleigh limit $\lambda_{g w} / D$ for ground-based interferometers gives angular accuracies on the order of several degrees, divided by the amplitude SNR (never smaller than 5 for any reasonable detection). The covariance of angular errors with uncertainties in other parameters (distance, polarization, stellar masses, etc) is usually significant. Therefore, if a follow-up electromagnetic observation can provide a more accurate position, this can also improve the determination of all the other parameters measured gravitationally.

Triggered searches are already being performed by the LSC for gravitational waves associated with gamma-ray bursts [14]. The nondetection of any gravitational waves associated with the gamma-ray burst GRB 070201 showed that it was not created by the merger of neutron stars in the nearby galaxy M31, despite its positional coincidence on the sky [16]. In addition, the gravitational wave detectors are monitoring the triggers provided by both high-energy and lowenergy neutrino detectors in order to get instant warning of a supernova in our galaxy or of some more exotic event further away. As we have noted above, X-ray flares from neutron stars may signal normal-mode radiation from acoustic vibrations.

Triggers may also allow the first detection of gravitational waves from the normal modes of neutron stars, which as mentioned in Section 7.3.3, would provide our first "view" inside these exotic objects. These triggers could be radio-pulsar glitches, X-ray flares, or even the formation and subsequent ringdown of a neutron star.

Follow-up observations of neutron-star-binary coalescence events are likely to be particularly informative. It is possible that these events are associated with short gamma-ray bursts, in which

Living Reviews in Relativity

http: //www. livingreviews.org/lrr-2009-2 
case most events are missed because of the narrow beaming of the gamma rays. Gravitational waves, by contrast, are emitted nearly isotropically, so that they will pick out essentially all such events within the range of the detectors, and astronomers can subsequently search for afterglows and prompt X- and gamma-ray emission. The ability to study such events from all aspect angles will help model them reliably. Even if coalescences are not associated with gamma-ray bursts, it is difficult to imagine that they will not produce visible afterglows or other transient electromagnetic events that would presumably not have been recognized before. The same considerations apply to coalescences of neutron stars and black holes.

Gravitational wave events may also provide our first notice of a gravitational collapse event, if the event is a strong radiator and is too far away for neutrino detectors to see it. While supernova simulations generally suggest that the amplitude of emitted gravitational waves is small [147], numerical simulations of the aftermath of neutron-star coalescence suggest the possibility of very powerful gravitational-wave emission [60]. While this event seems to lead inevitably to a black hole, because the total mass is too large for a single neutron star, neutron stars might occasionally be formed in this way by mergers of white dwarfs, again with strong rotation and the possibility of the emission of strong gravitational radiation. In this connection the suggestion of Arons [43] that at least some magnetars are formed in events of some kind that involve strong magnetic field braking but also strong gravitational wave emission, and that these events are the source of the ultra-high-energy cosmic rays whose source, is so far unexplained [384].

LISA offers particularly interesting opportunities for follow-up observations with electromagnetic waves, beyond the direct monitoring of the merger events for SMBHs mentioned above. Because SMBHs often carry accretion disks, the merger event may be followed by the turning on of accretion after a delay of, perhaps, a year or so [257]. The merger may also cause a prompt shock in surrounding gas, due to the essentially instantaneous loss of several percent of the gravity of the central mass. These or similar effects may make it possible to identify the galaxies that host LISA mergers, which in turn will allow one to associate a redshift with the luminosity distance that the gravitational wave event provides. This will be important for LISA's cosmographic capabilities (next section).

LISA will look for close white-dwarf binaries in our galaxy and will probably see thousands of them. White-dwarf binaries never reach the last stable orbit, which would occur at roughly $1.5 \mathrm{kHz}$ for these masses. Instead they undergo a tidal interaction and can either disrupt at much lower frequency or end up as AM CVns (see, for instance, [341, 271]). In the latter case, we have a close white-dwarf binary with orbital periods of minutes or hours, wherein the smaller of the two stars transfers mass to the more massive one. This mass loss leads to an increase in the orbital period as a result of redistribution of the angular momentum. So far only a handful of AM CVn systems are known. LISA could potential discover a lot more of these as their orbital periods are right in the heart of LISA's sensitivity band and simultaneous observation of these systems in the gravitational and electromagnetic window has huge impacts on the science we can learn about these end products of stellar evolution and their eventual fate.

For each resolved white-dwarf binary LISA can determine the orbital period and the spatial orientation of the orbit, and it can give a relatively crude position. If the orbit is seen to decay during the observation, LISA can determine the distance to the binary. If the binary is known from optical or X-ray observations, then this can be very valuable additional information about the system, again complementary to that which is normally available from the electromagnetic observation. Even for systems that have not been identified, LISA's census of white-dwarf binaries will provide important statistics (on the mass function, distribution of separations, etc) that should lead to a better understanding of white-dwarf and binary evolution.

In the near term, one of the most practical applications of multimessenger astronomy is to use electromagnetic observations to refine the values of key search parameters for the gravitational wave data analysis. This has been extensively discussed for possible observations of low-mass 
X-ray binaries, as described in Section 7.3.5. Watts et al. [385] surveyed the known ranges of parameters, such as spin rates and orbital parameters, and concluded that they need to be narrowed considerably if a practical search were to be possible, not just because of the computer power required, but more importantly because of the loss of significance if too large a parameter space has to be searched. 


\section{Cosmology with Gravitational Wave Observations}

Gravitational wave observations may inform us about cosmology in at least two ways: by studies of individual sources at cosmological distances that give information about cosmography (the structure and kinematics of the universe) and about early structure formation, and by direct observation of a stochastic background of gravitational waves of cosmological origin. In turn, a stochastic background could either be astrophysical in origin (generated by any of a myriad of astrophysical systems that have arisen since cosmological structure formation began, as described in Section 8.2.2), or it could come from the Big Bang itself (generated by quantum processes associated with inflation or with spontaneous symmetry breaking in the extremely early universe, as described in Section 8.2.1). The observation of a cosmic gravitational wave background (CGWB) is probably the most fundamentally important observation that gravitational wave detectors can make. But the astrophysical gravitational wave background (AGWB) also contains important information and may mask the CGWB over much of the accessible spectrum.

The detection of discrete sources at cosmological distances will require high sensitivity. Advanced ground-based detectors should be able to see a few individual sources (mainly stellar-mass black hole binaries) at redshifts approaching 1 , with which they may be able to make a good determination of the Hubble constant. But LISA's observations of the coalescences of massive black hole binaries at all redshifts should make LISA a significant tool for cosmography. We examine cosmography measurements in Section 8.3. These high- $z$ observations may also contain interesting information about early structure formation, such as the relationship between SMBH formation and galaxy formation. We have mentioned this already in Section 7.2.4.

Both kinds of detectors will search for a stochastic background in their own wave band. As we have seen earlier, LISA will almost certainly detect an AGWB from binary systems in our galaxy, and both LISA and advanced ground-based detectors may see a CGWB, if the more optimistic estimates of its strength are correct. But scientists are already sketching designs for a mission to follow LISA with much higher sensitivity, dedicated to observing the CGWB from inflation. Stochastic searches are described in Section 8.1.2.

Other detection methods are also being used to probe the spectrum of the background radiation at longer wavelengths. Pulsar timing observations (Section 8.1.3) are already being used to set limits on the background at periods of a few years, and they will reach much greater sensitivity when coherent antenna arrays (like the Square Kilometer Array [224, 107]) are available. And observations of the temperature fluctuations of the cosmic microwave background (Section 8.1.4) have the potential to reveal the gravitational wave content of the universe at the redshift of decoupling, which means at wavelength scales comparable to the size of the universe [300, 214].

Before examining the details of detection, we begin by examining the statistics of a random gravitational wave background. A good introduction to the theory of the CGWB is the set of lectures by Bruce Allen at the 1996 Les Houches summer school [27]. The first paper of the LSC on searches for a stochastic background [1] also contains a brief introduction.

\subsection{Detecting a stochastic gravitational wave background}

\subsubsection{Describing a random gravitational wave field}

By definition, a stochastic background of gravitational waves is a superposition of waves arriving at random times and from random directions, overlapping so much that individual waves are not identifiable. We assume that there are so many sources (either astrophysical sources or the quantum fluctuations that create the CGWB) that individual ones are not distinguishable. Such a gravitational wave field will appear in detectors as a time-series noise, which by the central limit theorem should have a Gaussian-normal distribution function if there are enough overlapping sources. This kind of background will compete with instrumental noise. It will be detectable by 
a single detector, if it is stronger than instrumental noise, but a weaker background could still be detected by using a pair of detectors and looking for a correlated component of their "noise" output, on the assumption that their instrumental noise is not correlated.

As a random phenomenon, the gravitational wave fields at two different locations are uncorrelated, because gravitational waves arrive from all directions and at all frequencies. It might, therefore, be thought that two detectors' responses would be correlated only if they were located at the same position. But if one considers one component of the wave field with a single frequency, then it is clear that there will be strong correlations between points if they are separated along the wave's propagation direction by much less than a wavelength. We shall see that these frequencydependent correlations allow one to detect a background by cross-correlating the output of two separated detectors, albeit with less sensitivity than if they were co-located. We shall consider cross-correlation as a detection method in Section 8.1.2.

Random gravitational waves are conventionally described in terms of their energy density spectrum $\rho_{\mathrm{gw}}(f)$, rather than their mean amplitude. It is convenient to normalize this energy density to the critical density $\rho_{c}$ required to close the universe, which is given in terms of the Hubble constant $H_{0}$ as

$$
\rho_{c}=3 H_{0}^{2} / 8 \pi G
$$

We then define

$$
\Omega_{\mathrm{gw}}:=\frac{d \rho_{\mathrm{gw}} / \rho_{c}}{d \ln f}
$$

This can be interpreted as the fraction of the closure energy density that is in random gravitational waves between the frequency $f$ and $e \times f$. If the source of radiation is scale-free (which means that there is no preferred length or time scale in the process), then it will produce a power-law spectrum, i.e., one in which $\Omega_{\mathrm{gw}}(f)$ depends on a power of $f$. Inflation, as we describe below, predicts a flat energy spectrum, one in which $\Omega_{\mathrm{gw}}$ is essentially independent of frequency [27].

The energy in the cosmological background is, of course, related to the spectral density of the noise that the background would produce in a gravitational wave detector. Since we describe the gravitational wave noise in terms of amplitude rather than energy, there are scaling factors involving the frequency between the two. An isotropic gravitational wave background incident on an interferometric detector will induce a strain spectral noise density equal to [359, 27]

$$
S_{\mathrm{gw}}(f)=\frac{3 H_{0}^{2}}{10 \pi^{2}} f^{-3} \Omega_{\mathrm{gw}}(f) .
$$

Note that the explicit dependence on frequency is $f^{-3}$ : two factors come from the relation of energy and squared-strain, and one factor from the fact that $\Omega_{\mathrm{gw}}$ is an energy distribution per unit logarithmic frequency. Note also that there are no explicit factors of $c$ or $G$ needed in this formula if one wants to work in nongeometrized units.

If we scale $H_{0}$ by $h_{100}=H_{0} /\left(100 \mathrm{~km} \mathrm{~s}^{-1} \mathrm{Mpc}^{-1}\right)$, and we note that $100 \mathrm{~km} \mathrm{~s}^{-1} \mathrm{Mpc}^{-1}=$ $3.24 \times 10^{-18} \mathrm{~s}^{-1}$, then this equation implies that the strain noise is

$$
S_{\mathrm{gw}}^{1 / 2}=5.6 \times 10^{-22} \mathrm{~Hz}^{-1 / 2} \Omega_{\mathrm{gw}}^{1 / 2}\left(\frac{f}{100 \mathrm{~Hz}}\right)^{-3 / 2} h_{100} .
$$

\subsubsection{Observations with gravitational wave detectors}

To be observed by a single gravitational wave detector, the gravitational wave noise must be larger than the instrumental noise. This is a bolometric method of detection of the background, and it requires great confidence in the understanding of the detector, in order to believe that the observed noise is external. This is how the cosmic microwave background was originally discovered in a radio telescope by Penzias and Wilson.

Living Reviews in Relativity

http: //www . livingreviews . org/lrr-2009-2 
If there are two detectors, then one may be able to get better sensitivity by cross-correlating their output, as mentioned in Section 4.7.3 above. This works best when the two detectors are close enough together to respond to the same random wave field. Even when they are separated, however, they are correlated well at lower frequencies.

From Equation 135 and the discussion in Section 4.7.3 it is straightforward to deduce that two co-located detectors, each with spectral noise density $S_{h}$ and fully uncorrelated instrumental noise, observing over a bandwidth $f$ at frequency $f$ for a time $T$, can detect a stochastic background with energy density

$$
\Omega_{\mathrm{gw}}^{1 / 2} h_{100}=\left(\frac{S_{h}^{1 / 2}}{3.1 \times 10^{-18} \mathrm{~Hz}^{-1 / 2}}\right)\left(\frac{f}{10 \mathrm{~Hz}}\right)^{5 / 4}\left(\frac{T}{3 \mathrm{yrs}}\right)^{-1 / 4} .
$$

The two LIGO detectors (separated by about $10 \mathrm{~ms}$ in light-travel time) are reasonably well placed for performing such correlations, particularly when upgrades push their lower frequency limit to $20 \mathrm{~Hz}$ or less. Two co-located first-generation LIGO instruments operating at $100 \mathrm{~Hz}$ could, in a one-year correlation, reach a sensitivity of $\Omega_{\mathrm{gw}} \sim 1.7 \times 10^{-8}$. But the separation of the actual detectors takes its toll at this frequency, so that they can actually only reach $\Omega_{\mathrm{gw}} \sim 10^{-6}$. Advanced LIGO may improved this by two or three orders of magnitude, going well below the nucleosynthesis bound. The third-generation instrument ET, with instrumental noise as shown in Figure 5, can go even deeper. Two co-located ETs, observing at $10 \mathrm{~Hz}$ for three years, could reach $\Omega_{\mathrm{gw}} \sim 10^{-12}$. At this frequency the detectors could be as far apart as $5000 \mathrm{~km}$ without a substantial loss in correlation sensitivity. The numbers given here are reflected in the curves in Figure 5.

Correlation searches are also possible between resonant detectors or between one resonant and one interferometric detector [53]. This has been implemented with bar detectors [52] and between LIGO and the ALLEGRO bar detector [391].

LISA does not gain by a simple correlation between any two of its independent interferometers, since they share a common arm, which contributes common noise that competes with that of the background. A gravitational wave background of $\Omega_{\mathrm{gw}} \sim 10^{-10}$ would compete with LISA's expected instrumental noise. However, using all three interferometers together can improve things for LISA at low frequencies, assuming that the LISA instrumental noise is well behaved [194]. This might enable LISA to go below $10^{-11}$.

\subsubsection{Observations with pulsar timing}

Other less-direct methods are also being used to search for primordial gravitational waves. As we saw in Section 4.4.2, pulsar timing can, in principle, detect gravitational-wave-induced fluctuations in the arrival times of pulses. Millisecond pulsars are such stable clocks when averaged over years of observations that they are being used to search for gravitational waves with periods longer than one year. A single pulsar can set limits on a stochastic background by removing the slow spindown and looking for random timing residuals. Although one would never have enough confidence in the stability of a single pulsar to claim a detection, this sets upper limits in the important frequency range below that accessible to man-made instruments. The best such limits are on pulsar PSR B1855+09, with an upper limit (at $90 \%$ confidence) of $\Omega_{\mathrm{gw}}<4.8 \times 10^{-9}$ at $f=4.4 \mathrm{nHz}$ [212].

Arrays of pulsars offer the possibility of cross-correlating their fluctuations, which makes it possible to distinguish between intrinsic variability and gravitational-wave-induced variability. Pulsars are physically separated by much more than a wavelength of the gravitational waves even with periods of $10 \mathrm{yrs}$, so that the correlated fluctuations come from the wave amplitudes at Earth. It will soon be possible to monitor many pulsars simultaneously with multibeam instruments, as mentioned in Section 4.4.2. This method could push the limits on $h_{c} \equiv\left(f S_{\mathrm{gw}}\right)^{1 / 2}$ [cf. Equation (134)] down to $10^{-16}$ at $10 \mathrm{nHz}$ [223], which translates into a limit on $\Omega_{\mathrm{gw}}$ of around $10^{-12}$. 


\subsubsection{Observations using the cosmic microwave background}

Observations of the cosmic microwave background (CMB) may in fact make the first detections of stochastic (or any other!) gravitational waves. The temperature fluctuations first detected by the Cosmic Background Explorer (COBE) [342] and more recently measured with great precision by the Wilkinson Microwave Anisotropy Probe (WMAP) [72] are produced by both density perturbations and long-wavelength gravitational waves in the early universe (see the next Section 8.2.1). Inflation suggests that the gravitational wave component may be almost as large as the density component, but it can only be separated from the density perturbations by looking at the polarization of the cosmic microwave background [215]. WMAP made the first measurements of polarization [280], but it did not have the sensitivity to see the weak imprint of gravitational waves, which appears in the B-component of the polarization, the part that is divergence-free on the whole sky. The best limits on the B-component so far (early 2008) have been made by the QUaD ${ }^{13}$ detector [300], a cryogenic detector that operated for three seasons in Antarctica. These have not yet shown any evidence for gravitational waves. Results are expected soon from the Background Imaging of Cosmic Extragalactic Polarization (BICEP) detector, also in Antarctica [214]. The next satellite to study the microwave background will be Planck, due for launch by the European Space Agency in 2009 [154].

The gravitational waves detectable in the CMB have wavelengths a good fraction of the horizon size at the time of decoupling, and today they have been redshifted to much longer wavelengths. They are, therefore, much lower frequency than the radiation that would be observed directly by LISA or ground-based detectors, or even by pulsar timing.

\subsection{Origin of a random background of gravitational waves}

\subsubsection{Gravitational waves from the Big Bang}

Gravitational waves have traveled almost unimpeded through the universe since they were generated. The cosmic microwave background [72] is a picture of the universe at a time $3 \times 10^{5}$ yrs after the Big Bang, and studies of nucleosynthesis [346] (how the primordial hydrogen, helium, deuterium, and lithium were created) reveal conditions in the universe a few minutes after the Big Bang. Gravitational waves, on the other hand, were produced at times earlier than $10^{-24} \mathrm{~s}$ after the Big Bang. Observing this background would undoubtedly be one of the most important measurements that gravitational wave astronomy could make. It would provide a test of inflation, and it would have the potential to give information about the fundamental interactions of physics at energies far higher than we can reach with accelerators.

The most well-defined predictions about the energy in the cosmological gravitational wave background come from inflationary models. Inflation is an attractive scenario for the early universe because, among other things, it provides a natural mechanism for producing the initial density perturbations that evolved into galaxies and galaxy clusters as the universe expands. These perturbations start out as quantum fluctuations in the (hypothetical) scalar inflaton field that is responsible for the inflationary expansion of the universe. The fluctuations are parametrically amplified by the expansion $[182,262,27]$ and lead to fluctuations in the density of normal matter after inflation ends.

Several strands of evidence - among them the statistical distribution of density perturbations seen in the cosmic microwave background (most recently by WMAP [343]), the present distribution of galaxies [229], and numerical simulations of structure formation in the early universe [286] - are fully consistent with the now-standard model of a universe dominated by dark energy and whose

\footnotetext{
${ }^{13} \mathrm{QUaD}$ stands for QUEST (Q and U Extragalactic Survey Telescope) at DASI (Degree Angular Scale Interferometer).
}

Living Reviews in Relativity

http: //www. livingreviews.org/lrr-2009-2 
matter density is dominated by some kind of cold (i.e., massive) dark matter particles [350] with density perturbations consistent with those that inflation could have produced.

The scalar inflaton fluctuations are accompanied by tensor quantum fluctuations in the gravitational field that similarly get amplified by inflation and form a random background [26, 27]. Different models of inflation make different predictions about the relative strength of the scalar and tensor components.

Although inflation is in excellent agreement with observation, other mechanisms in the early universe may have led to the additional production of gravitational waves. Defects that arise from symmetry breaking as the presumed early unified interactions separate from one another can lead to cosmic strings [379], which can produce both a continuous observable gravitational wave background [102] and characteristic isolated bursts of gravitational waves [140, 141, 142]. String theory [93, 99] and brane theory [192, 248] may also provide mechanisms for generating observable radiation.

The various models usually predict significantly different spectra for background radiation. Standard inflationary models predict that the spectrum of $\Omega_{\mathrm{gw}}$ should be nearly flat, independent of frequency, but variants exist that allow a spectrum that rises with frequency (positive spectral index) or falls. Symmetry-breaking and brane model cosmologies can make very different predictions, even leading to narrow spectral features. It is, therefore, important to measure the spectrum at as many frequencies as possible. Limits on power at one frequency (such as at the very lowfrequency end in the cosmic microwave background) do not necessarily predict the power at other frequencies (such as at ground-based frequencies, a factor $10^{20}$ times higher).

It is even possible that there will be a feature in the spectrum in the observing band of groundbased or space-based detectors. In standard cosmologies, the radiation observable by LISA $(1 \mathrm{mHz})$ had a wavelength comparable to the (then) horizon size at around the time when the temperature of the universe was equal to the electroweak symmetry-breaking energy. If electroweak symmetry breaking led to a first-order phase transition, where density fluctuations occurred on the length scale of the typical symmetry domain size, then it is likely that these density fluctuations produced gravitational waves with wavelengths of the size of the horizon, which would be in the LISA band today [253]. Detection of this radiation would have deep implications for fundamental physics.

The other expected phase transition is the GUTs (Grand Unified Theory) transition, whose energy might have been $10^{13}$ times higher. Any gravitational radiation from this transition today would then be at a frequency $10^{13}$ times that from the electroweak transition, i.e., at centimeter wavelengths. This is one motivation for building microwave-based table-top detectors aimed at high frequencies [126]. For this radiation to be observable by standard interferometers, the GUTs transition would have to have an energy $10^{7}$ times smaller than expected, i.e., around $10^{9} \mathrm{GeV}$. We shall have to wait for observations at these frequencies to tell us if it is there!

In addressing the possibility of new physics, observation of gravitational waves in the cosmic microwave background would play a unique role. These waves originated long after nucleosynthesis, at energies where physics is presumably well understood. They would, therefore, normalize the amount of power in the initial tensor perturbations. Then observations at higher frequencies can use this normalization to measure the excess energy due to any exotic effects due to string theory, phase transitions, or other unknown physics [183, 99].

Pulsar timing arrays (see Section 8.1.3) will also be used to search for a CGWB at frequencies of a few nanoHertz. As for the microwave background, the physics of the universe when gravitational waves at these frequencies originated is well understood, so they could be used to normalize the spectrum. If the power at pulsar frequencies and that in the microwave background are not consistent, then this could indicate something about the conditions in the universe before inflation began.

The predicted spectrum from inflation, strings, and symmetry breakings is highly nonthermal. Any thermal radiation produced in the Big Bang (for example, if, hypothetically, there was some 
kind of equipartition between gravitational degrees of freedom and other fields in the initial data at the singularity, whatever that might mean!) would have been redshifted away to unobservability by the subsequent inflationary expansion. If inflation did not in fact occur, then this radiation today would have a temperature only a little below that of the cosmological microwave background. So far no instrument has been proposed that would be sensitive to this radiation, but its detection would presumably be inconsistent with inflation.

\subsubsection{Astrophysical sources of a stochastic background}

After galaxy formation, it is possible that many systems arose that have been radiating gravitational waves in the bands observable by pulsar timing, LISA, and ground-based detectors. There are likely to be strong extra-galactic backgrounds in the LISA band from compact binary systems, which would limit searches for a CGWB [325] by LISA, even if the sensitivity were better. At lower frequencies, even down to pulsar timing frequencies, black hole binaries may make the strongest background, while at frequencies above the LISA band (i.e., above $0.1 \mathrm{~Hz}$ ) the universe should be relatively free of serious backgrounds [158, 365].

In the LISA band our galaxy is a strong source of backgrounds [190]. This presents a serious confusion noise in searching for other sources at frequencies below $1 \mathrm{mHz}$. It should be possible to distinguish this from a CGWB by its intrinsic anisotropy [366].

\subsection{Cosmography: gravitational wave measurements of cosmological pa- rameters}

Since inspiral signals are standard candles [329], as described in Section 6, observations of massive black hole coalescences at cosmological distances by space-based detectors can facilitate an accurate determination of the distance to the source. Our earlier expressions for the chirp waveform can be generalized to the cosmological case (a source at redshift $z$ ) by multiplying all masses by $1+z$ and by replacing the physical distance $D$ by the cosmological luminosity distance $D_{L}$ [226]. If the wave amplitude, frequency, and chirp rate of the binary can be measured, then its luminosity distance can be inferred. It is not, however, possible to infer the redshift $z$ from the observed signal: the scale-invariance of black hole solutions means that a signal with a redshift of two and a chirp mass $\mathcal{M}$ looks identical to a signal with no redshift and a chirp mass of $\mathcal{M} / 3$. To use these distance measures for cosmography, one has to obtain redshifts of the host galaxies.

Before considering how this might be done, we should ask about the accuracy with which the distance can be measured. The relative error in the distance is dominated by the relative error in the measurement of the intrinsic amplitude of the gravitational wave, because the masses will normally be much more accurately measured (by fitting the evolving phase of the signal) than the amplitude. Several factors contribute to the amplitude uncertainty:

- Signal-to-noise ratio. The intrinsic measurement uncertainty in the amplitude of the detector's response is simply the inverse of the SNR. Since LISA can have an SNR of several thousand when it observes an SMBH coalescence at high redshift, LISA has great potential for cosmography.

- Position error. From the detector response one must infer the intrinsic amplitude of the wave, which means projecting it on the antenna pattern of the detectors. This requires a knowledge of the source position, and this will be potentially a bigger source of uncertainty because the sensitivity of LISA depends on the location of the source in its antenna pattern. Recent work [232, 58] has shown that LISA may be able to achieve position accuracies between one and ten arcminutes. At, say, three arcminutes error, the amplitude uncertainty will be of order $0.1 \%$. This error can be reduced to the SNR-limited error if the source can

Living Reviews in Relativity

http: //www. livingreviews.org/lrr-2009-2 
be identified. Although the coalescence of two SMBHs itself may not have an immediate effect on the visible light from a galaxy, the host galaxy might be identifiable either because it shows great irregularity (mergers of black holes follow from mergers of galaxies) or because some years after the merger an X-ray source turns on (accretion will be disrupted by the tidal forces of the orbiting black holes, but will start again after they merge) [257]. Other effects that might lead to an identification include evidence that stars have been expelled from the core of a galaxy, fossil radio jets going in more than two directions from a common center, and evidence for accretion having stopped in the recent past.

- Microlensing. If the source is at a redshift larger than one, as we can expect for LISA, then random microlensing can produce a magnification or demagnification on the order of a few percent $[195,131]$. The measured intrinsic amplitude then does not match the amplitude that the signal would have in an ideal smooth cosmology.

The relatively small error boxes within which the LISA coalescences can be localized are promising for identifications, especially if the X-ray indicators mentioned above pick out the host in the error box. These factors and their impact on cosmography measurements have been examined in detail by Holz and Hughes [195], who coined the term "standard siren" for the chirp sources whose distance can be determined by gravitational wave measurements. The potential for cosmographic measurements by advanced ground-based detectors have been considered in a further paper by the same authors and collaborators [131]. Nearby coalescences and IMRIs should provide an accurate determination of the Hubble Constant [204, 250]. Perhaps the most interesting measurement will be to characterize the evolution of the dark energy, which is usually characterized by inserting a parameter $w$ in the equation of state of dark energy, $p=w \rho$. If $w=-1$, then the dark energy is equivalent to a cosmological constant [108] and the energy density will be the same at all epochs. If $w>-1$, the dark energy is an evolving field whose energy density diminishes in time. According to [131], gravitational wave measurements have the potential to measure $w$ to an accuracy better than 10\% (for advanced ground-based detectors) and around 4\% (for LISA). The accuracy with which parameters can be measured improves greatly when one includes in the computation of the covariance matrix the harmonics of the binary inspiral signal that is normally neglected [374]. Arun et al. [48] have shown that the source location in the sky can be greatly improved when the signal harmonics (up to fifth harmonic) are included, which further helps in measuring the parameter $w$ even better. 


\section{Conclusions and Future Directions}

The development of gravitational wave detectors to their present capability has required patience, ingenuity, and dedication by an entire generation of experimental physicists. No less dedication and vision have been required by scientific funding organizations of a half-dozen nations and two major space agencies. The initial data runs of the LIGO and VIRGO detectors at their first sensitivity goals (bursts with amplitudes of $10^{-21}$ ) have not so far yielded any detections, but this is certainly not surprising. The operation of these detectors at this sensitivity level has demonstrated that the technology is understood, and the analysis of the data has provided important early experience and the opportunity to organize the efforts into the LSC and VIRGO collaborations. As the detectors are upgraded during the period 2008-2014, the first detection could occur at any time; if the advanced detectors do not make early detections, then there will inevitably be serious questions about general relativity. The field of gravitational wave detection has never before been at the point where it could test the fundamental theory.

Once the first detection is made, there will be increasing emphasis on the fundamental physics and astrophysics that will follow from further detections. As we have discussed in this review, one can look forward soon thereafter to a detailed comparison of black hole mergers with theory, to exploring the relationship between compact-object mergers and gamma-ray bursts, to using this association to make a precise and calibration-free measurement of the Hubble constant, and to population studies of neutron stars and black holes. In this early phase of gravitational wave astronomy there are very exciting (but less certain) potential observations: an unexpectedly strong cosmological background, which would revolutionize early-universe physics; the detection of mass asymmetry or normal-mode oscillations of rotating neutron stars, either of which would for the first time probe the interior physics of these complex objects and would help unravel the mystery of the pulsar phenomenon; the first studies of the interior core dynamics of a supernova, if one happens to occur nearby; the detection of populations of compact dark objects, like cosmic strings or small black holes; the discovery of exceptionally-massive black holes, around $100 M_{\odot}$; or the association of gravitational wave events with transient phenomena other than gamma-ray bursts, such as transient radio bursts.

When LISA is launched, the physics and astrophysics consequences become even richer. LISA will study black hole mergers during the early phases of galaxy formation, exploring the mysterious link between the two. It will map in detail black hole spacetimes and verify the black-hole uniqueness and area theorems of general relativity. It is likely to map the history of the expansion of the universe through measuring the distances to massive black hole mergers, and from that look for evidence that the dark energy has been evolving with time. It will discover every short-period binary system in our galaxy, calibrating white-dwarf masses, mapping their mass distribution, determining the population of neutron stars in binaries. As with ground-based detectors, LISA might make other discoveries that are harder to predict, such as a cosmological background, cosmic strings, intermediate-mass black holes, even $g$-mode oscillations of the sun. LISA has enough sensitivity to be able to make discoveries even of sources for which there are no signal models to aid data analysis. And if LISA does not see its verification binary sources, that will be fatal for general relativity.

Gravitational wave detections may also come from other technologies, such as pulsar timing searches or observations of the cosmic microwave background. The spectrum of gravitational waves is enormous, and present technologies can explore only a tiny fraction of it. Beyond the LISA timeframe, say after 2020, new technologies may come into the field and make possible detectors that extend the ground-based detection band to lower frequencies (such as the Einstein Telescope project), observing in space in the $0.1 \mathrm{~Hz}$ band, going up to megaHertz frequencies.

The present review has attempted to give a good overview of the science that can be done with gravitational waves, but it is certainly not complete. Future revisions are planned to add more

Living Reviews in Relativity

http: //www . livingreviews . org/lrr-2009-2 
on LISA, more on data analysis issues, and considerably more on detectors that might go beyond Advanced LIGO and VIRGO. This is a field that is developing rapidly. For example, the launch of LISA is 10 years away (at the time of writing, 2008), but already the scientific literature contains many hundreds of refereed papers on LISA science and technology, and every second year there is a major international symposium on the subject. This is probably unprecedented among space missions. Living Reviews in Relativity is planning to release a suite of articles in the near future on LISA, which will cover cosmology, tests of general relativity, galactic astrophysics, black hole astrophysics, and observations of low-frequency gravitational wave sources with LISA. Until the next revision, readers interested in keeping up with the field should also consult the proceedings of the regular conferences on gravitational waves: the Amaldi meetings, GWDAW (Gravitational Wave Data Analysis Workshops), GWADW (Gravitational Wave Advanced Detectors Workshops), and the LISA Symposium. 


\section{Acknowledgements}

We would like to thank all authors who granted permission to reproduce their figures. We greatly acknowledge our GEO colleagues, from whom we have learned so much over the years. Finally, this article would not have seen the light of day without the insistence of Professor Bala Iyer; we are thankful to him for his patience and encouragement. 


\section{References}

[1] Abbott, B. et al. (LIGO Scientific Collaboration), "Analysis of First LIGO Science Data for Stochastic Gravitational Waves", Phys. Rev. D, 69, 122004, (2004). [gr-qc/0312088]. 8

[2] Abbott, B. et al. (LIGO Scientific Collaboration), "Analysis of LIGO data for gravitational waves from binary neutron stars", Phys. Rev. D, 69, 122001, (2004). [gr-qc/0308069]. 4.7.1, 5.1.3.1, 5.1.3.3

[3] Abbott, B. et al. (LIGO Scientific Collaboration), "Detector description and performance for the first coincidence observations between LIGO and GEO", Nucl. Instrum. Methods A, 517, 154-179, (2004). [gr-qc/0308043]. 1

[4] Abbott, B. et al. (LIGO Scientific Collaboration), "First all-sky upper limits from LIGO on the strength of periodic gravitational waves using the Hough transform", Phys. Rev. D, 72, 102004, (2005). [DOI], [gr-qc/0508065]. 5.1.3.2

[5] Abbott, B. et al. (LIGO Scientific Collaboration), "Limits on gravitational wave emission from selected pulsars using LIGO data", Phys. Rev. Lett., 94, 181103, (2005). [DOI], [grqc/0410007]. 5.1.3.2

[6] Abbott, B. et al. (LIGO Scientific Collaboration), "Search for gravitational waves from galactic and extra- galactic binary neutron stars", Phys. Rev. D, 72, 082001, (2005). [grqc/0505041]. 4.7.1, 5.1.3.1, 5.1.3.3

[7] Abbott, B. et al. (LIGO Scientific Collaboration), "Search for gravitational waves from primordial black hole binary coalescences in the galactic halo", Phys. Rev. D, 72, 082002, (2005). [gr-qc/0505042]. 4.7.1, 5.1.3.1

[8] Abbott, B. et al. (LIGO Scientific Collaboration), "Search for gravitational waves from binary black hole inspirals in LIGO data", Phys. Rev. D, 73, 062001, (2006). [gr-qc/0509129]. 4.7.1, 5.1.3.1

[9] Abbott, B. et al. (LIGO Scientific Collaboration), "Search for gravitational-wave bursts in LIGO data from the fourth science run", Class. Quantum Grav., 24, 5343-5370, (2007). [DOI], [arXiv:0704.0943]. 5.2

[10] Abbott, B. et al. (LIGO Scientific Collaboration), "Upper limits on gravitational wave emission from 78 radio pulsars", Phys. Rev. D, 76, 042001, (2007). [DOI], [gr-qc/0702039]. 4.3.1, 5.1 .3 .2

[11] Abbott, B. et al. (LIGO Scientific Collaboration), "All-sky search for periodic gravitational waves in LIGO S4 data", Phys. Rev. D, 77, 022001, (2008). [DOI], [arXiv:0708.3818]. 5.1.3.2, 7.4

[12] Abbott, B. et al. (LIGO Scientific Collaboration), "Beating the spin-down limit on gravitational wave emission from the Crab pulsar", Astrophys. J. Lett., 683, L45-L49, (2008). [arXiv:0805.4758]. 3.3, 4.3.1, 5.1.3.2

[13] Abbott, B. et al. (LIGO Scientific Collaboration), "The Einstein(AT)Home search for periodic gravitational waves in LIGO S4 data", Phys. Rev. D, 79, 022001, (2008). [arXiv:0804.1747]. 5.1.3.2

[14] Abbott, B. et al. (LIGO Scientific Collaboration), "Search for gravitational waves associated with 39 gamma-ray bursts using data from the second, third, and fourth LIGO runs", Phys. Rev. D, 77, 062004, (2008). [arXiv:0709.0766]. 7.4 
[15] Abbott, B. et al. (LIGO Scientific Collaboration), "Search of S3 LIGO data for gravitational wave signals from spinning black hole and neutron star binary inspirals", Phys. Rev. D, 78, 042002, (2008). [DOI], [arXiv:0712.2050]. 5.1.3.1

[16] Abbott, B. et al. (LIGO Scientific Collaboration), and Hurley, K., "Implications for the Origin of GRB 070201 from LIGO Observations", Astrophys. J., 681, 1419-1430, (2008). [arXiv:0711.1163]. 7.4

[17] Abbott, B. et al. (LIGO Scientific Collaboration \& ALLEGRO Collaboration), "First crosscorrelation analysis of interferometric and resonant-bar gravitational-wave data for stochastic backgrounds", Phys. Rev. D, 76, 022001, 1-17, (2007). [gr-qc/0703068]. 4.1

[18] Abramovici, A., Althouse, W.E., Drever, R.W.P., Gürsel, Y., Kawamura, S., Raab, F.J., Shoemaker, D.H., Sievers, L., Spero, R.E., Thorne, K.S., Vogt, R.E., Weiss, R., Whitcomb, S.E., and Zucker, M.E., "LIGO: The Laser Interferometer-Gravitational Wave Observatory", Science, 256, 325-333, (1992). [DOI]. 1

[19] Acernese, F. et al. (Virgo Collaboration), "The Virgo status", Class. Quantum Grav., 23, S635-S642, (2006). [DOI]. 1

[20] Acernese, F. et al. (Virgo Collaboration), "Status of Virgo detector", Class. Quantum Grav., 24, S381-S388, (2007). [DOI]. 4.3.1

[21] Aguiar, O.D., Andrade, L.A., Barroso, J.J., Bortoli, F., Carneiro, L.A., Castro, P.J., Costa, C.A., Costa, K.M.F., de Araujo, J.C.N., de Lucena, A.U., de Paula, W., de Rey Neto, E.C., de Souza, S.T., Fauth, A.C., Frajuca, C., Frossati, G., Furtado, S.R., Magalháes, N.S., Marinho Jr, R.M., Matos, E.S., Melo, J.L., Miranda, O.D., Oliveira Jr, N.F., Paleo, B.W., Remy, M., Ribeiro, K.L., Stellati, C., Velloso Jr, W.F., and Weber, J., "The Brazilian gravitational wave detector Mario Schenberg: progress and plans", Class. Quantum Grav., 22, S209-S214, (2005). [DOI]. 4.1

[22] Ajith, P. et al., "Phenomenological template family for black-hole coalescence waveforms", Class. Quantum Grav., 24, S689-S700, (2007). [DOI], [arXiv:0704.3764]. 6.5.2, 6.5.2

[23] Akutsu, T., Kawamura, S., Nishizawa, A., Arai, K., Yamamoto, K., Tatsumi, D., Nagano, S., Nishida, E., Chiba, T., Takahashi, R., Sugiyama, N., Fukushima, M., Yamazaki, T., and Fujimoto, M., "Search for a stochastic background of 100-MHz gravitational waves with laser interferometers", Phys. Rev. Lett., 101, 101101, (2008). [arXiv:0803.4094]. 4.3.2

[24] Albert Einstein Institute, "Laser Interferometer Space Antenna", project homepage. URL (cited on 08 November 2007):

http://www.lisa.aei-hannover.de/. 4.4 .3

[25] Alcock, C., Allsman, R.A., Alves, D., Axelrod, T.S., Becker, A.C., Bennett, D.P., Cook, K.H., Freeman, K.C., Griest, K., Guern, J., Lehner, M.J., Marshall, S.L., Peterson, B.A., Pratt, M.R., Quinn, P.J., Rodgers, A.W., Stubbs, C.W., Sutherland, W., and Welch, D.L. (The MACHO Collaboration), "The MACHO Project: LMC Microlensing Results from the First Two Years and the Nature of the Galactic Dark Halo", Astrophys. J., 486, 697-726, (1997). [astro-ph/9606165]. 7.2.2

[26] Allen, B., "Stochastic gravity-wave background in inflationary-universe models", Phys. Rev. $D, 37,2078-2085,(1988)$. [DOI]. 8.2.1 
[27] Allen, B., "The Stochastic Gravity-Wave Background: Sources and Detection", in Marck, J.A., and Lasota, J.-P., eds., Relativistic Gravitation and Gravitational Radiation, Proceedings of the Les Houches School of Physics, held in Les Houches, Haute Savoie, 26 September -6 October, 1995, Cambridge Contemporary Astrophysics, pp. 373-418, (Cambridge University Press, Cambridge, 1997). 3.6, 4.7.3, 8, 8.1.1, 8.2.1

[28] Allen, B., " $\chi^{2}$ time-frequency discriminator for gravitational wave detection", Phys. Rev. D, 71, 062001, (2005). [DOI], [gr-qc/0405045]. 4.8, 5.1.3.3, 5.1.3.3, 5.1.3.3

[29] Allen, Z.A. et al. (International Gravitational Event Collaboration), "First Search for Gravitational Wave Bursts with a Network of Detectors", Phys. Rev. Lett., 85, 5046-5050, (2000). [astro-ph/0007308]. 5

[30] Amaro-Seoane, P., "Gravitational waves from coalescing massive black holes in young dense clusters", in Merkowitz, S.M., and Livas, J.C., eds., Laser Interferometer Space Antenna: Sixth International LISA Symposium, Greenbelt, Maryland, U.S.A., 19-23 June 2006, AIP Conference Proceedings, vol. 873, pp. 250-256, (American Institute of Physics, Melville, NY, 2006). [DOI], [astro-ph/0610479]. 3.4.6

[31] Amaro-Seoane, P., and Freitag, M., "Intermediate-mass black holes in colliding clusters: Implications for lower-frequency gravitational-wave astronomy", Astrophys. J., 653, L53L56, (2006). [astro-ph/0610478]. 3.4.6

[32] Amaro-Seoane, P., Gair, J.R., Freitag, M., Coleman, M.M., Mandel, I., Cutler, C.J., and Babak, S., "Intermediate and Extreme Mass-Ratio Inspirals - Astrophysics, Science Applications and Detection using LISA", Class. Quantum Grav., 24, R113-R170, (2007). [astro$\mathrm{ph} / 0703495]$. 3.4.6, 7.2.4

[33] Anderson, W.G., Brady, P.R., Creighton, J.D.E., and Flanagan, É.É., "A power filter for the detection of burst sources of gravitational radiation in interferometric detectors", Int. J. Mod. Phys. D, 9, 303-307, (2000). [gr-qc/0001044]. 5.2

[34] Andersson, N., "A new class of unstable modes of rotating relativistic stars", Astrophys. J., 502, 708-713, (1998). [gr-qc/9706075]. 7, 7.3.4.2

[35] Andersson, N., and Comer, G.L., "Relativistic Fluid Dynamics: Physics for Many Different Scales", Living Rev. Relativity, 10, lrr-2007-1, (2007). URL (cited on 03 September 2007): http://www.livingreviews . org/lrr-2007-1. 7.3.3, 7.3.4.2

[36] Andersson, N., and Kokkotas, K.D., "Towards gravitational wave asteroseismology", Mon. Not. R. Astron. Soc., 299, 1059-1068, (1998). [gr-qc/9711088]. 7.3.3

[37] Andersson, N., Kokkotas, K.D., and Schutz, B.F., "Gravitational radiation limit on the spin of young neutron stars", Astrophys. J., 510, 846-853, (1999). [astro-ph/9805225]. 7.3.4.2

[38] Apostolatos, T.A., "Search templates for gravitational waves from precessing, inspiraling binaries", Phys. Rev. D, 52, 605-620, (1995). [DOI]. 5.1.3.1

[39] Aricebo Observatory, "NanoGrav", project homepage. URL (cited on 19 May 2008): http://arecibo.cac.cornell.edu/arecibo-staging/nanograv/. 4.4.2

[40] Armstrong, J.W., "Low-Frequency Gravitational Wave Searches Using Spacecraft Doppler Tracking", Living Rev. Relativity, 9, lrr-2006-1, (2006). URL (cited on 03 September 2007): http://www.livingreviews.org/lrr-2006-1. 4.2, 4.4.1 
[41] Arnaud, N., Barsuglia, M., Bizouard, M.-A., Brisson, V., Cavalier, F., Davier, M., Hello, P., Kreckelbergh, S., and Porter, E.K., "Coincidence and coherent data analysis methods for gravitational wave bursts in a network of interferometric detectors", Phys. Rev. D, 68, 102001, (2003). [gr-qc/0307100]. 4.7.1

[42] Arnaud, N., Barsuglia, M., Bizouard, M.-A., Canitrot, P., Cavalier, F., Davier, M., Hello, P., and Pradier, T., "Detection in coincidence of gravitational wave bursts with a network of interferometric detectors. I: Geometric acceptance and timing", Phys. Rev. D, 65, 042004, (2002). [DOI], [gr-qc/0107081]. 4.7.1

[43] Arons, J., "Magnetars in the Metagalaxy: An Origin for Ultra-High-Energy Cosmic Rays in the Nearby Universe", Astrophys. J., 589, 871-892, (2003). [astro-ph/0208444]. 7.4

[44] Arun, K.G., "Parameter estimation of coalescing supermassive black hole binaries with LISA", Phys. Rev. D, 74, 024025, (2006). [gr-qc/0605021]. 6.5.4, 6.5.4.1

[45] Arun, K.G., Iyer, B.R., Qusailah, M.S.S., and Sathyaprakash, B.S., "Probing the non-linear structure of general relativity with black hole mergers", Phys. Rev. D, 74, 024006, (2006). [DOI], [gr-qc/0604067]. 6.6.1, 6.6.1, 16

[46] Arun, K.G., Iyer, B.R., Qusailah, M.S.S., and Sathyaprakash, B.S., "Testing post-Newtonian theory with gravitational wave observations", Class. Quantum Grav., 23, L37-L43, (2006). [gr-qc/0604018]. 6.6.1, 6.6.1, 16

[47] Arun, K.G., Iyer, B.R., Sathyaprakash, B.S., and Sinha, S., "Higher harmonics increase LISA's mass reach for supermassive black holes", Phys. Rev. D, 75, 124002, (2007). [DOI], [arXiv:0704.1086]. 6.5.5

[48] Arun, K.G., Iyer, B.R., Sathyaprakash, B.S., Sinha, S., and Van Den Broeck, C., "Higher signal harmonics, LISA's angular resolution and dark energy", Phys. Rev. D, 76, 104016, (2007). [DOI], [arXiv:0707.3920]. 3.4.2, 6.5.5, 8.3

[49] Arun, K.G., Iyer, B.R., Sathyaprakash, B.S., and Sundararajan, P.A., "Parameter estimation of inspiralling compact binaries using 3.5 post-Newtonian gravitational wave phasing: The nonspinning case", Phys. Rev. D, 71, 084008, 1-16, (2005). [DOI], [gr-qc/0411146]. 12

[50] Astone, P., Babusci, D., Baggio, L., Bassan, M., Blair, D.G., Bonaldi, M., Bonifazi, P., Busby, D., Carelli, P., Cerdonio, M., Coccia, E., Conti, L., Cosmelli, C., D'Antonio, S., Fafone, V., Falferi, P., Fortini, P., Frasca, S., Giordano, G., Hamilton, W.O., Heng, I.S., Ivanov, E.N., Johnson, W.W., Marini, A., Mauceli, E., McHugh, M.P., Mezzena, R., Minenkov, Y., Modena, I., Modestino, G., Moleti, A., Ortolan, A., Pallottino, G.V., Pizzella, G., Prodi, G.A., Quintieri, L., Rocchi, A., Rocco, E., Ronga, F., Salemi, F., Santostasi, G., Taffarello, L., Terenzi, R., Tobar, M.E., Torrioli, G., Vedovato, G., Vinante, A., Visco, M., Vitale, S., and Zendri, J.P. (International Gravitational Event Collaboration), "Methods and results of the IGEC search for burst gravitational waves in the years 1997-2000", Phys. Rev. D, 68, 022001, 1-33, (2003). [astro-ph/0302482]. 4.1

[51] Astone, P., Babusci, D., Bassan, M., Bonifazi, P., Coccia, E., D’Antonio, S., Fafone, V., Giordano, G., Marini, A., Minenkov, Y., Modena, I., Modestino, G., Moleti, A., Pallottino, G.V., Pizzella, G., Quintieri, L., Rocchi, A., Ronga, F., Terenzi, R., and Visco, M., "The next science run of the gravitational wave detector NAUTILUS", Class. Quantum Grav., 19, 1911-1917, (2002). [DOI]. 1 
[52] Astone, P., Bassan, M., Bonifazi, P., Carelli, P., Coccia, E., Fafone, V., Frasca, S., Minenkov, Y., Modena, I., Modestino, P., Moleti, A., Pallottino, G.V., Pizzella, G., Terenzi, R., and Visco, M., "Upper limit at $1.8 \mathrm{kHz}$ for a gravitational-wave stochastic background with the ALTAIR resonant-mass detector", Astron. Astrophys., 343, 19, (1999). [ADS]. 8.1.2

[53] Astone, P., Lobo, A., and Schutz, B.F., "Coincidence experiments between interferometric and resonant bar detectors of gravitational waves", Class. Quantum Grav., 11, 2093-2112, (1994). [DOI]. 8.1.2

[54] Astone, P. et al. (IGEC-2 Collaboration), "Results of the IGEC-2 search for gravitational wave bursts during 2005", Phys. Rev. D, 76, 102001, (2007). [arXiv:0705.0688]. 4.1

[55] Australia Telescope National Facility, "ATNF Pulsar Catalogue", web interface to database. URL (cited on 19 May 2008):

http://www.atnf.csiro.au/research/pulsar/psrcat/. 7.3.6

[56] Australian National University, "ACIGA: Australian Consortium for Interferometric Gravitational Astronomy", project homepage. URL (cited on 08 November 2007):

http://www.anu.edu.au/Physics/ACIGA/. 4.3.1

[57] Babak, S., Fang, H., Gair, J.R., Glampedakis, K., and Hughes, S.A., "'Kludge' gravitational waveforms for a test-body orbiting a Kerr black hole", Phys. Rev. D, 75, 024005, (2007). [gr-qc/0607007]. 3.4.6

[58] Babak, S., Hannam, M., Husa, S., and Schutz, B.F., "Resolving Super Massive Black Holes with LISA", arXiv e-print, (2008). [arXiv:0806.1591]. 8.3

[59] Baggio, L., Bignotto, M., Bonaldi, M., Cerdonio, M., Conti, L., Falferi, P., Liguori, N., Marin, A., Mezzena, R., Ortolan, A., Poggi, A., Prodi, G.A., Salemi, F., Soranzo, G., Taffarello, L., Vedovato, G., Vinante, A., Vitale, S., and Zendri, J.P., "3-Mode Detection for Widening the Bandwidth of Resonant Gravitational Wave Detectors", Phys. Rev. Lett., 94, 241101, (2005). [gr-qc/0502101]. 2

[60] Baiotti, L., Giacomazzo, B., and Rezzolla, L., "Accurate evolutions of inspiralling neutronstar binaries: prompt and delayed collapse to black hole", Phys. Rev. D, 78, 084033, (2008). [DOI], [arXiv:0804.0594]. 7.3.2, 7.4

[61] Baker, J.G., Centrella, J., Choi, D.-I., Koppitz, M., and van Meter, J., "Gravitational-wave extraction from an inspiraling configuration of merging black holes", Phys. Rev. Lett., 96, 111102, (2006). [DOI], [gr-qc/0511103]. 6.5.2

[62] Baker, J.G., Centrella, J.M., Choi, D.-I., Koppitz, M., van Meter, J.R., and Miller, M.C., "Getting a kick out of numerical relativity", Astrophys. J. Lett., 653, L93-L96, (2006). [DOI], [astro-ph/0603204]. 6.5.2

[63] Balasubramanian, R., and Dhurandhar, S.V., "Estimation of parameters of gravitational wave signals from coalescing binaries", Phys. Rev. D, 57, 3408-3422, (1998). [DOI], [grqc/9708003]. 5.3.3, 6.5.4

[64] Balasubramanian, R., Sathyaprakash, B.S., and Dhurandhar, S.V., "Estimation of parameters of gravitational waves from coalescing binaries", Pramana, 45, L463-L470, (1995). [gr-qc/9508025]. 5.3, 5.3.2, 6.5.4

[65] Balasubramanian, R., Sathyaprakash, B.S., and Dhurandhar, S.V., "Gravitational waves from coalescing binaries: Detection strategies and Monte Carlo estimation of parameters", Phys. Rev. D, 53, 3033-3055, (1996). [gr-qc/9508011]. 5.3, 5.3.2, 6, 6.5.4 
[66] Barack, L., and Cutler, C., "Confusion Noise from LISA Capture Sources", Phys. Rev. D, 70, 122002, (2004). [gr-qc/0409010]. 6.6.2

[67] Barack, L., and Cutler, C., "LISA Capture Sources: Approximate Waveforms, Signal-toNoise Ratios, and Parameter Estimation Accuracy", Phys. Rev. D, 69, 082005, (2004). [grqc/0310125]. 3.4.6, 4.5.1

[68] Barack, L., and Cutler, C., "Using LISA EMRI sources to test off-Kerr deviations in the geometry of massive black holes", Phys. Rev. D, 75, 042003, (2007). [gr-qc/0612029]. 3.4.6, 6.6

[69] Baskaran, D., and Grishchuk, L.P., "Components of the gravitational force in the field of a gravitational wave", Class. Quantum Grav., 21, 4041-4061, (2004). [DOI], [gr-qc/0309058]. 4.2.1

[70] Bender, P.L., Brillet, A., Ciufolini, I., Cruise, A.M., Cutler, C., Danzmann, K., Fidecaro, F., Folkner, W.M., Hough, J., McNamara, P.W., Peterseim, M., Robertson, D., Rodrigues, M., Rüdiger, A., Sandford, M., Schäfer, G., Schilling, R., Schutz, B.F., Speake, C.C., Stebbins, R.T., Sumner, T.J., Touboul, P., Vinet, J.-Y., Vitale, S., Ward, H., and Winkler, W. (LISA Study Team), LISA. Laser Interferometer Space Antenna for the detection and observation of gravitational waves. An international project in the field of Fundamental Physics in Space. Pre-Phase A report. Second Edition, MPQ Reports, MPQ-233, (Max-Planck-Institut für Quantenoptik, Garching, 1998). Related online version (cited on 27 February 2009): ftp://ftp.ipp-garching.mpg.de/pub/grav/lisa/pdd. 4.5.1

[71] Bender, P.L., Ciufolini, I., Danzmann, K., Folkner, W.M., Hough, J., Robertson, D., Rüdiger, A., Sandford, M., Schilling, R., Schutz, B.F., Stebbins, R., Sumner, T., Touboul, P., Vitale, S., Ward, H., Winkler, W., Cornelisse, J., Hechler, F., Jafry, Y., and Reinhard, R., LISA. Laser Interferometer Space Antenna for the detection and observation of gravitational waves. A Cornerstone Project in ESA's long term space science programme "Horizon 2000 Plus". Pre-Phase A Report, December 1995, MPQ Reports, MPQ-208, (Max-Planck-Institut für Quantenoptik, Garching, 1996). Related online version (cited on 26 February 2009):

ftp://ftp.ipp-garching.mpg.de/pub/grav/lisa/ppa.ps.gz. Also see the Second Edition, MPQ-233. 1

[72] Bennett, C., Hill, R.S., Hinshaw, G., Nolta, M.R., Odegard, N., Page, L., Spergel, D.N., Weiland, J.L., Wright, E.L., Halpern, M., Jarosik, N., Kogut, A., Limon, M., Meyer, S.S., Tucker, G.S., and Wollack, E., "First-Year Wilkinson Microwave Anisotropy Probe (WMAP) Observations: Foreground Emission", Astrophys. J. Suppl. Ser., 148, 97, (2003). [astroph/0302208]. 8.1.4, 8.2.1

[73] Berti, E., Buonanno, A., and Will, C.M., "Estimating spinning binary parameters and testing alternative theories of gravity with LISA", Phys. Rev. D, 71, 084025, (2005). [DOI], [grqc/0411129]. 6.6.1, 6.6.1

[74] Berti, E., Buonanno, A., and Will, C.M., "Testing general relativity and probing the merger history of massive black holes with LISA", Class. Quantum Grav., 22, S943-S954, (2005). [gr-qc/0504017]. 6.6.1

[75] Berti, E., Cardoso, V., Gonzalez, J.A., Sperhake, U., Hannam, M., Husa, S., and Brügmann, B., "Inspiral, merger and ringdown of unequal mass black hole binaries: A multipolar analysis", Phys. Rev. D, 76, 064034, (2007). [DOI], [gr-qc/0703053]. 6.5.2 
[76] Berti, E., Cardoso, V., and Will, C.M., "Gravitational-wave spectroscopy of massive black holes with the space interferometer LISA", Phys. Rev. D, 73, 064030, (2006). [gr-qc/0512160]. $8,6.4,6.4,6.4,9,6.4$

[77] Bildsten, L., "Gravitational radiation and rotation of accreting neutron stars", Astrophys. J. Lett., 501, L89-L93, (1998). [astro-ph/9804325]. 3.3, 7, 7.3.5

[78] Blanchet, L., "Gravitational Radiation from Relativistic Sources", in Marck, J.-A., and Lasota, J.P., eds., Relativistic Gravitation and Gravitational Radiation, Proceedings of the Les Houches School of Physics, held in Les Houches, Haute Savoie, 26 September-6 October, 1995, pp. 33-66, (Cambridge University Press, Cambridge, 1997). [gr-qc/9607025]. 6.5.3

[79] Blanchet, L., "Gravitational Radiation from Post-Newtonian Sources and Inspiralling Compact Binaries", Living Rev. Relativity, 9, lrr-2006-4, (2006). URL (cited on 03 September 2007):

http://www.livingreviews.org/lrr-2006-4. 2.4, 6.5.3, 6.5.3.1, 6.5.5

[80] Blanchet, L., Damour, T., Esposito-Fares̀e, G., and Iyer, B.R., "Gravitational radiation from inspiralling compact binaries completed at the third post-Newtonian order", Phys. Rev. Lett., 93, 091101, (2004). [DOI], [gr-qc/0406012]. 6.5.3.1

[81] Blanchet, L., Damour, T., Esposito-Farèse, G., and Iyer, B.R., "Dimensional regularization of the third post-Newtonian gravitational wave generation from two point masses", Phys. Rev. D, 71, 124004, (2005). [DOI], [gr-qc/0503044]. 6.5.3.1

[82] Blanchet, L., Damour, T., Iyer, B.R., Will, C.M., and Wiseman, A.G., "GravitationalRadiation Damping of Compact Binary Systems to Second Post-Newtonian Order", Phys. Rev. Lett., 74, 3515-3518, (1995). [gr-qc/9501027]. 6.5.3

[83] Blanchet, L., and Sathyaprakash, B.S., "Signal analysis of gravitational wave tails", Class. Quantum Grav., 11, 2807-2831, (1994). [DOI]. 7.2

[84] Blanchet, L., and Sathyaprakash, B.S., "Detecting the tail effect in gravitational wave experiments", Phys. Rev. Lett., 74, 1067-1070, (1995). [DOI]. 6.6.1, 7.2

[85] Blanchet, L., and Schäfer, G., "Gravitational wave tails and binary star systems", Class. Quantum Grav., 10, 2699-2721, (1993). [DOI]. 6.6.1

[86] Bonaldi, M., Cerdonio, M., Conti, L., Falferi, P., Leaci, P., Odorizzi, S., Prodi, G.A., Saraceni, M., Serra, E., and Zendri, J.P., "Principles of wide bandwidth acoustic detectors and the single-mass dual detector", Phys. Rev. D, 74, 022003, (2006). [gr-qc/0605004]. 4.1

[87] Bose, S., Dhurandhar, S.V., and Pai, A., "Detection of gravitational waves using a network of detectors", Pramana, 53, 1125-1136, (1999). [gr-qc/9906064]. 4.7.1

[88] Boyle, M., Brown, D.A., Kidder, L.E., Mroué, A.H., Pfeiffer, H.P., Scheel, M.A., Cook, G.B., and Teukolsky, S.A., "High-accuracy comparison of numerical relativity simulations with post-Newtonian expansions", Phys. Rev. D, 76, 124038, (2007). [DOI], [arXiv:0710.0158]. 6.5.2, 6.5 .2

[89] Boyle, M., Lindblom, L., Pfeiffer, H., Scheel, M., and Kidder, L.E., "Testing the Accuracy and Stability of Spectral Methods in Numerical Relativity", Phys. Rev. D, 75, 024006, (2007). [DOI], [gr-qc/0609047]. 6.5.2 
[90] Brady, P.R., Creighton, T., Cutler, C., and Schutz, B.F., "Searching for periodic sources with LIGO", Phys. Rev. D, 57, 2101-2116, (1998). [gr-qc/9702050]. 5.1.3.2

[91] Bruce, A., and Romano, J.D., "Detecting a stochastic background of gravitational radiation: Signal processing strategies and sensitivities", Phys. Rev. D, 59, 102001, (1999). [DOI], [grqc/9710117]. 4.7.3

[92] Brügmann, B., Gonzalez, J.A., Hannam, M., Husa, S., and Sperhake, U., "Exploring black hole superkicks", Phys. Rev. D, 77, 124047, (2008). [DOI], [arXiv:0707.0135]. 6.5.2

[93] Brustein, R., Gasperini, M., Giovannini, M., and Veneziano, G., "Gravitational Radiation from String Cosmology", in Lemonne, J., Van der Velde, C., and Verbeure, F., eds., International Europhysics Conference on High Energy Physics (HEP95), Brussels, Belgium, July 27 -August 2, 1995, pp. 408-409, (World Scientific, Singapore; River Edge, NJ, 1996). [hep-th/9510081]. 8.2.1

[94] Buonanno, A., "Gravitational waves", in Bernardeau, F., Grojean, C., and Dalibard, J., eds., Particle Physics and Cosmology: The Fabric of Spacetime, Proceedings of the Les Houches Summer School, Session LXXXVI, 31 July-25 August 2006, pp. 3-52, (Elsevier, Amsterdam; Oxford, 2007). [arXiv:0709.4682]. 10

[95] Buonanno, A., Chen, Y., and Vallisneri, M., "Detection template families for precessing binaries of spinning compact binaries: Adiabatic limit", Phys. Rev. D, 67, 104025, (2003). [gr-qc/0211087]. Erratum-ibid. D74, 029904(E) (2006). 5.1.3.1

[96] Buonanno, A., and Damour, T., "Effective one-body approach to general relativistic twobody dynamics", Phys. Rev. D, 59, 084006, (1999). [gr-qc/9811091]. 6.4, 6.5.3

[97] Buonanno, A., and Damour, T., "Transition from inspiral to plunge in binary black hole coalescences", Phys. Rev. D, 62, 064015, (2000). [gr-qc/0001013]. 6.4, 6.5.2, 6.5.3

[98] Buonanno, A., Kidder, L.E., and Lehner, L., "Estimating the final spin of a binary black hole coalescence", Phys. Rev. D, 77, 026004, (2008). [DOI], [arXiv:0709.3839]. 6.5.2

[99] Buonanno, A., Maggiore, M., and Ungarelli, C., "Spectrum of relic gravitational waves in string cosmology", Phys. Rev. D, 55, 3330-3336, (1997). [gr-qc/9605072]. 8.2.1

[100] Buonanno, A. et al., "Toward faithful templates for non-spinning binary black holes using the effective-one-body approach", Phys. Rev. D, 76, 104049, (2007). [DOI], [arXiv:0706.3732]. 6.5.2, 6.5.2

[101] Burgay, M., D’Amico, N., Possenti, A., Manchester, R.N., Lyne, A.G., Joshi, B.C., McLaughlin, M.A., Kramer, M., Sarkissian, J.M., Camilo, F., Kalogera, V., Kim, C., and Lorimer, D.R., "An increased estimate of the merger rate of double neutron stars from observations of a highly relativistic system", Nature, 426, 531-533, (2003). [astro-ph/0312071]. 3.4.1, 6.3

[102] Caldwell, R.R., Battye, R.A., and Shellard, E.P.S., "Relic Gravitational Waves from Cosmic Strings: Updated Constraints and Opportunities for Detection", Phys. Rev. D, 54, 71467152, (1996). [astro-ph/9607130]. 8.2.1

[103] California Institute of Technology, "LIGO Laboratory Home Page", project homepage. URL (cited on 08 November 2007):

http://www.ligo.caltech.edu. 4.3.1 
[104] Campanelli, M., Lousto, C.O., Marronetti, P., and Zlochower, Y., "Accurate evolutions of orbiting black-hole binaries without excision", Phys. Rev. Lett., 96, 111101, (2006). [DOI], [gr-qc/0511048]. 6.5.2

[105] Campanelli, M., Lousto, C.O., Zlochower, Y., and Merritt, D., "Large Merger Recoils and Spin Flips From Generic Black-Hole Binaries", Astrophys. J., 659, L5-L8, (2007). [grqc/0701164]. 6.5.2, 6.5.2

[106] Capon, R.A., Radiation Reaction Near Black Holes, Ph.D. Thesis, (University of Wales, Cardiff, 1998). Related online version (cited on 26 February 2009):

http://www.aei.mpg.de/pdf/doctoral/RCapon_98.pdf. 6.5.3

[107] Carilli, C., and Rawlings, S., eds., Science with the Square Kilometre Array, New Astron. Rev., vol. 48, (Elsevier, Amsterdam, 2004). Related online version (cited on 17 December 2008):

http://www.skads-eu.org/p/SKA_SciBook.php. 4.4.2, 8

[108] Caroll, S.M., "The Cosmological Constant", Living Rev. Relativity, 4, lrr-2001-1, (2001). URL (cited on 03 September 2007):

http://www.livingreviews.org//rr-2001-1. 8.3

[109] Caron, B. et al. (The VIRGO Collaboration), "The Virgo interferometer", Class. Quantum Grav., 14, 1461-1469, (1997). 1

[110] Caves, C.M., Thorne, K.S., Drever, R.W.P., Sandberg, V.D., and Zimmerman, M., "On the measurement of a weak classical force coupled to a quantum-mechanical oscillator. I. Issues of principle", Rev. Mod. Phys., 52, 341-392, (1980). [DOI]. 3

[111] Chandrasekhar, S., "Solutions of Two Problems in the Theory of Gravitational Radiation", Phys. Rev. Lett., 24, 611-615, (1970). [DOI]. 7.3.4.1

[112] Chandrasekhar, S., The Mathematical Theory of Black Holes, International Series of Monographs on Physics, vol. 69, (Oxford University Press, Oxford; New York, 1992). 6.4

[113] Chatterji, S., Lazzarini, A., Stein, L., Sutton, P.J., Searle, A., and Tinto, M., "Coherent network analysis technique for discriminating gravitational-wave bursts from instrumental noise", Phys. Rev. D, 74, 082005, (2006). [DOI], [gr-qc/0605002]. 5.2

[114] Chernoff, D.F., and Finn, L.S., "Gravitational radiation, inspiraling binaries, and cosmology", Astrophys. J. Lett., 411, L5-L8, (1993). [DOI], [ADS], [gr-qc/9304020]. 5.1.1, 5.3.3, 6, 6.5 .4

[115] Christensen, N., Dupuis, R.J., Woan, G., and Meyer, R., "A Metropolis-Hastings algorithm for extracting periodic gravitational wave signals from laser interferometric detector data", Phys. Rev. D, 70, 022001, (2004). [DOI], [gr-qc/0402038]. 5.1.3.2, 5.3.4

[116] Christensen, N., and Meyer, R., "Markov chain Monte Carlo methods for Bayesian gravitational radiation data analysis", Phys. Rev. D, 58, 082001, (1998). [DOI]. 5.3.4, 6.5.4

[117] Coccia, E., Fafone, V., and Frossati, G., "On the Design of Ultralow Temperature Spherical Gravitational Wave Detectors", in Coccia, E., Pizzella, G., and Ronga, F., eds., Gravitational Wave Experiments, First Edoardo Amaldi Conference, Villa Tuscolana, Frascati, Rome, 14 17 June 1994, pp. 463-478, (World Scientific, Singapore; River Edge, NJ, 1995). 4.1 
[118] Cokelaer, T., "Gravitational waves from inspiralling compact binaries: hexagonal template placement and its efficiency in detecting physical signals", Phys. Rev. D, 76, 102004, (2007). [DOI], [arXiv:0706.4437]. 5.1.3.1

[119] Compton, K.A., and Schutz, B.F., "Bar-Interferometer Observing", in Ciufolini, I., and Fidecaro, F., eds., Gravitational Waves: Sources and Detectors, Proceedings of the International Conference, Cascina, Italy, 19-23 March, 1996, Edoardo Amaldi Foundation, vol. 2, pp. 173-185, (World Scientific, Singapore; River Edge, NJ, 1997). 4.1

[120] Cornish, N.J., and Littenberg, T.B., "Tests of Bayesian Model Selection Techniques for Gravitational Wave Astronomy", Phys. Rev. D, 76, 083006, (2007). [DOI], [arXiv:0704.1808]. $5.3 .4,6.5 .4$

[121] Cornish, N.J., and Porter, E.K., "MCMC Exploration of Supermassive Black Hole Binary Inspirals", Class. Quantum Grav., 23, S761-S768, (2006). [gr-qc/0605085]. 5.3.4, 6.5.4

[122] Cornish, N.J., and Porter, E.K., "Catching supermassive black hole binaries without a net", Phys. Rev. D, 75, 021301, (2007). [DOI], [gr-qc/0605135]. 5.3.4, 6.5.4

[123] Cornish, N.J., and Porter, E.K., "Searching for massive black hole binaries in the first Mock LISA Data Challenge", Class. Quantum Grav., 24, S501-S512, (2007). [DOI], [grqc/0701167]. 6.5.4

[124] Creighton, J.D.E., "Data analysis strategies for the detection of gravitational waves in nonGaussian noise", Phys. Rev. D, 60, 021101, (1999). [gr-qc/9901075]. 4.8

[125] Crowder, J., and Cornish, N.J., "Extracting galactic binary signals from the first round of Mock LISA Data Challenges", Class. Quantum Grav., 24, S575-S586, (2007). [DOI], [arXiv:0704.2917]. 6.5.4

[126] Cruise, A.M., and Ingley, R.M.J., "A prototype gravitational wave detector for $100 \mathrm{MHz",}$ Class. Quantum Grav., 23, 6185-6193, (2006). [DOI]. 4.3.2, 8.2.1

[127] Cutler, C., "Gravitational waves from neutron stars with large toroidal B fields", Phys. Rev. $D, \mathbf{6 6}, 084025,(2002)$. [gr-qc/0206051]. 7.3.6

[128] Cutler, C., and Flanagan, É.É., "Gravitational waves from merging compact binaries: How accurately can one extract the binary's parameters from the inspiral wave form?", Phys. Rev. D, 49, 2658-2697, (1994). [DOI], [gr-qc/9402014]. 5.1.1, 6.5.4

[129] Cutler, C., and Vallisneri, M., "LISA detections of massive black hole inspirals: parameter extraction errors due to inaccurate template waveforms", Phys. Rev. D, 76, 104018, (2007). [DOI], [arXiv:0707.2982]. 6.5.4

[130] Daisuke, T., Ryutaro, T., Koji, A., Noriyasu, N., Kazuhiro, A., Toshitaka, Y., Mitsuhiro, F., Masa-Katsu, F., Akiteru, T., Alessandro, B., Virginio, S., Riccardo, D., Szabolcs, M., Masaki, A., Kimio, T., Tomomi, A., Kazuhiro, Y., Hideki, I., Takashi, U., Shinji, M., Masatake, O., Kazuaki, K., Norichika, A., Nobuyuki, K., Akito, A., Souichi, T., Takayuki, T., Tomiyoshi, H., Akira, Y., Nobuaki, S., Toshitaka, S., and Takakazu, S., "Current status of Japanese detectors", Class. Quantum Grav., 24, S399-S403, (2007). [arXiv:0704.2881]. 4.3.1

[131] Dalal, N., Holz, D.E., Hughes, S.A., and Jain, B., "Short GRB and binary black hole standard sirens as a probe of dark energy", Phys. Rev. D, 74, 063006, (2006). [DOI], [ADS], [astroph/0601275]. 3.4.2, 8.3 
[132] Damour, T., "The problem of motion in Newtonian and Einsteinian gravity", in Hawking, S.W., and Israel, W., eds., Three Hundred Years of Gravitation, pp. 128-198, (Cambridge University Press, Cambridge; New York, 1987). 1.2

[133] Damour, T., Iyer, B.R., and Sathyaprakash, B.S., "Improved filters for gravitational waves from inspiraling compact binaries", Phys. Rev. D, 57, 885-907, (1998). [gr-qc/9708034]. 6.5.3

[134] Damour, T., Iyer, B.R., and Sathyaprakash, B.S., "A comparison of search templates for gravitational waves from binary inspiral", Phys. Rev. D, 63, 044023, (2001). [gr-qc/0010009]. Erratum-ibid. D72 029902 (2005). 6.5.3, 6.5.3.2, 6.5.3.3

[135] Damour, T., and Nagar, A., "Faithful Effective-One-Body waveforms of small-mass-ratio coalescing black-hole binaries", Phys. Rev. D, 76, 064028, (2007). [DOI], [arXiv:0705.2519]. 6.5 .2

[136] Damour, T., and Nagar, A., "Final spin of a coalescing black-hole binary: An effective-onebody approach", Phys. Rev. D, 76, 044003, (2007). [DOI], [arXiv:0704.3550]. 6.5.2

[137] Damour, T., and Nagar, A., "Comparing Effective-One-Body gravitational waveforms to accurate numerical data", Phys. Rev. D, 77, 024043, (2008). [DOI], [arXiv:0711.2628]. 6.5.2

[138] Damour, T., Nagar, A., Dorband, E. N., Pollney, D., and Rezzolla, L., "Faithful EffectiveOne-Body waveforms of equal-mass coalescing black-hole binaries", Phys. Rev. D, 77, 084017, (2008). [DOI], [arXiv:0712.3003]. 6.5.2

[139] Damour, T., Nagar, A., Hannam, M., Husa, S., and Brügmann, B., "Accurate effective-onebody waveforms of inspiralling and coalescing black-hole binaries", Phys. Rev. D, 78, 044039, (2008). [DOI], [arXiv:0803.3162]. 6.5.2

[140] Damour, T., and Vilenkin, A., "Gravitational wave bursts from cosmic strings", Phys. Rev. Lett., 85, 3761-3764, (2000). [DOI], [gr-qc/0004075]. 8.2.1

[141] Damour, T., and Vilenkin, A., "Gravitational wave bursts from cusps and kinks on cosmic strings", Phys. Rev. D, 64, 064008, (2001). [DOI], [gr-qc/0104026]. 8.2.1

[142] Damour, T., and Vilenkin, A., "Gravitational radiation from cosmic (super)strings: Bursts, stochastic background, and observational windows", Phys. Rev. D, 71, 063510, (2005). [DOI], [arXiv:hep-th/0410222]. 6.6.3, 8.2.1

[143] Danzmann, K., Lück, H., Rüdiger, A., Schilling, R., Schrempel, M., Winkler, W., Hough, J., Newton, G.P., Robertson, N.A., Ward, H., Campbell, A.M., Logan, J.E., Robertson, D.I., Strain, K.A., Bennett, J.R.J., Kose, V., Kühne, M., Schutz, B.F., Nicholson, D., Shuttleworth, J., Welling, H., Aufmuth, P., Rinkleff, R., Tünnermann, A., and Willke, B., "GEO 600 - A 600-m Laser Interferometric Gravitational Wave Antenna", in Coccia, E., Pizzella, G., and Ronga, F., eds., Gravitational Wave Experiments, First Edoardo Amaldi Conference, Villa Tuscolana, Frascati, Rome, 14-17 June 1994, pp. 100-111, (World Scientific, Singapore; River Edge, NJ, 1995). 1, 4.3.1

[144] Danzmann, K., and Rüdiger, A., "LISA technology - concept, status, prospects", Class. Quantum Grav., 20, S1-S9, (2003). [DOI]. 4.4.3

[145] Dhurandhar, S.V., and Sathyaprakash, B.S., "Choice of filters for the detection of gravitational waves from coalescing binaries. II. Detection in colored noise", Phys. Rev. D, 49, 1707-1722, (1994). [DOI]. 5.1.3.1 
[146] Dhurandhar, S.V., and Tinto, M., "Astronomical observations with a network of detectors of gravitational waves - I. Mathematical framework and solution of the five detector problem", Mon. Not. R. Astron. Soc., 234, 663, (1988). [ADS]. 4.2.1

[147] Dimmelmeier, H., Font, J.A., and Müller, E., "Relativistic simulations of rotational core collapse. II. Collapse dynamics and gravitational radiation", Astron. Astrophys., 393, 523542, (2002). [astro-ph/0204289v1]. 3.2, 7.4

[148] Dimmelmeier, H., Ott, C.D., Janka, H.-T., Marek, A., and Müller, E., "Generic Gravitational-Wave Signals from the Collapse of Rotating Stellar Cores", Phys. Rev. Lett., 98, 251101, (2007). [DOI], [astro-ph/0702305v2]. 3.2

[149] Drever, R.W.P., "Interferometric detectors for gravitational radiation", in Deruelle, N., and Piran, T., eds., Gravitational Radiation (Rayonnenment Gravitationnel), NATO Advanced Study Institute, Centre de physique des Houches, 2-21 June 1982, pp. 321-338, (NorthHolland; Elsevier, Amsterdam; New York, 1983). 3

[150] Dupuis, R.J., and Woan, G., "Bayesian estimation of pulsar parameters from gravitational wave data", Phys. Rev. D, 72, 102002, (2005). [DOI], [gr-qc/0508096]. 5.1.3.2

[151] Eckart, A., and Genzel, R., "Observations of stellar proper motions near the Galactic Centre", Nature, 383, 415-417, (1996). [DOI]. 7.2 .4

[152] EGO, "European Gravitational Observatory Home Page", project homepage. URL (cited on 08 November 2007):

http://www.ego-gw.it/. 4.3.1

[153] European Space Agency, "Laser Interferometer Space Antenna", project homepage. URL (cited on 08 November 2007):

http://www.esa.int/esaSC/120376_index_0_m.html. 4.4.3

[154] European Space Agency, "Planck Home Page", project homepage. URL (cited on 28 August 2008): http://www.rssd.esa.int/index.php?project=PLANCK. 8.1.4

[155] Faber, J.A., Baumgarte, T.W., Shapiro, S.L., Taniguchi, K., and Rasio, F.A., "Black HoleNeutron Star Binary Merger Calculations: GRB Progenitors and the Stability of Mass Transfer", in Alimi, J.-M., and Füzfa, A., eds., Albert Einstein Century International Conference, Proceedings of the Albert Einstein Century International Conference, 18-22 July 2005, Paris, France, AIP Conference Proceedings, vol. 861, pp. 622-629, (American Institute of Physics, Melville, NY, 2006). [DOI], [astro-ph/0605512]. 7.3.2

[156] Falcke, H.D., van Haarlem, M.P., de Bruyn, A.G., Braun, R., Röttgering, H.J.A., Stappers, B.W., Boland, W.H.W.M., Butcher, H.R., de Geus, E.J., Koopmans, L.V., Fender, R.P., Kuijpers, H.J.M.E., Miley, G.K., Schilizzi, R.T., Vogt, C., Wijers, R.A.M.J., Wise, M.W., Brouw, W.N., Hamaker, J.P., Noordam, J.E., Oosterloo, T., Bähren, L., Brentjens, M.A., Wijnholds, S.J., Bregman, J.D., van Cappellen, W.A., Gunst, A.W., Kant, G.W., Reitsma, J., van der Schaaf, K., and de Vos, C.M., "A very brief description of LOFAR - the Low Frequency Array", in van der Hucht, K.A., ed., Highlights of Astronomy 14, Proceedings of the IAU XXVI General Assembly, 2006, Proceedings of the IAU, vol. 2, pp. 386-387, (Cambridge University Press, Cambridge, 2008). [DOI], [astro-ph/0610652]. 4.4.2

[157] Faulkner, J., "Ultrashort-Period Binaries, Gravitational Radiation, and Mass Transfer. I. The Standard Model, with Applications to WZ Sagittae and Z Camelopardalis", Astrophys. J., 170, L99-L104, (1971). [DOI], [ADS]. 7, 7.1 
[158] Ferrari, V., Matarrese, S., and Schneider, R., "Gravitational Wave Background from a Cosmological Population of Core-Collapse Supernovae", Mon. Not. R. Astron. Soc., 303, 247257, (1999). [astro-ph/9804259]. 8.2.2

[159] Finn, L.S., "Detection, measurement and gravitational radiation", Phys. Rev. D, 46, 52365249, (1992). [gr-qc/9209010]. 5.1.1, 5.3, 5.3.3, 6

[160] Finn, L.S., "Aperture synthesis for gravitational-wave data analysis: Deterministic sources", Phys. Rev. D, 63, 102001, (2001). [gr-qc/0010033]. 4.7.1

[161] Finn, L.S., and Chernoff, D.F., "Observing binary inspiral in gravitational radiation: One interferometer", Phys. Rev. D, 47, 2198-2219, (1993). [gr-qc/9301003]. 5.1.1, 5.3, 5.3.3, 6

[162] Finn, L.S., and Thorne, K.S., "Gravitational waves from a compact star in a circular, inspiral orbit, in the equatorial plane of a massive, spinning black hole, as observed by LISA", Phys. Rev. D, 62, 124021, (2000). [gr-qc/0007074]. 4.5.1

[163] Flanagan, É.É., "Sensitivity of the Laser Interferometer Gravitational Wave Observatory to a stochastic background, and its dependence on the detector orientations", Phys. Rev. D, 48, 2389-2407, (1993). [astro-ph/9305029]. 4.7.3

[164] Flanagan, É.É., and Hughes, S.A., "Measuring gravitational waves from binary black hole coalescences. I. Signal to noise for inspiral, merger and ringdown", Phys. Rev. D, 57, 45354565, (1998). [gr-qc/9701039]. 3.5, 6.4

[165] Flanagan, É.É., and Hughes, S.A., "Measuring gravitational waves from binary black hole coalescences. II. The waves' information and its extraction, with and without templates", Phys. Rev. D, 57, 4566-4587, (1998). [gr-qc/9710129]. 6.5.4

[166] Friedman, J.L., and Schutz, B.F., "Secular instability of rotating newtonian stars", Astrophys. J., 222, 281-296, (1978). [ADS]. 7.3.4.1

[167] Fryer, C.L., and New, K.C.B., "Gravitational Waves from Gravitational Collapse", Living Rev. Relativity, 6, lrr-2003-2, (2003). URL (cited on 03 September 2007): http://www.livingreviews. org/lrr-2003-2. 3.2

[168] Futamase, T., "Point-particle limit and the far-zone quadrupole formula in general relativity", Phys. Rev. D, 32, 2566-2574, (1985). [DOI]. 6.5.3

[169] Futamase, T., and Itoh, Y., "The Post-Newtonian Approximation for Relativistic Compact Binaries", Living Rev. Relativity, 10, lrr-2007-2, (2007). URL (cited on 03 September 2007): http://www.livingreviews.org/lrr-2007-2. 2.4, 6.5.3

[170] Gair, J.R., Barack, L., Creighton, T., Cutler, C., Larson, S.L., Phinney, E.S., and Vallisneri, M., "Event rate estimates for LISA extreme mass ratio capture sources", Class. Quantum Grav., 21, S1595-S1606, (2004). [gr-qc/0405137]. 6.6.2

[171] Gair, J.R., and Glampedakis, K., "Improved approximate inspirals of test-bodies into Kerr black holes", Phys. Rev. D, 73, 064037, (2006). [gr-qc/0510129]. 3.4.6

[172] Gair, J.R, and Jones, G., "Detecting extreme mass ratio inspiral events in LISA data using the hierarchical algorithm for clusters and ridges (HACR)", Class. Quantum Grav., 27, 1145-1168, (2007). [DOI], [gr-qc/0610046]. 5.2 
[173] Gavriil, F.P., Gonzalez, M.E., Gotthelf, E.V., Kaspi, V.M., Livingstone, M.A., and Woods, P.M., "Magnetar-Like Emission from the Young Pulsar in Kes 75", Science, 319, 1802-1805, (2008). [DOI], [arXiv:0802.1704]. 7.3.3

[174] Gebhardt, K., Bender, R., Bower, G., Dressler, A., Faber, S.M., Filippenko, A.V., Green, R., Grillmair, C., Ho, L.C., Kormendy, J., Lauer, T.R., Magorrian, J., Pinkney, J., Richstone, D., and Tremaine, S., "A Relationship between Nuclear Black Hole Mass and Galaxy Velocity Dispersion", Astrophys. J. Lett., 539, L13-L16, (2000). [astro-ph/0006289]. 7.2.4

[175] Giazotto, A. et al., "The VIRGO Experiment: Status of the Art", in Coccia, E., Pizzella, G., and Ronga, F., eds., Gravitational Wave Experiments, First Edoardo Amaldi Conference, Villa Tuscolana, Frascati, Rome, $14-17$ June 1994, pp. 86-99, (World Scientific, Singapore; River Edge, NJ, 1995). 1, 4.3.1

[176] Glampedakis, K., "Extreme Mass Ratio Inspirals: LISA's unique probe of black hole gravity", Class. Quantum Grav., 22, S605-S659, (2005). [gr-qc/0509024]. 3.4.6

[177] Glampedakis, K., and Babak, S., "Mapping spacetimes with LISA: Inspiral of a test-body in a 'quasi-Kerr' field", Class. Quantum Grav., 23, 4167-4188, (2006). [gr-qc/0510057]. 3.4.6, 6.6

[178] Glampedakis, K., Hughes, S.A., and Kennefick, D., "Approximating the inspiral of test bodies into Kerr black holes", Phys. Rev. D, 66, 064005, (2002). [gr-qc/0205033]. 3.4.6

[179] Glampedakis, K., and Kennefick, D., "Zoom and whirl: Eccentric equatorial orbits around spinning black holes and their evolution under gravitational radiation reaction", Phys. Rev. D, 66, 044002, (2002). [gr-qc/0203086]. 3.4.6

[180] Gonzalez, J.A., Sperhake, U., Brügmann, B., Hannam, M., and Husa, S., "Total recoil: the maximum kick from nonspinning black-hole binary inspiral", Phys. Rev. Lett., 98, 091101, (2007). [DOI], [gr-qc/0610154]. 6.5.2

[181] Gottardi, L., de Waard, A., Usenko, A., Frossati, G., Podt, M., Flokstra, J., Bassan, M., Fafone, V., Minenkov, Y., and Rocchi, A., "Sensitivity of the spherical gravitational wave detector MiniGRAIL operating at 5 K", Phys. Rev. D, 76, 102005, (2007). [arXiv:0705.0122]. 4.1

[182] Grishchuk, L.P., "Amplification of gravitational waves in an istropic universe", Sov. Phys. JETP, 40, 409-415, (1975). 8.2.1

[183] Grishchuk, L.P., "The implications of the microwave background anisotropies for laserinterferometer-tested gravitational waves", Class. Quantum Grav., 14, 1445-1454, (1997). [DOI], [gr-qc/9609062]. 8.2.1

[184] Gürsel, Y., and Tinto, M., "Near optimal solution to the inverse problem for gravitationalwave bursts", Phys. Rev. D, 40, 3884-3938, (1989). 4.7.2

[185] Haehnelt, M.G., "Supermassive black holes as sources for LISA", in Folkner, W.M., ed., Laser Interferometer Space Antenna (LISA), The Second International LISA Symposium on the Detection and Observation of Gravitational Waves in Space, Pasadena, California, July 1998, AIP Conference Proceedings, vol. 456, pp. 45-49, (American Institute of Physics, Woodbury, NY, 1998). [DOI]. 3.4.5

[186] Helstrom, C.W., Statistical Theory of Signal Detection, International Series of Monographs in Electronics and Instrumentation, vol. 9, (Pergamon Press, Oxford; New York, 1968), 2nd edition. 5.1, 5.3, 5.3.1, 5.3.2 
[187] Heng, I.S., Balasubramanian, R., Sathyaprakash, B.S., and Schutz, B.F., "First steps towards characterizing the hierarchical algorithm for curves and ridges pipeline", Class. Quantum Grav., 21, S821-S826, (2004). [DOI]. 5.2

[188] Herrmann, F., Hinder, I., Shoemaker, D., Laguna, P., and Matzner, R.A., "Gravitational recoil from spinning binary black hole mergers", Astrophys. J., 661, 430-436, (2007). [DOI], [gr-qc/0701143]. 6.5.2

[189] Hewish, A., Bell, S.J., Pilkington, J.D.H., Scott, P.F., and Collins, R.A., "Observation of a Rapidly Pulsating Radio Source", Nature, 217, 709-713, (1968). [DOI]. 1

[190] Hils, D., Bender, P.L., and Webbink, R.F., "Gravitational radiation from the Galaxy", Astrophys. J., 360, 75-94, (1990). [DOI]. 8.2.2

[191] Hjorth, J., Sollerman, J., Moller, P., Fynbo, J.P.U., Woosley, S.E., Kouveliotou, C., Tanvir, N.R., Greiner, J., Andersen, M.I., Castro-Tirado, A.J., Castro Cerón, J.M., Fruchter, A.S., Gorosabel, J., Jakobsson, P., Kaper, L., Klose, S., Masetti, N., Pedersen, H., Pedersen, K., Pian, E., Palazzi, E., Rhoads, J.E., Rol, E., van den Heuvel, E.P.J., Vreeswijk, P.M., Watson, D., and Wijers, R.A.M.J., "A very energetic supernova associated with the $\gamma$-ray burst of 29 March 2003", Nature, 423, 847-850, (2003). [DOI], [astro-ph/0306347]. 3.2

[192] Hogan, C.J., "Cosmological Gravitational Wave Backgrounds", in Folkner, W.M., ed., Laser Interferometer Space Antenna (LISA), The Second International LISA Symposium on the Detection and Observation of Gravitational Waves in Space, Pasadena, California, July 1998, AIP Conference Proceedings, vol. 456, pp. 79-86, (American Institute of Physics, Woodbury, NY, 1998). [DOI], [astro-ph/9809364]. 8.2.1

[193] Hogan, C.J., "Measurement of quantum fluctuations in geometry", Phys. Rev. D, 77, 104031, (2008). [DOI], [arXiv:0712.3419]. 6.6.3

[194] Hogan, C.J., and Bender, P.L., "Estimating stochastic gravitational wave backgrounds with the Sagnac calibration", Phys. Rev. D, 64, 062002, (2001). [astro-ph/0104266]. 8.1.2

[195] Holz, D.E., and Hughes, S.A., "Using gravitational-wave standard sirens", Astrophys. J., 629, 15-22, (2005). [astro-ph/0504616]. 3.4.2, 6.5.1, 8.3

[196] Hough, J., "LISA - Laser Interferometer Space Antenna for Gravitational Wave Measurements", in Coccia, E., Pizzella, G., and Ronga, F., eds., Gravitational Wave Experiments, First Edoardo Amaldi Conference, Villa Tuscolana, Frascati, Rome, 14-17 June 1994, pp. 50-63, (World Scientific, Singapore; River Edge, NJ, 1995). 4.4.3

[197] Hough, J., and Rowan, S., "Gravitational Wave Detection by Interferometry (Ground and Space)", Living Rev. Relativity, 3, lrr-2000-3, (2000). URL (cited on 03 September 2007): http://www.livingreviews.org/lrr-2000-3. 1, 4.2.1

[198] Hughes, S.A., "Gravitational waves from extreme mass ratio inspirals: Challenges in mapping the spacetime of massive, compact objects", Class. Quantum Grav., 18, 4067-4074, (2001). [gr-qc/0008058]. 3.4.6

[199] Hughes, S.A., and Blandford, R.D., "Black hole mass and spin coevolution by mergers", Astrophys. J. Lett., 585, L101-L104, (2003). [astro-ph/0208484]. 6.5.2

[200] Hulse, R.A., "Nobel Lecture: The discovery of the binary pulsar", Rev. Mod. Phys., 66, 699-710, (1994). [DOI]. Related online version (cited on 26 February 2009):

http://nobelprize.org/nobel_prizes/physics/laureates/1993/hulse-lecture.html. 6.3 
[201] Hulse, R.A., and Taylor, J.H., "Discovery of a pulsar in a binary system", Astrophys. J., 195, L51-L53, (1975). [ADS]. 7.3.6

[202] INFN, "IGEC: International Gravitational Event Collaboration", project homepage. URL (cited on 08 November 2007):

http://igec.lnl.infn.it/. 4.1

[203] INPE, Brasil, "Gravitational Waves - INPE", project homepage. URL (cited on 08 November 2007):

http://www.das.inpe.br/graviton/english.html. 4.1

[204] Jackson, N., "The Hubble Constant", Living Rev. Relativity, 10, lrr-2007-4, (2007). URL (cited on 01 September 2008):

http://www. livingreviews .org/lrr-2007-4. 8.3

[205] Jaranowski, P., and Królak, A., "Optimal solution to the inverse problem for the gravitational wave signal of a coalescing compact binary", Phys. Rev. D, 49, 1723-1739, (1994). [DOI]. 4.7.1

[206] Jaranowski, P., and Królak, A., "Gravitational-Wave Data Analysis. Formalism and Sample Applications: The Gaussian Case", Living Rev. Relativity, 8, lrr-2005-3, (2005). URL (cited on 03 September 2007):

http://www. livingreviews.org/lrr-2005-3. 5

[207] Jaranowski, P., Królak, A., Kokkotas, K. D., and Tsegas, G., "On the estimation of parameters of the gravitational-wave signal from a coalescing binary by a network of detectors", Class. Quantum Grav., 13, 1279-1307, (1996). [DOI]. 4.7.1

[208] Jenet, F.A., Hobbs, G.B., van Straten, W., Manchester, R.N., Bailes, M., Verbiest, J.P.W., Edwards, R.T., Hotan, A.W., Sarkissian, J.M., and Ord, S.M., "Upper bounds on the low-frequency stochastic gravitational wave background from pulsar timing observations: Current limits and future prospects", Astrophys. J., 653, 1571-1576, (2006). [DOI], [astroph/0609013]. 4.4.2

[209] Jenet, F.A., Lommen, A., Larson, S.L., and Wen, L., "Constraining the Properties of Supermassive Black Hole Systems Using Pulsar Timing: Application to 3C 66B", Astrophys. J., 606, 799-803, (2004). [astro-ph/0310276]. 4.4.2

[210] Kalogera, V., Kim, C., and Lorimer, D.R., "The Strongly Relativistic Double Pulsar and LISA (Galactic Double Neutron Stars for LISA)", Invited talk at the 5th International LISA Symposium, ESTEC, Noordwijk, The Netherlands, 12 - 15 July 2004, conference paper, (2004). Related online version (cited on 17 December 2008):

http://www . astro.northwestern.edu/Vicky/TALKS/LISA_0737.ppt. 7.3.6

[211] Kalogera, V., Kim, C., Lorimer, D.R., Burgay, M., D’Amico, N., Possenti, A., Manchester, R.N., Lyne, A.G., Joshi, B.C., McLaughlin, M.A., Kramer, M., Sarkissian, J.M., and Camilo, F., "The Cosmic Coalescence Rates for Double Neutron Star Binaries", Astrophys. J. Lett., 601, L179-L182, (2004). [DOI], [astro-ph/0312101]. 3.4.1

[212] Kaspi, V.M., Taylor, J.H., and Ryba, M.F., "High-precision timing of millisecond pulsars. III. Long-term monitoring of PSRs B1855+09 and B1937+21", Astrophys. J., 428, 713-728, (1994). [ADS]. 8.1.3 
[213] Kawamura, S., Nakamura, T., Ando, M., Seto, N., and Tsubono, K. et al., "The Japanese space gravitational wave antenna-DECIGO", Class. Quantum Grav., 23, S125-S131, (2006). [DOI]. 4.4.3

[214] Keating, B.G., "An 'Ultrasonic Image' of the Embryonic Universe: CMB Polarization Tests of the Inflationary Paradigm", arXiv e-print, (2008). [arXiv:0806.1781]. 8, 8.1.4

[215] Keating, B.G., Polnarev, A.G., Miller, N.J., and Baskaran, D., "The Polarization of the Cosmic Microwave Background Due to Primordial Gravitational Waves", Int. J. Mod. Phys. A, 21, 2459-2479, (2006). [astro-ph/0607208]. 8.1.4

[216] Klebesadel, R.W., Strong, I.B., and Olson, R.A., "Observations of Gamma-Ray Bursts of Cosmic Origin", Astrophys. J., 182, L85-L88, (1973). 1

[217] Klimenko, S., and Mitselmakher, G., "A wavelet method for detection of gravitational wave bursts", Class. Quantum Grav., 21, S1819-S1830, (2004). [DOI]. 5.2

[218] Klimenko, S., Yakushin, I., Mercer, A., and Mitselmakher, G., "Coherent method for detection of gravitational wave bursts", Class. Quantum Grav., 25, 114029, (2008). [DOI], [arXiv:0802.3232]. 5.2

[219] Knispel, B., and Allen, B., "Blandford's Argument: The Strongest Continuous Gravitational Wave Signal", Phys. Rev. D, 78, 044031, (2008). [arXiv:0804.3075]. 7.3.6

[220] Kokkotas, K.D., and Schmidt, B., "Quasi-Normal Modes of Stars and Black Holes", Living Rev. Relativity, 2, lrr-1999-2, (1999). URL (cited on 03 September 2007):

http://www.livingreviews.org/lrr-1999-2. 3.5

[221] Komossa, S., Burwitz, V., Hasinger, G., Predehl, P., Kaastra, J.S., and Ikebe, Y., "Discovery of a Binary Active Galactic Nucleus in the Ultraluminous Infrared Galaxy NGC 6240 Using Chandra", Astrophys. J. Lett., 582, L15-L19, (2003). [astro-ph/0212099]. 3.4 .5

[222] Komossa, S., Zhou, H., and Lu, H., "A recoiling supermassive black hole in the quasar SDSSJ092712.65+294344.0?", Astrophys. J. Lett., 678, L81-L84, (2008). [DOI], [arXiv:0804.4585]. 3.4.5, 6.5.2, 7.2.4

[223] Kramer, M., "Pulsars with the SKA", in Kramer, M., and Rawlings, S., eds., The Scientific Promise of the SKA, Proceedings of a workshop held at Oxford, 7 November 2002, pp. 85-92, (2003). [astro-ph/0306456]. 8.1.3

[224] Kramer, M., "Fundamental Physics with the SKA: Strong-Field Tests of Gravity Using Pulsars and Black Holes", in Lobanov, A.P., Zensus, J.A., Cesarsyk, C., and Diamond, P., eds., Exploring the Cosmic Frontier: Astrophysical Instruments for the 21st Century, Proceedings of the conference held in Berlin, Germany, 18-21 May 2004, ESO Astrophysics Symposia, pp. 87-90, (Springer, Berlin; New York, 2006). [DOI], [astro-ph/0409020]. 8

[225] Krishnan, B., Sintes, A.M., Papa, M.A., Schutz, B.F., Frasca, S., and Palomba, C., "The Hough transform search for continuous gravitational waves", Phys. Rev. D, 70, 082001, (2004). [DOI], [gr-qc/0407001]. 5.1.3.2

[226] Królak, A., and Schutz, B.F., "Coalescing binaries - Probe of the universe", Gen. Relativ. Gravit., 19, 1163-1171, (1987). [DOI]. 8.3

[227] Laboratori Nationali Legnaro, "AURIGA Bar Detector", project homepage. URL (cited on 08 November 2007):

http://www.auriga.lnl.infn.it/. 1, 4.1 
[228] Lackey, B.D., Nayyar, M., and Owen, B.J., "Observational constraints on hyperons in neutron stars", Phys. Rev. D, 73, 024021, (2006). [astro-ph/0507312]. 7.3.4.2

[229] Lahav, O., and Suto, Y., "Measuring our Universe from Galaxy Redshift Surveys", Living Rev. Relativity, 7, lrr-2004-8, (2004). URL (cited on 07 December 2004): http://www. livingreviews.org/lrr-2004-8. 8.2.1

[230] Landgraf, M., Hechler, M., and Kemble, S., "Mission design for LISA Pathfinder", Class. Quantum Grav., 22, S487-S492, (2005). [gr-qc/0411071]. 4.4.3

[231] Lang, R.N., and Hughes, S.A., "Measuring coalescing massive binary black holes with gravitational waves: The impact of spin-induced precession", Phys. Rev. D, 74, 122001, (2006). [DOI], [gr-qc/0608062]. 13

[232] Lang, R.N., and Hughes, S.A., "Localizing coalescing massive black hole binaries with gravitational waves", Astrophys. J., 677, 1184-1200, (2008). [arXiv:0710.3795]. 6.5.4.2, 8.3

[233] Lattimer, J.M., and Swesty, F.D., "A generalized equation of state for hot, dense matter", Nucl. Phys. A, 535, 331-376, (1991). [DOI]. 7.3 .2

[234] Leiden University, "MiniGRAIL", project homepage. URL (cited on 08 November 2007): http://www.minigrail.nl/. 4.1

[235] LIGO Laboratory, "Advanced LIGO", project homepage. URL (cited on 08 November 2007): http://www.ligo.caltech.edu/advLIGO/scripts/summary.shtml. 4.3.1

[236] LIGO Laboratory, "LIGO Scientific Collaboration", project homepage. URL (cited on 08 November 2007): http://ligo.org/. 4.3 .1

[237] LIGO Scientific Collaboration, "LSC Publications", online resource. URL (cited on 08 November 2007):

http://www.lsc-group.phys.uwm.edu/ppcomm/Papers.html. 4.3.1

[238] Lindblom, L., and Detweiler, S.L., "On the secular instabilities of the Maclaurin spheroids", Astrophys. J., 211, 565-567, (1977). [ADS]. 7.3.4.1

[239] Lindblom, L., and Mendell, G., "Does gravitational radiation limit the angular velocities of superfluid neutron stars?", Astrophys. J., 444, 804-809, (1995). [DOI], [ADS]. 7.3.4.1

[240] Lindblom, L., and Owen, B.J., "Effect of hyperon bulk viscosity on neutron-star r-modes", Phys. Rev. D, 65, 063006, (2002). [astro-ph/0110558]. 7.3.4.2

[241] Lindblom, L., Owen, B.J., and Morsink, S.M., "Gravitational radiation instability in hot young neutron stars", Phys. Rev. Lett., 80, 4843-4846, (1998). [DOI], [gr-qc/9803053]. 7.3.4.2

[242] Lorimer, D.R., "Binary and Millisecond Pulsars", Living Rev. Relativity, 8, lrr-2005-7, (2005). URL (cited on 03 September 2007):

http://www.livingreviews.org/lrr-2005-7. 3.4.1, 4.4.2, 6.3

[243] Louisiana State University, "ALLEGRO Bar Detector", project homepage. URL (cited on 08 November 2007): http://gravity.phys.1su.edu/. 4.1

[244] Lück, H., "The GEO600 project", Class. Quantum Grav., 14, 1471-1476, (1997). [DOI]. 1 
[245] Lück, H. et al., "Status of the GEO600 detector", Class. Quantum Grav., 23, S71-S78, (2006). [DOI]. 1

[246] Lyne, A.G., Burgay, M., Kramer, M., Possenti, A., Manchester, R.N., Camilo, F., McLaughlin, M.A., Lorimer, D.R., D'Amico, N., Joshi, B.C., Reynolds, J., and Freire, P.C.C., "A Double-Pulsar System: A Rare Laboratory for Relativistic Gravity and Plasma Physics", Science, 303, 1153-1157, (2004). [DOI], [astro-ph/0401086]. 3.4.3, 7.3.6

[247] Lyne, A.G., Burgay, M., Kramer, M., Possenti, A., Manchester, R.N., Camilo, F., McLaughlin, M.A., Lorimer, D.R., D'Amico, N., Joshi, B.C., Reynolds, J., and Freire, P.C.C., "A Double-Pulsar System: A Rare Laboratory for Relativistic Gravity and Plasma Physics", Science, 303, 1153-1157, (2004). [astro-ph/0401086]. 6.3

[248] Maartens, R., "Brane-World Gravity", Living Rev. Relativity, 7, lrr-2004-7, (2004). URL (cited on 07 December 2004):

http://www.livingreviews.org/lrr-2004-7. 8.2.1

[249] MacFadyen, A.I., and Woosley, S.E., "Collapsars: Gamma-ray bursts and explosions in 'failed supernovae", Astrophys. J., 524, 262-289, (1999). [DOI], [astro-ph/9810274]. 3.2

[250] MacLeod, C.L., and Hogan, C.J., "Precision of Hubble constant derived using black hole binary absolute distances and statistical redshift information", Phys. Rev. D, 77, 043512, (2008). [DOI], [arXiv:0712.0618]. 8.3

[251] Marronetti, P., Tichy, W., Brügmann, B., Gonzalez, J., and Sperhake, U., "High-spin binary black hole mergers", Phys. Rev. D, 77, 064010, (2008). [DOI], [arXiv:0709.2160]. 6.5.2

[252] McClelland, D.E., and Bachor, H.-A., eds., Gravitational Astronomy: Instrument Design and Astrophysical Prospects, Proceedings of the Elizabeth and Frederick White Research Conference, Canberra, Australia, September 24-26, 1990, (World Scientific, Singapore; River Edge, NJ, 1991). 4.3.1

[253] Megevand, A., and Astorga, F., "Generation of baryon inhomogeneities in the electroweak phase transition", Phys. Rev. D, 71, 023502, (2005). [hep-ph/0409321]. 8.2.1

[254] Merritt, D., and Ekers, R.D., "Tracing black hole mergers through radio lobe morphology", Science, 297, 1310-1313, (2002). [DOI], [astro-ph/0208001]. 3.4.5

[255] Merritt, D., and Milosavljević, M., "Massive Black Hole Binary Evolution", Living Rev. Relativity, 8, lrr-2005-8, (2005). URL (cited on 03 September 2007):

http://www. livingreviews . org/lrr-2005-8. 7.2.4

[256] Mészáros, P., and Rees, M.J., "Relativistic fireballs and their impact on external matter - Models for cosmological gamma-ray bursts", Astrophys. J., 405, 278-284, (1993). [DOI], [ADS]. 6.1

[257] Milosavljević, M., and Phinney, E.S., "The Afterglow of Massive Black Hole Coalescence", Astrophys. J. Lett., 622, L93-L96, (2005). [astro-ph/0410343]. 7.4, 8.3

[258] Mino, Y., Shibata, M., and Tanaka, T., "Gravitational waves induced by a spinning particle falling into a rotating black hole", Phys. Rev. D, 53, 622-634, (1996). [DOI]. 6.5.3

[259] Misner, C.W., Thorne, K.S., and Wheeler, J.A., Gravitation, (W.H. Freeman, San Francisco, 1973). $1.2,2.6,2.6,6.2$ 
[260] Mohanty, S.D., "A robust test for detecting non-stationarity in data from gravitational wave detectors", Phys. Rev. D, 61, 122002, (2000). [gr-qc/9910027]. 4.8

[261] MPI for Gravitational Physics (Albert Einstein Institute), "GEO600: The German-British Gravitational Wave Detector", project homepage. URL (cited on 08 November 2007): http://geo600.aei.mpg.de. 4.3.1

[262] Mukhanov, V.F., Feldman, H.A., and Brandenberger, R.H., "Theory of cosmological perturbations", Phys. Rep., 215, 203-333, (1992). [DOI]. 8.2.1

[263] Mukhopadhyay, H., Sago, N., Tagoshi, H., Dhurandhar, S., Takahashi, H., and Kanda, N., "Detecting gravitational waves from inspiraling binaries with a network of detectors: coherent versus coincident strategies", Phys. Rev. D, 74, 083005, (2006). [gr-qc/0608103]. 4.7.1

[264] Müller, E., "Gravitational Waves from Core Collapse Supernovae", in Marck, J.-A., and Lasota, J.-P., eds., Relativistic Gravitation and Gravitational Radiation, Proceedings of the Les Houches School of Physics, held in Les Houches, Haute Savoie, 26 September-6 October, 1995, Cambridge Contemporary Astrophysics, pp. 273-308, (Cambridge University Press, Cambridge, 1997). 3.2

[265] Nakamura, T., Sasaki, M., Tanaka, T., and Thorne, K.S., "Gravitational waves from coalescing black hole MACHO binaries", Astrophys. J. Lett., 487, L139-L142, (1997). [astroph/9708060]. 7.2 .2

[266] NASA, "Laser Interferometer Space Antenna", project homepage. URL (cited on 08 November 2007):

http://lisa.nasa.gov/. 4.4.3

[267] NASA, "NASA Vision Missions", project homepage. URL (cited on 08 November 2007): http://universe.nasa.gov/program/vision.html. 4.4.3

[268] National Astronomical Observatory, Japan, "Large-Scale Cryogenic Gravitational-Wave Telescope Project", project homepage. URL (cited on 28 August 2008): http://www.icrr.u-tokyo.ac.jp/gr/LCGT.html. 4.3.1

[269] National Astronomical Observatory, Japan, "TAMA300 Project", project homepage. URL (cited on 08 November 2007):

http://tamago.mtk.nao.ac.jp/. 4.3.1

[270] Nayyar, M., and Owen, B.J., "R-modes of accreting hyperon stars as persistent sources of gravitational waves", Phys. Rev. D, 73, 084001, (2006). [DOI], [astro-ph/0512041]. 3.3

[271] Nelemans, G., "AM CVn stars", in Hameury, J.-M., and Lasota, J.-P., eds., The Astrophysics of Cataclysmic Variables and Related Objects, Proceedings of a meeting held in Strasbourg, France, 11-16 July 2004, ASP Conference Series, vol. 330, pp. 27-40, (Astronomical Society of the Pacific, San Francisco, 2005). [ADS], [astro-ph/0409676]. 7.4

[272] Nelemans, G., Yungelson, L.R., and Portegies Zwart, S.F., "The gravitational wave signal from the Galactic disk population of binaries containing two compact objects", Astron. Astrophys., 375, 890-898, (2001). [DOI], [astro-ph/0105221]. 4.5.1

[273] Nicholson, D., Dickson, C.A., Watkins, W.J., Schutz, B.F., Shuttleworth, J., Jones, G.S., Robertson, D.I., MacKenzie, N.L., Strain, K.A., Meers, B.J., Newton, G.P., Ward, H., Cantley, C.A., Robertson, N.A., Hough, J., Danzmann, K., Niebauer, T.M., Ruediger, A., Schilling, R., Schnupp, L., and Winkler, W., "Results of the first coincident observations by 
two laser-interferometric gravitational wave detectors", Phys. Lett. A, 218, 175-180, (1996). [gr-qc/9605048]. 5

[274] Nicholson, D., and Vecchio, A., "Bayesian bounds on parameter estimation accuracy for compact coalescing binary gravitational wave signals", Phys. Rev. D, 57, 4588-4599, (1998). [DOI], [gr-qc/9705064]. 5.3

[275] Noyola, E., Gebhardt, K., and Bergmann, M., "Gemini and Hubble Space Telescope Evidence for an Intermediate Mass Black Hole in $\omega$ Centauri", Astrophys. J., 676, 1008-1015, (2008). [DOI], [arXiv:0801.2782]. 7.2.3

[276] Ott, C.D., Burrows, A., Dessart, L., and Livne, E., "A New Mechanism for Gravitational Wave Emission in Core-Collapse Supernovae", Phys. Rev. Lett., 96, 201102, (2006). [astroph/0605493v1]. 3.2

[277] Owen, B.J., "Search templates for gravitational waves from inspiralling binaries: Choise of template spacing", Phys. Rev. D, 53, 6749-6761, (1996). [gr-qc/9511032]. 5.1.3.1, 5.3.1, 5.3.2, 6

[278] Owen, B.J., Lindblom, L., Cutler, C., Schutz, B.F., Vecchio, A., and Andersson, N., "Gravitational waves from hot young rapidly rotating neutron stars", Phys. Rev. D, 58, 084020, (1998). [gr-qc/9804044]. 7.3.1, 7.3.4.2

[279] Owen, B.J., and Sathyaprakash, B.S., "Matched filtering of gravitational waves from inspiraling compact binaries: Computational cost and template placement", Phys. Rev. D, 60, 022002, (1999). [DOI], [gr-qc/9808076]. 5.1.3.1, 5.3.2

[280] Page, L., Hinshaw, G., Komatsu, E., Nolta, M.R., Spergel, D.N., Bennett, C.L., Barnes, C., Bean, R., Dore, O., Dunkley, J., Halpern, M., Hill, R. S., Jarosik, N., Kogut, A., Limon, M., Meyer, S.S., Odegard, N., Peiris, H.V., Tucker, G.S., Verde, L., Weiland, J.L., Wollack, E., and Wright, E.L., "Three Year Wilkinson Microwave Anisotropy Probe (WMAP) Observations: Polarization Analysis", Astrophys. J. Suppl. Ser., 170, 335-376, (2007). [DOI], [astro-ph/0603450]. 8.1.4

[281] Pagel, B.E.J., "Helium and Big Bang nucleosynthesis", Phys. Rep., 333, 433-447, (2000). [DOI]. 3.6

[282] Pai, A., Dhurandhar, S., and Bose, S., "A data-analysis strategy for detecting gravitationalwave signals from inspiraling compact binaries with a network of laser-interferometric detectors", Phys. Rev. D, 64, 042004, (2001). [gr-qc/0009078]. 4.7.1

[283] Pan, Y., Buonanno, A., Chen, Y., and Vallisneri, M., "Physical template family for gravitational waves from precessing binaries of spinning compact objects: Application to single-spin binaries", Phys. Rev. D, 69, 104017, (2004). [gr-qc/0310034]. Erratum-ibid. D74, 029905(E) (2006). 5.3.2

[284] Pan, Y. et al., "A data-analysis driven comparison of analytic and numerical coalescing binary waveforms: Nonspinning case", Phys. Rev. D, 77, 024014, (2008). [DOI], [arXiv:0704.1964]. 6.5 .2

[285] Payne, D.J.B., Melatos, A., and Phinney, E.S., "Gravitational waves from an accreting neutron star with a magnetic mountain", in Centrella, J.M., ed., Astrophysics of Gravitational Wave Sources, College Park, Maryland, April 24-26, 2003, AIP Conference Proceedings, vol. 686, pp. 92-95, (American Institute of Physics, Melville, NY, 2003). [DOI]. 3.3 
[286] Pearce, F.R., Jenkins, A., Frenk, C.S., White, S.D.M., Thomas, P.A., Couchman, H.M.P., Peacock, J.A., and Efstathiou, G., "Simulations of galaxy formation in a cosmological volume", Mon. Not. R. Astron. Soc., 326, 649, (2001). [astro-ph/0010587]. 8.2.1

[287] Penzias, A.A., and Wilson, R.W., "A Measurement of Excess Antenna Temperature at 4080 Mc/s", Astrophys. J., 142, 419-421, (1965). [ADS]. 1

[288] Perlmutter, S. et al. (The Supernova Cosmology Project), "Measurements of $\Omega$ and $\Lambda$ from 42 High-Redshift Supernovae", Astrophys. J., 517, 565-586, (1999). [DOI], [astro-ph/9812133]. 1

[289] Perryman, M.A.C., Turon, C., and O'Flaherty, K.S., eds., The Three-Dimensional Universe with Gaia, Proceedings of the Symposium held at the Observatoire de Paris-Meudon, 4-7 October 2004, ESA Conference Proceedings, vol. SP-576, (ESA Publications Division, Noordwijk, 2005). Related online version (cited on 05 September 2008): http: //www.rssd.esa.int/index.php?project=Gaia\&page=Gaia_2004_Proceedings. 7.1 .1

[290] Peters, P.C., and Mathews, J., "Gravitational radiation from point masses in a Keplerian orbit", Phys. Rev., 131, 435-440, (1963). [DOI]. 3.4.2, 3.4.3, 6.3

[291] Plissi, M.V., Strain, K.A., Torrie, C.I., Robertson, N.A., Killbourn, S., Rowan, S., Twyford, S., Ward, H., Skeldon, K.D., and Hough, J., "Aspects of the suspension system for GEO600", Rev. Sci. Instrum., 69, 3055-3061, (1998). [DOI]. 1

[292] Poisson, E., "The Motion of Point Particles in Curved Spacetime", Living Rev. Relativity, 7, lrr-2004-6, (2004). URL (cited on 03 September 2007):

http://www. livingreviews.org/lrr-2004-6. 6.5.3, 6.6.2

[293] Poisson, E., and Will, C.M., "Gravitational waves from inspiraling compact binaries: Parameter estimation using second-post-Newtonian wave forms", Phys. Rev. D, 52, 848-855, (1995). [DOI], [gr-qc/9502040]. 6.5.4

[294] Pollney, D., Reisswig, C., Rezzolla, L., Szilágyi, B., Ansorg, M., Deris, B., Diener, P., Dorband, E.N., Koppitz, M., Nagar, A., and Schnetter, E., "Recoil velocities from equal-mass binary black-hole mergers: a systematic investigation of spin-orbit aligned configurations", Phys. Rev. D, 76, 124002, (2007). [DOI], [arXiv:0707.2559]. 6.5.2, 6.5.2

[295] Portegies Zwart, S.F., and McMillan, S.L.W., "Black hole mergers in the universe", Astrophys. J. Lett., 528, L17-L20, (2000). [gr-qc/9910061]. 7.2.2

[296] Press, W.H., "Long Wave Trains of Gravitational Waves from a Vibrating Black Hole", Astrophys. J. Lett., 170, L105-L108, (1971). [ADS]. 3.5, 6.4

[297] Press, W.H., and Teukolsky, S.A., "Perturbations of a Rotating Black Hole. II. Dynamical Stability of the Kerr Metric", Astrophys. J., 185, 649-673, (1973). [ADS]. 6.4

[298] Pretorius, F., "Evolution of binary black-hole spacetimes", Phys. Rev. Lett., 95, 121101, (2005). [DOI], [gr-qc/0507014]. 6.4, 6.5.2

[299] Pretorius, F., "Binary Black Hole Coalescence", in Colpi, M., Casella, P., Gorini, V., Moschella, U., and Possenti, A., eds., Physics of Relativistic Objects in Compact Binaries: From Birth to Coalescence, Astrophysics and Space Science Library, vol. 359, (Springer, Berlin; New York, 2009). [arXiv:0710.1338]. 6.5.2, 6.5.2 
[300] Pryke, C., Ade, P., Bock, J., Bowden, M., Brown, M.L., Cahill, G., Castro, P.G., Church, S., Culverhouse, T., Friedman, R., Ganga, K., Gear, W.K., Gupta, S., Hinderks, J., Kovac, J., Lange, A.E., Leitch, E., Melhuish, S.J., Memari, Y., Murphy, J.A., Orlando, A., Schwarz, R., O'Sullivan, C., Piccirillo, L., Rajguru, N., Rusholme, B., Taylor, A.N., Thompson, K.L., Turner, A.H., Wu, E.Y.S., and Zemcov, M. (QUaD collboration), "Second and third season QUaD CMB temperature and polarization power spectra", Astrophys. J., submitted, (2008). [arXiv:0805.1944]. 8, 8.1.4

[301] Quinn, T.C., and Wald, R.M., "An axiomatic approach to electromagnetic and gravitational radiation reaction of particles in curved spacetime", Phys. Rev. D, 56, 3381-3394, (1997). [gr-qc/9610053]. 6.5.3

[302] Raab, F.J., "The LIGO Project: Progress and Prospects", in Coccia, E., Pizzella, G., and Ronga, F., eds., Gravitational Wave Experiments, First Edoardo Amaldi Conference, Villa Tuscolana, Frascati, Rome, 14-17 June 1994, (World Scientific, Singapore; River Edge, NJ, 1995). 4.2.1, 4.3.1

[303] Raab, F.J. (for the LIGO Scientific Collaboration), "The status of laser interferometer gravitational-wave detectors", J. Phys.: Conf. Ser., 39, 25-31, (2006). [DOI]. 4.3 .1

[304] Randall, L., and Servant, G., "Gravitational waves from warped spacetime", J. High Energy Phys., 2007(05), 054, (2007). [DOI], [hep-ph/0607158]. 6.6.3

[305] Rees, M.J., "Gravitational waves from galactic centres?", Class. Quantum Grav., 14, 14111415, (1997). [DOI]. 3.4.5, 3.4.6

[306] Rees, M.J., and Meszaros, P., "Unsteady outflow models for cosmological gamma-ray bursts", Astrophys. J., 430, L93-L96, (1994). [astro-ph/9404038]. 6.1

[307] Regge, T., and Wheeler, J.A., "Stability of a Schwarzschild singularity", Phys. Rev., 108, 1063-1069, (1957). [DOI]. 6.4

[308] Reisenegger, A., and Bonacic, A.A., "Millisecond pulsars with r-modes as steady gravitational radiators", Phys. Rev. Lett., 91, 201103, (2003). [DOI], [astro-ph/0303375]. 3.3

[309] Rezzolla, L., Barausse, E., Dorband, E.N., Pollney, D., Reisswig, C., Seiler, J., and Husa, S., "On the final spin from the coalescence of two black holes", Phys. Rev. D, 78, 044002, (2007). [DOI], [arXiv:0712.3541]. 6.5.2

[310] Rezzolla, L., Diener, P., Dorband, E.N., Pollney, D., Reisswig, C., Schnetter, E., and Seiler, J., "The final spin from the coalescence of aligned-spin black hole binaries", Astrophys. J. Lett., 674, L29-L32, (2008). [DOI], [arXiv:0710.3345]. 11

[311] Rezzolla, L., Dorband, E.N., Reisswig, C., Diener, P., Pollney, D., Schnetter, E., and Szilágyi, B., "Spin Diagrams for Equal-Mass Black-Hole Binaries with Aligned Spins", Astrophys. J., 679, 1422-1426, (2007). [arXiv:0708.3999]. 6.5.2

[312] Richstone, D., "Supermassive Black Holes Then and Now", in Folkner, W.M., ed., Laser Interferometer Space Antenna (LISA), The Second International LISA Symposium on the Detection and Observation of Gravitational Waves in Space, Pasadena, California, July 1998, AIP Conference Proceedings, vol. 456, (American Institute of Physics, Woodbury, NY, 1998). [astro-ph/9810379]. 7.2.4 
[313] Riess, A.G., Filippenko, A.V., Challis, P., Clocchiatti, A., Diercks, A., Garnavich, P.M., Gilliland, R.L., Hogan, C.J., Jha, S., Kirshner, R.P., Leibundgut, B., Phillips, M.M., Reiss, D., Schmidt, B.P., Schommer, R.A., Smith, R.C., Spyromilio, J., Stubbs, C., Suntzeff, N.B., and Tonry, J., "Observational Evidence from Supernovae for an Accelerating Universe and a Cosmological Constant", Astrophys. J., 116, 1009-1038, (1998). [ADS], [astro-ph/9805201]. 1

[314] Robinson, C.A.K., Sathyaprakash, B.S., and Sengupta, A.S., "A geometric algorithm for efficient coincident detection of gravitational waves", Phys. Rev. D, 78, 062002, (2008). [arXiv:0804.4816]. 4.7.1

[315] Rover, C., Meyer, R., and Christensen, N., "Bayesian inference on compact binary inspiral gravitational radiation signals in interferometric data", Class. Quantum Grav., 23, 48954906, (2006). [gr-qc/0602067]. 6.5.4

[316] Rover, C., Meyer, R., and Christensen, N., "Coherent Bayesian inference on compact binary inspirals using a network of interferometric gravitational wave detectors", Phys. Rev. D, 75, 062004, (2007). [DOI], [gr-qc/0609131]. 6.5.4

[317] Ryan, F.D., "Gravitational waves from the inspiral of a compact object into a massive, axisymmetric body with arbitrary multipole moments", Phys. Rev. D, 52, 5707-5718, (1995). [DOI]. 3.4.6

[318] Ryan, F.D., "Accuracy of estimating the multipole moments of a massive body from the gravitational waves of a binary inspiral", Phys. Rev. D, 56, 1845-1855, (1997). [DOI]. 3.4 .6

[319] Sasaki, M., and Tagoshi, H., "Analytic Black Hole Perturbation Approach to Gravitational Radiation", Living Rev. Relativity, 6, lrr-2003-6, (2003). URL (cited on 03 September 2007): http://www. livingreviews.org/lrr-2003-6. 6.5.3, 6.6.2

[320] Sathyaprakash, B.S., "Problem of searching for spinning black hole binaries", in Dumarchez, J., and Trân Than Vân, J., eds., Gravitational Waves and Experimental Gravity, Proceedings of the XXXVIII Rencontres de Moriond, Les Arcs, France, March 22-29, 2003, (The Gioi Publishers, Hanoi, Vietnam, 2004). Related online version (cited on 17 December 2008):

http://moriond.in2p3.fr/J03/transparencies/6_friday/2_afternoon/ sathyaprakash.pdf. 3.4 .6

[321] Sathyaprakash, B.S., and Dhurandhar, S.V., "Choice of filters for the detection of gravitational waves from coalescing binaries", Phys. Rev. D, 44, 3819-3834, (1991). [DOI]. 5.1.3.1, 5.3 .1

[322] Sathyaprakash, B.S., and Schutz, B.F., "Templates for stellar mass black holes falling into supermassive black holes", Class. Quantum Grav., 20, S209-S218, (2003). [DOI], [grqc/0301049]. 3.4.6, 7.2

[323] Schmidt, M., "Spectrum of a Stellar Object Identified with the Radio Source 3C 286", Astrophys. J., 136, 684, (1962). [ADS]. 1

[324] Schneider, R., Ferrari, V., and Matarrese, S., "Stochastic backgrounds of gravitational waves from cosmological populations of astrophysical sources", Nucl. Phys. B (Proc. Suppl.), 80, C722, (2000). [astro-ph/9903470]. 7.3.1

[325] Schneider, R., Ferrari, V., Matarrese, S., and Portegies Zwart, S.F., "Gravitational waves from cosmological compact binaries", Mon. Not. R. Astron. Soc., 324, 797, (2001). [DOI], [astro-ph/0002055]. 8.2.2 
[326] Schreier, E., Levinson, R., Gursky, H., Kellogg, E., Tananbaum, H., and Giacconi, R., "Evidence for the Binary Nature of Centaurus X-3 from UHURU X-Ray Observations", Astrophys. J., 172, L79-L89, (1972). [DOI], [ADS]. 1

[327] Schutz, B.F., "Statistical formulation of gravitational radiation reaction", Phys. Rev. D, 22, 249-259, (1980). [DOI]. 6.5.3

[328] Schutz, B.F., "Gravitational Waves on the Back of an Envelope", Am. J. Phys., 52, 412-419, (1984). [DOI]. 2.4.2

[329] Schutz, B.F., "Determining the Hubble Constant from Gravitational Wave Observations", Nature, 323, 310-311, (1986). [DOI]. 3.4.2, 6.5.1, 8.3

[330] Schutz, B.F., ed., Gravitational Wave Data Analysis, Proceedings of the NATO Advanced Research Workshop held at Dyffryn House, St. Nichols, Cardiff, Wales, 6 - 9 July 1987, NATO ASI Series C, vol. 253, (Kluwer, Dordrecht; Boston, 1989). 5

[331] Schutz, B.F., "Data Processing, Analysis and Storage for Interferometric Antennas", in Blair, D.G., ed., The Detection of Gravitational Waves, pp. 406-452, (Cambridge University Press, Cambridge; New York, 1991). 5

[332] Schutz, B.F., A First Course in General Relativity, (Cambridge University Press, Cambridge; New York, 2009), 2nd edition. 2.6, 2.6

[333] Schutz, B.F., and Ricci, F., "Gravitational Waves, Sources and Detectors", in Ciufolini, I., Gorini, V., Moschella, U., and Frè, P., eds., Gravitational Waves, Lectures given at a school on 'Gravitational Waves in Astrophysics, Cosmology and String Theory', held in Como, Italy, 1999, Series in High Energy Physics, Cosmology and Gravitation, pp. 11-83, (Institute of Physics, Bristol, 2001). 7.1.1

[334] Schutz, B.F., and Tinto, M., "Antenna patterns of interferometric detectors of gravitational waves - I. Linearly polarized waves", Mon. Not. R. Astron. Soc., 224, 131-154, (1987). [ADS]. 4.2.1

[335] Searle, A.C., Sutton, P.J., Tinto, M., and Woan, G., "Robust Bayesian detection of unmodelled bursts", Class. Quantum Grav., 25, 114038, (2008). [DOI], [arXiv:0712.0196]. 5.2

[336] Shibata, M., and Uryū, K., "Merger of black hole-neutron star binaries in full general relativity", Class. Quantum Grav., 24, S125-S137, (2007). [DOI]. 7.3.2

[337] Sigurdsson, S., "Estimating the detectable rate of capture of stellar mass black holes by massive central black holes in normal galaxies", Class. Quantum Grav., 14, 1425-1429, (1997). [DOI], [astro-ph/9701079]. 3.4.6

[338] Sigurdsson, S., and Rees, M.J., "Capture of stellar-mass compact objects by massive black holes in galactic cusps", Mon. Not. R. Astron. Soc., 284, 318, (1996). [astro-ph/9608093]. 3.4 .6

[339] Sintes, A.M., and Krishnan, B., "Improved Hough search for gravitational wave pulsars", $J$. Phys.: Conf. Ser., 32, 206-211, (2006). [gr-qc/0601081]. 5.1.3.2

[340] Sivia, D.S., Data Analysis: A Bayesian Tutorial, (Oxford University Press, Oxford; New York, 1996). 5.3, 5.3.4

[341] Smak, J., "Light Variability of HZ 29", Acta Astron., 17, 255-270, (1967). [ADS]. 7.4 
[342] Smoot, G.F., Bennett, C.L., Kogut, A., Wright, E.L., Aymon, J., Boggess, N.W., Cheng, E.S., de Amici, G., Gulkis, S., Hauser, M.G., Hinshaw, G., Jackson, P.D., Janssen, M., Kaita, E., Kelsall, T., Keegstra, P., Lineweaver, C., Loewenstein, K., Lubin, P., Mather, J., Meyer, S.S., Moseley, S.H., Murdock, T., Rokke, L., Silverberg, R.F., Tenorio, L., and Weiss, R., "Structure in the COBE DMR First Year Maps", Astrophys. J. Lett., 396, L1-L5, (1992). [DOI], [ADS]. 8.1.4

[343] Spergel, D.N., Bean, R., Doré, O., Nolta, M.R., Bennett, C.L., Dunkley, J., Hinshaw, G., Jarosik, N., Komatsu, E., Page, L., Peiris, H.V., Verde, L., Halpern, M., Hill, R.S., Kogut, A., Limon, M., Meyer, S.S., Odegard, N., Tucker, G.S., Weiland, J.L., Wollack, E., and Wright, E.L., "Wilkinson Microwave Anisotropy Probe (WMAP) Three Year Results: Implications for Cosmology", Astrophys. J. Suppl. Ser., 170, 377-408, (2007). [astro-ph/0603449]. 8.2.1

[344] Stairs, I.H., "Testing General Relativity with Pulsar Timing", Living Rev. Relativity, 6, lrr2003-5, (2003). URL (cited on 03 September 2007):

http://www. livingreviews.org/lrr-2003-5. 3.4.3, 4.4.2

[345] Stappers, B.W., Kramer, M., Lyne, A.G., D’Amico, N., and Jessner, A., "The European Pulsar Timing Array", Chin. J. Astron. Astrophys. Suppl., 6, 298-303, (2006). [ADS]. 4.4.2

[346] Steigman, G., "Primordial Nucleosynthesis in the Precision Cosmology Era", Annu. Rev. Nucl. Part. Sci., 57, 463-491, (2007). [DOI], [arXiv:0712.1100]. 3.6, 8.2.1

[347] Stergioulas, N., and Friedman, J.L., "Nonaxisymmetric Neutral Modes in Rotating Relativistic Stars", Astrophys. J., 492, 301-322, (1998). [DOI], [gr-qc/9705056]. 7.3.4.1

[348] Stroeer, A., Gair, J.R., and Vecchio, A., "Automatic Bayesian inference for LISA data analysis strategies", in Merkowitz, S.M., and Livas, J.C., eds., Laser Interferometer Space Antenna, 6th International LISA Symposium, Greenbelt, Maryland, 19-23 June 2006, AIP Conference Proceedings, vol. 873, pp. 444-451, (American Institute of Physics, Melville, NY, 2006). [gr-qc/0609010]. 5.3.4

[349] Stroeer, A., and Vecchio, A., "The LISA verification binaries", Class. Quantum Grav., 23, S809-S818, (2006). [astro-ph/0605227]. 7.1

[350] Sumner, T.J., "Experimental Searches for Dark Matter", Living Rev. Relativity, 5, lrr-20024, (2002). URL (cited on 07 December 2004): http://www. livingreviews.org/lrr-2002-4. 8.2.1

[351] Sutherland, W., "Gravitational Microlensing - A Report on the MACHO Project", Rev. Mod. Phys., 71, 421-434, (1999). [astro-ph/9811185]. 7.2.2

[352] Syracuse University Gravitational Wave Group, "Numerical Injection Analysis Project Home Page", project homepage. URL (cited on 28 August 2008):

https://www.gravity.phy.syr.edu/dokuwiki/doku.php?id=ninja:home. 6.5.2

[353] Tagoshi, H., Mukhopadhyay, H., Dhurandhar, S., Sago, N., Takahashi, H., and Kanda, N., "Detecting gravitational waves from inspiraling binaries with a network of detectors: Coherent strategies by correlated detectors", Phys. Rev. D, 75, 087306, (2007). [DOI], [grqc/0702019]. 4.7.1

[354] Tagoshi, H., Shibata, M., Tanaka, T., and Sasaki, M., "Post-Newtonian expansion of gravitational waves from a particle in circular orbits around a rotating black hole: Up to $O\left(v^{8}\right)$ beyond the quadrupole formula", Phys. Rev. D, 54, 1439-1459, (1996). [gr-qc/9603028]. 6.5.3

Living Reviews in Relativity

http: //www . livingreviews . org/lrr-2009-2 
[355] Taylor, J.H., and Weisberg, J.M., "Further experimental tests of relativistic gravity using the binary pulsar PSR 1913+16", Astrophys. J., 345, 434-450, (1989). [ADS]. 3.4.3, 7

[356] Taylor Jr, J.H., "Nobel Lecture: Binary pulsars and relativistic gravity", Rev. Mod. Phys., 66, 711-719, (1994). [DOI]. Related online version (cited on 26 February 2009): http://nobelprize.org/nobel_prizes/physics/laureates/1993/taylor-lecture. html. 6.3

[357] Teukolsky, S.A., "Rotating black holes: Separable wave equations for gravitational and electromagnetic perturbations", Phys. Rev. Lett., 29, 1114-1118, (1972). [DOI]. 6.4

[358] Teukolsky, S.A., "Perturbations of a rotating black hole. I. Fundamental equations for gravitational electromagnetic and neutrino-field perturbations", Astrophys. J., 185, 635-647, (1973). [DOI]. 6.4

[359] Thorne, K.S., "Gravitational radiation", in Hawking, S.W., and Israel, W., eds., Three Hundred Years of Gravitation, pp. 330-458, (Cambridge University Press, Cambridge; New York, 1987). 3.6, 4.2.1, 4.7.3, $5,8.1 .1$

[360] Thorne, K.S., "Gravitational waves", in Kolb, E.W., and Peccei, R., eds., Particle and Nuclear Astrophysics and Cosmology in the Next Millennium, Proceedings of the 1994 Snowmass Summer Study, Snowmass, Colorado, June 29 - July 14, 1994, pp. 160-184, (World Scientific, Singapore; River Edge, NJ, 1995). 7.2

[361] Trias, M., and Sintes, A.M., "LISA observations of supermassive black holes: parameter estimation using full post-Newtonian inspiral waveforms", Phys. Rev. D, 77, 024030, (2008). [DOI], [arXiv:0707.4434]. 6.5.5, 15

[362] Tsubono, K., "300-m Laser Interferometer Gravitational Wave Detector (TAMA300) in Japan", in Coccia, E., Pizzella, G., and Ronga, F., eds., Gravitational Wave Experiments, First Edoardo Amaldi Conference, Villa Tuscolana, Frascati, Rome, 14-17 June 1994, pp. 112-114, (World Scientific, Singapore; River Edge, NJ, 1995). 4.3.1

[363] Tsubono, K. et al. (The TAMA Collaboration), "TAMA Project", in Tsubono, K., Fujimoto, M.-K., and Kuroda, K., eds., Gravitational Wave Detection, Proceedings of the TAMA International Workshop held at Saitama, Japan, November 12-14, 1996), Frontiers Science Series, vol. 20, pp. 183-191, (Universal Academy Press, Tokyo, 1997). [ADS]. 1

[364] Umstatter, R. et al., "Bayesian modeling of source confusion in LISA data", Phys. Rev. D, 72, 022001, (2005). [DOI], [gr-qc/0506055]. 6.5.4

[365] Ungarelli, C., and Vecchio, A., "High energy physics and the very early universe with LISA", Phys. Rev. D, 63, 064030, 1-14, (2001). [DOI]. 8.2.2

[366] Ungarelli, C., and Vecchio, A., "Studying the anisotropy of the gravitational wave stochastic background with LISA", Phys. Rev. D, 64, 121501, (2001). [astro-ph/0106538]. 8.2.2

[367] University of Rome 'La Sapienza', "Rome Gravitational Wave Group", project homepage. URL (cited on 08 November 2007):

http://www.roma1.infn.it/rog/. 4.1

[368] University of Western Australia, "AIGRC", project homepage. URL (cited on 08 November 2007): http://www.gravity.uwa.edu.au/. 4.3.1 
[369] University of Wisconsin at Milwaukee, "EinsteinATHome Project Home Page", project homepage. URL (cited on 08 November 2007):

http://einstein.phys.uwm.edu/. 5.1.3.2

[370] Ushomirsky, G., Cutler, C., and Bildsten, L., "Deformations of accreting neutron star crusts and gravitational wave emission", Mon. Not. R. Astron. Soc., 319, 902-932, (2000). [DOI], [astro-ph/0001136]. 3.3

[371] Vahlbruch, H., Mehmet, M., Chelkowski, S., Hage, B., Franzen, A., Lastzka, N., Gossler, S., Danzmann, K., and Schnabel, R., "Observation of Squeezed Light with 10-dB QuantumNoise Reduction", Phys. Rev. Lett., 100, 033602, (2008). [DOI], [arXiv:0706.1431]. 4

[372] Vallisneri, M., "Use and abuse of the Fisher information matrix in the assessment of gravitational-wave parameter-estimation prospects", Phys. Rev. D, 77, 042001, (2008). [DOI], [gr-qc/0703086]. 6.5.4

[373] Valtonen, M.J., Lehto, H.J., Nilsson, K., Heidt, J., Takalo, L.O., Sillanpää, A., Villforth, C., Kidger, M., Poyner, G., Pursimo, T., Zola, S., Wu, J.-H., Zhou, X., Sadakane, K., Drozdz, M., Koziel, D., Marchev, D., Ogloza, W., Porowski, C., Siwak, M., Stachowski, G., Winiarski, M., Hentunen, V.-P., Nissinen, M., Liakos, A., and Dogru, S., "A massive binary black-hole system in OJ 287 and a test of general relativity", Nature, 452, 851-853, (2008). [DOI]. 7.2.4

[374] Van Den Broeck, C., and Sengupta, A.S., "Binary black hole spectroscopy", Class. Quantum Grav., 24, 1089-1114, (2007). [DOI], [gr-qc/0610126]. 6.5.5, 6.5.5, 8.3

[375] Van Den Broeck, C., and Sengupta, A.S., "Phenomenology of amplitude-corrected postNewtonian gravitational waveforms for compact binary inspiral. I. Signal-to-noise ratios", Class. Quantum Grav., 24, 155-176, (2007). [gr-qc/0607092]. 6.5.5

[376] van der Klis, M., "Kilohertz quasi-periodic oscillations in low-mass X-ray binaries", in Buccheri, R., van Paradijs, J., and Alpar, M.A., eds., The Many Faces of Neutron Stars, Proceedings of the NATO Advanced Study Institute, Lipary, Italy, September 30 - October 11, 1996, NATO ASI Series, vol. 515, pp. 337-368, (Kluwer Academic Publishers, Dordrecht, 1998). 7.3 .5

[377] Veitch, J., and Vecchio, A., "Assigning confidence to inspiral gravitational wave candidates with Bayesian model selection", Class. Quantum Grav., 25, 184010, (2008). [DOI], [arXiv:0807.4483]. 5.3.4

[378] Veitch, J., and Vecchio, A., "A Bayesian approach to the follow-up of candidate gravitational wave signals", Phys. Rev. D, 78, 022001, (2008). [DOI], [arXiv:0801.4313]. 5.3.4

[379] Vilenkin, A., and Shellard, E.P.S., Cosmic Strings and Other Topological Defects, Cambridge Monographs on Mathematical Physics, (Cambridge University Press, Cambridge, 1994). 8.2.1

[380] VIRGO Project, "VIRGO Project Home Page", project homepage. URL (cited on 08 November 2007): http://wwwcascina.virgo.infn.it/. 4.3.1

[381] Vishveshwara, C.V., "Scattering of gravitational radiation by a Schwarzschild black-hole", Nature, 227, 936-938, (1970). [DOI]. 3.5, 6.4

[382] Vishveshwara, C.V., "Stability of the Schwarzschild metric", Phys. Rev. D, 1, 2870-2879, (1970). 6.4 
[383] Wagoner, R.V., "Gravitational radiation from accreting neutron stars", Astrophys. J., 278, 345-348, (1984). [DOI], [ADS]. 3.3

[384] Watson, A.A., "Observations of ultra-high energy cosmic rays", J. Phys.: Conf. Ser., 39, 365-371, (2006). [astro-ph/0511800]. 7.4

[385] Watts, A., Krishnan, B., Bildsten, L., and Schutz, B.F., "Detecting gravitational wave emission from the known accreting neutron stars", Mon. Not. R. Astron. Soc., accepted, (2008). [arXiv:0803.4097]. 7, 7.3.5, 7.4

[386] Watts, A.L., and Strohmayer, T.E., "High frequency oscillations during magnetar flares", Astrophys. Space Sci., 308, 625-629, (2007). [astro-ph/0608476]. 7.3.3

[387] Weber, J., "Gravitational radiation", Phys. Rev. Lett., 18, 498-501, (1967). [DOI]. 4

[388] Weisberg, J.M., and Taylor, J.H., "The Relativistic Binary Pulsar B1913+16: Thirty Years of Observations and Analysis", in Rasio, F.A., and Stairs, I.H., eds., Binary Radio Pulsars, Proceedings of a meeting held at the Aspen Center for Physics, USA, 12 -16 January 2004, ASP Conference Series, vol. 328, pp. 25-32, (Astronomical Society of the Pacific, San Francisco, 2005). [astro-ph/0407149]. 1.2, 3.4.3

[389] Wen, L., and Gair, J.R, "Detecting extreme mass ratio inspirals with LISA using timefrequency methods", Class. Quantum Grav., 22, S445-S452, (2005). [DOI], [gr-qc/0502100]. 5.2

[390] Wen, L., and Schutz, B.F., "Coherent network detection of gravitational waves: the redundancy veto", Class. Quantum Grav., 22, S1321-S1336, (2005). [gr-qc/0508042]. 4.7.2

[391] Whelan, J.T., Daw, E., Heng, I.S., McHugh, M.P., and Lazzarini, A., "Phenomenological template family for black-hole coalescence waveforms", Class. Quantum Grav., 20, S689, (2003). [gr-qc/0308045]. 8.1.2

[392] Will, C.M., Theory and Experiment in Gravitational Physics, (Cambridge University Press, Cambridge; New York, 1993), 2nd edition. 5

[393] Will, C.M., "Bounding the mass of the graviton using gravitional-wave observations of inspiralling compact binaries", Phys. Rev. D, 57, 2061-2068, (1998). [DOI], [gr-qc/9709011]. 6.1, 6.6 .1

[394] Will, C.M., "The Confrontation between General Relativity and Experiment", Living Rev. Relativity, 9, lrr-2006-3, (2006). URL (cited on 03 September 2007): http://www.livingreviews.org/lrr-2006-3. 3.4.3, 6.2, 6.3

[395] Willke, B., Ajith, P., Allen, B., Aufmuth, P., and Aulbert, C. et al., "The GEO-HF project", Class. Quantum Grav., 23, S207-S214, (2006). [DOI]. 4, 4.3.1

[396] Willke, B. (for the LIGO Scientific Collaboration), "GEO600: status and plans", Class. Quantum Grav., 24, S389-S397, (2007). 4.3.1

[397] Woosley, S.E., "Gamma-ray bursts from stellar mass accretion disks around black holes", Astrophys. J., 405, 273-277, (1993). [DOI], [ADS]. 3.2

[398] Zerilli, F.J., "Gravitational Field of a Particle Falling in a Schwarzschild Geometry Analyzed in Tensor Harmonics", Phys. Rev. D, 2, 2141-2160, (1970). [DOI]. 6.4 